\title{
DIRICHLET PROBLEMS FOR A NONSTATIONARY LINEARIZED SYSTEM OF NAVIER-STOKES EQUATIONS IN NON-CYLINDRICAL DOMAINS*
}

\author{
Steve HofmanN ${ }^{\dagger}$ AND KaJ Nyström ${ }^{\ddagger}$
}

Abstract. In this paper we consider the solvability of $L^{2}$-boundary value problems for a nonstationary linearized system of Navier-Stokes in the time-varying cylinders of Hofmann and Lewis [19], i.e., we consider the problem

$$
\begin{gathered}
\frac{\partial \vec{u}}{\partial t}=\Delta \vec{u}-\nabla p \text { in } \Omega \\
\operatorname{div} \vec{u}=0 \text { in } \Omega
\end{gathered}
$$

in a class of time-varying, infinite cylinders

$$
\Omega=\left\{\left(x_{0}, x, t\right) \in \mathbb{R} \times \mathbb{R}^{n-1} \times \mathbb{R}: x_{0}>A(x, t)\right\},
$$

with Dirichlet and Neumann type boundary conditions.

0. Introduction. Let $D \subset \mathbb{R}^{n}$ be an open set. The Navier-Stokes equations is a $n+1$ by $n+1$ system of equations for the unknowns $u_{1}(x, t), \ldots, u_{n}(x, t)$ representing a velocity vector and $p(x, t)$ representing specific pressure. The variables $(x, t) \in D \times \mathbb{R}_{+}^{1}$ represent position and time. The equations are,

$$
\text { (1) } \frac{\partial \vec{u}}{\partial t}-v \Delta \vec{u}+\vec{u} \cdot \nabla \vec{u}+\nabla p=\vec{F},
$$

(2) $\operatorname{div} \vec{u}=0$.

The coefficient $v>0$ is called the kinematic viscosity coefficient and $\vec{F}(x, t)$ is the specific body force. The equations are supplemented with boundary conditions and in the initial boundary value problem $\vec{u}(x, t)$ is assumed to be known for $t=0$. For the fundamentals on the Navier-Stokes equation we refer the reader to the book of Constantin-Foias [6]. It is of interest to study linearized versions of the equation in (1). In this paper we consider a nonstationary linearized version of the Navier-Stokes equations and we are interested in solvability of this system using the relevant single and double layer potentials. To be precise we consider,

$$
\begin{gathered}
\frac{\partial \vec{u}}{\partial t}=\Delta \vec{u}-\nabla p \text { in } \Omega \\
\operatorname{div} \vec{u}=0 \text { in } \Omega,
\end{gathered}
$$

with $L^{2}$-boundary data and the main novelty is that we consider this problem in a class of nonsmooth time-varying domains $\Omega$. It is a well known principle in the study of nonstationary nonlinear partial differential equations that a careful study of an associated linear problem quite often yields information for the nonlinear problem at least for some time interval $\left[0, T_{0}\right)$. This is also true in the case of Navier-Stokes

\footnotetext{
* Received September 12, 2000; accepted for publication February 23, 2001.

$\dagger$ Department of Mathematics, University of Missouri, Columbia, MO 65211-0001, USA (hofmann@msindy2.cs.missouri.edu).

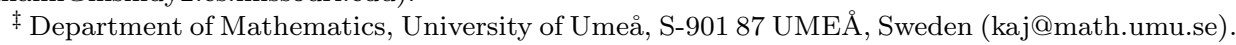


equations, see [12] where the Navier-Stokes equations are studied in smooth timeindependent cylinders and where the solvability of the system in $(*)$ plays an important role. The stationary version of the system in $(*)$ is often referred to as the Stokes system. In recent years solvability and regularity results for the Stokes system have been established in as non smooth domains as Lipschitz, see [10][14][31]. These results have also proven useful in the study of long-time behaviour of solutions to initial boundary value problems for the Navier-Stokes equations in infinite time-independent Lipschitz cylinders, see [11][3].

The nonstationary linearized system of Navier-Stokes, i.e. the system in $(*)$ was studied by Shen [32] in time-independent Lipschitz cylinders and this paper generalizes to a certain extent the results of Shen to the setting of nonsmooth time-dependent cylinders. This should not be misunderstood in the sense that this is not a straightforward generalization.

Before proceeding we would shortly like to describe some of the development of partial differential equations in as nonsmooth domains as Lipschitz in order to put our paper in perspective. In fact, in the last 20 years there has been a considerable amount of activity in the study of boundary value problems in Lipschitz domains with $L^{p}$ boundary data. For the Laplace operator the Dirichlet and Neumann problems are well understood (see [24][30]). The initial Dirichlet and Neumann problems for the heat operator in nonsmooth time-independent cylinders have also been analyzed in depth (see [1][2]). Also, as mentioned above in case of the Stokes system, the theory of systems has reached a considerable level of maturity. In [10] and [14] $L^{2}$ solvability for boundary value problems in Lipschitz domains for the Stokes system and the systems of elastostatics are considered. Parabolic versions of these results are proved in [32]. There are furthermore several other important contributions to the study of linear systems of partial differential equations on domains as nonsmooth as Lipschitz, see e.g. [9][17].

In [19] Hofmann and Lewis were able to find the optimal condition on a timevarying cylinder in order to garantee the solvability of $L^{2}$-boundary value problems for the heat operator in the time-varying cylinder. In [20], [21] these problems are studing in the setting of $L^{p}$-boundary data. This is an impressive achievement and concludes a development to which several researchers have contributed (see [18][19][22][25][26]). In [27] Nyström studied the solvability of $L^{2}$-boundary value problems for the so called parabolic Lamé system in the time-varying cylinders of Hofmann and Lewis.

In order to formulate the results of this paper we have to introduce some notation and give a description of our time-varying cylinders.

Our geometric set up is graph domains of the form

$$
\Omega=\left\{\left(x_{0}, x, t\right) \in \mathbb{R} \times \mathbb{R}^{n-1} \times \mathbb{R}: x_{0}>A(x, t)\right\}
$$

where $n \geq 2$. We have to impose restrictions on $A(x, t)$ and in order to do so we need to introduce some parabolic concepts.

Let $z=(x, t) \in \mathbb{R}^{n-1} \times \mathbb{R}$ and we let $\|z\|$ denote the parabolic norm of $z$. Note that $\left\|\left(\delta x, \delta^{2} t\right)\right\|=\delta\|(x, t)\|$. Recall that parabolic $B M O$ is the space of locally integrable functions modulo constants satisfying

$$
\|b\|_{*}:=\sup _{B} \frac{1}{|B|} \int_{B}\left|b(z)-m_{B} b\right| d z<\infty
$$


where $z=(x, t)$ and $B$ denotes the parabolic ball

$$
\begin{gathered}
B=B_{r}\left(z_{0}\right)=\left\{z \in \mathbb{R}^{n}:\left\|z-z_{0}\right\|<r\right\} \\
m_{B} b=\frac{1}{|B|} \int_{B} b(z) d z \\
|B|=\left|B_{r}\left(z_{0}\right)\right|=c(n) r^{d} \quad \text { where } \quad d=n+1 .
\end{gathered}
$$

Let $\wedge, \vee$ be the Fourier and the inverse Fourier transform on $\mathbb{R}^{n}$, and let $\xi, \tau$ denote the phase variables. Following Fabes-Riviere[15] we define a parabolic half-order time derivative by

$$
\mathbb{D}_{n} A(x, t):=\left(\frac{\tau}{\|(\xi, \tau)\|} \hat{A}(\xi, \tau)\right)^{\vee}(x, t) .
$$

Define

$$
\|A\|_{\mathrm{comm}}=\left\|\nabla_{x} A\right\|_{\infty}+\left\|\mathbb{D}_{n} A\right\|_{*}
$$

where

$$
\nabla_{x}=\left(\frac{\partial}{\partial x_{1}}, \ldots, \frac{\partial}{\partial x_{n-1}}\right)
$$

and $\left\|\nabla_{x} A\right\|_{\infty}:=\sup _{t}\left\|\nabla_{x} A(\cdot, t)\right\|_{\infty}$. The basic geometric restriction we impose on our domains is that

$$
\|A\|_{\text {comm }}=\left\|\nabla_{x} A\right\|_{\infty}+\left\|\mathbb{D}_{n} A\right\|_{*}<\infty .
$$

The main reasons why we consider domains defined by a function $A(x, t)$ satisfying such a smoothness condition are the results of S. Hofmann[18] on singular integrals on these domains and Hofmann-Lewis[19] results on boundary value problems for the heat equation in these domains. In fact, as explained in [18, p.213] the notation $\|A\|_{\text {comm }}$ reflects the fact that this quantity is equivalent to the operator norm of the first commutator $[\sqrt{\Delta-\partial / \partial t}, A]$. Since this commutator is the parabolic analogue of the first Calderon commutator, the present condition is, at least from the point of singular integrals the appropriate parabolic analogue of the Lipschitz domains which have been considered in the elliptic theory. In particular, one can not expect to relax the condition on $A$ to $\operatorname{Lip}_{1 / 2}$ in the time variable [22]. We define a surface measure on $\partial \Omega$ as $d \sigma_{t} d t$, where $d \sigma_{t}$ is the naturally defined surface measure on the Lipschitz graph $\partial \Omega_{t}, \Omega_{t}=\left\{\left(x_{0}, x, t\right) \in \mathbb{R} \times \mathbb{R}^{n-1} \times\{t\} ; x_{0}>A(x, t)\right\}$. The unit outer normal to $\Omega_{t}$ is denoted by $N_{t}=\left(N_{t}^{0}, \ldots, N_{t}^{n-1}\right)$.

We define $L^{p}(\partial \Omega)$ to be $L^{p}$-spaces w.r.t. the measure $d \sigma_{t} d t$ and following Fabes and Jodeit[13] we define a parabolic Sobolev space in the following way. Let $\pi: \partial \Omega \rightarrow \mathbb{R}^{n}$ be the projection $\pi(A(x, t), x, t)=(x, t)$ and set $\tilde{f}=f \circ \pi^{-1} \cdot L_{1,1 / 2}^{p}(\partial \Omega)$ consists of equivalence classes of functions $f$ with distributional derivatives in $x$ satisfying $\|f\|_{L_{1,1 / 2}^{p}(\partial \Omega)}<\infty$, where

$$
\|f\|_{L_{1,1 / 2}^{p}(\partial \Omega)}:=\|\tilde{f}\|_{L_{1,1 / 2}^{p}\left(\mathbb{R}^{n}\right)}:=\|\mathbb{D} \tilde{f}\|_{p}
$$

Here,

$$
(\mathbb{D} \tilde{f})^{\wedge}(\xi, \tau):=\|(\xi, \tau)\| \tilde{\tilde{f}}(\xi, \tau)
$$


i.e.

$$
\tilde{f}=\mathbb{D}^{-1} \phi, \quad \phi \in L^{p}\left(\mathbb{R}^{n}\right),
$$

where $\mathbb{D}^{-1}$ is a parabolic Riesz potential. If $p=2$ we have, by applying Plancherel's theorem, that

$$
\|\mathbb{D} \tilde{f}\|_{2} \approx\left\|D_{1 / 2}^{t} \tilde{f}\right\|_{2}+\left\|\nabla_{x} \tilde{f}\right\|_{2} .
$$

Here $D_{1 / 2}^{t}$ denotes the one-dimensional one half fractional derivative of $f$ in the time variable.

Recall that for $0<\alpha \leq 2$ and $g \in C_{0}^{\infty}(\mathbb{R})$ the fractional differentiation operators $D_{\alpha}$ are defined by

$$
\left(D_{\alpha} g\right)^{\wedge}(\tau):=|\tau|^{\alpha} \hat{g}(\tau) .
$$

It is well-known that if $0<\alpha<1$ then

$$
D_{\alpha} g(s)=c \int_{\mathbb{R}} \frac{g(s)-g(\tau)}{|s-\tau|^{1+\alpha}} d \tau,
$$

whenever $s \in \mathbb{R}$. $I_{\alpha}=c D_{\alpha}^{-1}$, where $I_{\alpha}(s)=|s|^{\alpha-1}$ for $s \in \mathbb{R}$ is the one-dimensional Riesz transform of order $\alpha$ and $c$ is a universal constant. If $h \in C_{0}^{\infty}\left(\mathbb{R}^{n}\right)$ then by $D_{\alpha}^{t} h: \mathbb{R}^{n} \rightarrow \mathbb{R}$ we will mean $D_{\alpha} h(x, \cdot)$ defined a.e. for each $x \in \mathbb{R}^{n-1}$. In [19] it is proved that

$$
\|A\|_{\text {comm }}:=\left\|\nabla_{x} A\right\|_{\infty}+\left\|\mathbb{D}_{n} A\right\|_{*} \approx\left\|\nabla_{x} A\right\|_{\infty}+\left\|D_{1 / 2}^{t} A\right\|_{*}
$$

and that given $\varepsilon>0,0<\varepsilon<1$ and $\gamma, 0<\gamma<\infty$ there exists $\delta=\delta(\varepsilon, \gamma)>0$ s.t. if $\left\|\nabla_{x} A\right\|_{\infty} \leq \gamma<\infty$ then

$$
\min \left\{\left\|D_{1 / 2}^{t} A\right\|_{*},\left\|\mathbb{D}_{n} A\right\|_{*}\right\} \leq \delta \Rightarrow \max \left\{\left\|D_{1 / 2}^{t} A\right\|_{*},\left\|\mathbb{D}_{n} A\right\|_{*}\right\} \leq \varepsilon .
$$

I.e. the smallness of $\left\|\mathbb{D}_{n} A\right\|_{*}$ could equivalently be stated as a smallness condition on $\left\|D_{1 / 2}^{t} A\right\|_{*}$.

One may also prove that $\|A\|_{\text {comm }} \leq \beta<\infty$ implies that $A(x, t)$ is parabolically Lipschitz in the following sense,

$$
|A(x, t)-A(y, s)| \leq \beta\left(|x-y|+|t-s|^{1 / 2}\right) \quad x, y \in \mathbb{R}^{n} \quad t, s \in \mathbb{R} .
$$

As mentioned above we are interested in solvability of the system in (*) using the relevant single and double layer potentials. As our cylinders are non smooth we can not expect to use the Fredholm theory of compact operators to prove invertibility of the boundary operators that naturally appear. Instead we follow the by now "standard" approach on domains of Lipschitz type as laid out in Verchota[30]. I.e using the relevant Rellich-type equalities we try to relate the $L^{2}$-norm of conormal derivatives of our singular layer potential to a $L^{2}$-regularity norm on the boundary. In this paper we do carry out all the necessary steps in order to prove the relevant Rellich-type inequalities for the system in $(*)$ on smooth approximating domains $\Omega^{\epsilon}$ defined below. The constants of our inequalities are independent of $\epsilon$. As the reader will notice this is a nontrivial task in the case of our nonsmooth time-dependent domains. Equipped with these inequalities we then approach questions of invertibility and existence for the Dirichlet-, Neumann-, and regularity-type problems on the approximating domains $\Omega^{\epsilon}$ and on the limiting domain $\Omega=\Omega^{0}$. 
Let $P(z) \in C_{0}^{\infty}\left(\mathbb{R}^{n}\right)$. We furthermore assume that $P(z)$ is a non-negative function and that $\int_{\mathbb{R}^{n}} P(z) d z=1$. I.e. we assume that $P(z)$ is a parabolic approximate identity. Let $d=n+1$ and define

$$
P_{\lambda}(z)=\lambda^{-d} P\left(\lambda^{-\alpha} z\right)=\lambda^{-d} P\left(\frac{x}{\lambda}, \frac{t}{\lambda^{2}}\right) .
$$

For a locally integrabel function $f$ we let $P_{\lambda} f(z)$ be the naturally defined convolution operator. The "parabolic" lifting $\rho(\lambda, x, t)$ from $\mathbb{R}_{+}^{n+1}=\mathbb{R}_{+} \times \mathbb{R}^{n-1} \times \mathbb{R}$ onto $\Omega=$ $\left\{\left(x_{0}, x, t\right) \in \mathbb{R} \times \mathbb{R}^{n-1} \times \mathbb{R}: x_{0}>A(x, t)\right\}$ defined in the following way,

$$
\rho(\lambda, x, t)=\left(\lambda+P_{\gamma \lambda} A(x, t), x, t\right) \quad \rho(0, x, t)=(A(x, t), x, t),
$$

plays, as described in Section 1, an important role in our paper. Let for $\epsilon>0$

$$
\begin{gathered}
\Omega^{\epsilon}=\left\{\left(x_{0}, x, t\right) \in \mathbb{R} \times \mathbb{R}^{n-1} \times \mathbb{R}: x_{0}>\epsilon+P_{\gamma \epsilon} A(x, t)\right\} \\
L_{N}^{2}\left(\partial \Omega^{\epsilon}\right)=\left\{\vec{f} \in L^{2}\left(\partial \Omega^{\epsilon}\right): \int_{\partial \Omega_{t}^{\epsilon}}\left\langle\vec{f}, N_{t}\right\rangle d \sigma_{t}^{\epsilon}=0 \quad \text { for almost all t }\right\}
\end{gathered}
$$

We define a regularity space on $\partial \Omega^{\epsilon}$ in the following way,

$$
R\left(\partial \Omega^{\epsilon}\right)=\left\{\vec{g} \in L_{1,1 / 2}^{2}\left(\partial \Omega^{\epsilon}\right) \cap L_{N}^{2}\left(\partial \Omega^{\epsilon}\right),\left\langle\frac{\partial \vec{g}}{\partial t}, N_{t}^{\epsilon}\right\rangle \in L^{2}\left(-\infty, \infty,\left[L_{1}^{2}\left(\partial \Omega_{t}^{\epsilon}\right)\right]^{*}\right)\right\},
$$

where $\left[L_{1}^{2}\left(\partial \Omega_{t}^{\epsilon}\right)\right]^{*}$ is the dual space of $L_{1}^{2}\left(\partial \Omega_{t}^{\epsilon}\right)$. For each $t, L_{1}^{2}\left(\partial \Omega_{t}^{\epsilon}\right)$ consists of functions with distributional derivatives in $x$ satisfying $\|f\|_{L_{1}^{2}\left(\partial \Omega_{t}^{\epsilon}\right)}<\infty$, where $\|f\|_{L_{1}^{2}\left(\partial \Omega_{t}^{\epsilon}\right)}=\left\|\nabla_{x} \tilde{f}\right\|_{2}$ and $\tilde{f}=f \circ \pi^{-1}, \pi(A(x, t), x, t)=(x, t)$. The norm of an element $\vec{g} \in R\left(\partial \Omega^{\epsilon}\right)$ is defined as

$$
\|\vec{g}\|_{L_{1,1 / 2}^{2}\left(\partial \Omega^{\epsilon}\right)}+\left[\int_{-\infty}^{\infty}\left\|\left\langle\frac{\partial \vec{g}}{\partial t}, N_{t}^{\epsilon}\right\rangle\right\|_{\left[L_{1}^{2}\left(\partial \Omega_{t}^{\epsilon}\right)\right]^{*}}^{2} d t\right]^{1 / 2}
$$

For more details on this space we refer to Section 9. In Section 8 the relevant Rellichtype inequalities containing the regularity norm of $R\left(\partial \Omega^{\epsilon}\right)$ are proved on $\partial \Omega^{\epsilon}$ with constants independent of $\epsilon$ provided $\|A\|_{\text {comm }} \leq \beta<\infty,\left\|D_{1 / 2}^{t} A\right\|_{*} \leq \epsilon_{0}<\infty$ with $\epsilon_{0}=\epsilon_{0}\left(\left\|\nabla_{x} A\right\|_{\infty}\right)$ small enough. These inequalities enable us to prove solvability for the regularity problem in $\partial \Omega^{\epsilon}$. One may then hope to use this result to study a regularity problem in the nonsmooth cylinder $\Omega=\Omega^{0}=\left\{\left(x_{0}, x, t\right) \in \mathbb{R} \times \mathbb{R}^{n-1} \times \mathbb{R}\right.$ : $\left.x_{0}>A(x, t)\right\}$ by some approximation argument. To us this seems to be a delicate and subtle point and we have decided to treat the issue separately in an other paper. One resonable thing to do in order to handle the regularity problem is to make use of the ordinary Dirichlet problem and in particular the double layer potential to extend the data into the domain in a way adapted to the problem and then hope that the restriction to $\partial \Omega^{\epsilon}$ of the extension belongs to $R\left(\partial \Omega^{\epsilon}\right)$ and that the $R\left(\partial \Omega^{\epsilon}\right)$-norm of the restriction is bounded with a constant independent of $\epsilon$. In the case of the system in $(*)$ the relevant double layer potential of $\vec{f} \in L^{2}(\partial \Omega)$ is defined in the following way,

$$
\vec{v}(X, t)=D \vec{f}(X, t):=\int_{-\infty}^{t} \int_{\partial \Omega_{s}} \frac{\partial \Gamma}{\partial N_{s}(Q)}(X-Q, t-s) \vec{f}(Q, s) d \sigma_{s}(Q) d s
$$




$$
-\int_{\partial \Omega_{t}} \frac{Q-X}{w_{n}|X-Q|^{n}}\left\langle\vec{f}(Q, t), N_{t}(Q)\right\rangle d \sigma_{t}(Q) .
$$

For more details we refer the reader to Section 2. What we want to point out is that formally $D \vec{f}$ solves the equation in $(*)$ with associated pressure given by

$$
\frac{\partial}{\partial t} \int_{\partial \Omega_{t}} \frac{1}{w_{n}|X-Q|^{n-2}}\left\langle\vec{f}(Q, t), N_{t}(Q)\right\rangle d \sigma_{t}(Q),
$$

i.e. the double layer is not a classical solution and weak solutions has to be defined. The fact that one has to resort to weak solutions complicate matters both in the Dirichlet problem and in the regularity problem in the nonsmooth time-dependent cylinder and we have decided (as mentioned above) to return to the regularity problem in an other paper. We feel that leaving that issue to a separate paper serves to emphasize that our results are non trivial generalizations of the results of Shen [32].

We are now ready to formulate our theorems. Apart from these theorem many other results are proved in the bulk of the paper. Let in the following the symbols $\tilde{N}_{*}, \tilde{N}_{*, e}$ refer to non tangential maximal function operators defined in the bulk of the paper. The goal in case of the Dirichlet problem is to prove the following theorem, Theorem A. For the definition of weak solutions we refer to Section 9.

Theorem A. (The Dirichlet problem) Let $\Omega=\left\{\left(x_{0}, x, t\right) \in \mathbb{R} \times \mathbb{R}^{n-1} \times \mathbb{R}: x_{0}>\right.$ $A(x, t)\}$ where $\|A\|_{\text {comm }} \leq \beta<\infty$ and $\left\|D_{1 / 2}^{t} A\right\|_{*} \leq \epsilon_{0}<\infty$. If $\epsilon_{0}=\epsilon_{0}\left(\left\|\nabla_{x} A\right\|_{\infty}\right)$ is small enough then the following is true. Given $\vec{f} \in L_{N}^{2}(\partial \Omega)$ there exists a unique weak solution $\vec{u}$ solving,

$$
\begin{aligned}
& \frac{\partial \vec{u}}{\partial t}=\Delta \vec{u}-\nabla p \quad \text { in } \quad \Omega, \\
& \text { divu }=0 \text { in } \Omega \\
& \vec{u}=\vec{f} \quad \text { a.e on } \quad \partial \Omega, \\
& \left\|\tilde{N}_{*, e}(\vec{u})\right\|_{2}<\infty .
\end{aligned}
$$

There furthermore exists $\vec{g} \in L^{2}(\partial \Omega)$ such that $\vec{u}$ can be represented as a double layer potential, $\vec{u}=D \vec{g}$, and

$$
\left\|\tilde{N}_{*, e}(D \vec{g})\right\|_{2} \leq C_{\beta}\|\vec{f}\|_{2}
$$

In the following two theorems $p$ is unique modulo a function just depending on time and $H D_{1 / 2}^{t}$ is the composition of the half time derivative operator described above and the Hilbert transform in $t$. The following Neumann problem is treated.

Theorem B. (Neumann problem) Let $\Omega=\left\{\left(x_{0}, x, t\right) \in \mathbb{R} \times \mathbb{R}^{n-1} \times \mathbb{R}: x_{0}>\right.$ $A(x, t)\}$ where $\|A\|_{\text {comm }} \leq \beta<\infty$ and $\left\|D_{1 / 2}^{t} A\right\|_{*} \leq \epsilon_{0}<\infty$. If $\epsilon_{0}=\epsilon_{0}\left(\left\|\nabla_{x} A\right\|_{\infty}\right)$ is small enough then the following is true. If $\vec{f} \in L^{2}(\partial \Omega)$ then there exists a unique solution $(\vec{u}, p)$ to the problem,

$$
\begin{aligned}
& \frac{\partial \vec{u}}{\partial t}=\Delta \vec{u}-\nabla p \text { in } \Omega \\
& \text { div } \vec{u}=0 \text { in } \Omega \\
& \frac{\partial \vec{u}}{\partial \nu}=(\nabla \vec{u}) N-p N=\vec{f} \text { on } \partial \Omega
\end{aligned}
$$




$$
\left\|\tilde{N}_{*}(\vec{u})\right\|_{2}+\left\|\tilde{N}_{*}(\nabla \vec{u})\right\|_{2}+\left\|\tilde{N}_{*, e}(p)\right\|_{2}<\infty .
$$

Furthermore there exists $\vec{g} \in L^{2}(\partial \Omega)$ such that $\vec{u}$ can be represented as a single layer potential, $\vec{u}=S \vec{g}$, and

$$
\left\|\tilde{N}_{*}(\vec{u})\right\|_{2}+\left\|\tilde{N}_{*}(\nabla \vec{u})\right\|_{2}+\left\|\tilde{N}_{*}\left(H D_{1 / 2}^{t} \vec{u}\right)\right\|_{2}+\left\|\tilde{N}_{*, e}(p)\right\|_{2} \leq C\|\vec{f}\|_{2} .
$$

We have the following result on the regularity problem.

Theorem C. (Dirichlet regularity problem on the smooth approximating domains) Let $\Omega^{\epsilon}=\left\{\left(x_{0}, x, t\right) \in \mathbb{R} \times \mathbb{R}^{n-1} \times \mathbb{R}: x_{0}>\epsilon+P_{\gamma \epsilon} A(x, t)\right\}$ where $\|A\|_{\text {comm }} \leq$ $\beta<\infty$ and $\left\|D_{1 / 2}^{t} A\right\|_{*} \leq \epsilon_{0}<\infty$. If $\epsilon_{0}=\epsilon_{0}\left(\left\|\nabla_{x} A\right\|_{\infty}\right)$ is small enough then the following is true. If $\vec{f} \in R\left(\partial \Omega^{\epsilon}\right)$ then there exists a unique solution $(\vec{u}, p)$ to the problem,

$$
\begin{aligned}
& \frac{\partial \vec{u}}{\partial t}=\Delta \vec{u}-\nabla p \text { in } \Omega^{\epsilon} \\
& \operatorname{div} \vec{u}=0 \text { in } \Omega^{\epsilon} \\
& \vec{u}=\vec{f} \text { on } \partial \Omega^{\epsilon} \\
& \left\|\tilde{N}_{*}(\vec{u})\right\|_{2}+\left\|\tilde{N}_{*}(\nabla \vec{u})\right\|_{2}+\left\|\tilde{N}_{*, e}(p)\right\|_{2}<\infty .
\end{aligned}
$$

Furthermore there exists $\vec{g} \in L^{2}\left(\partial \Omega^{\epsilon}\right)$ such that $\vec{u}$ can be represented as a single layer potential, $\vec{u}=S \vec{g}$, and a constant $C$ independent of $\epsilon$ such that

$$
\left\|\tilde{N}_{*}(\vec{u})\right\|_{2}+\left\|\tilde{N}_{*}(\nabla \vec{u})\right\|_{2}+\left\|\tilde{N}_{*}\left(H D_{1 / 2}^{t} \vec{u}\right)\right\|_{2}+\left\|\tilde{N}_{*, e}(p)\right\|_{2} \leq C\|\vec{f}\|_{R\left(\partial \Omega^{\epsilon}\right)} .
$$

The paper is divided into the following sections,

1. Carleson measures and maximal functions.

2. Layer potentials for a nonstationary linearized system Navier-Stokes equation.

3. Estimates of square functions.

4. Smallness of commutators and more Carleson measures.

5. Estimates of the $L^{2}$-norms of certain fractional derivatives of the single layer potential.

6. The estimate of a difficult term involving the pressure.

7. Estimates of the pressure.

8. Inequalities based on Rellich identities.

9. Invertibility of the single layer potential and existence results.

10. Proof of the uniqueness statements.

In Section 1 we introduce the relevant nontangential maximal functions. We also state an important result which shows how the condition imposed on $A(x, t)$ shows up in the framework of Carleson measures. In Section 2 the relevant potentials for the linearized system of Navier-Stokes equations are introduced. Mapping properties for the potentials are deduced using the results of Hofmann [18] and traces to the boundary of our potentials are considered. Some fundamental non tangential maximal function estimates for our potentials are also proved. The main purpose of Section 3 is to prove certain square function estimates for the single layer potential (Theorem 3.1). The approach is via integration by parts in "good" directions and estimates using the Carleson measure descriptions of our geometry as outlined in Section 1. Section 4 contains certain technical estimates of commutators and Carleson measures. In Section 5 we prove Theorem 5.1 which states estimates of fractional time derivatives 
of the single layer potential. This kind of estimates play an important role both in the work of Shen[32], Hofmann-Lewis[19] and Nyström[27]. In [32] the estimates are less complicated as the set up of [32] is time-independent cylinders. In our case we try to redo the calculations of Shen [32] but as our domains are time-dependent the nice cancellations used in [32] do not appear and we get complicated corrector terms. The main focus is then to prove that these corrector terms are small if $\left\|\mathbb{D}_{n} A\right\|_{*}$ is sufficently small. The main tools are partial integration, Carleson measures and estimates of certain commutators. This is combined with the estimates derived in the previous sections. In estimating the corrector terms just described one term turns out to be particularly hard. This term contains the pressure and the main obstacle is that the pressure does not behave well with respect to the parabolic nontangential maximal function. Section 6 is devoted entirely to this term. Section 7 contains some inequalities for the pressure and in Section 8 Rellich identities are explored. The definition of weak solutions and the proof of invertability of our potentials can found in Section 9. In that section the existence results of Theorem A, B and C are also treated. The proof of invertibility of the singular layer potential follows, after some additional arguments, from the inequalities proved in Section 8. In Section 10 we prove the uniqueness part of Theorem A, B and C.

1. Carleson measures and maximal functions. Our geometric set up is, as described in the intoduction, graph domains of the form

$$
\Omega=\left\{\left(x_{0}, x, t\right) \in \mathbb{R} \times \mathbb{R}^{n-1} \times \mathbb{R}: x_{0}>A(x, t)\right\}
$$

where $n \geq 2$ and the basic geometric restriction we impose on our domains is that

$$
\|A\|_{\text {comm }}=\left\|\nabla_{x} A\right\|_{\infty}+\left\|\mathbb{D}_{n} A\right\|_{*}<\infty .
$$

In the introduction we introduced $D_{1 / 2}^{t}$, the one-dimensional one half fractional derivation operator in the time variable and we mentioned that the smallness of $\left\|\mathbb{D}_{n} A\right\|_{*}$ could equivalently be stated as a smallness condition on $\left\|D_{1 / 2}^{t} A\right\|_{*}$.

We also mentioned that $\|A\|_{\text {comm }} \leq \beta<\infty$ implies that $A(x, t)$ is parabolically Lipschitz in the following sense,

$$
|A(x, t)-A(y, s)| \leq \beta\left(|x-y|+|t-s|^{1 / 2}\right) \quad x, y \in \mathbb{R}^{n} \quad t, s \in \mathbb{R} .
$$

In this section we will now state an important result which shows how the condition imposed on $A(x, t)$ shows up in the framework of Carleson measures.

Let $P(z) \in C_{0}^{\infty}\left(\mathbb{R}^{n}\right)$. We furthermore assume that $P(z)$ is a non-negative function and that $\int_{\mathbb{R}^{n}} P(z) d z=1$. I.e. we assume that $P(z)$ is a parabolic approximate identity. Let $d=n+1$ and define

$$
P_{\lambda}(z)=\lambda^{-d} P\left(\lambda^{-\alpha} z\right)=\lambda^{-d} P\left(\frac{x}{\lambda}, \frac{t}{\lambda^{2}}\right) .
$$

For a locally integrabel function $f$ we introduce the convolution operator

$$
P_{\lambda} f(z)=\int_{R^{n}} P_{\lambda}(z-v) f(v) d v .
$$

Following Dahlberg[7] and Hofmann-Lewis [19] we use a construction of Kenig-Stein to define a "parabolic" lifting $\rho(\lambda, x, t)$ from $\mathbb{R}_{+}^{n+1}=\mathbb{R}_{+} \times \mathbb{R}^{n-1} \times \mathbb{R}$ onto $\Omega=\left\{\left(x_{0}, x, t\right) \in\right.$ 
$\left.\mathbb{R} \times \mathbb{R}^{n-1} \times \mathbb{R}: x_{0}>A(x, t)\right\}$ in the following way,

$$
\rho(\lambda, x, t)=\left(\lambda+P_{\gamma \lambda} A(x, t), x, t\right) \quad \rho(0, x, t)=(A(x, t), x, t) .
$$

Here $\gamma$ is a small parameter at our disposial and we may adjust $\gamma$, as $\left\|\nabla_{x} A\right\|_{\infty}<\infty$, so that,

$$
\frac{1}{2} \leq 1+\frac{\partial P_{\gamma \lambda} A(z)}{\partial \lambda} \leq 3 / 2
$$

The following lemma is crucial and incorporates the geometrical information in an analytic and quantitative way (for a proof see [19, p.365-366]).

LEMMA 1.1. Let $\sigma, \theta$ be non-negative integers and let $\phi=\left(\phi_{1}, \ldots, \phi_{n-1}\right)$ be a multiindex. Define $\ell=\sigma+|\phi|+\theta$. If $\|A\|_{\text {comm }} \leq \beta<\infty$ and if we define measures in the following way,

$$
d v=\left(\frac{\partial^{\ell} P_{\gamma \lambda} A(x, t)}{\partial \lambda^{\sigma} \partial x^{\phi} \partial t^{\theta}}\right)^{2} \lambda^{2 \ell+2 \theta-3} d x d t d \lambda
$$

then this is a Carleson measure on $\mathbb{R}_{+}^{n+1}$ if either $\sigma+\theta \geq 1$ or $|\phi| \geq 2$ with

$$
(i) v\left(B_{r}(z) \times(0, r)\right) \leq C r^{d} \gamma^{(2-2|\phi|-4 \theta)} b^{2}(1+\beta)^{2}
$$

where $b=\left\|\mathbb{D}_{n} A\right\|_{*}$ if $\theta \geq 1$ and $b=1$, if $\theta=0$.

Moreover if $\ell \geq 1$ then

$$
\text { (ii) }\left\|\frac{\partial^{\ell} P_{\gamma \lambda} A}{\partial \lambda^{\sigma} \partial x^{\phi} \partial t^{\theta}}\right\|_{\infty} \leq C_{1} \gamma^{(1-|\phi|-2 \theta)} \lambda^{1-\ell-\theta} b(1+\beta)
$$

while if either $\sigma+\theta \geq 1$ or $|\phi| \geq 2$ then

$$
\text { (iii) } \lim _{(\lambda, y, s) \rightarrow(0, x, t)}\left(\lambda^{\ell+\theta-1} \frac{\partial^{\ell} P_{\gamma \lambda} A(y, s)}{\partial \lambda^{\sigma} \partial y^{\phi} \partial s^{\theta}}\right)=0
$$

for a.e. $(x, t) \in R^{n}$.

It is a well known fact that Carleson measures and maximal functions play an important role in harmonic analysis. In our case the important maximal function is the non tangential maximal function operator that we now intend to introduce. Let $a>0$ and $(P, t)=\left(p_{0}, p, t\right) \in \partial \Omega$. We let $\tilde{\Gamma}(P, t)=\tilde{\Gamma}_{a}(P, t)$ be the parabolic cone

$$
\tilde{\Gamma}(P, t)=\left\{\left(q_{0}, q, s\right) \in \Omega:\|(p-q, t-s)\|<a\left|q_{0}-A(p, t)\right|\right\} .
$$

We also introduce an elliptic cone,

$$
\tilde{\Gamma}_{e}(P, t)=\left\{\left(q_{0}, q, t\right) \in \Omega:|p-q|<a\left|q_{0}-A(p, t)\right|\right\} .
$$

If $h$ is a function defined on $\Omega$ we define the non-tangential maximal function $\tilde{N}_{*} h$ : $\partial \Omega \rightarrow \mathbb{R}$ by

$$
\tilde{N}_{*} h(P, t)=\sup _{(Q, s) \in \tilde{\Gamma}(P, t)}|h|(Q, s) .
$$

If $(P, t) \in \partial \Omega$ then by $\lim _{(Q, s) \rightarrow(P, t)} h(Q, s)$ we mean the limit as $(Q, s) \rightarrow(P, t)$ in $\tilde{\Gamma}(P, t)$. We furthermore define an elliptic non-tangential maximal function $\tilde{N}_{*, e} h: \partial \Omega \rightarrow \mathbb{R}$ in 
the following way,

$$
\tilde{N}_{*, e} h(P, t)=\sup _{(Q, t) \in \tilde{\Gamma}_{e}(P, t)}|h|(Q, t) .
$$

And if $(P, t) \in \partial \Omega$ then by $\lim _{(Q, t) \rightarrow(P, t)} h(Q, t)$ we mean the limit as $(Q, t) \rightarrow(P, t)$ in $\tilde{\Gamma}_{e}(P, t)$.

For a function $g$ defined on $\mathbb{R}_{+}^{n+1}$ and for $a \geq 1$ fixed we also introduce the following maximal function $N_{*} g: \mathbb{R}_{+}^{n+1} \rightarrow \mathbb{R}$,

$$
N_{*} g(x, t)=\sup _{\lambda>0,(y, s) \in B_{a \lambda}(x, t)}|g|(\lambda, y, s),
$$

where $B_{a \lambda}(x, t)$ is the parabolic ball described in the introduction. If we let $\Gamma_{a}(x, t):=\left\{(\lambda, y, s), \lambda>0,(y, s) \in B_{a \lambda}(x, t)\right\}$ one may easily proved that $\tilde{\Gamma}_{\bar{a}}(\rho(0, x, t)) \subset \rho\left(\Gamma_{a}(x, t)\right)$, for $\bar{a}$ sufficiently small depending on $a$ and $\|A\|_{\text {comm }}$. Given $a \geq 1$ we will by $\lim _{(\lambda, y, s) \rightarrow(0, x, t)}$ mean that $(\lambda, y, s) \rightarrow(0, x, t)$ in $\Gamma_{a}(x, t)$. Again we define an elliptic counterpart in the following way,

$$
N_{*, e} g(x, t)=\sup _{\lambda>0,(y, t) \in B_{a \lambda}(x, t)}|g|(\lambda, y, t) .
$$

If, as in the statement of Lemma $1.1, d \nu$ is a Carleson measure on $\mathbb{R}_{+}^{n+1}$ and $g$ : $\mathbb{R}_{+}^{n+1} \rightarrow \mathbb{R}$ then it is well known that,

$$
\int_{\mathbb{R}_{+}^{n+1}}|g|^{p} d \nu \leq C_{\nu} \int_{\mathbb{R}^{n}}\left|N_{*}(g)\right|^{p} d x d t,
$$

where $C_{\nu}$ is the Carleson norm of the measure $\nu$. I.e., $C_{\nu}$ is the smallest constant $C^{*}$ such that

$$
\nu\left(B_{r}(x, t) \times(0, r)\right) \leq C^{*} r^{d},
$$

for all $(x, t) \in \mathbb{R}^{n}$ and $r>0$. We refer the reader to Stein [29] for more information on Carleson measures. The version of $(*)$ that we will make use of in our paper is the following. We let $u$ be function defined on $\Omega$. If either $\sigma+\theta \geq 1$ or $|\phi| \geq 2$ then according to Lemma 1.1 the following is true,

$$
\begin{aligned}
& \int_{0}^{\infty} \int_{\mathbb{R}^{n}}|u \circ \rho|^{2}\left(\frac{\partial^{\ell} P_{\gamma \lambda} A(x, t)}{\partial \lambda^{\sigma} \partial x^{\phi} \partial t^{\theta}}\right)^{2} \lambda^{2 \ell+2 \theta-3} d x d t d \lambda \\
& \leq C \gamma^{(2-2|\phi|-4 \theta)} b^{2}(1+\beta)^{2} \int_{\mathbb{R}^{n}}\left|N_{*}(u \circ \rho)\right|^{2} d x d t
\end{aligned}
$$

where $b=\left\|\mathbb{D}_{n} A\right\|_{*}$ if $\theta \geq 1$ and $b=1$, if $\theta=0$.

2. Layer potentials for a nonstationary linearized system of NavierStokes equations. Let in the following $(X, t)=\left(x_{0}, x_{1}, \ldots, x_{n-1}, t\right)$, $(x, t)=\left(x_{1}, \ldots, x_{n-1}, t\right)$. Let $W(X, t)$ be the fundamental solution to the heat equation and let $\delta(t)$ be the Dirac delta function concentrated at $t=0 . w_{n}$ denotes the surface measure on the unit sphere in $\mathbb{R}^{n}$. Let $\Gamma(X, t)=\left\{\Gamma_{j k}(X, t)\right\}_{n \times n}$ denote the 
Oseen's tensor and let $\left(q^{0}(X, t), \ldots, q^{n-1}(X, t)\right)$ denote the corresponding "pressure" vector. I.e.,

$$
\begin{aligned}
\Gamma_{j k}(X, t) & =\delta_{j k} W(X, t)+\int_{t}^{\infty} \frac{\partial^{2} W(X, s)}{\partial x_{j} \partial x_{k}} d s, \\
q^{j}(X, t) & =\frac{\delta(t) x_{j}}{w_{n}|x|^{n}} .
\end{aligned}
$$

Then $\Gamma$ is a fundamental solution to the linearized Navier-Stokes equation. For $\vec{f} \in$ $L^{2}(\partial \Omega)$ we introduce,

$$
\begin{aligned}
\vec{u}(X, t)=S \vec{f}(X, t) & =\int_{-\infty}^{t} \int_{\partial \Omega_{s}} \Gamma(X-Q, t-s) \vec{f}(Q, s) d \sigma_{s}(Q) d s, \\
p(X, t) & =\int_{\partial \Omega_{t}} \frac{\langle X-Q, \vec{f}(Q, t)\rangle}{w_{n}|X-Q|^{n}} d \sigma_{t}(Q) .
\end{aligned}
$$

The pair $(\vec{u}, p)$ solves the system,

$$
\left\{\begin{array}{r}
\frac{\partial \vec{u}}{\partial t}=\Delta \vec{u}-\nabla p \\
\operatorname{div} \vec{u}=0
\end{array}\right.
$$

in $\mathbb{R}^{n+1} \backslash \partial \Omega$. Note that for each fixed $t, p_{t}(X)=p(X, t)$ is a harmonic function in $\Omega_{t}$. We introduce the following conormal derivative

$$
\frac{\partial \vec{u}}{\partial \nu}:=(\nabla \vec{u}) N-p N
$$

We first state two important results on $L^{p}$ - continuity of potentials.

Theorem 2.1. Let $\|A\|_{\text {comm }} \leq \beta<\infty$ and let $\vec{f} \in L^{p}(\partial \Omega)$ with $1<p<\infty$. Define for $(P, t) \in \partial \Omega$ and $j=0,1 \ldots, n-1$ the following operators

$$
K^{j} \vec{f}(P, t):=p . v \int_{-\infty}^{t} \int_{\partial \Omega_{s}} \frac{\partial \Gamma}{\partial x_{j}}(P-Q, t-s) \vec{f}(Q, s) d \sigma_{s}(Q) d s .
$$

Then

$$
\left\|K^{j} \vec{f}\right\|_{p} \leq C_{\beta, p}\|\vec{f}\|_{p} .
$$

Proof. Using the argument of Coifman-David-Meyer [4] the theorem is a consequence of $[18$,Theorem 1], i.e. a consequence of the results of Hofmann on parabolic singular integrals. In case of the parabolic Lamé system the argument is presented in [27]. We only need to slightly modify that argument in order to cover the proof of Theorem 2.1 .

A consequence of the theorem is that $K^{j} \vec{f}(P, t)$ exists for a.e. $(P, t) \in \partial \Omega$ w.r.t. $d \sigma_{t} d t$. 
We now consider continuity of $S_{b} \vec{f}$ in the regularity space $L_{1,1 / 2}^{2}(\partial \Omega)$. By definition,

$$
S_{b} \vec{f}(P, t)=\int_{-\infty}^{t} \int_{\partial \Omega_{s}} \Gamma(P-Q, t-s) \vec{f}(Q, s) d \sigma_{s}(Q) d s .
$$

for all $(P, t) \in \partial \Omega$ for which this expression makes sense. Recall that $L_{1,1 / 2}^{2}(\partial \Omega)$ consists of functions with distributional derivatives in $x$ satisfying $\|f\|_{L_{1,1 / 2}^{2}(\partial \Omega)}<\infty$, where $\|f\|_{L_{1,1 / 2}^{2}(\partial \Omega)}=\|\mathbb{D} \tilde{f}\|_{2}$ and $\tilde{f}=f \circ \pi^{-1}, \pi(A(x, t), x, t)=(x, t)$. We have, by applying Plancherel's theorem, that

$$
\|\mathbb{D} \tilde{f}\|_{2} \approx\left\|D_{1 / 2}^{t} \tilde{f}\right\|_{2}+\left\|\nabla_{x} \tilde{f}\right\|_{2}
$$

where $D_{1 / 2}^{t}$ denotes the one-dimensional one half fractional derivative of $\tilde{f}$ in the time variable.

Using [18, Theorem 2] of Hofmann and standard arguments one may prove the following continuity result for $S_{b} \vec{f}$ in the space $L_{1,1 / 2}^{2}(\partial \Omega)$. Again the argument is presented in case of the parabolic Lamé system in [27].

Theorem 2.2. Let $\|A\|_{\text {comm }} \leq \beta<\infty$ and let $\vec{f} \in L^{2}(\partial \Omega)$. Then

$$
\left\|S_{b} \vec{f}\right\|_{L_{1,1 / 2}^{2}(\partial \Omega)} \leq C_{\beta}\|\vec{f}\|_{2} .
$$

We will frequently make use of the following interior regularity estimate.

Lemma 2.3. Let the pair $(\vec{u}, p)$ solve the linearized system of Navier-Stokes in the parabolic cylinder $B_{2 r}(0) \times\left(-4 r^{2}, 4 r^{2}\right)$. Then

$$
\int_{B_{r / 2}(0) \times\left(-\frac{1}{4} r^{2}, \frac{1}{4} r^{2}\right)}|\nabla \vec{u}|^{2} d x d t \leq \frac{c}{r^{2}} \int_{B_{r}(0) \times\left(-r^{2}, r^{2}\right)}\left(|\vec{u}|^{2}+|p|^{2}\right) d x d t
$$

Proof. Let $r=1$ by rescaling and let $\varphi \in C_{0}^{\infty}\left(B_{1}(0) \times(-1,1)\right), \varphi \equiv 1$ on $B_{3 / 4}(0) \times\left(-\left(\frac{3}{4}\right)^{2},\left(\frac{3}{4}\right)^{2}\right)$. Then

$$
\int_{B_{1}(0) \times(-1,1)}\left\langle\vec{u}_{t}, \vec{u}\right\rangle \varphi^{2} d X d t=\int_{B_{1}(0) \times(-1,1)}\langle\Delta \vec{u}, \vec{u}\rangle \varphi^{2} d X d t-\int_{B_{1}(0) \times(-1,1)}\langle\nabla p, \vec{u}\rangle \varphi^{2} d X d t .
$$

Integrating by parts we have

$$
\begin{gathered}
-\frac{1}{2} \int_{B_{1}(0) \times(-1,1)}|\vec{u}|^{2} \varphi \frac{\partial \varphi}{\partial t} d X d t=-\int_{B_{1}(0) \times(-1,1)}|\nabla \vec{u}|^{2} \varphi^{2} d X d t \\
+2 \sum_{i=0}^{n-1} \int_{B_{1}(0) \times(-1,1)}\left\langle\nabla u^{i}, \nabla \varphi\right\rangle u^{i} \varphi d X d t-\int_{B_{1}(0) \times(-1,1)}\langle\nabla p, \vec{u}\rangle \varphi^{2} d X d t .
\end{gathered}
$$

As $\operatorname{div} \vec{u}=0$,

$$
-\int_{B_{1}(0) \times(-1,1)}\langle\nabla p, \vec{u}\rangle \varphi^{2} d X d t=\int_{B_{1}(0) \times(-1,1)}\left\langle\nabla \varphi^{2}, \vec{u}\right\rangle p d X d t .
$$


The conclusion now follows from simple manipulations.

We now define the relevant double layer potential of $\vec{f} \in L^{2}(\partial \Omega)$ in the following way,

$$
\begin{aligned}
\vec{v}(X, t)=D \vec{f}(X, t) & :=\int_{-\infty}^{t} \int_{\partial \Omega_{s}} \frac{\partial \Gamma}{\partial N_{s}(Q)}(X-Q, t-s) \vec{f}(Q, s) d \sigma_{s}(Q) d s \\
& -\int_{\partial \Omega_{t}} \frac{Q-X}{w_{n}|X-Q|^{n}}\left\langle\vec{f}(Q, t), N_{t}(Q)\right\rangle d \sigma_{t}(Q) .
\end{aligned}
$$

The following lemma contains crucial estimates that will be used frequently in the forthcoming sections.

Lemma 2.4. Let $\|A\|_{\text {comm }} \leq \beta$ and let $\vec{f} \in L^{2}(\partial \Omega)$. Then

$$
\begin{aligned}
& (i)\left\|N_{*, e}(D \vec{f} \circ \rho)\right\|_{2} \leq C_{\beta}\|\vec{f}\|_{2}, \\
& (i i)\left\|N_{*}(\nabla S \vec{f} \circ \rho)\right\|_{2} \leq C_{\beta}\|\vec{f}\|_{2}, \\
& (i i i)\left\|N_{*}\left(H D_{1 / 2}^{t}(S \vec{f} \circ \rho)\right)\right\|_{2} \leq C\left(1+\gamma^{-1}\left\|\mathbb{D}_{n} A\right\|_{*}\right)\|\vec{f}\|_{2} .
\end{aligned}
$$

Proof. (i) and (ii) are proved by standard arguments. Left to prove is (iii). The following is an adaption of the argument of Hofmann-Lewis [19,p.367-371]. At one important step the argument below is fundamentally different from the argument in [19] as well as the argument in [27]. The reason for this is the poor continuity of the pressure in the time-direction.

Let $(x, t) \in \mathbb{R}^{n}$. Fix $a \geq 1, K \geq 2, \lambda>0$ and let $(\tilde{x}, \tilde{t}) \in B_{a \lambda}(x, t)$. Let $\vec{u}=S \vec{f}$. Then,

$$
\begin{aligned}
H D_{1 / 2}^{t}(\vec{u} \circ \rho)(\lambda, \tilde{x}, \tilde{t}) & =\lim _{\varepsilon \rightarrow 0} \int_{\varepsilon<|s-\tilde{t}|<1 / \varepsilon} \frac{\operatorname{sign}(\tilde{t}-s)}{|\tilde{t}-s|^{3 / 2}(\vec{u} \circ \rho)(\lambda, \tilde{x}, s) d s} \\
& =\lim _{\varepsilon \rightarrow 0} \int_{\left\{\varepsilon \leq|s-\tilde{t}|<(K a \lambda)^{2}\right\}} \frac{\operatorname{sign}(\tilde{t}-s)}{|\tilde{t}-s|^{3 / 2}}(\vec{u} \circ \rho)(\lambda, \tilde{x}, s) d s \\
& +\lim _{\varepsilon \rightarrow 0} \int_{\left\{(K a \lambda)^{2} \leq|s-\tilde{t}|<1 / \varepsilon\right\}} \frac{\operatorname{sign}(\tilde{t}-s)}{|\tilde{t}-s|^{3 / 2}}(\vec{u} \circ \rho)(\lambda, \tilde{x}, s) d s \\
& =g_{1}(\lambda, \tilde{x}, \tilde{t})+g_{2}(\lambda, \tilde{x}, \tilde{t}) .
\end{aligned}
$$

Note that if we define

$$
g_{3}(\lambda, \tilde{x}, \tilde{t})=\sup _{\left\{\tau:|\tau-\tilde{t}| \leq(2 K a \lambda)^{2}\right\}}\left|(\vec{u} \circ \rho)_{\tau}(\lambda, \tilde{x}, \tau)\right|
$$

then

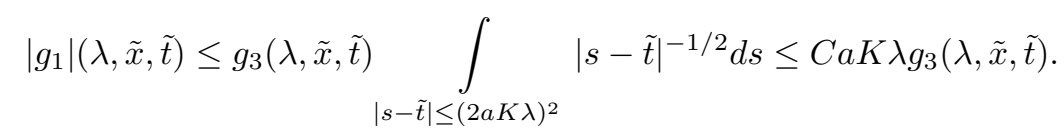

Furthermore, if $(\tau-\tilde{t}) \leq(2 K a \lambda)^{2}$, then at $(\lambda, \tilde{x}, \tau)$,

$$
(\vec{u} \circ \rho)_{\tau}=\left(\vec{u}_{\tau} \circ \rho\right)+\left(\vec{u}_{\tilde{x}_{0}} \circ \rho\right) \frac{\partial P_{\gamma \lambda} A}{\partial \tau},
$$


and by applying Lemma 1.1 we have

$$
\left|\left(\vec{u}_{\tilde{x}_{0}} \circ \rho\right)\left(\frac{\partial P_{\gamma \lambda} A}{\partial \tau}\right)(\lambda, \tilde{x}, \tau)\right| \leq C_{a, K, \beta}(\gamma \lambda)^{-1}\left\|\mathbb{D}_{n} A\right\|_{*} N_{* *}\left(\vec{u}_{\tilde{x}_{0}} \circ \rho\right)(x, t),
$$

where $N_{* *}$ is the nontangential maximal function defined relative to $\Gamma_{c_{1} K a}(x, t)$, where $c_{1}$ is so large that $(\lambda, \tilde{x}, \tau) \in \Gamma_{c_{1} K a}(x, t)$.

We need to estimate $\left|\vec{u}_{\tau} \circ \rho(\lambda, \tilde{x}, \tau)\right|$. Using the equation as in Hofmann-Lewis[19] and Nyström[27] we will run into trouble because of the poor continuity of the pressure in the time-direction. Instead we use the homogenity of the kernel of $S \vec{f}$, i.e. the homogenity of $\Gamma(X, t)$. Recall that

$$
\Gamma\left(\delta X, \delta^{2} t\right)=\delta^{-n} \Gamma(X, t)=\delta^{-d+1} \Gamma(X, t) .
$$

I.e, the kernel is homogenous of degree $d-1$. The kernel of $\vec{u}_{\tau}$ is therefore homogenous of degree $d+1$. Using this we get, after some fairly standard arguments that,

$$
\left|\vec{u}_{\tau} \circ \rho(\lambda, \tilde{x}, \tau)\right| \leq C \lambda^{-1} M(\vec{f})(x, t) .
$$

Here $M$ is a parabolic Hardy-Littlewood maximal function.

Summarizing we have proved the following estimate of $g_{3}(\lambda, \tilde{x}, \tilde{t})$ when $(\tilde{x}, \tilde{t}) \in B_{a \lambda}(x, t)$.

$$
\left|g_{3}(\lambda, \tilde{x}, \tilde{t})\right| \leq C_{a, K, \beta}\left[(\gamma \lambda)^{-1}\left\|\mathbb{D}_{n} A\right\|_{*} N_{* *}\left(\vec{u}_{\tilde{x}_{0}} \circ \rho\right)(x, t)+\lambda^{-1} M(\vec{f})(x, t)\right] .
$$

Using part (ii) of the lemma and continuity of the maximal function we may conclude that

$$
\left\|N_{*}\left(g_{1}\right)\right\|_{2} \leq C\|\vec{f}\|_{2}\left[1+\gamma^{-1}\left\|\mathbb{D}_{n} A\right\|_{*}\right] .
$$

We therefore have the desired estimate for $g_{1}$.

To estimate $g_{2}(\lambda, \tilde{x}, \tilde{t})$ we do study the function

$$
g_{4}(\lambda, \bar{x}, \bar{t}):=\lim _{\varepsilon \rightarrow 0} \int_{\left\{(k a \lambda)^{2}<|s-\bar{t}|<1 / \varepsilon\right\}} \frac{\operatorname{sign}(\bar{t}-s)}{|\bar{t}-s|^{3 / 2}}(\vec{u} \circ \rho)(0, \bar{x}, s) d s .
$$

From Sobolev type estimates we conclude that $g_{4}(\lambda, \bar{x}, \bar{t})$ is well defined for a.e. $(\bar{x}, \bar{t}) \in$ $\mathbb{R}^{n}$. Arguing as in $[19, \mathrm{p} .368]$ we have, as $|x-\tilde{x}|+|t-\tilde{t}|^{1 / 2} \leq c a \lambda$,

$$
\begin{gathered}
\left|g_{2}(\lambda, \tilde{x}, \tilde{t})-g_{4}(\lambda, x, t)\right| \leq \\
c_{a, \beta} K^{-1}\left(\sum_{i=0}^{n-1} M_{n}\left(N_{*}(\vec{u} \circ \rho)_{x_{i}}\right)(x, t)+\lambda M_{n}^{(2)}\left((\vec{u} \circ \rho)_{\tilde{t}}\right)(\lambda, \tilde{x}, \tilde{t})\right),
\end{gathered}
$$

where $M_{n}$ denotes the one-dimensional Hardy-Littlewood maximal function in the $t$-variable, while the other variable is held constant and $M_{n}^{(2)}=M_{n} \circ M_{n}$. With the same deductions as above, in particularly noting the estimate of $\vec{u}_{t}$, we get

$$
\lambda(\vec{u} \circ \rho)_{\tilde{t}}(\lambda, \tilde{x}, \tilde{t}+\xi) \leq c_{a, \beta}\left(M(\vec{f})(x, t+\xi)+\gamma^{-1}\left\|\mathbb{D}_{n} A\right\|_{*} \times\left(\sum_{i=0}^{n-1} \bar{N}_{*}\left(\left(\vec{u}_{x_{i}} \circ \rho\right)\right)(x, t+\xi)\right)\right)
$$


where $\bar{N}_{*}$ is defined relative to $\Gamma_{c a}$. Note again that for $0 \leq i \leq n-1$,

$$
(\vec{u} \circ \rho)_{x_{i}}=\left(\vec{u}_{x_{0}} \circ \rho\right) \frac{\partial P_{\gamma \lambda} A}{\partial x_{i}}+\left(\vec{u}_{x_{i}} \circ \rho\right) .
$$

Using Lemma 1.1 once again and the inequality above we deduce

$$
\begin{gathered}
N_{*} g_{2}(x, t) \leq \sup _{\lambda>0} g_{4}(\lambda, x, t)+K^{-1} C_{a, \beta}\left(1+\gamma^{-1}\left\|\mathbb{D}_{n} A\right\|_{*}\right) \sum_{i=0}^{n-1} M_{n}^{(2)}\left(\bar{N}_{*}\left(\vec{u}_{x_{i}} \circ \rho\right)\right)(x, t) \\
\quad+K^{-1} C_{a, \beta} M_{n}^{(2)}(M(\vec{f}))(x, t) \\
\end{gathered}
$$

Define $\psi(x, t)=\sup _{\lambda>0}\left|g_{4}(\lambda, x, t)\right|$ whenever $(x, t) \in \mathbb{R}^{n}$. We have from Part (ii) of our lemma and the Hardy-Littlewood maximal function theorem that

$$
\left\|N_{*} g_{2}\right\|_{2} \leq K^{-1} C_{a, \beta}\left(1+\gamma^{-1}\left\|\mathbb{D}_{n} A\right\|_{*}\right)\|\vec{f}\|_{2}+\|\psi\|_{2}
$$

It therefore sufficies to prove the estimate

$$
\|\psi\|_{2} \leq C_{a, \beta}\|\vec{f}\|_{2}
$$

But this estimate follows from the argument presented in [19, p.369-370]. The proof is therefore complete.

We will now consider traces to the boundary of $\partial \Omega$ of the potentials under consideration. Recall that $N_{t}(P, t)$ is the unit outer normal to $\partial \Omega_{t}$ at $(P, t) \in \partial \Omega_{t}$. The normal exists a.e w.r.t the projective surface measure.

Lemma 2.5. Let $\vec{f} \in L^{2}(\partial \Omega), a>0$ and $j, k \in\{0, \cdots n-1\}$. Let $u^{j}=(S \vec{f})^{j}$. Then for a.e. $(x, t) \in \mathbb{R}^{n}$,

$$
\begin{gathered}
\lim _{(\lambda, y, s) \rightarrow(0, x, t)} \frac{\partial u^{j}}{\partial x_{k}} \circ \rho(\lambda, y, s)=\frac{1}{2}\left\{N_{t}^{k} f^{j}-N_{t}^{k} N_{t}^{j}\left\langle N_{t}, \vec{f}\right\rangle\right\} \\
+p . v \int_{-\infty}^{t} \int_{\partial \Omega_{s}} \frac{\partial \Gamma_{j r}}{\partial x_{k}}(P-Q, t-s) f^{r}(Q, s) d \sigma_{s}(Q) d s
\end{gathered}
$$

and

$$
\lim _{(\lambda, y, t) \rightarrow(0, x, t)}^{e} p \circ \rho(\lambda, y, t)=-\frac{1}{2}\left\langle N_{t}, \vec{f}\right\rangle+p . v \int_{\partial \Omega_{t}} \frac{\langle P-Q, \vec{f}(Q, t)\rangle}{w_{n}|P-Q|^{n}} d \sigma_{t}(Q) .
$$

In the first terms in these two expressions $\vec{f}$ is evaluated at the point $(P, t)=(A(x, t), x, t) . N_{t}$ is the unit normal at $(A(x, t), x, t)$.

Proof. The proof follows from standard arguments. See [25] in case of the heat operator.

We know that there exists a basis for the tangent space of $\partial \Omega_{t}$ at $(P, t)$. Let $\left\{T_{j}\right\}_{j=1}^{n-1}=\left\{T_{j}(P, t)\right\}_{j=1}^{n-1}$ be such a basis. Using Lemma 2.4 and following [19,25] one may prove the following for appropriate operators $K_{\nu}$ and $\tilde{K}_{\nu}$. 
Lemma 2.6. Let $\vec{f} \in L^{2}(\partial \Omega)$. Fix $a>0.1 \leq j \leq n-1$. Then for a.e. $(x, t) \in \mathbb{R}^{n}$ we have

(i) $\lim _{(\lambda, y, t) \rightarrow(0, x, t)}^{e} D \vec{f} \circ \rho(\lambda, y, t)=\left(-\frac{1}{2} f+\tilde{K}_{\nu} \vec{f}\right) \circ \rho(0, x, t)$,

(ii)

$$
\begin{aligned}
& \lim _{(\lambda, y, t) \rightarrow(0, x, t)}^{e}\left[\left\langle\left((\nabla S \vec{f}) \circ \rho(\lambda, y, t), N_{t} \circ \rho(0, x, t)\right)\right\rangle-(p \circ \rho(\lambda, y, t)) \cdot\left(N_{t} \circ \rho(0, x, t)\right)\right] \\
& =\left(\frac{1}{2} f+K_{\nu} \vec{f}\right) \circ \rho(0, x, t),
\end{aligned}
$$

(iii) $\lim _{(\lambda, y, s) \rightarrow(0, x, t)}\left\langle\nabla(S \vec{f}) \circ \rho(\lambda, y, s), T_{j} \circ \rho(0, x, t)\right\rangle=[(S \vec{f}) \circ \rho(0, x, t)]_{x_{j}}$,

(iv) $\lim _{(\lambda, y, s) \rightarrow(0, x, t)} H D_{1 / 2}^{t}(S \vec{f} \circ \rho)(\lambda, y, s)=H D_{1 / 2}^{t}(S \vec{f} \circ \rho)(0, x, t)$.

Now let $\rho_{-}(\lambda, x, t)=\rho(-\lambda, x, t)$ when $(\lambda, x, t) \in \mathbb{R}_{-}^{n+1}=\{(\lambda, x, t)=\lambda<0$ and $\left.(x, t) \in \mathbb{R}^{n}\right\}$. Then $\rho_{-}$maps $\mathbb{R}_{-}^{n+1}$ onto $\mathbb{R}^{n+1} \backslash \Omega$ and $\rho_{-}$extends continuously to the closure of $\mathbb{R}_{-}^{n+1}$ by putting $\rho_{-}=\rho$ on the boundary of the domain. Non-tangential cones and maximal functions are naturally defined. In the same way as the lemmas above are proved one may prove the following

Lemma 2.7. Let $\vec{f} \in L^{2}(\partial \Omega)$ and let $\vec{u}_{-}=\left.S \vec{f}\right|_{\Omega^{-}}$. Fix $a>0.1 \leq j \leq n-1$. Then for a.e. $(x, t) \in \mathbb{R}^{n}$ we have

$$
\lim _{(\lambda, y, t) \rightarrow(0, x, t)}^{e}(D \vec{f})_{-} \circ \rho_{-}(\lambda, y, s)=\left(\frac{1}{2} f+\tilde{K}_{\nu} f\right) \circ \rho_{-}(0, x, t),
$$

$$
\begin{aligned}
& \left.\left.\lim _{(\lambda, y, t) \rightarrow(0, x, t)}^{e}\left[\left\langle\left(\nabla \vec{u}_{-}\right)\right) \circ \rho_{-}(\lambda, y, t), N_{t} \circ \rho_{-}(0, x, t)\right)\right\rangle-\left(p_{-} \circ \rho_{-}(\lambda, y, t)\right) \cdot\left(N_{t} \circ \rho_{-}(0, x, t)\right)\right] \\
& =\left(-\frac{1}{2} f+K_{\nu} f\right) \circ \rho_{-}(0, x, t),
\end{aligned}
$$

(iii) $\lim _{(\lambda, y, s) \rightarrow(0, x, t)}\left\langle\nabla\left(\vec{u}_{-}\right) \circ \rho_{-}(\lambda, y, s), T_{j} \circ \rho_{-}(0, x, t)\right\rangle=\left[\left(\vec{u}_{-}\right) \circ \rho_{-}(0, x, t)\right]_{x_{j}}$,

(iv) $\lim _{(\lambda, y, s) \rightarrow(0, x, t)} H D_{1 / 2}^{t}\left(\vec{u}_{-} \circ \rho_{-}\right)(\lambda, y, s)=H D_{1 / 2}^{t}\left(\vec{u}_{-} \circ \rho_{-}\right)(0, x, t)$.

3. Estimates of square functions. This section is devoted to the proof of the following two results.

Theorem 3.1. Let $\|A\|_{\text {comm }} \leq \beta<\infty$ and $\vec{f} \in L^{2}(\partial \Omega)$. Let $S \vec{f}$ be the single layer potential associated to the linearized system of Navier-Stokes. Assume that $\gamma \leq$ $1 / 2$ and $b=\left\|\mathbb{D}_{n} A\right\|_{*} \leq \epsilon_{0}=\gamma^{8+d}$. Then

$$
\begin{aligned}
& \text { (i) } \int_{0}^{\infty} \int_{\mathbb{R}^{n}}\left|\vec{u}_{x_{i} x_{j}} \circ \rho\right|^{2} \lambda d z d \lambda \leq c_{\beta}\|\vec{f}\|_{2}^{2}, \quad 0 \leq i, j \leq n-1, \\
& \text { (ii) } \int_{0}^{\infty} \int_{\mathbb{R}^{n}}\left|\vec{u}_{x_{i} t} \circ \rho\right|^{2} \lambda^{3} d z d \lambda \leq c_{\beta}\|\vec{f}\|_{2}^{2}, \quad 0 \leq i \leq n-1,
\end{aligned}
$$


(iii) $\int_{0}^{\infty} \int_{\mathbb{R}^{n}}\left|D_{1 / 2}^{t}\left(\vec{u}_{x_{j}} \circ \rho\right)\right|^{2} \lambda d z d \lambda \leq c_{\beta}\|\vec{f}\|_{2}^{2}, \quad 0 \leq j \leq n-1$.

Corollary 3.2. Let $\|A\|_{\text {comm }} \leq \beta<\infty$ and $\vec{f} \in L^{2}(\partial \Omega)$. Let $\vec{v}=D \vec{f}$ be the double layer potential of $\vec{f}$. Then,

$$
\int_{0}^{\infty} \int_{\mathbb{R}^{n}}\left|v_{x_{j}} \circ \rho\right|^{2} \lambda d z d \lambda \leq c_{\beta}\|\vec{f}\|_{2}^{2}, \quad 0 \leq j \leq n-1 .
$$

Proof. $D \vec{f}=D_{1} \vec{f}+D_{2} \vec{f}$ where $D_{2} \vec{f}$, for each $t$, is the gradient of a single layer potential associated to the Laplace operator. $D_{1} \vec{f}$ is treated using Theorem 3.1 and $D_{2} \vec{f}$ is treated using square function estimates for the single layer potential associated to the Laplace operator in an argument similar to the one carried out in the proof of Lemma 3.3 below.

Recall that the pressure

$$
p(X, t)=\int_{\partial \Omega_{t}} \frac{\langle X-Q, \vec{f}(Q, t)\rangle}{w_{n}|X-Q|^{n}} d \sigma_{t}(Q)
$$

is harmonic as a function of the space variables for fixed t. We will need the following lemma,

Lemma 3.3. Let the pressure be defined as above with $\vec{f} \in L^{2}(\partial \Omega)$. Then

$$
\int_{0}^{\infty} \int_{\mathbb{R}^{n}}|\nabla p \circ \rho|^{2} \lambda d z d \lambda \leq C \int_{\mathbb{R}^{n}}|p \circ \rho(0, x, t)|^{2} d z \leq C \int_{\partial \Omega}|\vec{f}|^{2} d \sigma_{t} d t .
$$

Proof.

$$
\begin{gathered}
\int_{0}^{\infty} \int_{\mathbb{R}^{n}}|\nabla p \circ \rho|^{2} \lambda d z d \lambda=\int_{-\infty}^{\infty} \int_{0}^{\infty} \int_{\mathbb{R}^{n-1}}|\nabla p \circ \rho|^{2} \lambda d x d \lambda d t \\
\leq C \int_{-\infty}^{\infty}\left(\int_{\Omega_{t}}|\nabla p|^{2} \delta\left(\left(x_{0}, x\right), \partial \Omega_{t}\right) d x_{0} d x\right) d t \leq C \int_{\mathbb{R}^{n}}|p \circ \rho(0, x, t)|^{2} d z .
\end{gathered}
$$

Here we have used the result of [8][30] on the solvability of the Dirichlet problem for the Laplace operator on Lipschitz domains as well as a square function estimate.

LEMmA 3.4. Let $r, i \in\{0, \ldots, n-1\}, E=\left(1+\frac{\left.\partial P_{\gamma \lambda} A\right)}{\partial \lambda}\right)^{2} /\left(1+\left|\nabla_{x} P_{\gamma \lambda} A\right|^{2}\right)$ and $\vec{u}=S \vec{f}$ with $\vec{f} \in L^{2}(\partial \Omega)$. Let $p$ be the associated pressure. Define,

$$
A_{r, i}:=\int_{0}^{\infty} \int_{\mathbb{R}^{n}} E\left(u_{x_{i}}^{r} \circ \rho\right)\left(\frac{\partial^{2} p}{\partial x_{r} \partial x_{i}} \circ \rho\right) \lambda d z d \lambda .
$$

There exits a constant $C=C_{\beta}$ such that,

$$
\sum_{r=0}^{n-1} \sum_{i=0}^{n-1}\left|A_{r, i}\right| \leq C\left[\|\vec{f}\|_{2}^{2}+\|\vec{f}\|_{2}\left(\sum_{r, i, j=0}^{n-1} \int_{0}^{\infty} \int_{\mathbb{R}^{n}}\left|u_{x_{i} x_{j}}^{r} \circ \rho\right|^{2} \lambda d z d \lambda\right)^{1 / 2}\right] .
$$


Proof. Recall that for fixed $t, p_{t}(X)=p(X, t)$ is harmonic. Let $i \neq 0$. Then,

$$
\begin{aligned}
& \left(\frac{\partial p}{\partial x_{r}} \circ \rho\right)_{x_{i}}=\left(\frac{\partial^{2} p}{\partial x_{r} \partial x_{0}} \circ \rho\right) \frac{\partial P_{\gamma \lambda} A}{\partial x_{i}}+\left(\frac{\partial^{2} p}{\partial x_{r} \partial x_{i}} \circ \rho\right) \\
& \left(\frac{\partial p}{\partial x_{0}} \circ \rho\right)_{x_{r}}=\left(\frac{\partial^{2} p}{\partial x_{0}^{2}} \circ \rho\right) \frac{\partial P_{\gamma \lambda} A}{\partial x_{r}}+\left(\frac{\partial^{2} p}{\partial x_{r} \partial x_{0}} \circ \rho\right) .
\end{aligned}
$$

I.e.,

$\left(\frac{\partial p}{\partial x_{r}} \circ \rho\right)_{x_{i}}-\frac{\partial P_{\gamma \lambda} A}{\partial x_{i}}\left(\frac{\partial p}{\partial x_{0}} \circ \rho\right)_{x_{r}}=\left(\frac{\partial^{2} p}{\partial x_{r} \partial x_{i}} \circ \rho\right)-\left(\frac{\partial^{2} p}{\partial x_{0}^{2}} \circ \rho\right) \frac{\partial P_{\gamma \lambda} A}{\partial x_{r}} \frac{\partial P_{\gamma \lambda} A}{\partial x_{i}}$.

Using this identity to express $\left(\frac{\partial^{2} p}{\partial x_{r} \partial x_{i}} \circ \rho\right)$ we have for $i \neq 0, r \neq 0$,

$$
\begin{aligned}
A_{r, i} & =\int_{0}^{\infty} \int_{\mathbb{R}^{n}} E\left(u_{x_{i}}^{r} \circ \rho\right)\left(\frac{\partial p}{\partial x_{r}} \circ \rho\right)_{x_{i}} \lambda d z d \lambda \\
& -\int_{0}^{\infty} \int_{\mathbb{R}^{n}} E\left(u_{x_{i}}^{r} \circ \rho\right) \frac{\partial P_{\gamma \lambda} A}{\partial x_{i}}\left(\frac{\partial p}{\partial x_{0}} \circ \rho\right)_{x_{r}} \lambda d z d \lambda \\
& +\int_{0}^{\infty} \int_{\mathbb{R}^{n}} E\left(u_{x_{i}}^{r} \circ \rho\right)\left(\frac{\partial^{2} p}{\partial x_{0}^{2}} \circ \rho\right) \frac{\partial P_{\gamma \lambda} A}{\partial x_{r}} \frac{\partial P_{\gamma \lambda} A}{\partial x_{i}} \lambda d z d \lambda \\
& =-\int_{0}^{\infty} \int_{\mathbb{R}^{n}}\left(E\left(u_{x_{i}}^{r} \circ \rho\right)\right)_{x_{i}}\left(\frac{\partial p}{\partial x_{r}} \circ \rho\right) \lambda d z d \lambda \\
& +\int_{0}^{\infty} \int_{\mathbb{R}^{n}}\left(E\left(u_{x_{i}}^{r} \circ \rho\right) \frac{\partial P_{\gamma \lambda} A}{\partial x_{i}}\right)_{x_{r}}\left(\frac{\partial p}{\partial x_{0}} \circ \rho\right)_{x_{r}} \lambda d z d \lambda \\
& +\int_{0}^{\infty} \int_{\mathbb{R}^{n}} E\left(u_{x_{i}}^{r} \circ \rho\right)\left(\frac{\partial^{2} p}{\partial x_{0}^{2}} \circ \rho\right) \frac{\partial P_{\gamma \lambda} A}{\partial x_{r}} \frac{\partial P_{\gamma \lambda} A}{\partial x_{i}} \lambda d z d \lambda .
\end{aligned}
$$

Obviously by definition,

$$
A_{0,0}=\int_{0}^{\infty} \int_{\mathbb{R}^{n}} E\left(u_{x_{0}}^{0} \circ \rho\right)\left(\frac{\partial^{2} p}{\partial x_{0}^{2}} \circ \rho\right) \lambda d z d \lambda
$$

We now let $i=0$ and $r \neq 0$. We need to manipulate $\left(\frac{\partial^{2} p}{\partial x_{r} \partial x_{0}} \circ \rho\right)$. But

$$
\left(\frac{\partial p}{\partial x_{0}} \circ \rho\right)_{x_{r}}=\left(\frac{\partial^{2} p}{\partial x_{0}^{2}} \circ \rho\right) \frac{\partial P_{\gamma \lambda} A}{\partial x_{r}}+\left(\frac{\partial^{2} p}{\partial x_{r} \partial x_{0}} \circ \rho\right) .
$$

We therefore have,

$$
A_{r, 0}=\int_{0}^{\infty} \int_{\mathbb{R}^{n}} E\left(u_{x_{0}}^{r} \circ \rho\right)\left(\frac{\partial p}{\partial x_{0}} \circ \rho\right)_{x_{r}} \lambda d z d \lambda
$$




$$
\begin{aligned}
& -\int_{0}^{\infty} \int_{\mathbb{R}^{n}} E\left(u_{x_{0}}^{r} \circ \rho\right)\left(\frac{\partial^{2} p}{\partial x_{0}^{2}} \circ \rho\right) \frac{\partial P_{\gamma \lambda} A}{\partial x_{r}} \lambda d z d \lambda \\
& =-\int_{0}^{\infty} \int_{\mathbb{R}^{n}}\left(E\left(u_{x_{0}}^{r} \circ \rho\right)\right)_{x_{r}}\left(\frac{\partial p}{\partial x_{0}} \circ \rho\right) \lambda d z d \lambda \\
& -\int_{0}^{\infty} \int_{\mathbb{R}^{n}} E\left(u_{x_{0}}^{r} \circ \rho\right)\left(\frac{\partial^{2} p}{\partial x_{0}^{2}} \circ \rho\right) \frac{\partial P_{\gamma \lambda} A}{\partial x_{r}} \lambda d z d \lambda .
\end{aligned}
$$

i.e., using the Carleson measure characterizations of Lemma 1.1 we have

$$
\begin{aligned}
\sum_{r, i=0}^{n-1}\left|A_{r, i}\right| \leq C & {\left[\left\|N_{*}\left(u_{x_{i}}^{r} \circ \rho\right)\right\|_{2}\left(\int_{0}^{\infty} \int_{\mathbb{R}^{n}}|\nabla p \circ \rho|^{2} \lambda d z d \lambda\right)^{1 / 2}\right.} \\
& \left(\sum_{j=0}^{n-1} \int_{0}^{\infty} \int_{\mathbb{R}^{n}}\left|u_{x_{i} x_{j}}^{r} \circ \rho\right|^{2} \lambda d z d \lambda\right)^{1 / 2}\left(\int_{0}^{\infty} \int_{\mathbb{R}^{n}}|\nabla p \circ \rho|^{2} \lambda d z d \lambda\right)^{1 / 2} \\
& +\sum_{r, i=0}^{n-1}\left|\int_{0}^{\infty} \int_{\mathbb{R}^{n}} E\left(u_{x_{i}}^{r} \circ \rho\right)\left(\frac{\partial^{2} p}{\partial x_{0}^{2}} \circ \rho\right) \frac{\partial P_{\gamma \lambda} A}{\partial x_{r}} \frac{\partial P_{\gamma \lambda} A}{\partial x_{i}} \lambda d z d \lambda\right| \\
& +\sum_{r=0}^{n-1}\left|\int_{0}^{\infty} \int_{\mathbb{R}^{n}} E\left(u_{x_{0}}^{r} \circ \rho\right)\left(\frac{\partial^{2} p}{\partial x_{0}^{2}} \circ \rho\right) \frac{\partial P_{\gamma \lambda} A}{\partial x_{r}} \lambda d z d \lambda\right| \\
& \left.+\left|\int_{0}^{\infty} \int_{\mathbb{R}^{n}} E\left(u_{x_{0}}^{0} \circ \rho\right)\left(\frac{\partial^{2} p}{\partial x_{0}^{2}} \circ \rho\right) \lambda d z d \lambda\right|\right] .
\end{aligned}
$$

Using Lemma 2.4 and Lemma 3.3 the first two products are controlled. We therefore have to handle terms of the form,

$$
\int_{0}^{\infty} \int_{\mathbb{R}^{n}} F\left(u_{x_{i}}^{r} \circ \rho\right)\left(\frac{\partial^{2} p}{\partial x_{0}^{2}} \circ \rho\right) \lambda d z d \lambda
$$

where $F$ is a function containing derivatives of the defining function for the domain. Using that $p$ is harmonic for each fixed $t$ we have

$$
\begin{aligned}
\frac{\partial^{2} p}{\partial x_{0}^{2}} \circ \rho & =-\sum_{j=1}^{n-1} \frac{\partial^{2} p}{\partial x_{j}^{2}} \circ \rho \\
& =-\sum_{j=1}^{n-1}\left(\left(\frac{\partial p}{\partial x_{0}} \circ \rho\right)_{x_{j}} \frac{\partial P_{\gamma \lambda} A}{\partial x_{j}}-\left(\frac{\partial p}{\partial x_{j}} \circ \rho\right)_{x_{j}}\right)-\left|\nabla P_{\gamma \lambda} A\right|^{2}\left(\frac{\partial^{2} p}{\partial x_{0}^{2}} \circ \rho\right) .
\end{aligned}
$$

I.e.,

$$
\left(1+\left|\nabla P_{\gamma \lambda} A\right|^{2}\right)\left(\frac{\partial^{2} p}{\partial x_{0}^{2}} \circ \rho\right)=\sum_{j=1}^{n-1}\left(-\left(\frac{\partial p}{\partial x_{0}} \circ \rho\right)_{x_{j}} \frac{\partial P_{\gamma \lambda} A}{\partial x_{j}}+\left(\frac{\partial p}{\partial x_{j}} \circ \rho\right)_{x_{j}}\right) .
$$


Therefore,

$$
\begin{aligned}
& \int_{0}^{\infty} \int_{\mathbb{R}^{n}} F\left(u_{x_{i}}^{r} \circ \rho\right)\left(\frac{\partial^{2} p}{\partial x_{0}^{2}} \circ \rho\right) \lambda d z d \lambda \\
= & \int_{0}^{\infty} \int_{\mathbb{R}^{n}} \frac{F\left(u_{x_{i}}^{r} \circ \rho\right)}{\left(1+\left|\nabla P_{\gamma \lambda} A\right|^{2}\right)} \sum_{j=1}^{n-1}\left(-\left(\frac{\partial p}{\partial x_{0}} \circ \rho\right)_{x_{j}} \frac{\partial P_{\gamma \lambda} A}{\partial x_{j}}+\left(\frac{\partial p}{\partial x_{j}} \circ \rho\right)_{x_{j}}\right) \lambda d z d \lambda \\
= & -\sum_{j=1}^{n-1} \int_{0}^{\infty} \int_{\mathbb{R}^{n}}\left(\frac{F\left(u_{x_{i}}^{r} \circ \rho\right)}{\left(1+\left|\nabla P_{\gamma \lambda} A\right|^{2}\right)}\right)_{x_{j}}\left(\frac{\partial p}{\partial x_{j}} \circ \rho\right) \lambda d z d \lambda \\
+ & \sum_{j=1}^{n-1} \int_{0}^{\infty} \int_{\mathbb{R}^{n}}\left(\frac{F\left(u_{x_{i}}^{r} \circ \rho\right)}{\left(1+\left|\nabla P_{\gamma \lambda} A\right|^{2}\right)} \frac{\partial P_{\gamma \lambda} A}{\partial x_{j}}\right)_{x_{j}}\left(\frac{\partial p}{\partial x_{0}} \circ \rho\right) \lambda d z d \lambda .
\end{aligned}
$$

Now all the terms in this expansion can be handled using Lemma 1.1, Lemma 2.4 and Lemma 3.3.

Proof of Theorem 3.1. We start by proving (i). Integrating by parts twice in $\lambda$ we have,

$$
\begin{aligned}
I & :=-\frac{1}{2} \int_{\mathbb{R}^{n}}\left(u_{x_{i}}^{r} \circ \rho\right)^{2} d z=\int_{0}^{\infty} \int_{\mathbb{R}^{n}}\left(u_{x_{i}}^{r} \circ \rho\right)\left(u_{x_{i}}^{r} \circ \rho\right)_{\lambda} d z d \lambda \\
& =-\int_{0}^{\infty} \int_{\mathbb{R}^{n}}\left|\left(u_{x_{i}}^{r} \circ \rho\right)_{\lambda}\right|^{2} \lambda d z d \lambda-\int_{0}^{\infty} \int_{\mathbb{R}^{n}}\left(u_{x_{i}}^{r} \circ \rho\right)\left(u_{x_{i}}^{r} \circ \rho\right)_{\lambda \lambda} \lambda d z d \lambda .
\end{aligned}
$$

Using that

$$
\begin{gathered}
\left(u_{x_{i}}^{r} \circ \rho\right)_{\lambda}=\left(u_{x_{i} x_{0}}^{r} \circ \rho\right)\left(1+\frac{\partial P_{\gamma \lambda} A}{\partial \lambda}\right), \\
\left(u_{x_{i}}^{r} \circ \rho\right)_{\lambda \lambda}=\left(u_{x_{i} x_{0}^{2}}^{r} \circ \rho\right)\left(1+\frac{\partial P_{\gamma \lambda} A}{\partial \lambda}\right)^{2}+\left(u_{x_{i} x_{0}}^{r} \circ \rho\right) \frac{\partial^{2} P_{\gamma \lambda} A}{\partial \lambda^{2}}
\end{gathered}
$$

we have that

$$
\begin{aligned}
-\frac{1}{2} \int_{\mathbb{R}^{n}}\left(u_{x_{i}}^{r} \circ \rho\right)^{2} d z & =-\int_{0}^{\infty} \int_{\mathbb{R}^{n}}\left(u_{x_{i} x_{0}}^{r} \circ \rho\right)^{2}\left(1+\frac{\partial P_{\gamma \lambda} A}{\partial \lambda}\right)^{2} \lambda d z d \lambda \\
& -\int_{0}^{\infty} \int_{\mathbb{R}^{n}}^{\infty}\left(u_{x_{i}}^{r} \circ \rho\right)\left(u_{x_{i} x_{0}^{2}}^{r} \circ \rho\right)\left(1+\frac{\partial P_{\gamma \lambda} A}{\partial \lambda}\right)^{2} \lambda d z d \lambda \\
& -\int_{0}^{\infty} \int_{\mathbb{R}^{n}}\left(u_{x_{i}}^{r} \circ \rho\right)\left(u_{x_{i} x_{0}}^{r} \circ \rho\right)\left(\frac{\partial^{2} P_{\gamma \lambda} A}{\partial \lambda^{2}}\right) \lambda d z d \lambda \\
& :=T_{1}+T_{2}+T_{3} .
\end{aligned}
$$


As $u_{t}^{r}=\Delta u^{r}-p_{x_{r}}$ we have that $u_{x_{i} t}^{r}=\Delta u_{x_{i}}^{r}-p_{x_{r} x_{i}}$. Let,

$$
K:=\sum_{k=1}^{n-1}\left[\left(u_{x_{i} x_{0}}^{r} \circ \rho\right)_{x_{k}} \frac{\partial P_{\gamma \lambda} A}{\partial x_{k}}-\left(u_{x_{i} x_{k}}^{r} \circ \rho\right)_{x_{k}}\right] .
$$

Then,

$$
K+u_{x_{i} t}^{r} \circ \rho-\frac{\partial^{2} p}{\partial x_{r} \partial x_{i}} \circ \rho=\left(1+\left|\nabla_{x} P_{\gamma \lambda} A\right|^{2}\right)\left(u_{x_{i} x_{0}^{2}}^{r} \circ \rho\right) .
$$

Substituting this into $T_{2}$ and introducing $E=\left(1+\frac{\partial P_{\gamma \lambda} A}{\partial \lambda}\right)^{2} /\left(1+\left|\nabla_{x} P_{\gamma \lambda} A\right|^{2}\right)$,

$$
\begin{aligned}
T_{2} & =\int_{0}^{\infty} \int_{\mathbb{R}^{n}} \sum_{k=1}^{n-1} \frac{\partial}{\partial x_{k}}\left(E\left(u_{x_{i}}^{r} \circ \rho\right)\right)\left(\left(u_{x_{i} x_{0}}^{r} \circ \rho\right) \frac{\partial P_{\gamma \lambda} A}{\partial x_{k}}-\left(u_{x_{i} x_{k}}^{r} \circ \rho\right)\right) \lambda d z d \lambda \\
& +\int_{0}^{\infty} \int_{\mathbb{R}^{n}} E\left(u_{x_{i}}^{r} \circ \rho\right)\left(\sum_{k=1}^{n-1}\left(u_{x_{i} x_{0}}^{r} \circ \rho\right) \frac{\partial^{2} P_{\gamma \lambda} A}{\partial x_{k}^{2}}\right) \lambda d z d \lambda \\
& -\int_{0}^{\infty} \int_{\mathbb{R}^{n}} E\left(u_{x_{i}}^{r} \circ \rho\right)\left(u_{x_{i}}^{r} \circ \rho\right)_{t} \lambda d z d \lambda \\
& +\int_{0}^{\infty} \int_{\mathbb{R}^{n}} E\left(u_{x_{i}}^{r} \circ \rho\right)\left(u_{x_{i} x_{0}}^{r} \circ \rho\right) \frac{\partial P_{\gamma \lambda} A}{\partial t} \lambda d z d \lambda \\
& -\int_{0}^{\infty} \int_{\mathbb{R}^{n}} E\left(u_{x_{i}}^{r} \circ \rho\right)\left(\frac{\partial^{2} p}{\partial x_{r} \partial x_{i}} \circ \rho\right) \lambda d z d \lambda .
\end{aligned}
$$

Using that

$$
\begin{aligned}
& -\int_{0}^{\infty} \int_{\mathbb{R}^{n}}\left(u_{x_{i} x_{0}}^{r} \circ \rho\right)^{2}\left(1+\frac{\partial P_{\gamma \lambda} A}{\partial \lambda}\right)^{2} \lambda d z d \lambda \\
& +\int_{0}^{\infty} \int_{\mathbb{R}^{n}} \sum_{k=1}^{n-1} \frac{\partial}{\partial x_{k}}\left(E\left(u_{x_{i}}^{r} \circ \rho\right)\right)\left(\left(u_{x_{i} x_{0}}^{r} \circ \rho\right) \frac{\partial P_{\gamma \lambda} A}{\partial x_{k}}-\left(u_{x_{i} x_{k}}^{r} \circ \rho\right)\right) \lambda d z d \lambda \\
& =-\int_{0}^{\infty} \int_{\mathbb{R}^{n}} E \sum_{k=0}^{n-1}\left|u_{x_{i} x_{k}}^{r} \circ \rho\right|^{2} \lambda d z d \lambda \\
& +\int_{0}^{\infty} \int_{\mathbb{R}^{n}} \frac{\partial E}{\partial x_{k}}\left(u_{x_{i}}^{r} \circ \rho\right)\left[\sum_{k=1}^{n-1}\left(u_{x_{i} x_{0}}^{r} \circ \rho\right) \frac{\partial P_{\gamma \lambda} A}{\partial x_{k}}-\left(u_{x_{i} x_{k}}^{r} \circ \rho\right)\right] \lambda d z d \lambda
\end{aligned}
$$

and collecting the expressions we have derived so far,

$$
I=-\int_{0}^{\infty} \int_{\mathbb{R}^{n}} E \sum_{k=1}^{n-1}\left|u_{x_{i} x_{k}}^{r} \circ \rho\right|^{2} \lambda d z d \lambda
$$




$$
\begin{aligned}
& +\int_{0}^{\infty} \int_{\mathbb{R}^{n}} \frac{\partial E}{\partial x_{k}}\left(u_{x_{i}}^{r} \circ \rho\right)\left[\sum_{k=1}^{n-1}\left(u_{x_{i} x_{0}}^{r} \circ \rho\right) \frac{\partial P_{\gamma \lambda} A}{\partial x_{k}}-\left(u_{x_{i} x_{k}}^{r} \circ \rho\right)\right] \lambda d z d \lambda \\
& +\int_{0}^{\infty} \int_{\mathbb{R}^{n}} E\left(u_{x_{i}}^{r} \circ \rho\right)\left(\sum_{k=1}^{n-1}\left(u_{x_{i} x_{0}}^{r} \circ \rho\right) \frac{\partial^{2} P_{\gamma \lambda} A}{\partial x_{k}^{2}}\right) \lambda d z d \lambda \\
& -\int_{0}^{\infty} \int_{\mathbb{R}^{n}} E\left(u_{x_{i}}^{r} \circ \rho\right)\left(u_{x_{i}}^{r} \circ \rho\right)_{t} \lambda d z d \lambda \\
& +\int_{0}^{\infty} \int_{\mathbb{R}^{n}} E\left(u_{x_{i}}^{r} \circ \rho\right)\left(u_{x_{i} x_{0}}^{r} \circ \rho\right) \frac{\partial P_{\gamma \lambda} A}{\partial t} \lambda d z d \lambda \\
& -\int_{0}^{\infty} \int_{\mathbb{R}^{n}} E\left(u_{x_{i}}^{r} \circ \rho\right)\left(\frac{\partial^{2} p}{\partial x_{r} \partial x_{i}} \circ \rho\right) \lambda d z d \lambda \\
& -\int_{0}^{\infty} \int_{\mathbb{R}^{n}}\left(u_{x_{i}}^{r} \circ \rho\right)\left(u_{x_{i} x_{0}}^{r} \circ \rho\right)\left(\frac{\partial^{2} P_{\gamma \lambda} A}{\partial \lambda^{2}}\right) \lambda d z d \lambda .
\end{aligned}
$$

I.e.,

$$
I=S_{1}+S_{2}+S_{3}+S_{4}+S_{5}+S_{6}+S_{7}
$$

by definition. $S_{1}$ is the expression we want to estimate. $S_{2}, S_{3}, S_{5}$ and $S_{7}$ can be handled using Lemma 1.1 and Lemma 2.4. This gives,

$$
\left|S_{2}\right|+\left|S_{3}\right|+\left|S_{5}\right|+\left|S_{7}\right| \leq C\|\vec{f}\|_{2}\left(\sum_{j=0}^{n-1} \int_{0}^{\infty} \int_{\mathbb{R}^{n}}\left|u_{x_{i} x_{j}}^{r} \circ \rho\right|^{2} \lambda d z d \lambda\right)^{1 / 2} .
$$

We therefore need to consider

$$
\begin{aligned}
& S_{4}=-\int_{0}^{\infty} \int_{\mathbb{R}^{n}} E\left(u_{x_{i}}^{r} \circ \rho\right)\left(u_{x_{i}}^{r} \circ \rho\right)_{t} \lambda d z d \lambda . \\
& S_{6}=-\int_{0}^{\infty} \int_{\mathbb{R}^{n}} E\left(u_{x_{i}}^{r} \circ \rho\right)\left(\frac{\partial^{2} p}{\partial x_{r} \partial x_{i}} \circ \rho\right) \lambda d z d \lambda .
\end{aligned}
$$

We first consider $S_{4}$. Integrating by parts w.r.t $\lambda$ we have,

$$
S_{4}=\int_{0}^{\infty} \int_{\mathbb{R}^{n}} E_{\lambda}\left(u_{x_{i}}^{r} \circ \rho\right)\left(u_{x_{i}}^{r} \circ \rho\right)_{t} \lambda^{2} d z d \lambda+\int_{0}^{\infty} \int_{\mathbb{R}^{n}} \frac{E}{2}\left[\left(u_{x_{i}}^{r} \circ \rho\right)^{2}\right]_{t \lambda} \lambda^{2} d z d \lambda .
$$

Integrating by parts w.r.t. to $t$ in the last term we may continue,

$$
\begin{aligned}
& =\int_{0}^{\infty} \int_{\mathbb{R}^{n}} E_{\lambda}\left(u_{x_{i}}^{r} \circ \rho\right)\left(u_{x_{i}}^{r} \circ \rho\right)_{t} \lambda^{2} d z d \lambda-\int_{0}^{\infty} \int_{\mathbb{R}^{n}} E_{t}\left(u_{x_{i}}^{r} \circ \rho\right)\left(u_{x_{i}}^{r} \circ \rho\right)_{\lambda} \lambda^{2} d z d \lambda \\
& :=S_{41}+S_{42} .
\end{aligned}
$$


Calculating $E_{t}$ and using Lemma 1.1, Lemma 2.4 we have

$$
\begin{aligned}
\left|S_{42}\right| \leq & C\|\vec{f}\|_{2}\left(\sum_{r=0}^{n-1} \sum_{j=0}^{n-1} \int_{0}^{\infty} \int_{\mathbb{R}^{n}}\left|u_{x_{i} x_{j}}^{r} \circ \rho\right|^{2} \lambda d z d \lambda\right)^{1 / 2} . \\
S_{41} & =\int_{0}^{\infty} \int_{\mathbb{R}^{n}} E_{\lambda}\left(u_{x_{i}}^{r} \circ \rho\right)\left(u_{x_{i} x_{0}}^{r} \circ \rho\right) \frac{\partial P_{\gamma \lambda} A}{\partial t} \lambda^{2} d z d \lambda \\
& +\int_{0}^{\infty} \int_{\mathbb{R}^{n}} E_{\lambda}\left(u_{x_{i}}^{r} \circ \rho\right)\left(u_{x_{i} t}^{r} \circ \rho\right) \lambda^{2} d z d \lambda \\
& :=S_{411}+S_{412} .
\end{aligned}
$$

Again using Lemma 1.1 and Lemma 2.4 we have,

$$
\left|S_{411}\right| \leq C\|\vec{f}\|_{2}\left(\sum_{r=0}^{n-1} \sum_{j=0}^{n-1} \int_{0}^{\infty} \int_{\mathbb{R}^{n}}\left|u_{x_{i} x_{j}}^{r} \circ \rho\right|^{2} \lambda d z d \lambda\right)^{1 / 2} .
$$

The same estimate is true for $S_{412}$ using the interior regularity estimate in Lemma 2.3 .

We now turn to $S_{6}$. But by Lemma 3.4 ,

$$
\left|S_{6}\right| \leq C\left[\|\vec{f}\|_{2}^{2}+\|\vec{f}\|_{2}\left(\sum_{r=0}^{n-1} \sum_{j=0}^{n-1} \int_{0}^{\infty} \int_{\mathbb{R}^{n}}\left|u_{x_{i} x_{j}}^{r} \circ \rho\right|^{2} \lambda d z d \lambda\right)^{1 / 2}\right] .
$$

Combining our estimates and using Cauchy Schwarz with $\epsilon$ we may conclude the proof of part (i) of the theorem.

Part (ii) is an immediate consequence of part (i), the interior regularity estimate of Lemma 2.3 and the estimate in Lemma 3.3. To prove (iii) we integrate by parts w.r.t $\lambda$,

$$
\begin{aligned}
\int_{0}^{\infty} \int_{\mathbb{R}^{n}}\left|D_{1 / 2}^{t}\left(\vec{u}_{x_{j}} \circ \rho\right)\right|^{2} \lambda d z d \lambda & =2 \int_{0}^{\infty} \int_{\mathbb{R}^{n}} D_{1 / 2}^{t}\left(\vec{u}_{x_{j}} \circ \rho\right) D_{1 / 2}^{t}\left[\left(\vec{u}_{x_{j}} \circ \rho\right)_{\lambda}\right] \lambda^{2} d z d \lambda \\
& =2 c \int_{0}^{\infty} \int_{\mathbb{R}^{n}}\left(\vec{u}_{x_{j}} \circ \rho\right)_{t}\left(\vec{u}_{x_{j}} \circ \rho\right)_{\lambda} \lambda^{2} d z d \lambda .
\end{aligned}
$$

(iii) now follows from $(i),(i i)$, Lemma 1.1 and Lemma 2.4.

4. Smallness of commutators and more Carleson measures. In this section we collect a few estimates of commutators frequently used in the forthcoming sections. By $M_{n}$ we denote the Hardy- Littlewood maximal function in the $n$-variable. We also define a truncated maximal function in the following way. Let $\sigma>0$. Then,

$$
M_{n}^{\sigma}(h(x, \cdot))(t):=\sup _{0<a \leq \sigma^{2}} \frac{1}{2 a} \int_{-a}^{a}\left|h\left(x, z_{n}+t\right)\right| d z_{n} .
$$


The kernel of $H D_{1 / 2}^{t}$ is $k(t)=c \operatorname{sgn}(t)|t|^{-3 / 2}$. Let

$$
k_{1}(t)=k(t) \chi_{\left\{s:|s| \leq R^{2} \lambda^{2}\right\}}(t), \quad k_{2}(t)=k(t) \chi_{\left\{s:|s|>R^{2} \lambda^{2}\right\}}(t),
$$

where $R, \lambda>0$ and let $D_{1}$ and $D_{2}$ be the associated operators. The following lemma is essentially Lemma 5.16 in [19]. We only spell it out in a more general form as it will be used frequently in the forthcoming sections.

Lemma 4.1. Let $R \geq 2$ and let $j \in\{0, \ldots, n-1\}$. Then,

(i) $\left|\left[D_{1}, \frac{\partial P_{\gamma \lambda} A}{\partial x_{j}}\right] g(x, t)\right| \leq c_{\beta} \lambda^{-1} R \gamma^{-2}\left\|\mathbb{D}_{n} A\right\|_{*} M_{n}(g(x, \cdot))(t)$,

(ii) $\left|\left[D_{2}, \frac{\partial P_{\gamma \lambda} A}{\partial x_{j}}\right] g(x, t)\right| \leq c_{\beta} \lambda^{-1} R^{-1} M_{n}(g(x, \cdot))(t)$,

(iii) $\left|\left[D_{1}, \frac{\partial^{2} P_{\gamma \lambda} A}{\partial x_{j} \partial \lambda}\right] g(x, t)\right| \leq c_{\beta} \lambda R M_{n}^{R \lambda}\left(\frac{\partial^{3} P_{\gamma \lambda} A(x, \cdot)}{\partial x_{j} \partial \lambda \partial z_{n}}\right)(t) \phi_{R \lambda} * g(x, \cdot)(t)$,

(iv) $\left|\left[D_{2}, \frac{\partial^{2} P_{\gamma \lambda} A}{\partial x_{j} \partial \lambda}\right] g(x, t)\right| \leq c_{\beta} \lambda^{-1} R^{-1} M_{n}\left(g(x, \cdot) \frac{\partial^{2} P_{\gamma \lambda} A(x, \cdot)}{\partial x_{j} \partial \lambda}\right)(t)$

$$
+c_{\beta} \lambda^{-1} R^{-1} \phi_{R \lambda}^{*} * g(x, \cdot)(t)\left(\frac{\partial^{2} P_{\gamma \lambda} A(x, t)}{\partial x_{j} \partial \lambda}\right)
$$

where $\phi_{R \lambda}(t)=(R \lambda)^{-1}|t|^{-1 / 2} \chi_{\left\{s:|s| \leq(R \lambda)^{2}\right\}}(t), \phi_{R \lambda}^{*}(t)=R \lambda k_{2}(t)$.

Proof.

$$
\left[D_{1}, \frac{\partial P_{\gamma \lambda} A}{\partial x_{j}}\right] g(x, t)=\int_{\left\{s:|s-t| \leq R^{2} \lambda^{2}\right\}} k(t-s)\left(\frac{\partial P_{\gamma \lambda} A(x, s)}{\partial x_{j}}-\frac{\partial P_{\gamma \lambda} A(x, t)}{\partial x_{j}}\right) g(x, s) d s .
$$

Using the mean-value theorem and Lemma 1.1,

$$
\begin{aligned}
& \left|\left[D_{1}, \frac{\partial P_{\gamma \lambda} A}{\partial x_{j}}\right] g(x, t)\right| \\
= & \left|\int_{0}^{1} \int_{\left\{s:|s-t| \leq R^{2} \lambda^{2}\right\}} k(t-s)(t-s) \times \frac{\partial^{2} P_{\gamma \lambda} A}{\partial x_{j} \partial z_{n}}(x, s+\eta(t-s)) g(x, s) d s d \eta\right| \\
\leq & c_{\beta} \gamma^{-2}\left\|\mathbb{D}_{n} A\right\|_{*} \lambda^{-2} \int_{\left\{s:|s-t| \leq R^{2} \lambda^{2}\right\}}|t-s|^{-1 / 2}|g(x, s)| d s \\
\leq & c_{\beta} \gamma^{-2} R\left\|\mathbb{D}_{n} A\right\|_{*} \lambda^{-1} M_{n}(g(x, \cdot))(t) .
\end{aligned}
$$

Also,

$$
\begin{aligned}
& \left|\left[D_{2}, \frac{\partial P_{\gamma \lambda} A}{\partial x_{j}}\right] g(x, t)\right| \\
= & \left|\int_{\left\{s:|s-t|>R^{2} \lambda^{2}\right\}} k(t-s)\left(\frac{\partial P_{\gamma \lambda} A(x, s)}{\partial x_{j}}-\frac{\partial P_{\gamma \lambda} A(x, t)}{\partial x_{j}}\right) g(x, s) d s\right| .
\end{aligned}
$$


Applying part (ii) of Lemma 1.1 we immediately get the estimate stated in part (ii) of the lemma.

$$
\begin{aligned}
& {\left[D_{1}, \frac{\partial^{2} P_{\gamma \lambda} A}{\partial x_{j} \partial \lambda}\right] g(x, t) } \\
= & \int_{\left\{s:|s-t| \leq R^{2} \lambda^{2}\right\}} k(t-s)\left(\frac{\partial^{2} P_{\gamma \lambda} A(x, s)}{\partial x_{j} \partial \lambda}-\frac{\partial^{2} P_{\gamma \lambda} A(x, t)}{\partial x_{j} \partial \lambda}\right) g(x, s) d s .
\end{aligned}
$$

Again using the mean value theorem as well as the truncated maximal function defined above we have the following estimate,

$$
\left|\frac{\partial^{2} P_{\gamma \lambda} A(x, s)}{\partial x_{j} \partial \lambda}-\frac{\partial^{2} P_{\gamma \lambda} A(x, t)}{\partial x_{j} \partial \lambda}\right| \leq c_{\beta}|s-t| M_{n}^{R \lambda}\left(\frac{\partial^{3} P_{\gamma \lambda} A(x, \cdot)}{\partial \lambda \partial x_{j} \partial z_{n}}\right)(t) .
$$

The inequality stated in part (iii) of the lemma is a consequence of this estimate and standard arguments.

$$
\begin{aligned}
& {\left[D_{2}, \frac{\partial^{2} P_{\gamma \lambda} A}{\partial x_{j} \partial \lambda}\right] g(x, t) } \\
= & \int_{\left\{s:|s-t|>R^{2} \lambda^{2}\right\}} k(t-s)\left(\frac{\partial^{2} P_{\gamma \lambda} A(x, s)}{\partial x_{j} \partial \lambda}-\frac{\partial^{2} P_{\gamma \lambda} A(x, t)}{\partial x_{j} \partial \lambda}\right) g(x, s) d s .
\end{aligned}
$$

As in the estimate of $\left[D_{2}, \frac{\partial P_{\gamma \lambda} A}{\partial x_{j}}\right] g(x, t)$,

$$
\begin{aligned}
& \left|\int_{\left\{s:|s-t|>R^{2} \lambda^{2}\right\}} k(t-s) \frac{\partial^{2} P_{\gamma \lambda} A(x, s)}{\partial x_{j} \partial \lambda} g(x, s) d s\right| \\
& \leq c_{\beta} \lambda^{-1} R^{-1} M_{n}\left(g(x, \cdot) \frac{\partial^{2} P_{\gamma \lambda} A(x, \cdot)}{\partial x_{j} \partial \lambda}\right)(t) .
\end{aligned}
$$

Also using Lemma 1.1,

$$
\begin{aligned}
& \left|\int_{\left\{s:|s-t|>R^{2} \lambda^{2}\right\}} k(t-s) \frac{\partial^{2} P_{\gamma \lambda} A(x, t)}{\partial x_{j} \partial \lambda} g(x, s) d s\right| \\
& \leq c_{\beta} \frac{\partial^{2} P_{\gamma \lambda} A(x, t)}{\partial x_{j} \partial \lambda}\left(\phi_{R \lambda}^{*} * g(x, \cdot)\right)(t) \lambda^{-1} R^{-1},
\end{aligned}
$$

where $\phi_{R \lambda}^{*}(t)=R \lambda k_{2}(t)$.

Let in the following $\phi_{R \lambda}(t)=(R \lambda)^{-1}|t|^{-1 / 2} \chi_{\left\{s:|s| \leq(R \lambda)^{2}\right\}}(t)$.

LEMma 4.2. The following measures are Carleson measures on $\mathbb{R}_{+}^{n+1}$ having Carleson norms stated below.

$\begin{aligned} \text { (i) } d \mu_{1}(\lambda, x, t) & =\left[M_{n}^{R \lambda}\left(\frac{\partial^{3} P_{\gamma \lambda} A(x, \cdot)}{\partial x_{j} \partial \lambda \partial z_{n}}\right)(t)\right]^{2} \lambda^{5} d z d \lambda, \\ \text { (ii }) \quad d \mu_{2}(\lambda, x, t) & =\phi_{R \lambda} *\left[M_{n}^{R \lambda}\left(\frac{\partial^{3} P_{\gamma \lambda} A(x, \cdot)}{\partial x_{j} \partial \lambda \partial z_{n}}\right)(t)\right]^{2} \lambda^{5} d z d \lambda,\end{aligned}$ 


$$
\begin{aligned}
& \text { (iii) } d \mu_{3}(\lambda, x, t)=\left|\lambda M_{n}^{\lambda^{2}}\left(\frac{\partial}{\partial z_{n}} P_{\gamma \lambda} A(x, \cdot)\right)(t)\right|^{2} d z \frac{d \lambda}{\lambda}, \\
& \text { (iv) } d \mu_{4}(\lambda, x, t)=\left(\frac{1}{\lambda}\left|\left(I-P_{\gamma \lambda}\right) A(x, t)\right|\right)^{2} d z \frac{d \lambda}{\lambda} . \\
& \text { (i) } \mu_{1}\left((0, r) \times B_{r}(x, t)\right) \leq C_{\beta} \gamma^{-4}\left\|\mathbb{D}_{n} A\right\|_{*}^{2} R^{d} r^{d}, \\
& \text { (ii) } \mu_{2}\left((0, r) \times B_{r}(x, t)\right) \leq C_{\beta} \gamma^{-4}\left\|\mathbb{D}_{n} A\right\|_{*}^{2} R^{d} r^{d}, \\
& \text { (iii) } \mu_{3}\left((0, r) \times B_{r}(x, t)\right) \leq C_{\beta} \gamma^{-2}\left\|\mathbb{D}_{n} A\right\|_{*}^{2}, \\
& \text { (iv) } \mu_{4}\left((0, r) \times B_{r}(x, t)\right) \leq C_{\beta} \gamma^{2}\|A\|_{\text {comm. }} .
\end{aligned}
$$

Proof. The estimates of the measures in part $(i)$ and $(i i)$ can be found in [19, p.394-395]. The estimates in (iii) and (iv) are proved by similar arguments.

LEMMA 4.3. Let $\vec{u}=S \vec{f}$ with $\vec{f} \in L^{2}(\partial \Omega)$. Let $\gamma<1 / 2$ and $\left\|\mathbb{D}_{n} A\right\|_{*} \leq \gamma^{8+d}$. Then the following is true for all tripples $(j, k, r)$,

$$
\int_{0}^{\infty} \int_{\mathbb{R}^{n}}\left|\left[H D_{1 / 2}^{t}, \frac{\partial P_{\gamma \lambda} A}{\partial x_{j}}\right]\left(u_{x_{k}}^{r} \circ \rho\right)\right|^{2} \lambda d z d \lambda \lesssim \gamma^{2}\|\vec{f}\|_{2}^{2} .
$$

Proof. Integrating by parts with respect to $\lambda$ and using Cauchy-Schwarz with $\epsilon$ we have

$$
\begin{aligned}
& \int_{0}^{\infty} \int_{\mathbb{R}^{n}}\left|\left[H D_{1 / 2}^{t}, \frac{\partial P_{\gamma \lambda} A}{\partial x_{j}}\right]\left(u_{x_{k}}^{r} \circ \rho\right)\right|^{2} \lambda d z d \lambda \\
\lesssim & \int_{0}^{\infty} \int_{\mathbb{R}^{n}}\left|\left[H D_{1 / 2}^{t}, \frac{\partial P_{\gamma \lambda} A}{\partial x_{j}}\right]\left(u_{x_{k}}^{r} \circ \rho\right)_{\lambda}\right|^{2} \lambda^{3} d z d \lambda \\
+ & \int_{0}^{\infty} \int_{\mathbb{R}^{n}}\left|\left[H D_{1 / 2}^{t}, \frac{\partial^{2} P_{\gamma \lambda} A}{\partial x_{j} \partial \lambda}\right]\left(u_{x_{k}}^{r} \circ \rho\right)\right|^{2} \lambda^{3} d z d \lambda \\
:= & N_{1}+N_{2} .
\end{aligned}
$$

Using part (i) and (ii) of Lemma 4.1 with $R=\gamma^{-1}$, Lemma 1.1, Lemma 2.4 and Theorem 3.1 we have, $N_{1} \lesssim \gamma^{2}\|\vec{f}\|_{2}^{2}$. Using Lemma 4.1 we have

$$
\begin{aligned}
N_{2} & \lesssim \int_{0}^{\infty} \int_{\mathbb{R}^{n}}\left|R^{-1} M_{n}\left(\left(u_{x_{k}}^{r} \circ \rho\right)\left(\frac{\partial^{2} P_{\gamma \lambda} A(x, \cdot)}{\partial x_{j} \partial \lambda}\right)\right)(t)\right|^{2} \lambda d z d \lambda \\
& +\int_{0}^{\infty} \int_{\mathbb{R}^{n}}\left|R^{-1}\left[\phi_{R \lambda}^{*} *\left(u_{x_{k}}^{r} \circ \rho\right)\right](t)\left(\frac{\partial^{2} P_{\gamma \lambda} A(x, t)}{\partial x_{j} \partial \lambda}\right)\right|^{2} \lambda d z d \lambda \\
& +\int_{0}^{\infty} \int_{\mathbb{R}^{n}}\left|R M_{n}^{R \lambda}\left(\frac{\partial^{3} P_{\gamma \lambda} A(x, \cdot)}{\partial x_{j} \partial \lambda \partial z_{n}}\right)(t) \phi_{R \lambda} *\left(u_{x_{k}}^{r} \circ \rho\right)\right|^{2} \lambda^{5} d z d \lambda .
\end{aligned}
$$


Noting that $\left[\phi_{R \lambda}^{*} *\left(u_{x_{k}}^{r} \circ \rho\right)(x, \cdot)\right](t)$ is non tangentially bounded by $M_{n} N_{*}\left(u_{x_{k}}^{r} \circ \rho\right)$, letting $R=\gamma^{-1}$, using Lemma 2.4, Lemma 1.1 and continuity of the Hardy-Littlewood maximal function we have,

$$
\left|N_{2}\right| \lesssim \gamma^{2}\|\vec{f}\|_{2}^{2}+\int_{0}^{\infty} \int_{\mathbb{R}^{n}}\left|R \phi_{R \lambda} * M_{n}^{R \lambda}\left(\frac{\partial^{3} P_{\gamma \lambda} A(x, \cdot)}{\partial x_{j} \partial \lambda \partial z_{n}}\right)(t)\left(u_{x_{k}}^{r} \circ \rho\right)\right|^{2} \lambda^{5} d z d \lambda .
$$

Choosing $R=\gamma^{-1}$ and using Lemma 4.2 on Carleson measures as well as Lemma 2.4 we may conclude that, $\left|N_{2}\right| \lesssim \gamma^{2}\|\vec{f}\|_{2}^{2}$. This completes the proof of Lemma 4.3.

5. Estimates of the $L^{2}$-norms of certain fractional derivatives of the single layer potential. This section is devoted to the proof of the following theorem.

THEOREM 5.1. Let $\|A\|_{\text {comm }} \leq \beta<\infty$ and $\vec{f} \in L^{2}(\partial \Omega)$. Let $\vec{u}=S \vec{f}$ be the single layer potential associated to the linearized system of Navier-Stokes. Assume that $\gamma \leq 1 / 2$ and $b=\left\|\mathbb{D}_{n} A\right\|_{*} \leq \epsilon_{0}=\gamma^{8+d}$. Then,

$$
\begin{gathered}
\int_{0}^{\infty} \int_{\mathbb{R}^{n}}\left|D_{1 / 4}^{t}\left(\vec{u}_{x_{j}} \circ \rho\right)(\lambda, z)\right|^{2} d z d \lambda \leqq C_{\beta}\|\vec{f}\|_{2}^{2}, \\
\int_{0}^{\infty} \int_{\mathbb{R}^{n}}\left|D_{3 / 4}^{t}(\vec{u} \circ \rho)(\lambda, z)\right|^{2} d z d \lambda \leqq C_{\beta}\left[\gamma\|\vec{f}\|_{2}^{2}+\left\|\frac{\partial \vec{u}}{\partial \nu}\right\|_{2}\left\|S_{b} \vec{f}\right\|_{L_{1,1 / 2}^{2}(\partial \Omega)}\right] .
\end{gathered}
$$

Proof.

$$
\begin{aligned}
& \int_{0}^{\infty} \int_{\mathbb{R}^{n}}\left|D_{1 / 4}^{t}\left(u_{x_{j}}^{r} \circ \rho\right)(\lambda, z)\right|^{2} d z d \lambda=(\text { partial integration in } \lambda) \\
= & -2 \int_{0}^{\infty} \int_{\mathbb{R}^{n}} D_{1 / 4}^{t}\left(u_{x_{j}}^{r} \circ \rho\right) D_{1 / 4}^{t}\left(\left(u_{x_{j}}^{r} \circ \rho\right)_{\lambda}\right) \lambda d z d \lambda \\
= & -2 \int_{0}^{\infty} \int_{\mathbb{R}^{n}} D_{1 / 2}^{t}\left(u_{x_{j}}^{r} \circ \rho\right)\left(u_{x_{j}}^{r} \circ \rho\right)_{\lambda} \lambda d z d \lambda \\
\leq & \left(\int_{0}^{\infty} \int_{\mathbb{R}^{n}}\left|\left(u_{x_{j}}^{r} \circ \rho\right)_{\lambda}\right|^{2} \lambda d z d \lambda\right)^{1 / 2} \times\left(\int_{0}^{\infty} \int_{\mathbb{R}^{n}}\left|D_{1 / 2}^{t}\left(u_{x_{j}}^{r} \circ \rho\right)\right|^{2} \lambda d z d \lambda\right)^{1 / 2} \\
\lesssim & \left(\int_{0}^{\infty} \int_{\mathbb{R}^{n}}\left|\left(u_{x_{j} x_{0}}^{r} \circ \rho\right)\right|^{2} \lambda d z d \lambda\right)^{1 / 2} \times\left(\int_{0}^{\infty} \int_{\mathbb{R}^{n}}\left|D_{1 / 2}^{t}\left(u_{x_{j}}^{r} \circ \rho\right)\right|^{2} \lambda d z d \lambda\right)^{1 / 2} .
\end{aligned}
$$

Using Theorem 3.1 our estimate follows. We now turn to the other estimate in the theorem. Define $\tilde{w}^{r}=H D_{1 / 2}^{t}\left(u^{r} \circ \rho\right)$.

$$
\sum_{r} \int_{0}^{\infty} \int_{\mathbb{R}^{n}}\left|D_{3 / 4}^{t}\left(u^{r} \circ \rho\right)\right|^{2} d z d \lambda=\sum_{r} \int_{0}^{\infty} \int_{\mathbb{R}^{n}} \tilde{w}^{r}\left(u^{r} \circ \rho\right)_{t} d z d \lambda
$$




$$
\begin{aligned}
& =\sum_{r} \int_{0}^{\infty} \int_{\mathbb{R}^{n}} \tilde{w}^{r}\left(u^{r} \circ \rho\right)_{t}\left(1+\frac{\partial P_{\gamma \lambda} A}{\partial \lambda}\right) d z d \lambda-\sum_{r} \int_{0}^{\infty} \int_{\mathbb{R}^{n}} \tilde{w}^{r}\left(u_{t}^{r} \circ \rho\right) \frac{\partial P_{\gamma \lambda} A}{\partial \lambda} d z d \lambda \\
& +\sum_{r} \int_{0}^{\infty} \int_{\mathbb{R}^{n}} \tilde{w}^{r}\left(u_{x_{0}}^{r} \circ \rho\right) \frac{\partial P_{\gamma \lambda} A}{\partial t} \frac{\partial P_{\gamma \lambda} A}{\partial \lambda} d z d \lambda=T_{1}+T_{2}+T_{3} .
\end{aligned}
$$

We first focus on $T_{1}$. Recall the definition of the conormal derivative

$$
\frac{\partial \vec{u}}{\partial \nu}:=(\nabla \vec{u}) N-p N
$$

If $\vec{w}$ is a vector and if $(\vec{u}, p)$ solves the linearized Navier-Stokes then,

$$
\int_{\Omega} \vec{w} \frac{\partial \vec{u}}{\partial t} d X d t=\int_{\Omega} \vec{w} \Delta \vec{u} d X d t-\int_{\Omega} \vec{w} \nabla p d X d t .
$$

But,

$$
\int_{\Omega} w^{r} \Delta u^{r} d X d t=\int_{\partial \Omega} w^{r} \nabla u^{r} N_{t} d \sigma_{t} d t-\int_{\Omega} \nabla w^{r} \nabla u^{r} d X d t .
$$

We now let $\vec{w}=w^{r} e_{r}$ where $w^{r} \circ \rho=\tilde{w}^{r}$ and $e_{r}$ is the unit vector in the $x_{r}$-direction. i.e.,

$$
\begin{aligned}
\int_{\Omega} w^{r} \frac{\partial u^{r}}{\partial t} d X d t & =\int_{\partial \Omega} w^{r} \nabla u^{r} N_{t} d \sigma_{t} d t-\int_{\Omega} \nabla w^{r} \nabla u^{r} d X d t-\int_{\Omega} w^{r} \frac{\partial p}{\partial x_{r}} d X d t \\
& =\int_{\partial \Omega} w^{r}\left(\nabla u^{r} N_{t}-p N_{t}^{r}\right) d \sigma_{t} d t-\int_{\Omega} \nabla w^{r} \nabla u^{r} d X d t \\
& +\int_{\partial \Omega} w^{r} p N_{t}^{r} d \sigma_{t} d t-\int_{\Omega} w^{r} \frac{\partial p}{\partial x_{r}} d X d t .
\end{aligned}
$$

As

$$
\int_{\partial \Omega} w^{r} p N_{t}^{r} d \sigma_{t} d t-\int_{\Omega} w^{r} \frac{\partial p}{\partial x_{r}} d X d t=\int_{\Omega} \frac{\partial w^{r}}{\partial x_{r}} p d X d t
$$

we have

$$
\begin{aligned}
T_{1} & =\sum_{r} \int_{0}^{\infty} \int_{\mathbb{R}^{n}} \tilde{w}^{r}\left(u^{r} \circ \rho\right)_{t}\left(1+\frac{\partial P_{\gamma \lambda} A}{\partial \lambda}\right) d z d \lambda \\
& =\sum_{r}\left[\int_{\partial \Omega} w^{r}\left(\nabla u^{r} N_{t}-p N_{t}^{r}\right) d \sigma_{t} d t-\int_{\Omega} \nabla w^{r} \nabla u^{r} d X d t+\int_{\Omega} \frac{\partial w^{r}}{\partial x_{r}} p d X d t\right] .
\end{aligned}
$$

To estimate $T_{1}$ we therefore have to consider terms of the following forms,

$$
A_{r, j}=-\int_{\Omega} \frac{\partial w^{r}}{\partial x_{j}} \frac{\partial u^{r}}{\partial x_{j}} d X d t \quad B=\sum_{r=0}^{n-1} \int_{\Omega} \frac{\partial w^{r}}{\partial x_{r}} p d X d t .
$$

We postpone the proof of Lemma 5.2 and Lemma 5.3 below in order to complete the proof of Theorem 5.1. Lemma 5.2 is proved at the end of this section. All of Section 6 
is devoted to the proof of Lemma 5.3. As $\operatorname{div} \vec{u}=0$ the term $B$ would be zero if we were studing a time independent cylinder. Now we want to prove that this term is small in an appropriate sense. This turns out to be highly non trivial and for expositure reasons we have devoted a whole section to that term. $\gamma\|\vec{f}\|_{2}^{2}$.

Lemma 5.2. Assume that $\gamma \leq 1 / 2$ and $b=\left\|\mathbb{D}_{n} A\right\|_{*} \leq \epsilon_{0}=\gamma^{8+d}$. Then, $\left|A_{r, j}\right| \lesssim$ $\gamma\|\vec{f}\|_{2}^{2}$.

LEMma 5.3. Assume that $\gamma \leq 1 / 2$ and $b=\left\|\mathbb{D}_{n} A\right\|_{*} \leq \epsilon_{0}=\gamma^{8+d}$. Then, $|B| \lesssim$ Using Lemma 5.2 and Lemma 5.3 we have now proved the following estimate for $T_{1}$,

$$
\left|T_{1}\right| \leq C_{\beta}\left[\gamma\|\vec{f}\|_{2}^{2}+\left\|\frac{\partial \vec{u}}{\partial \nu}\right\|_{2}\left\|S_{b} \vec{f}\right\|_{L_{1,1 / 2}^{2}(\partial \Omega)}\right] .
$$

Recall our main objective, i.e to estimate,

$$
\sum_{r} \int_{0}^{\infty} \int_{\mathbb{R}^{n}}\left|D_{3 / 4}^{t}\left(u^{r} \circ \rho\right)\right|^{2} d z d \lambda:=T_{1}+T_{2}+T_{3} .
$$

$T_{2}$ and $T_{3}$ are left to treat. In the following we use the summation convention. Trivially,

$$
\begin{aligned}
\left|T_{2}\right| & \leq C\left(\int_{0}^{\infty} \int_{\mathbb{R}^{n}}|\tilde{w}|^{2}\left(\frac{\partial P_{\gamma \lambda} A}{\partial \lambda}\right)^{2} \frac{1}{\lambda} d z d \lambda\right)^{1 / 2} \times\left(\int_{0}^{\infty} \int_{\mathbb{R}^{n}}\left|\Delta u^{j} \circ \rho+\frac{\partial p}{\partial x_{j}} \circ \rho\right|^{2} \lambda d z d \lambda\right)^{1 / 2} \\
& \leq C\left(1+\gamma^{-1} b\right)\|\vec{f}\|_{2}^{2} \lesssim \gamma\|\vec{f}\|_{2}^{2}
\end{aligned}
$$

by Lemma 1.1, Lemma 2.4, Lemma 3.3 and Theorem 3.1.

Left is therefore to manipulate $T_{3}$. Integrating by parts w.r.t. $\lambda$ we have that the following formula is valid for $T_{3}$.

$$
\begin{aligned}
T_{3} & =\int_{0}^{\infty} \int_{\mathbb{R}^{n}} \tilde{w}^{r}\left(u_{x_{0}}^{r} \circ \rho\right) \frac{\partial P_{\gamma \lambda} A}{\partial t} d z d \lambda=-\int_{0}^{\infty} \int_{\mathbb{R}^{n}} \tilde{w}_{\lambda}^{r}\left(u_{x_{0}}^{r} \circ \rho\right) \frac{\partial P_{\gamma \lambda} A}{\partial t} \lambda d z d \lambda \\
& -\int_{0}^{\infty} \int_{\mathbb{R}^{n}} \tilde{w}^{r}\left(u_{x_{0}}^{r} \circ \rho\right)_{\lambda} \frac{\partial P_{\gamma \lambda} A}{\partial t} \lambda d z d \lambda-\int_{0}^{\infty} \int_{\mathbb{R}^{n}} \tilde{w}^{r}\left(u_{x_{0}}^{r} \circ \rho\right) \frac{\partial^{2} P_{\gamma \lambda} A}{\partial t \partial \lambda} \lambda d z d \lambda .
\end{aligned}
$$

Using this formula for $T_{3}$ we have

$$
\begin{aligned}
\left|T_{3}\right| \lesssim\left(\int_{0}^{\infty} \int_{\mathbb{R}^{n}}\left|D_{1 / 2}^{t}\left(\left(u^{r} \circ \rho\right)_{\lambda}\right)\right|^{2} \lambda d z d \lambda\right)^{1 / 2}\left(\int_{0}^{\infty} \int_{\mathbb{R}^{n}}\left|\left(u_{x_{0}}^{r} \circ \rho\right)\right|^{2}\left(\frac{\partial P_{\gamma \lambda} A}{\partial t}\right)^{2} \lambda d z d \lambda\right)^{1 / 2} \\
+\left(\int_{0}^{\infty} \int\left|H D_{\mathbb{R}^{n}}^{t}\left(u^{r} \circ \rho\right)\right|^{2}\left(\frac{\partial P_{\gamma \lambda} A}{\partial t}\right)^{2} \lambda d z d \lambda\right)^{1 / 2}\left(\int_{0}^{\infty} \int_{\mathbb{R}^{n}}\left|\left(u_{x_{0} x_{0}}^{r} \circ \rho\right)\right|^{2} \lambda d z d \lambda\right)^{1 / 2} \\
+\left|\int_{0}^{\infty} \int_{\mathbb{R}^{n}} \tilde{w}^{r}\left(u_{x_{0}}^{r} \circ \rho\right) \frac{\partial^{2} P_{\gamma \lambda} A}{\partial t \partial \lambda} \lambda d z d \lambda\right| \\
=M_{1} \cdot N_{1}+M_{2} \cdot N_{2}+A .
\end{aligned}
$$


Applying Lemma 1.1, Lemma 2.4 and Theorem 3.1 we have

$$
\begin{array}{cl}
\left|N_{1}\right| \lesssim \gamma^{-1} b\|\vec{f}\|_{2} & \left|M_{2}\right| \lesssim \gamma^{-1} b\|\vec{f}\|_{2} \\
\left|N_{2}\right| \lesssim\|\vec{f}\|_{2} & \left|M_{2}\right| \lesssim\|\vec{f}\|_{2} .
\end{array}
$$

i.e.,

$$
\left|T_{3}\right| \lesssim\left[\gamma\|\vec{f}\|_{2}^{2}+A\right] .
$$

To estimate $A$ remains. Integrating by parts w.r.t $t$ in $A$ we have

$$
\begin{aligned}
A & =\int_{0}^{\infty} \int_{\mathbb{R}^{n}} H D_{1 / 2}^{t}\left(u^{r} \circ \rho\right)\left(u_{x_{0}}^{r} \circ \rho\right) \frac{\partial P_{\gamma \lambda} A}{\partial t \partial \lambda} \lambda d z d \lambda \\
& =-\int_{0}^{\infty} \int_{\mathbb{R}^{n}}\left[H D_{1 / 2}^{t}\left(u^{r} \circ \rho\right)\right]_{t}\left(u_{x_{0}}^{r} \circ \rho\right) \frac{\partial P_{\gamma \lambda} A}{\partial \lambda} \lambda d z d \lambda \\
& -\int_{0}^{\infty} \int_{\mathbb{R}^{n}} H D_{1 / 2}^{t}\left(u^{r} \circ \rho\right)\left(u_{x_{0}}^{r} \circ \rho\right)_{t} \frac{\partial P_{\gamma \lambda} A}{\partial \lambda} \lambda d z d \lambda \\
& =-\int_{0}^{\infty} \int_{\mathbb{R}^{n}} H D_{1 / 2}^{t}\left[\left(u_{x_{0}}^{r} \circ \rho\right) \frac{\partial P_{\gamma \lambda} A}{\partial t}+\left(u_{t}^{r} \circ \rho\right)\right]\left(u_{x_{0}}^{r} \circ \rho\right) \frac{\partial P_{\gamma \lambda} A}{\partial \lambda} \lambda d z d \lambda \\
& -\int_{0}^{\infty} \int_{\mathbb{R}^{n}} H D_{1 / 2}^{t}\left(u^{r} \circ \rho\right)\left[\left(u_{x_{0} x_{0}}^{r} \circ \rho\right) \frac{\partial P_{\gamma \lambda} A}{\partial t}+\left(u_{x_{0} t}^{r} \circ \rho\right)\right] \frac{\partial P_{\gamma \lambda} A}{\partial \lambda} \lambda d z d \lambda \\
& =\int_{0}^{\infty} \int_{\mathbb{R}^{n}}\left[\left(u_{x_{0}}^{r} \circ \rho\right) \frac{\partial P_{\gamma \lambda} A}{\partial t}+\left(u_{t}^{r} \circ \rho\right)\right] H D_{1 / 2}^{t}\left[\left(u_{x_{0}}^{r} \circ \rho\right) \frac{\partial P_{\gamma \lambda} A}{\partial \lambda}\right] \lambda d z d \lambda \\
& -\int_{0}^{\infty} \int_{\mathbb{R}^{n}} H D_{1 / 2}^{t}\left(u^{r} \circ \rho\right)\left(u_{x_{0} x_{0}}^{r} \circ \rho\right) \frac{\partial P_{\gamma \lambda} A}{\partial t} \frac{\partial P_{\gamma \lambda} A}{\partial \lambda} \lambda d z d \lambda \\
& -\int_{0}^{\infty} \int_{\mathbb{R}^{n}} H D_{1 / 2}^{t}\left(u^{r} \circ \rho\right)\left(u_{x_{0} t}^{r} \circ \rho\right) \frac{\partial P_{\gamma \lambda} A}{\partial \lambda} \lambda d z d \lambda=A_{1}+A_{2}+A_{3} .
\end{aligned}
$$

Using Lemma 2.4, Theorem 3.1 and Lemma 1.1 one may conclude that

$$
\left|A_{2}\right|+\left|A_{3}\right| \leq C_{\beta} \gamma\|\vec{f}\|_{2}^{2} .
$$

$$
\begin{aligned}
A_{1} & =\int_{0}^{\infty} \int_{\mathbb{R}^{n}}\left[\left(u_{x_{0}}^{r} \circ \rho\right) \frac{\partial P_{\gamma \lambda} A}{\partial t}+\left(u_{t}^{r} \circ \rho\right)\right]\left[H D_{1 / 2}^{t}, \frac{\partial P_{\gamma \lambda} A}{\partial \lambda}\right]\left(u_{x_{0}}^{r} \circ \rho\right) \lambda d z d \lambda \\
& +\int_{0}^{\infty} \int_{\mathbb{R}^{n}}\left[\left(u_{x_{0}}^{r} \circ \rho\right) \frac{\partial P_{\gamma \lambda} A}{\partial t}+\left(u_{t}^{r} \circ \rho\right)\right] H D_{1 / 2}^{t}\left[\left(u_{x_{0}}^{r} \circ \rho\right)\right] \frac{\partial P_{\gamma \lambda} A}{\partial \lambda} \lambda d z d \lambda .
\end{aligned}
$$


Applying Lemma 4.3, Lemma 1.1, Lemma 2.4, Theorem 3.1 and Lemma 3.3 in a by now familar way we may conclude that, $\left|A_{1}\right| \leq C_{\beta} \gamma\|f\|_{2}^{2}$. Putting it all together we have proved that $\left|T_{3}\right| \lesssim \gamma\|f\|_{2}^{2}$. This proves Theorem 5.1 modulo Lemma 5.2 and 5.3.

We now turn to the proof of Lemma 5.2. First we will derive an expansion of $A_{r, i}$ from which we will able to handle all terms. Such an expansion was presented in a similar way in [27].

Lemma 5.4. Let $r, j \in\{0, \ldots, n-1\}$. Then

$$
\begin{aligned}
A_{r, j}= & -\delta_{j, 0} \int_{0}^{\infty} \int_{\mathbb{R}^{n}} H D_{1 / 2}^{t}\left(\left(u_{x_{0}}^{r} \circ \rho\right) \frac{\partial P_{\gamma \lambda} A}{\partial \lambda}\right)\left(u_{x_{0}}^{r} \circ \rho\right) d z d \lambda \\
& \left.-\left(1-\delta_{j, 0}\right) \int_{0}^{\infty} \int_{\mathbb{R}^{n}} H D_{1 / 2}^{t}\left[\left(u_{x_{j}}^{r} \circ \rho\right)\right)\right]\left(u_{x_{j}}^{r} \circ \rho\right) \frac{\partial P_{\gamma \lambda} A}{\partial \lambda} d z d \lambda \\
& +\left(1-\delta_{j, 0}\right) \int_{0}^{\infty} \int_{\mathbb{R}^{n}}\left(\left[H D_{1 / 2}^{t}, \frac{\partial P_{\gamma \lambda} A}{\partial x_{j}}\right]\left(u_{x_{0}}^{r} \circ \rho\right)\right)\left(u_{x_{j}}^{r} \circ \rho\right)_{\lambda} \lambda d z d \lambda \\
& +\left(1-\delta_{j, 0}\right) \int_{0}^{\infty} \int_{\mathbb{R}^{n}}\left(\left[H D_{1 / 2}^{t}, \frac{\partial P_{\gamma \lambda} A}{\partial x_{j}}\right]\left(u_{x_{0}}^{r} \circ \rho\right)_{\lambda}\right)\left(u_{x_{j}}^{r} \circ \rho\right) \lambda d z d \lambda \\
& +\left(1-\delta_{j, 0}\right) \int_{0}^{\infty} \int_{\mathbb{R}^{n}} H D_{1 / 2}^{t}\left[\frac{\partial^{2} P_{\gamma \lambda} A}{\partial x_{j} \partial \lambda}\left(u_{x_{j}}^{r} \circ \rho\right)\right]\left(u_{x_{j}}^{r} \circ \rho\right) \lambda d z d \lambda \\
& -\left(1-\delta_{j, 0}\right) \int_{0}^{\infty} \int_{\mathbb{R}^{n}} \frac{\partial^{2} P_{\gamma \lambda} A}{\partial x_{j} \partial \lambda} H D_{1 / 2}^{t}\left[\left(u_{x_{0}}^{r} \circ \rho\right)\right]\left(u_{x_{j}}^{r} \circ \rho\right) \lambda d z d \lambda \\
& -\left(1-\delta_{j, 0}^{\infty}\right) \int_{0}^{\infty} \int_{\mathbb{R}^{n}}^{\infty} H D_{1 / 2}^{t}\left[\left(u_{x_{0}}^{r} \circ \rho\right) \frac{\partial P_{\gamma \lambda} A}{\partial x_{j}}\right]\left(u_{x_{j}}^{r} \circ \rho\right) \frac{\partial P_{\gamma \lambda} A}{\partial \lambda} d z d \lambda . \\
& -\left(1-\delta_{j, 0}\right) \int_{\mathbb{R}^{n}}^{t} H D_{1 / 2}^{t}\left[\left(u_{x_{j}}^{r} \circ \rho\right) \frac{\partial P_{\gamma \lambda} A}{\partial x_{j}}\right]\left(u_{x_{0}}^{r} \circ \rho\right) \frac{\partial P_{\gamma \lambda} A}{\partial \lambda} d z d \lambda \\
& -(1 \lambda
\end{aligned}
$$

Proof of Lemma 5.4.

$$
\begin{aligned}
A_{r, 0} & =-\int_{\Omega} \frac{\partial w^{r}}{\partial x_{0}} \frac{\partial u^{r}}{\partial x_{0}} d X d t=-\int_{\partial \Omega} \tilde{w}_{\lambda}^{r}\left(u_{x_{0}}^{r} \circ \rho\right)\left(1+\frac{\partial P_{\gamma \lambda} A}{\partial \lambda}\right) d z d \lambda \\
& =-\int_{0}^{\infty} \int_{\mathbb{R}^{n}} H D_{1 / 2}^{t}\left(\left(u_{x_{0}}^{r} \circ \rho\right)_{\lambda}\right)\left(u_{x_{0}}^{r} \circ \rho\right)\left(1+\frac{\partial P_{\gamma \lambda} A}{\partial \lambda}\right) d z d \lambda \\
& =-\int_{0}^{\infty} \int_{\mathbb{R}^{n}}\left(H D_{1 / 2}^{t}\left(u_{x_{0}}^{r} \circ \rho\right)\right)\left(u_{x_{0}}^{r} \circ \rho\right)\left(1+\frac{\partial P_{\gamma \lambda} A}{\partial \lambda}\right) d z d \lambda
\end{aligned}
$$




$$
\begin{aligned}
& -\int_{0}^{\infty} \int_{\mathbb{R}^{n}} H D_{1 / 2}^{t}\left(\left(u_{x_{0}}^{r} \circ \rho\right) \frac{\partial P_{\gamma \lambda} A}{\partial \lambda}\right)\left(u_{x_{0}}^{r} \circ \rho\right)\left(1+\frac{\partial P_{\gamma \lambda} A}{\partial \lambda}\right) d z d \lambda \\
& =-\int_{0}^{\infty} \int_{\mathbb{R}^{n}} H D_{1 / 2}^{t}\left(\left(u_{x_{0}}^{r} \circ \rho\right) \frac{\partial P_{\gamma \lambda} A}{\partial \lambda}\right)\left(u_{x_{0}}^{r} \circ \rho\right) d z d \lambda,
\end{aligned}
$$

by anti-symmetry. Let $j \neq 0$. Then

$$
A_{r, j}=-\int_{0}^{\infty} \int_{\mathbb{R}^{n}}\left(w_{x_{j}} \circ \rho\right)\left(u_{x_{j}}^{r} \circ \rho\right)\left(1+\frac{\partial P_{\gamma \lambda} A}{\partial \lambda}\right) d z d \lambda
$$

If we define $D=1+\frac{\partial P_{\gamma \lambda} A}{\partial \lambda}$ then,

$$
\begin{aligned}
w_{x_{j}}^{r} \circ \rho & =\tilde{w}_{x_{j}}^{r}-\tilde{w}_{\lambda}^{r} \frac{\partial P_{\gamma \lambda} A}{\partial x_{j}} \frac{1}{1+\frac{\partial P_{\gamma \lambda} A}{\partial \lambda}} \\
& =\tilde{w}_{x_{j}}^{r}-\tilde{w}_{\lambda}^{r} \frac{\partial P_{\gamma \lambda} A}{\partial \lambda} \frac{1}{D} .
\end{aligned}
$$

But

$$
\begin{aligned}
& \tilde{w}_{x_{j}}^{r}=H D_{1 / 2}^{t}\left(u_{x_{j}}^{r} \circ \rho\right)+H D_{1 / 2}^{t}\left[\left(u_{x_{0}}^{r} \circ \rho\right) \frac{\partial P_{\gamma \lambda} A}{\partial x_{j}}\right] \\
& \tilde{w}_{\lambda}^{r}=H D_{1 / 2}^{t}\left[\left(u_{x_{0}}^{r} \circ \rho\right) D\right] .
\end{aligned}
$$

I.e.

$$
\begin{aligned}
\tilde{w}_{x_{j}}^{r} & =H D_{1 / 2}^{t}\left(u_{x_{j}}^{r} \circ \rho\right)+H D_{1 / 2}^{t}\left[\left(u_{x_{0}}^{r} \circ \rho\right) \frac{\partial P_{\gamma \lambda} A}{\partial x_{j}}\right] \\
& -\frac{1}{D} \frac{\partial P_{\gamma \lambda} A}{\partial x_{j}} H D_{1 / 2}^{t}\left[\left(u_{x_{0}}^{r} \circ \rho\right) D\right] .
\end{aligned}
$$

I.e.

$$
\begin{aligned}
A_{r, j}= & -\int_{0}^{\infty} \int_{\mathbb{R}^{n}} H D_{1 / 2}^{t}\left[\left(u_{x_{j}}^{r} \circ \rho\right)\right]\left(u_{x_{j}}^{r} \circ \rho\right) D d z d \lambda \\
& -\int_{0}^{\infty} \int_{\mathbb{R}^{n}} H D_{1 / 2}^{t}\left[\left(u_{x_{0}}^{r} \circ \rho\right) \frac{\partial P_{\gamma \lambda} A}{\partial x_{j}}\right]\left(u_{x_{j}}^{r} \circ \rho\right) d z d \lambda \\
& +\int_{0}^{\infty} \int_{\mathbb{R}^{n}} \frac{\partial P_{\gamma \lambda} A}{\partial x_{j}} H D_{1 / 2}^{t}\left[\left(u_{x_{0}}^{r} \circ \rho\right) D\right]\left(u_{x_{j}}^{r} \circ \rho\right) d z d \lambda \\
= & -\int_{0}^{\infty} \int_{\mathbb{R}^{n}} H D_{1 / 2}^{t}\left[\left(u_{x_{j}}^{r} \circ \rho\right)\right]\left(u_{x_{j}}^{r} \circ \rho\right) d z d \lambda \\
& -\int_{0}^{\infty} \int_{\mathbb{R}^{n}} H D_{1 / 2}^{t}\left[\left(u_{x_{j}}^{r} \circ \rho\right)\right]\left(u_{x_{j}}^{r} \circ \rho\right) \frac{\partial P_{\gamma \lambda} A}{\partial \lambda} d z d \lambda
\end{aligned}
$$




$$
\begin{aligned}
& -\int_{0}^{\infty} \int_{\mathbb{R}^{n}}\left(\left[H D_{1 / 2}^{t}, \frac{\partial P_{\gamma \lambda} A}{\partial x_{j}}\right]\left(u_{x_{0}}^{r} \circ \rho\right)\right)\left(u_{x_{j}}^{r} \circ \rho\right) d z d \lambda \\
& \left.+\int_{0}^{\infty} \int_{\mathbb{R}^{n}} H D_{1 / 2}^{t}\left[\left(u_{x_{0}}^{r} \circ \rho\right)\right] \frac{\partial P_{\gamma \lambda} A}{\partial \lambda}\right]\left(u_{x_{j}}^{r} \circ \rho\right) \frac{\partial P_{\gamma \lambda} A}{\partial x_{j}} d z d \lambda .
\end{aligned}
$$

Integrating by parts in $\lambda$ we have

$$
\begin{aligned}
& -\int_{0}^{\infty} \int_{\mathbb{R}^{n}}\left(\left[H D_{1 / 2}^{t}, \frac{\partial P_{\gamma \lambda} A}{\partial x_{j}}\right]\left(u_{x_{0}}^{r} \circ \rho\right)\right)\left(u_{x_{j}}^{r} \circ \rho\right) d z d \lambda \\
& =\int_{0}^{\infty} \int_{\mathbb{R}^{n}}\left(\left[H D_{1 / 2}^{t}, \frac{\partial P_{\gamma \lambda} A}{\partial x_{j}}\right]\left(u_{x_{0}}^{r} \circ \rho\right)\right)\left(u_{x_{j}}^{r} \circ \rho\right)_{\lambda} \lambda d z d \lambda \\
& +\int_{0}^{\infty} \int_{\mathbb{R}^{n}}\left(\left[H D_{1 / 2}^{t}, \frac{\partial P_{\gamma \lambda} A}{\partial x_{j}}\right]\left(u_{x_{0}}^{r} \circ \rho\right)_{\lambda}\right)\left(u_{x_{j}}^{r} \circ \rho\right) \lambda d z d \lambda \\
& +\int_{0}^{\infty} \int_{\mathbb{R}^{n}}\left(\left[H D_{1 / 2}^{t}, \frac{\partial^{2} P_{\gamma \lambda} A}{\partial x_{j} \partial \lambda}\right]\left(u_{x_{0}}^{r} \circ \rho\right)\right)\left(u_{x_{j}}^{r} \circ \rho\right) \lambda d z d \lambda
\end{aligned}
$$

Note that by the anti-symmetry of $H D_{1 / 2}^{t}$ we have

$$
\begin{aligned}
& \int_{0}^{\infty} \int_{\mathbb{R}^{n}} H D_{1 / 2}^{t}\left[\left(u_{x_{j}}^{r} \circ \rho\right)\right]\left(u_{x_{j}}^{r} \circ \rho\right) d z d \lambda=0, \\
& \int_{0}^{\infty} \int_{\mathbb{R}^{n}} H D_{1 / 2}^{t}\left[\left(u_{x_{0}}^{r} \circ \rho\right) \frac{\partial P_{\gamma \lambda} A}{\partial \lambda}\right]\left(u_{x_{j}}^{r} \circ \rho\right) \frac{\partial P_{\gamma \lambda} A}{\partial x_{j}} d z d \lambda \\
= & \int_{0}^{\infty} \int_{\mathbb{R}^{n}} H D_{1 / 2}^{t}\left[\left(u_{x_{j}}^{r} \circ \rho\right) \frac{\partial P_{\gamma \lambda} A}{\partial x_{j}}\right]\left(u_{x_{0}}^{r} \circ \rho\right) \frac{\partial P_{\gamma \lambda} A}{\partial \lambda} d z d \lambda .
\end{aligned}
$$

Collecting we get the formula stated in the lemma.

Proof of Lemma 5.2. We intend to estimate $\left|A_{r, j}\right|$. Using Lemma 5.4 we have that $A_{r, j}=\sum_{k=1}^{8} M_{k}$ where $M_{k}$ are the expressions stated in the lemma. Using the antisymmetry of $H D_{1 / 2}^{t}$ and the self-adjointness of the commutator all of these terms can be expressed on one of the following forms for relevant indicies $r, j, m$ and $k$,

$$
\begin{aligned}
& \left.N_{1}=\int_{0}^{\infty} \int_{\mathbb{R}^{n}} H D_{1 / 2}^{t}\left[\left(u_{x_{k}}^{r} \circ \rho\right)\right)\right]\left(u_{x_{j}}^{r} \circ \rho\right) \frac{\partial P_{\gamma \lambda} A}{\partial \lambda} d z d \lambda \\
& N_{2}=\int_{0}^{\infty} \int_{\mathbb{R}^{n}}\left(\left[H D_{1 / 2}^{t}, \frac{\partial P_{\gamma \lambda} A}{\partial x_{j}}\right]\left(u_{x_{k}}^{r} \circ \rho\right)\right)\left(u_{x_{m}}^{r} \circ \rho\right)_{\lambda} \lambda d z d \lambda
\end{aligned}
$$




$$
\begin{aligned}
& N_{3}=\int_{0}^{\infty} \int_{\mathbb{R}^{n}} \frac{\partial^{2} P_{\gamma \lambda} A}{\partial x_{j} \partial \lambda}\left(u_{x_{k}}^{r} \circ \rho\right) H D_{1 / 2}^{t}\left(u_{x_{m}}^{r} \circ \rho\right) \lambda d z d \lambda \\
& N_{4}=\int_{0}^{\infty} \int_{\mathbb{R}^{n}} H D_{1 / 2}^{t}\left[\left(u_{x_{k}}^{r} \circ \rho\right) \frac{\partial P_{\gamma \lambda} A}{\partial x_{j}}\right]\left(u_{x_{m}}^{r} \circ \rho\right) \frac{\partial P_{\gamma \lambda} A}{\partial \lambda} d z d \lambda .
\end{aligned}
$$

Applying Lemma 1.1 and Theorem 3.1 we have,

$$
\begin{aligned}
\left|N_{1}\right| & \leq\left(\int_{0}^{\infty} \int_{\mathbb{R}^{n}}\left|H D_{1 / 2}^{t}\left[\left(u_{x_{k}}^{r} \circ \rho\right)\right]\right|^{2} \lambda d z d \lambda\right)^{1 / 2}\left(\int_{0}^{\infty} \int_{\mathbb{R}^{n}}\left|\left(u_{x_{j}}^{r} \circ \rho\right)\right|^{2}\left(\frac{\partial P_{\gamma \lambda} A}{\partial \lambda}\right)^{2} \frac{1}{\lambda} d z d \lambda\right)^{1 / 2} \\
& \lesssim \gamma\|\vec{f}\|_{2}^{2} .
\end{aligned}
$$

Using Theorem 3.1 and Lemma 4.3 we get,

$$
\left|N_{2}\right| \lesssim\|\vec{f}\|_{2}\left(\int_{0}^{\infty} \int_{\mathbb{R}^{n}}\left|\left[H D_{1 / 2}^{t}, \frac{\partial P_{\gamma \lambda} A}{\partial x_{j}}\right]\left(u_{x_{k}}^{r} \circ \rho\right)\right|^{2} \lambda d z d \lambda\right)^{1 / 2} \lesssim \gamma\|\vec{f}\|_{2}^{2}
$$

Integrating by parts w.r.t. $x_{j}$ in $N_{3}$ we have

$$
\begin{aligned}
N_{3}= & -\int_{0}^{\infty} \int_{\mathbb{R}^{n}} \frac{\partial P_{\gamma \lambda} A}{\partial \lambda}\left(u_{x_{k}}^{r} \circ \rho\right)_{x_{j}} H D_{1 / 2}^{t}\left(u_{x_{m}}^{r} \circ \rho\right) \lambda d z d \lambda \\
& -\int_{0}^{\infty} \int_{\mathbb{R}^{n}} \frac{\partial P_{\gamma \lambda} A}{\partial \lambda}\left(u_{x_{k}}^{r} \circ \rho\right) H D_{1 / 2}^{t}\left[\left(u_{x_{m}}^{r} \circ \rho\right)_{x_{j}}\right] \lambda d z d \lambda \\
= & -\int_{0}^{\infty} \int_{\mathbb{R}^{n}} \frac{\partial P_{\gamma \lambda} A}{\partial \lambda}\left(u_{x_{k}}^{r} \circ \rho\right)_{x_{j}} H D_{1 / 2}^{t}\left(u_{x_{m}}^{r} \circ \rho\right) \lambda d z d \lambda \\
& +\int_{0}^{\infty} \int_{\mathbb{R}^{n}}^{\infty}\left[H D_{1 / 2}^{t}, \frac{\partial P_{\gamma \lambda} A}{\partial \lambda}\right]\left(u_{x_{k}}^{r} \circ \rho\right)\left(u_{x_{m}}^{r} \circ \rho\right)_{x_{j}} \lambda d z d \lambda \\
& +\int_{0}^{\infty} \int_{\mathbb{R}^{n}} \frac{\partial P_{\gamma \lambda} A}{\partial \lambda}\left[H D_{1 / 2}^{t}\left(u_{x_{k}}^{r} \circ \rho\right)\right]\left(u_{x_{m}}^{r} \circ \rho\right)_{x_{j}} \lambda d z d \lambda .
\end{aligned}
$$

As above $\left|N_{3}\right| \lesssim \gamma\|\vec{f}\|_{2}^{2}$. The same estimate is true for $\left|N_{4}\right|$. This is proven by similar manipulations.

6. The estimate of a difficult term involving the pressure. This section is devoted to the proof of Lemma 5.3 in the previous section. I.e., define $\tilde{w}^{r}=$ $H D_{1 / 2}^{t}\left(u^{r} \circ \rho\right)$ where $u^{r}$ is component $r$ of the single layer potential associated to the system of linearized Navier-Stokes. We want to estimate

$$
B:=\sum_{r=0}^{n-1} \int_{\Omega} \frac{\partial w^{r}}{\partial x_{r}} p d X d t
$$


where $w^{r} \circ \rho=\tilde{w}^{r}$. As $\operatorname{div} \vec{u}=0$ the sum under consideration would be zero if we were considering a time-independent cylinder. We will prove that in our situation $B$ is small in the sense that if $\gamma<1 / 2$ is small enough and if $b=\left\|\mathbb{D}_{n} A\right\|_{*} \leq \gamma^{8+d}$ then $|B| \lesssim \gamma\|\vec{f}\|_{2}^{2}$. Changing coordinates,

$$
B=\sum_{r=0}^{n-1} \int_{0}^{\infty} \int_{\mathbb{R}^{n}}\left(\frac{\partial w^{r}}{\partial x_{r}} \circ \rho\right)(p \circ \rho)\left(1+\frac{\partial P_{\gamma \lambda} A}{\partial \lambda}\right) d z d \lambda
$$

Recall that if we define $D=1+\frac{\partial P_{\gamma \lambda} A}{\partial \lambda}$ then for $r \neq 0$,

$w_{x_{r}}^{r} \circ \rho=\left(w^{r} \circ \rho\right)_{x_{r}}-\left(w^{r} \circ \rho\right)_{\lambda} \frac{\partial P_{\gamma \lambda} A}{\partial x_{r}} \frac{1}{1+\frac{\partial P_{\gamma \lambda} A}{\partial \lambda}}=\left(w^{r} \circ \rho\right)_{x_{r}}-\left(w^{r} \circ \rho\right)_{\lambda} \frac{\partial P_{\gamma \lambda} A}{\partial x_{r}} \frac{1}{D}$,

$w_{x_{0}}^{0} \circ \rho=\left(w^{0} \circ \rho\right)_{\lambda} \frac{1}{D}$.

Using this we may write,

$$
\begin{aligned}
B & =\int_{0}^{\infty} \int_{\mathbb{R}^{n}}\left(w^{0} \circ \rho\right)_{\lambda}(p \circ \rho) d z d \lambda \\
& +\sum_{r=1}^{n-1}\left[\int_{0}^{\infty} \int_{\mathbb{R}^{n}}\left(w^{r} \circ \rho\right)_{x_{r}}(p \circ \rho)\left(1+\frac{\partial P_{\gamma \lambda} A}{\partial \lambda}\right) d z d \lambda\right. \\
& \left.-\int_{0}^{\infty} \int_{\mathbb{R}^{n}}\left(w^{r} \circ \rho\right)_{\lambda}(p \circ \rho) \frac{\partial P_{\gamma \lambda} A}{\partial x_{r}} d z d \lambda\right]
\end{aligned}
$$

Using that $\operatorname{div} \vec{u}=0$ we can derive the following equality,

$$
H D_{1 / 2}^{t}\left[\left(u^{0} \circ \rho\right)_{\lambda}\right]+\sum_{r=1}^{n-1} H D_{1 / 2}^{t}\left[\left(u^{r} \circ \rho\right)_{x_{r}} D-\left(u^{r} \circ \rho\right)_{\lambda} \frac{\partial P_{\gamma \lambda} A}{\partial x_{r}}\right]=0 .
$$

Combining these identities with the facts that,

$$
\begin{aligned}
H D_{1 / 2}^{t}\left[\left(u^{0} \circ \rho\right)_{\lambda}\right] & =H D_{1 / 2}^{t}\left[\left(u_{x_{0}}^{0} \circ \rho\right) D\right], \\
H D_{1 / 2}^{t}\left[\left(u^{r} \circ \rho\right)_{x_{r}}\right] & =H D_{1 / 2}^{t}\left[\left(u_{x_{r}}^{r} \circ \rho\right)+\left(u_{x_{0}}^{r} \circ \rho\right) \frac{\partial P_{\gamma \lambda} A}{\partial x_{r}}\right],
\end{aligned}
$$

we have the following equality,

$$
\begin{aligned}
& H D_{1 / 2}^{t}\left[\left(u^{0} \circ \rho\right)_{\lambda}\right]+\sum_{r=1}^{n-1} H D_{1 / 2}^{t}\left[\left(u^{r} \circ \rho\right)_{x_{r}}\right] \\
= & -\left[\sum_{r=1}^{n-1} H D_{1 / 2}^{t}\left[\left(u_{x_{r}}^{r} \circ \rho\right) \frac{\partial P_{\gamma \lambda} A}{\partial \lambda}-\left(u_{x_{0}}^{r} \circ \rho\right) \frac{\partial P_{\gamma \lambda} A}{\partial x_{r}}\right]\right] .
\end{aligned}
$$

Continuing the derivation started above we may therefore conclude, after some ma- 
nipulations,

$$
\begin{aligned}
B & =\sum_{r=1}^{n-1}\left[\int_{0}^{\infty} \int_{\mathbb{R}^{n}}\left[H D_{1 / 2}^{t}, \frac{\partial P_{\gamma \lambda} A}{\partial x_{r}}\right]\left(u^{r} \circ \rho\right)_{\lambda}(p \circ \rho) d z d \lambda\right. \\
& \left.-\int_{0}^{\infty} \int_{\mathbb{R}^{n}}\left[H D_{1 / 2}^{t}, \frac{\partial P_{\gamma \lambda} A}{\partial \lambda}\right]\left(u^{r} \circ \rho\right)_{x_{r}}(p \circ \rho) d z d \lambda\right] .
\end{aligned}
$$

We have to prove that this term is small. For simplicity we just consider the term

$$
T:=-\int_{0}^{\infty} \int_{\mathbb{R}^{n}}^{\infty}\left[H D_{1 / 2}^{t}, \frac{\partial P_{\gamma \lambda} A}{\partial x_{r}}\right]\left(u^{r} \circ \rho\right)_{\lambda}(p \circ \rho) d z d \lambda
$$

the other one being treated similarly. Integrating by parts w.r.t $\lambda$ we have,

$$
\begin{aligned}
T & =\int_{0}^{\infty} \int_{\mathbb{R}^{n}}\left[H D_{1 / 2}^{t}, \frac{\partial P_{\gamma \lambda} A}{\partial x_{r}}\right]\left(u^{r} \circ \rho\right)_{\lambda}(p \circ \rho)_{\lambda} \lambda d z d \lambda \\
& +\int_{0}^{\infty} \int_{\mathbb{R}^{n}}\left[H D_{1 / 2}^{t}, \frac{\partial P_{\gamma \lambda} A}{\partial x_{r}}\right]\left(u^{r} \circ \rho\right)_{\lambda \lambda}(p \circ \rho) \lambda d z d \lambda \\
& +\int_{0}^{\infty} \int_{\mathbb{R}^{n}}\left[H D_{1 / 2}^{t}, \frac{\partial^{2} P_{\gamma \lambda} A}{\partial x_{r} \partial \lambda}\right]\left(u^{r} \circ \rho\right)_{\lambda}(p \circ \rho) \lambda d z d \lambda \\
& =: T_{1}+T_{2}+T_{3} .
\end{aligned}
$$

$T_{1}$ can be easily handled using Cauchy-Schwarz giving,

$$
\left|T_{1}\right| \leq C\left(\int_{0}^{\infty} \int\left|\left[H D_{\mathbb{R}^{n}}^{t} \mid \frac{\partial P_{\gamma \lambda} A}{\partial x_{r}}\right]\left(u^{r} \circ \rho\right)_{\lambda}\right|^{2} \lambda d z d \lambda\right)^{1 / 2} \times\left(\int_{0}^{\infty} \int_{\mathbb{R}^{n}}|\nabla p \circ \rho|^{2} \lambda d z d \lambda\right)^{1 / 2} .
$$

Using Lemma 3.3 and Lemma 4.3 and $b \leq \gamma^{8+d}$ we may conclude that $\left|T_{1}\right| \lesssim \gamma\|\vec{f}\|_{2}^{2}$.

We now have to estimate $T_{2}$ and $T_{3}$. The problem here is that we have no good estimates for the parabolic non-tangential maximal function of $p \circ \rho$. Let in the following $P_{\lambda}$, as usual, be a smooth parabolic approximation to the identity. We now decompose the pressure in the following way,

$$
(p \circ \rho)(\lambda, x, t)=P_{\lambda}(p \circ \rho)+\left(I-P_{\lambda}\right)(p \circ \rho) .
$$

The following is true (the proof is given at the end of the section),

LEMMA 6.1.

$$
\left\|N_{*}\left(P_{\lambda}(p \circ \rho)\right)(x, t)\right\|_{L^{2}\left(\mathbb{R}^{n}, d x d t\right)} \leq c\|\vec{f}\|_{L^{2}(\partial \Omega)} .
$$

Using the decomposition of the pressure we can write,

$$
T_{2}=\int_{0}^{\infty} \int_{\mathbb{R}^{n}}\left[H D_{1 / 2}^{t}, \frac{\partial P_{\gamma \lambda} A}{\partial x_{r}}\right]\left(u^{r} \circ \rho\right)_{\lambda \lambda} P_{\lambda}(p \circ \rho) \lambda d z d \lambda
$$




$$
\begin{aligned}
& +\int_{0}^{\infty} \int_{\mathbb{R}^{n}}\left[H D_{1 / 2}^{t}, \frac{\partial P_{\gamma \lambda} A}{\partial x_{r}}\right]\left(u^{r} \circ \rho\right)_{\lambda \lambda}\left(I-P_{\lambda}\right)(p \circ \rho) \lambda d z d \lambda \\
& =T_{21}+T_{22}
\end{aligned}
$$

Using Theorem 3.1, Lemma 1.1 and Lemma 2.4 we have

$$
\left|T_{21}\right| \lesssim\|\vec{f}\|_{2}\left(\int_{0}^{\infty} \int\left|\left[H D_{1 / 2}^{t}, \frac{\partial P_{\gamma \lambda} A}{\partial x_{r}}\right] P_{\lambda}(p \circ \rho)\right|^{2} \lambda d z d \lambda\right)^{1 / 2}
$$

Integrating by parts w.r.t $x_{r}$ in $T_{3}$ we have

$$
\begin{aligned}
T_{3} & =-\int_{0}^{\infty} \int_{\mathbb{R}^{n}}\left[H D_{1 / 2}^{t}, \frac{\partial P_{\gamma \lambda} A}{\partial \lambda}\right]\left(u^{r} \circ \rho\right)_{x_{r} \lambda}(p \circ \rho) \lambda d z d \lambda \\
& -\int_{0}^{\infty} \int_{\mathbb{R}^{n}}\left[H D_{1 / 2}^{t}, \frac{\partial P_{\gamma \lambda} A}{\partial \lambda}\right]\left(u^{r} \circ \rho\right)_{\lambda}(p \circ \rho)_{x_{r}} \lambda d z d \lambda \\
& =T_{31}+T_{32} .
\end{aligned}
$$

Using Lemma 3.3 and slightly adjusting the argument in the proof of Lemma 4.3, $\left|T_{32}\right| \lesssim \gamma\|\vec{f}\|_{2}^{2}$. Decomposing the pressure in $T_{31}$ we have

$$
\begin{aligned}
T_{31} & =-\int_{0}^{\infty} \int_{\mathbb{R}^{n}}\left[H D_{1 / 2}^{t}, \frac{\partial P_{\gamma \lambda} A}{\partial \lambda}\right]\left(u^{r} \circ \rho\right)_{x_{r} \lambda} P_{\lambda}(p \circ \rho) \lambda d z d \lambda \\
& -\int_{0}^{\infty} \int_{\mathbb{R}^{n}}\left[H D_{1 / 2}^{t}, \frac{\partial P_{\gamma \lambda} A}{\partial \lambda}\right]\left(u^{r} \circ \rho\right)_{x_{r} \lambda}\left(I-P_{\lambda}\right)(p \circ \rho) \lambda d z d \lambda \\
& =T_{311}+T_{312} .
\end{aligned}
$$

As above

$$
\left|T_{311}\right| \lesssim\|\vec{f}\|_{2}\left(\int_{0}^{\infty} \int_{\mathbb{R}^{n}}\left|\left[H D_{1 / 2}^{t}, \frac{\partial P_{\gamma \lambda} A}{\partial \lambda}\right] P_{\lambda}(p \circ \rho)\right|^{2} \lambda d z d \lambda\right)^{1 / 2}
$$

We may therefore conclude that

$$
\begin{aligned}
\left|T_{2}\right|+\left|T_{3}\right| & \lesssim \gamma\|\vec{f}\|_{2}^{2}+\|\vec{f}\|_{2}\left(\int_{0}^{\infty} \int\left|\left[H D_{\mathbb{R}^{n}}^{t} \mid 2, \frac{\partial P_{\gamma \lambda} A}{\partial x_{r}}\right] P_{\lambda}(p \circ \rho)\right|^{2} \lambda d z d \lambda\right)^{1 / 2} \\
& +\|\vec{f}\|_{2}\left(\int_{0}^{\infty} \int_{\mathbb{R}^{n}}\left|\left[H D_{1 / 2}^{t}, \frac{\partial P_{\gamma \lambda} A}{\partial \lambda}\right] P_{\lambda}(p \circ \rho)\right|^{2} \lambda d z d \lambda\right)^{1 / 2} \\
& +\left|\int_{0}^{\infty} \int_{\mathbb{R}^{n}}\left[H D_{1 / 2}^{t}, \frac{\partial P_{\gamma \lambda} A}{\partial x_{r}}\right]\left(u^{r} \circ \rho\right)_{\lambda \lambda}\left(I-P_{\lambda}\right)(p \circ \rho) \lambda d z d \lambda\right|
\end{aligned}
$$




$$
+\left|\int_{0}^{\infty} \int_{\mathbb{R}^{n}}\left[H D_{1 / 2}^{t}, \frac{\partial P_{\gamma \lambda} A}{\partial \lambda}\right]\left(u^{r} \circ \rho\right)_{x_{r} \lambda}\left(I-P_{\lambda}\right)(p \circ \rho) \lambda d z d \lambda\right| .
$$

We first treat the term

$$
A:=\int_{0}^{\infty} \int_{\mathbb{R}^{n}}\left|\left[H D_{1 / 2}^{t}, \frac{\partial P_{\gamma \lambda} A}{\partial x_{r}}\right] P_{\lambda}(p \circ \rho)\right|^{2} \lambda d z d \lambda .
$$

The term containing $\frac{\partial P_{\gamma \lambda} A}{\partial \lambda}$ instead of $\frac{\partial P_{\gamma \lambda} A}{\partial x_{r}}$ is treated in the same way. Integrating the expression in A w.r.t $\lambda$ we have after some simple manipulations that,

$$
\begin{aligned}
A & \lesssim \int_{0}^{\infty} \int_{\mathbb{R}^{n}}\left|\left[H D_{1 / 2}^{t}, \frac{\partial P_{\gamma \lambda} A}{\partial x_{r}}\right] \frac{\partial}{\partial \lambda}\left(P_{\lambda}(p \circ \rho)\right)\right|^{2} \lambda^{3} d z d \lambda \\
& +\int_{0}^{\infty} \int_{\mathbb{R}^{n}}\left|\left[H D_{1 / 2}^{t}, \frac{\partial P_{\gamma \lambda} A}{\partial x_{r} \partial \lambda}\right] P_{\lambda}(p \circ \rho)\right|^{2} \lambda^{3} d z d \lambda \\
& :=A_{1}+A_{2} .
\end{aligned}
$$

Using Lemma 4.1 with $R=\gamma^{-1}$ and $\left\|\mathbb{D}_{n} A\right\|_{*} \leq \gamma^{8+d}$ we have that

$$
\begin{aligned}
A_{1} & \lesssim\left(R^{-2}+R^{2} \gamma^{-4}\left\|\mathbb{D}_{n} A\right\|_{*}^{2}\right) \int_{0}^{\infty} \int_{\mathbb{R}^{n}}\left|\frac{\partial}{\partial \lambda}\left(P_{\lambda}(p \circ \rho)\right)\right|^{2} \lambda d z d \lambda \\
& \lesssim \gamma \int_{0}^{\infty} \int_{\mathbb{R}^{n}}\left|\frac{\partial}{\partial \lambda}\left(P_{\lambda}(p \circ \rho)\right)\right|^{2} \lambda d z d \lambda .
\end{aligned}
$$

From now on we let $P_{\lambda}$ have the following product structure, $P_{\lambda}(x, t)=P_{\lambda}^{(1)}(x) P_{\lambda}^{(2)}(t)$ where $P_{\lambda}^{(1)}(x)=\lambda^{-n+1} \phi(|x| / \lambda)$ and $P_{\lambda}^{(2)}(t)=\lambda^{-2} \phi(|t| / \lambda)$. Here $\phi \in C_{0}^{\infty}(-1,1)$ and $\int \phi=1$. Then

$$
\frac{\partial P_{\lambda}}{\partial \lambda}=\frac{1}{\lambda}\left(Q_{\lambda}^{(1)}\right)^{2} P_{\lambda}^{(2)}+\frac{1}{\lambda}\left(Q_{\lambda}^{(2)}\right)^{2} P_{\lambda}^{(1)},
$$

where $Q_{\lambda}^{(i)}$ is an approximation to the zero operator. I.e., $Q_{\lambda}^{(i)}$ denotes the operator of convolution with a generic kernel of the form $Q_{\lambda}^{(i)}(\cdot)=\lambda^{d_{i}} Q^{(i)}\left(\cdot / \lambda^{\alpha_{i}}\right)$ where $Q^{(i)} \in$ $C_{0}^{\infty}\left(\mathbb{R}^{n_{i}}\right)$ and $\int_{\mathbb{R}^{n_{i}}} Q^{(i)}(z) d z=0$. Here $d_{1}=n-1, \alpha_{1}=n-1, n_{1}=n-1, d_{2}=2$, $\alpha_{2}=2, n_{2}=1$.

Now

$$
\frac{\partial}{\partial \lambda}\left(P_{\lambda}(p \circ \rho)(\lambda, x, t)\right)=P_{\lambda}\left((p \circ \rho)_{\lambda}\right)(\lambda, x, t)+\left(\frac{1}{\lambda}\left(Q_{\lambda}^{(1)}\right)^{2} P_{\lambda}^{(2)}+\frac{1}{\lambda}\left(Q_{\lambda}^{(2)}\right)^{2} P_{\lambda}^{(1)}\right)(p \circ \rho) .
$$

Using this,

$$
\int_{0}^{\infty} \int_{\mathbb{R}^{n}}\left|\frac{\partial}{\partial \lambda}\left(P_{\lambda}(p \circ \rho)\right)\right|^{2} \lambda d z d \lambda \lesssim \int_{0}^{\infty} \int_{\mathbb{R}^{n}}\left|P_{\lambda}\left((p \circ \rho)_{\lambda}\right)\right|^{2} \lambda d z d \lambda
$$




$$
\begin{aligned}
& +\int_{0}^{\infty} \int_{\mathbb{R}^{n}}\left|\left(Q_{\lambda}^{(1)}\right)^{2} P_{\lambda}^{(2)}(p \circ \rho)\right|^{2} \frac{1}{\lambda} d z d \lambda+\int_{0}^{\infty} \int_{\mathbb{R}^{n}}\left|\left(Q_{\lambda}^{(2)}\right)^{2} P_{\lambda}^{(1)}(p \circ \rho)\right|^{2} \frac{1}{\lambda} d z d \lambda \\
& =A_{11}+A_{12}+A_{13} .
\end{aligned}
$$

$A_{11}$ is treated by using the square function estimate of Lemma $3.3, A_{11} \lesssim\|\vec{f}\|_{2}^{2}$. We now estimate $A_{12}$. Let $\psi_{\lambda}(x)$ be the convolution kernel of $\left(Q_{\lambda}^{(1)}\right)^{2}$. Then $\int \psi_{\lambda}=0$ and

$$
\begin{aligned}
& \left|\left(Q_{\lambda}^{(1)}\right)^{2}(p \circ \rho)(\lambda, x, t)\right| \\
= & \left|\int_{\mathbb{R}^{n-1}} \psi_{\lambda}(x-y)[p \circ \rho(\lambda, y, t)-p \circ \rho(\lambda, x, t)] d y\right| \\
= & \left|\int_{0}^{1} \int_{\mathbb{R}^{n-1}} \psi_{\lambda}(x-y)(x-y) \cdot\left[\nabla_{x}(p \circ \rho)\right](\lambda, y+\theta(x-y), t) d y d \theta\right| \\
= & \left|\int_{0}^{1} \int_{\mathbb{R}^{n-1}} \psi_{\lambda}(h) h \cdot\left[\nabla_{x}(p \circ \rho)\right](\lambda, x-(1-\theta) h, t) d h d \theta\right| \\
\leq & c \lambda M^{(1)}\left(\left|\nabla_{x}(p \circ \rho)\right|\right)(\lambda, x, t),
\end{aligned}
$$

where $M^{(1)}$ is the Hardy-Littlewood maximal function in the $x$-variable. Using square function estimates for the pressure, i.e. Lemma 3.3, as well as continuity of maximal functions we may conclude that $A_{12} \lesssim\|\vec{f}\|_{2}^{2}$. Using an argument identical to the one in the proof of Lemma 6.2 below one may prove that

$$
A_{13} \lesssim \int_{0}^{\infty} \int_{\mathbb{R}^{n}}\left|\left(Q_{\lambda}^{(2)}\right)^{2}(p \circ \rho)(\lambda, x, t)\right|^{2} \frac{1}{\lambda} d x d t d \lambda \leq C_{\beta}\|\vec{f}\|_{2}^{2} .
$$

Put together, $A_{1} \lesssim \gamma\|\vec{f}\|_{2}^{2}$. Using Lemma 4.1 we have

$$
\begin{aligned}
\left|A_{2}\right| & \lesssim \int_{0}^{\infty} \int_{\mathbb{R}^{n}}\left|R^{-1} M_{n}\left(P_{\lambda}(p \circ \rho)\left(\frac{\partial^{2} P_{\gamma \lambda} A(x, \cdot)}{\partial x_{j} \partial \lambda}\right)\right)(t)\right|^{2} \lambda d z d \lambda \\
& +\int_{0}^{\infty} \int_{\mathbb{R}^{n}}\left|R^{-1}\left(\phi_{R \lambda}^{*} *\left(P_{\lambda}(p \circ \rho)\right)\right)(x, \cdot)\left(\frac{\partial^{2} P_{\gamma \lambda} A(x, t)}{\partial x_{j} \partial \lambda}\right)\right|^{2} \lambda d z d \lambda \\
& +\int_{0}^{\infty} \int_{\mathbb{R}^{n}}\left|R M_{n}^{R \lambda}\left(\frac{\partial^{3} P_{\gamma \lambda} A(x, \cdot)}{\partial x_{j} \partial \lambda \partial z_{n}}\right)(t) \phi_{R \lambda} *\left(P_{\lambda}(p \circ \rho)\right)(x, t)\right|^{2} \lambda^{5} d z d \lambda .
\end{aligned}
$$

Noting that $\phi_{R \lambda}^{*} *\left(P_{\lambda}(p \circ \rho)\right)$ is nontangentially bounded by $M_{n} N_{*}\left(P_{\lambda}(p \circ \rho)\right)$, letting $R=\gamma^{-1}$, using Lemma 6.1, Lemma 1.1 and continuity of the Hardy-Littlewood maximal function we have,

$$
\left|A_{2}\right| \lesssim \gamma\|\vec{f}\|_{2}^{2}+\int_{0}^{\infty} \int_{\mathbb{R}^{n}}\left|R \phi_{R \lambda} * M_{n}^{R \lambda}\left(\frac{\partial^{3} P_{\gamma \lambda} A(x, \cdot)}{\partial x_{j} \partial \lambda \partial z_{n}}\right)(t)\left(P_{\lambda}(p \circ \rho)\right)(x, \cdot)(t)\right|^{2} \lambda^{5} d z d \lambda .
$$


Choosing $R=\gamma^{-1}$ and using Lemma 4.2 on Carleson measures as well as Lemma 6.1 we may conclude that, $\left|A_{2}\right| \lesssim \gamma\|\vec{f}\|_{2}^{2}$.

We may therefore conclude that $|T| \lesssim \gamma\|\vec{f}\|_{2}^{2}+|B|+|C|$, where

$$
\begin{aligned}
B & =\int_{0}^{\infty} \int_{\mathbb{R}^{n}}\left[H D_{1 / 2}^{t}, \frac{\partial P_{\gamma \lambda} A}{\partial x_{r}}\right]\left(u^{r} \circ \rho\right)_{\lambda \lambda}\left(I-P_{\lambda}\right)(p \circ \rho) \lambda d z d \lambda \\
C & =\int_{0}^{\infty} \int_{\mathbb{R}^{n}}^{\infty}\left[H D_{1 / 2}^{t}, \frac{\partial P_{\gamma \lambda} A}{\partial \lambda}\right]\left(u^{r} \circ \rho\right)_{x_{r} \lambda}\left(I-P_{\lambda}\right)(p \circ \rho) \lambda d z d \lambda .
\end{aligned}
$$

We just treat $B$ as $C$ is treated analogously. Using the notation introduced above,

$$
-\left(I-P_{\lambda}\right)(p \circ \rho)=\int_{0}^{\lambda} P_{\nu}^{(2)}\left(Q_{\nu}^{(1)}\right)^{2}(p \circ \rho)(\lambda) \frac{d \nu}{\nu}+\int_{0}^{\lambda} P_{\nu}^{(1)}\left(Q_{\nu}^{(2)}\right)^{2}(p \circ \rho)(\lambda) \frac{d \nu}{\nu} .
$$

In estimating the expression in $\mathrm{B}$ we therefore have to consider

$$
\begin{aligned}
B_{1} & =\int_{0}^{\infty} \int_{\mathbb{R}^{n}} \int_{0}^{\lambda}\left[H D_{1 / 2}^{t}, \frac{\partial P_{\gamma \lambda} A}{\partial x_{r}}\right]\left(u^{r} \circ \rho\right)_{\lambda \lambda} P_{\nu}^{(2)}\left(Q_{\nu}^{(1)}\right)^{2}(p \circ \rho) \lambda \frac{d \nu}{\nu} d z d \lambda, \\
B_{2} & =\int_{0}^{\infty} \int_{\mathbb{R}^{n}} \int_{0}^{\lambda}\left[H D_{1 / 2}^{t}, \frac{\partial P_{\gamma \lambda} A}{\partial x_{r}}\right]\left(u^{r} \circ \rho\right)_{\lambda \lambda} P_{\nu}^{(1)}\left(Q_{\nu}^{(2)}\right)^{2}(p \circ \rho) \lambda \frac{d \nu}{\nu} d z d \lambda .
\end{aligned}
$$

Note that as above,

$$
\begin{aligned}
\left|\left(Q_{\nu}^{(1)}\right)^{2}(p \circ \rho)(\lambda, x, t)\right| & =\left|\int \psi_{\nu}(x-y)[p \circ \rho(\lambda, y, t)-p \circ \rho(\lambda, x, t)] d y\right| \\
& \leq c \nu M^{(1)}\left(\left|\nabla_{x}(p \circ \rho)\right|\right)(\lambda, x, t),
\end{aligned}
$$

where $M^{(1)}$ is the Hardy-Littlewood maximal function in the $x$-variable. Using this observation, the selfadjointness of the commutator and Lemma 4.1 we have

$$
\begin{aligned}
& \left|B_{1}\right|=\left|\int_{0}^{\infty} \int_{\mathbb{R}^{n}} \int_{0}^{\lambda}\left(u^{r} \circ \rho\right)_{\lambda \lambda}\left[H D_{1 / 2}^{t}, \frac{\partial P_{\gamma \lambda} A}{\partial x_{r}}\right]\left[P_{\nu}^{(2)}\left(Q_{\nu}^{(1)}\right)^{2}(p \circ \rho)\right] \lambda \frac{d \nu}{\nu} d z d \lambda\right| \\
& \leq c\left(R^{-2}+R^{2} \gamma^{-4}\left\|\mathbb{D}_{n} A\right\|_{*}\right) \int_{0}^{\infty} \int_{\mathbb{R}^{n}} \int_{0}^{\lambda}\left|\left(u^{r} \circ \rho\right)_{\lambda \lambda}\right|\left|M^{(2)}\left(P_{\nu}^{(2)}\left(Q_{\nu}^{(1)}\right)^{2}(p \circ \rho)\right)\right| \frac{d \nu}{\nu} d z d \lambda \\
& \leq c\left(R^{-2}+R^{2} \gamma^{-4}\left\|\mathbb{D}_{n} A\right\|_{*}\right) \int_{0}^{\infty} \int_{\mathbb{R}^{n}} \int_{0}^{\lambda}\left|\left(u^{r} \circ \rho\right)_{\lambda \lambda}\right| M^{(2)} M^{(2)} M^{(1)}\left(\left|\nabla_{x}(p \circ \rho)\right|\right)(\lambda, x, t) d \nu d z d \lambda \\
& \leq c\left(R^{-2}+R^{2} \gamma^{-4}\left\|\mathbb{D}_{n} A\right\|_{*}\right)\|\vec{f}\|_{2}^{2} \lesssim \gamma\|\vec{f}\|_{2}^{2}
\end{aligned}
$$

by the continuity of the maximal functions, the square function estimates of Theorem 3.1 and Lemma 3.3 and the choice $R=\gamma^{-1}$. Left is to treat the term $B_{2}$. Let in the 
following $\epsilon=1 / 2$.

$$
\begin{aligned}
\left|B_{2}\right| & \leq\left(\int_{0}^{\infty} \int_{\mathbb{R}^{n}} \int_{0}^{\lambda}\left|Q_{\nu}^{(2)}\left[H D_{1 / 2}^{t}, \frac{\partial P_{\gamma \lambda} A}{\partial x_{r}}\right]\left(u^{r} \circ \rho\right)_{\lambda \lambda}\right|^{2}\left(\frac{\nu}{\lambda}\right)^{-\epsilon} d z \frac{d \nu}{\nu} \lambda^{3} d \lambda\right)^{1 / 2} \\
& \times\left(\int_{0}^{\infty} \int_{\mathbb{R}^{n}} \int_{0}^{\lambda}\left|P_{\nu}^{(1)} Q_{\nu}^{(2)}(p \circ \rho)\right|^{2}\left(\frac{\nu}{\lambda}\right)^{\epsilon} d z \frac{d \nu}{\nu} \frac{d \lambda}{\lambda}\right)^{1 / 2} .
\end{aligned}
$$

Note that,

$$
\begin{aligned}
\int_{0}^{\infty} \int_{\mathbb{R}^{n}} \int_{0}^{\lambda}\left|P_{\nu}^{(1)} Q_{\nu}^{(2)}(p \circ \rho)\right|^{2}\left(\frac{\nu}{\lambda}\right)^{\epsilon} d z \frac{d \nu}{\nu} \frac{d \lambda}{\lambda} & \leq C \int_{0}^{\infty} \int_{\mathbb{R}^{n}} \int_{0}^{\lambda}\left|M^{(1)}\left(Q_{\nu}^{(2)}(p \circ \rho)\right)\right|^{2}\left(\frac{\nu}{\lambda}\right)^{\epsilon} d z \frac{d \nu}{\nu} \frac{d \lambda}{\lambda} \\
& \leq C \int_{0}^{\infty} \int_{\mathbb{R}^{n}}^{\lambda} \int_{0}^{\lambda}\left|Q_{\nu}^{(2)}(p \circ \rho)\right|^{2}\left(\frac{\nu}{\lambda}\right)^{\epsilon} d z \frac{d \nu}{\nu} \frac{d \lambda}{\lambda} .
\end{aligned}
$$

I.e., what we have to do is to estimate the terms,

$$
\begin{aligned}
B_{21} & :=\int_{0}^{\infty} \int_{\mathbb{R}^{n}} \int_{0}^{\lambda}\left|Q_{\nu}^{(2)}(p \circ \rho)\right|^{2}\left(\frac{\nu}{\lambda}\right)^{\epsilon} \frac{d \nu}{\nu} d z \frac{d \lambda}{\lambda}=\int_{0}^{\infty} \int_{\mathbb{R}^{n}}^{\infty} \int_{\nu}^{\infty}\left|Q_{\nu}^{(2)}(p \circ \rho)\right|^{2}\left(\frac{\nu}{\lambda}\right)^{\epsilon} \frac{d \lambda}{\lambda} d z \frac{d \nu}{\nu} \\
B_{22} & :=\int_{0}^{\infty} \int_{\mathbb{R}^{n}} \int_{0}^{\lambda}\left|Q_{\nu}^{(2)}\left[H D_{1 / 2}^{t}, \frac{\partial P_{\gamma \lambda} A}{\partial x_{r}}\right]\left(u^{r} \circ \rho\right)_{\lambda \lambda}\right|^{2}\left(\frac{\nu}{\lambda}\right)^{-\epsilon} d z \frac{d \nu}{\nu} \lambda^{3} d \lambda .
\end{aligned}
$$

We first consider $B_{21}$. Integrating by parts w.r.t $\lambda$ we have $(\epsilon=1 / 2)$,

$$
\begin{aligned}
B_{21} & =2 \int_{0}^{\infty} \int_{\mathbb{R}^{n}}\left|Q_{\nu}^{(2)}(p \circ \rho)(\nu, x, t)\right|^{2} d z \frac{d \nu}{\nu} \\
& +4 \int_{0}^{\infty} \int_{\mathbb{R}^{n}} \int_{0}^{\lambda} Q_{\nu}^{(2)}(p \circ \rho) Q_{\nu}^{(2)}(p \circ \rho)_{\lambda}\left(\frac{\nu}{\lambda}\right)^{1 / 2} d \lambda d z \frac{d \nu}{\nu}=B_{211}+B_{212} .
\end{aligned}
$$

Using Cauchy-Schwarz we have,

$$
\begin{aligned}
\left|B_{212}\right| & \leq\left(\int_{0}^{\infty} \int_{\mathbb{R}^{n}} \int_{0}^{\lambda}\left|Q_{\nu}^{(2)}(p \circ \rho)\right|^{2}\left(\frac{\nu}{\lambda}\right)^{1 / 2} \frac{d \nu}{\nu} d z \frac{d \lambda}{\lambda}\right)^{1 / 2} \\
& \times\left(\int_{0}^{\infty} \int_{\mathbb{R}^{n}} \int_{0}^{\lambda}\left|Q_{\nu}^{(2)}(p \circ \rho)_{\lambda}\right|^{2}\left(\frac{\nu}{\lambda}\right)^{1 / 2} \frac{d \nu}{\nu} d z \lambda d \lambda\right)^{1 / 2} .
\end{aligned}
$$

Using Cauchy-Schwarz with $\epsilon$ and standard arguments we have,

$$
\left|B_{21}\right| \lesssim\left|B_{211}\right|+\int_{0}^{\infty} \int_{\mathbb{R}^{n}} \int_{0}^{\lambda}\left|Q_{\nu}^{(2)}(p \circ \rho)_{\lambda}\right|^{2}\left(\frac{\nu}{\lambda}\right)^{1 / 2} \frac{d \nu}{\nu} d z \lambda d \lambda
$$




$$
\lesssim\left|B_{211}\right|+\int_{0}^{\infty} \int_{\mathbb{R}^{n}}\left|(p \circ \rho)_{\lambda}\right|^{2} \lambda d \lambda d z \lesssim\left|B_{211}\right|+\|\vec{f}\|_{2}^{2}
$$

Lemma 6.2 .

$$
\int_{0}^{\infty} \int_{\mathbb{R}^{n}}\left|Q_{\nu}^{(2)}(p \circ \rho)(\nu, x, t)\right|^{2} d z \frac{d \nu}{\nu} \leq C_{\beta}\|\vec{f}\|_{2}^{2}
$$

Using lemma 6.2 we may conclude that $\left|B_{21}\right| \lesssim\|\vec{f}\|_{2}^{2}$. Left is therefore to estimate

$$
B_{22}:=\int_{0}^{\infty} \int_{\mathbb{R}^{n}} \int_{0}^{\lambda}\left|Q_{\nu}^{(2)}\left[H D_{1 / 2}^{t}, \frac{\partial P_{\gamma \lambda} A}{\partial x_{r}}\right]\left(u^{r} \circ \rho\right)_{\lambda \lambda}\right|^{2}\left(\frac{\nu}{\lambda}\right)^{-\epsilon} d z \frac{d \nu}{\nu} \lambda^{3} d \lambda .
$$

But the relevant estimate of this term is stated in the following lemma.

LEMmA 6.3. Let $R \geq 2$. Then there exist integers $m_{1}$ and $m_{2}$ such that,

$$
\left|B_{22}\right| \leq C_{\beta}\left(R^{-2}+R^{m_{1}} \gamma^{-m_{2}}\right)\|\vec{f}\|_{2}^{2} .
$$

By an appropriate choice of $R$ we may therefore conclude that,

$$
|B| \leq\left|B_{1}\right|+\left|B_{2}\right| \lesssim \gamma\|\vec{f}\|_{2}+\left(B_{21} \times B_{22}\right)^{1 / 2} \lesssim \gamma\|\vec{f}\|_{2}+\|\vec{f}\|_{2}^{1 / 2} \times \gamma\|\vec{f}\|_{2}^{1 / 2} \lesssim \gamma\|\vec{f}\|_{2}^{2} .
$$

The proof of Lemma 5.3 of the previous section is therefore complete modulo Lemma 6.1-6.3.

We now end the section with the proof of Lemma 6.1, 6.2 and 6.3.

Proof of Lemma 6.1. Recall that

$$
p(X, t)=\int_{\partial \Omega_{t}} \nabla \Gamma(X-Q) \vec{f}(Q, t) d \sigma_{t}(Q),
$$

where $\Gamma$ in this context is the fundamental solution of Laplace operator. Let

$$
g(\lambda, x, t)=(p \circ \rho)(\lambda, x, t)=\int_{\mathbb{R}^{n-1}} K(\lambda, x, y, t) \vec{g}(y, t) d y
$$

where $\|\vec{g}\|_{L^{2}\left(\mathbb{R}^{n}, d x d t\right)} \sim\|\vec{f}\|_{L^{2}(\partial \Omega)}$ and $K(\lambda, x, y, t)=\nabla \Gamma\left(\lambda+P_{\gamma \lambda} A(x, t)-A(y, t), x-y\right)$. We may without loss of generality assume that $\vec{g}$ is non negative. Let $\phi_{\lambda}$ be a smooth function of $(y, s)$. Let $\lambda>0$ and $(x, t) \in B_{a \lambda}\left(x_{0}, t_{0}\right)$. Assume that $\phi_{\lambda}=1$ on $B_{c a \lambda}\left(x_{0}, t_{0}\right)$ and $\phi_{\lambda}=0$ on the complement of $B_{2 c a \lambda}\left(x_{0}, t_{0}\right)$ for some constant $c>>1$. We decompose,

$$
\vec{g}(y, s)=\phi_{\lambda} \vec{g}(y, s)+\left(1-\phi_{\lambda}\right) \vec{g}(y, s)=\vec{g}_{1}(y, s)+\vec{g}_{2}(y, s) .
$$

Then $g(\lambda, x, t)=g_{1}(\lambda, x, t)+g_{2}(\lambda, x, t)$ and one easily derives that

$$
\left|P_{\lambda} g_{1}(\lambda, x, t)\right| \leq \sum_{r} c \lambda^{-d} \int_{B_{c a \lambda}\left(x_{0}, t_{0}\right)}\left|g_{1}^{r}(y, s)\right| d y d s \leq \sum_{r} c M\left(g^{r}\right)\left(x_{0}, t_{0}\right),
$$


where $M$ is a parabolic Hardy-Littlewood maximal function. We now consider $P_{\lambda} g_{2}(\lambda, x, t)$.

$$
\begin{aligned}
& P_{\lambda} g_{2}(\lambda, x, t) \\
= & \int_{\mathbb{R}^{n}} \int_{\mathbb{R}^{n-1}} \lambda^{-d} P\left(\frac{x-z}{\lambda}, \frac{t-s}{\lambda^{2}}\right)\left(K(\lambda, z, y, s)-K\left(0, x_{0}, y, s\right)\right) \vec{g}_{2}(y, s) d y d z d s \\
+ & \int_{\mathbb{R}^{n}} \int_{\mathbb{R}^{n-1}} \lambda^{-d} P\left(\frac{x_{0}-z}{\lambda}, \frac{t_{0}-s}{\lambda^{2}}\right) K\left(0, x_{0}, y, s\right) \vec{g}_{2}(y, s) d y d z d s=M_{1}+M_{2} .
\end{aligned}
$$

Obviously,

$$
\left|M_{2}\right| \leq c M^{(2)}\left(g_{2}\left(0, x_{0}, \cdot\right)\right)\left(t_{0}\right),
$$

where again $M^{(2)}$ is a Hardy-Littlewood maximal function with respect to $t$ with good continuity in $L^{p}$. We now focus on $M_{1}$.

$$
\begin{gathered}
K(\lambda, z, y, s)-K\left(0, x_{0}, y, s\right) \\
=K(\lambda, z, y, s)-K\left(\lambda, x_{0}, y, s\right)+K\left(\lambda, x_{0}, y, s\right)-K\left(0, x_{0}, y, s\right) . \\
\left|K(\lambda, z, y, s)-K\left(\lambda, x_{0}, y, s\right)\right| \\
=\left|\nabla \Gamma\left(\lambda+P_{\gamma \lambda} A(z, s)-A(y, s), z-y\right)-\nabla \Gamma\left(\lambda+P_{\gamma \lambda} A\left(x_{0}, s\right)-A(y, s), x_{0}-y\right)\right| \\
\leq c\left|x_{0}-y\right|^{-n}\left[\left|P_{\gamma \lambda} A(z, s)-P_{\gamma \lambda} A\left(x_{0}, s\right)\right|+\left|z-x_{0}\right|\right] \leq c \lambda\left|x_{0}-y\right|^{-n} .
\end{gathered}
$$

Analogously

$$
\left|K\left(\lambda, x_{0}, y, s\right)-K\left(0, x_{0}, y, s\right)\right| \leq c \lambda\left|x_{0}-y\right|^{-n} .
$$

I.e,

$$
\begin{aligned}
& \left|P_{\lambda} g_{2}(\lambda, x, t)\right| \leq c M^{(2)}\left(g_{2}\left(0, x_{0}, \cdot\right)\right)\left(t_{0}\right) \\
+ & \sum_{r} \sum_{j=0}^{\infty} \lambda^{-d} \lambda \int_{2^{j} \lambda \leq\left|x_{0}-y\right|<2^{j+1} \lambda} \int_{\left|x_{0}-z\right|<c \lambda\left|t_{0}-s\right|<c \lambda^{2}} \int_{\left|x_{0}-y\right|^{n}} \frac{g_{2}^{r}(y, s)}{\mid x_{0}} d s d y d z \\
\leq & c M^{(2)}\left(g_{2}\left(0, x_{0}, \cdot\right)\right)\left(t_{0}\right)+\sum_{r} c M^{(1)}\left(M^{(2)}\left(g_{2}^{r}(-, \cdot)\left(t_{0}\right)\right)\left(x_{0}\right) .\right.
\end{aligned}
$$

Again, the desired continuity follows by continuity of maximal functions and as $p \circ$ $\rho(0, x, t)$ is continous in $L^{2}\left(\mathbb{R}^{n}, d x d t\right)$.

Proof of Lemma 6.2. Recall once again that

$$
p(X, t)=\int_{\partial \Omega_{t}} \nabla \Gamma(X-Q, t) \vec{f}(Q, t) d \sigma_{t}(Q),
$$

where $\Gamma$ in this context is the fundamental solution of Laplace operator and

$$
(p \circ \rho)(\nu, x, t)=\int_{\mathbb{R}^{n-1}} K(\nu, x, y, t) \vec{g}(y, t) d y,
$$


where $\|\vec{g}\|_{L^{2}\left(\mathbb{R}^{n}, d x d t\right)} \sim\|\vec{f}\|_{L^{2}(\partial \Omega)}$ and $K(\nu, x, y, t)=\nabla \Gamma\left(\nu+P_{\gamma \nu} A(x, t)-A(y, t), x-y\right)$. We define

$$
T_{\nu} \vec{g}(x, t):=\int_{\mathbb{R}^{n-1}} K(\nu, x, y, t) \vec{g}(y, t) d y .
$$

Then $p \circ \rho(\nu, x, t)=T_{\nu} \vec{g}(x, t)$ and

$$
\left\|T_{\nu} \vec{g}\right\|_{L^{2}\left(\mathbb{R}^{n}\right)} \leq C\|\vec{f}\|_{L^{2}(\partial \Omega)}
$$

with a $C$ independent of $\nu$. We intend to prove that,

$$
\int_{0}^{\infty} \int_{\mathbb{R}^{n}}\left|Q_{\nu}^{(2)} T_{\nu} \vec{g}(x, t)\right|^{2} d x d t \frac{d \nu}{\nu} \leq C\|\vec{g}\|_{L^{2}\left(\mathbb{R}^{n}\right)}^{2} .
$$

Note that $Q_{\nu}^{(2)} T_{\nu}=T_{\nu} Q_{\nu}^{(2)}-\left[T_{\nu}, Q_{\nu}^{(2)}\right]$ and trivially

$$
\int_{0}^{\infty} \int_{\mathbb{R}^{n}}\left|T_{\nu} Q_{\nu}^{(2)} \vec{g}(x, t)\right|^{2} d x d t \frac{d \nu}{\nu} \leq C \int_{0}^{\infty} \int_{\mathbb{R}^{n}}\left|Q_{\nu}^{(2)} \vec{g}(x, t)\right|^{2} d x d t \frac{d \nu}{\nu} \leq C\|\vec{g}\|_{L^{2}\left(\mathbb{R}^{n}\right)}^{2} .
$$

We are therefore left with the commutator. Let $\psi_{\nu}^{(2)}$ be the kernel of $Q_{\nu}^{(2)}$.

$$
\left[T_{\nu}, Q_{\nu}^{(2)}\right] \vec{g}(x, t)=\int_{\mathbb{R}} \psi_{\nu}^{(2)}(t-s) \int_{\mathbb{R}^{n-1}}(K(\nu, x, y, t)-K(\nu, x, y, s)) \vec{g}(y, s) d y d s .
$$

Let $\tilde{K}(\nu, x, y, t, s)=K(\nu, x, y, t)-K(\nu, x, y, s)$ and decompose $\vec{g}(y, s)$, for fixed $\nu$, in the following way,

$$
\vec{g}(y, s)=P_{\nu} \vec{g}(y, s)+\left(I-P_{\nu}\right) \vec{g}(y, s)=P_{\nu} \vec{g}(y, s)+\int_{0}^{\nu} Q_{\mu}^{2} \vec{g}(y, s) \frac{d \mu}{\mu},
$$

where $P_{\nu}$ is a nice parabolic approximation to the identity. I.e., we have to consider

$$
\begin{aligned}
& M_{1}=\int_{\mathbb{R}} \int_{\mathbb{R}^{n-1}} \psi_{\nu}^{(2)}(t-s) \tilde{K}(\nu, x, y, t, s) P_{\nu} \vec{g}(y, s) d y d s, \\
& M_{2}=\int_{0}^{\nu} \int_{\mathbb{R}} \int_{\mathbb{R}^{n-1}} \psi_{\nu}^{(2)}(t-s) \tilde{K}(\nu, x, y, t, s) Q_{\mu}^{2} \vec{g}(y, s) d y d s \frac{d \mu}{\mu} .
\end{aligned}
$$

Define,

$$
A_{\nu, \mu}(x, t):=\int_{\mathbb{R}^{n-1}} \int_{\mathbb{R}^{n-1}} \psi_{\nu}^{(2)}(t-s) \tilde{K}(\nu, x, y, t, s) Q_{\mu}^{2} \vec{g}(y, s) d y d s .
$$

We now use the following lemma. The proof is postponed for now.

LEMMA 6.4 .

$$
\left\|A_{\nu, \mu}\right\|_{L^{2}\left(\mathbb{R}^{n}, d x d t\right)} \leq C\left(\frac{\mu}{\nu}\right)\left\|Q_{\mu} \vec{g}\right\|_{L^{2}\left(\mathbb{R}^{n}\right)}
$$


for some $C>0$ independent of $\vec{g}, \mu, \nu, \mu \leq \nu$.

Using the orthogonality statement in Lemma 6.4 and Littlewood-Paley theory we have,

$$
\begin{aligned}
\int_{0}^{\infty} \int_{\mathbb{R}^{n}}\left|\left[T_{\nu}, Q_{\nu}^{(2)}\right] \vec{g}(x, t)\right|^{2} d x d t \frac{d \nu}{\nu} & \leq \int_{0}^{\infty} \int_{\mathbb{R}^{n}}\left|M_{1}\right|^{2} d x d t \frac{d \nu}{\nu}+\int_{0}^{\infty} \int_{\mathbb{R}^{n}}\left|M_{2}\right|^{2} d x d t \frac{d \nu}{\nu} \\
& \lesssim \int_{0}^{\infty} \int_{\mathbb{R}^{n}}\left|M_{1}\right|^{2} d x d t \frac{d \nu}{\nu}+\|\vec{g}\|_{L^{2}\left(\mathbb{R}^{n}\right)}^{2}
\end{aligned}
$$

We now focus on $M_{1}$. Define

$$
\begin{aligned}
& H(\nu, x, y, t)=\nabla \Gamma\left(\nu+P_{\gamma \nu} A(x, t)-P_{\gamma \nu} A(y, t), x-y\right) \\
& \tilde{H}(\nu, x, y, t, s)=H(\nu, x, y, t)-H(\nu, x, y, s) .
\end{aligned}
$$

Then $M_{1}=M_{11}+M_{12}$ where,

$$
\begin{aligned}
M_{11} & =\int_{\mathbb{R}} \int_{\mathbb{R}^{n-1}} \psi_{\nu}^{(2)}(t-s) \tilde{H}(\nu, x, y, t, s) P_{\nu} \vec{g}(y, s) d y d s, \\
M_{12} & =\int_{\mathbb{R}} \int_{\mathbb{R}^{n-1}} \psi_{\nu}^{(2)}(t-s)(\tilde{K}(\nu, x, y, t, s)-\tilde{H}(\nu, x, y, t, s)) P_{\nu} \vec{g}(y, s) d y d s .
\end{aligned}
$$

Using calculus we may express $M_{11}$ in the following way,

$$
M_{11}=\int_{\mathbb{R}} \int_{\mathbb{R}^{n-1}} \psi_{\nu}^{(2)}(t-s)(t-s)\left[\int_{0}^{1} \frac{\partial}{\partial z_{n}} H(\nu, x, y, s+\theta(t-s)) d \theta\right] P_{\nu} \vec{g}(y, s) d y d s .
$$

Obviously

$$
\frac{\partial}{\partial z_{n}} H(\nu, x, y, t)=\nabla \Gamma_{x_{0}}\left(\nu+P_{\gamma \nu} A(x, t)-P_{\gamma \nu} A(y, t), x-y\right) \cdot \frac{\partial}{\partial t}\left(P_{\gamma \nu} A(x, t)-P_{\gamma \nu} A(y, t)\right) .
$$

As $\left|\partial^{2} \Gamma / \partial x_{i} \partial x_{j}\right| \leq c|x|^{-n}$ we may conclude that

$$
\begin{gathered}
\left|M_{11}\right| \leq C \int_{\mathbb{R}} \int_{\mathbb{R}^{n-1}} \frac{\left|\psi_{\nu}^{(2)}(t-s)\right||t-s|}{(\nu+|x-y|)^{n}} \\
\times\left[\int_{0}^{1}\left|\frac{\partial}{\partial z_{n}} P_{\gamma \nu} A(x, s+\theta(t-s))\right|+\left|\frac{\partial}{\partial z_{n}} P_{\gamma \nu} A(y, s+\theta(t-s))\right| d \theta\right] P_{\nu}|\vec{g}(y, s)| d y d s .
\end{gathered}
$$

Here $\frac{\partial}{\partial z_{n}}$ denotes the derivative in the $t$-direction. Assume without loss of generality $t-s>0,|t-s|<r^{2}$. Then

$$
\begin{aligned}
& \left|\int_{0}^{1}\right| \frac{\partial}{\partial z_{n}} P_{\gamma \nu} A(\cdot, s \pm \theta(t-s))|d \theta| \\
& \leq \frac{1}{|t-s|} \int_{0}^{|t-s|}\left|\frac{\partial}{\partial z_{n}} P_{\gamma \nu} A(\cdot, s \pm \theta)\right| d \theta \leq M_{n}^{\nu^{2}}\left(\frac{\partial}{\partial z_{n}} P_{\gamma \nu} A(\cdot,-)\right)(s) .
\end{aligned}
$$


I.e.,

$$
\begin{aligned}
\left|M_{11}\right| & \leq c M_{n}^{\nu^{2}}\left[\int_{\mathbb{R}^{n-1}} \frac{\nu^{2}}{(\nu+|x-y|)^{n}} M_{n}^{\nu^{2}}\left(\frac{\partial}{\partial z_{n}} P_{\gamma \nu} A(x,-)\right)(*) P_{\nu}|\vec{g}(y, *)| d y\right](t) \\
& +c M_{n}^{\nu^{2}}\left[\int_{\mathbb{R}^{n-1}} \frac{\nu^{2}}{(\nu+|x-y|)^{n}} M_{n}^{\nu^{2}}\left(\frac{\partial}{\partial z_{n}} P_{\gamma \nu} A(y,-)\right)(*) P_{\nu}|\vec{g}(y, *)| d y\right](t) \\
& \leq c M_{n}^{\nu^{2}}\left[\nu M^{(1)}\left(P_{\nu}|\vec{g}(\cdot, *)|\right)(x) M_{n}^{\nu^{2}}\left(\frac{\partial}{\partial z_{n}} P_{\gamma \nu} A(x,-)\right)(*)\right](t) \\
& +c M_{n}^{\nu^{2}}\left[\nu M^{(1)}\left[M_{n}^{\nu^{2}}\left(\frac{\partial}{\partial z_{n}} P_{\gamma \nu} A(\cdot,-)\right)(*) P_{\nu}|\vec{g}(\cdot, *)|\right](x)\right](t) .
\end{aligned}
$$

Using this estimate of $M_{11}$ and continuity of maximal functions we have,

$$
\begin{aligned}
& \int_{0}^{\infty} \int_{\mathbb{R}^{n}}\left|M_{11}\right|^{2} d x d t \frac{d \nu}{\nu} \\
\leq & C \int_{0}^{\infty} \int_{\mathbb{R}^{n}}\left|\nu M^{(1)}\left(P_{\nu}|\vec{g}(\cdot, t)|\right)(x) M_{n}^{\nu^{2}}\left(\frac{\partial}{\partial z_{n}} P_{\gamma \nu} A(x,-)\right)(t)\right|^{2} d x d t \frac{d \nu}{\nu} \\
+ & C \int_{0}^{\infty} \int_{\mathbb{R}^{n}}\left|\nu M^{(1)}\left[M_{n}^{\nu^{2}}\left(\frac{\partial}{\partial z_{n}} P_{\gamma \nu} A(\cdot,-)\right)(t) P_{\nu}|\vec{g}(\cdot, t)|\right](x)\right|^{2} d x d t \frac{d \nu}{\nu} .
\end{aligned}
$$

Using this estimate, the fact that according to Lemma 4.2 part (iii) the measure

$$
\left|\nu M_{n}^{\nu^{2}}\left(\frac{\partial P_{\gamma \nu} A(x,-)}{\partial z_{n}}\right)(t)\right|^{2} d x d t \frac{d \nu}{\nu}
$$

is a Carleson measure with norm no larger than $c \gamma^{-2}\left\|\mathbb{D}_{n} A\right\|_{*}^{2}$, the fact that $M^{(1)}\left(P_{\nu}(\vec{g}(\cdot, t))\right)(x) \leq P_{\nu}\left(M^{(1)}(\vec{g})\right)(x, t)$ we get as $P_{\nu} \vec{g}$ has good continuity with respect to $N_{*}$,

$$
\int_{0}^{\infty} \int_{\mathbb{R}^{n}}\left|M_{11}\right|^{2} d x d t \frac{d \nu}{\nu} \leq C\|\vec{g}\|_{2}^{2} .
$$

We now focus on $M_{12}$. Recall that

$$
M_{12}=\int_{\mathbb{R}} \int_{\mathbb{R}^{n-1}} \psi_{\nu}^{(2)}(t-s) \Theta(\nu, x, y, t, s) P_{\nu} \vec{g}(y, s) d y d s,
$$

where

$$
\begin{aligned}
& \Theta(\nu, x, y, t, s) \\
= & \nabla \Gamma\left(\nu+P_{\gamma \nu} A(x, t)-A(y, t), x-y\right)-\nabla \Gamma\left(\nu+P_{\gamma \nu} A(x, t)-P_{\gamma \nu} A(y, t), x-y\right) \\
+ & \nabla \Gamma\left(\nu+P_{\gamma \nu} A(x, s)-P_{\gamma \nu} A(y, s), x-y\right)-\nabla \Gamma\left(\nu+P_{\gamma \nu} A(x, s)-A(y, s), x-y\right) .
\end{aligned}
$$

I.e., by the mean-value theorem,

$$
|\Theta(\nu, x, y, t, s)| \leq \frac{\left|\left(I-P_{\gamma \nu}\right) A(y, t)\right|}{(\nu+|x-y|)^{n}}+\frac{\left|\left(I-P_{\gamma \nu}\right) A(y, s)\right|}{(\nu+|x-y|)^{n}} .
$$


Therefore,

$$
\begin{aligned}
\left|M_{12}\right| & \leq \int_{\mathbb{R}} \int_{\mathbb{R}^{n-1}} \frac{\left|\psi_{\nu}^{(2)}(t-s)\right|}{(\nu+|x-y|)^{n}}\left[\left|\left(I-P_{\gamma \nu}\right) A(y, t)\right|+\left|\left(I-P_{\gamma \nu}\right) A(y, s)\right|\right] P_{\nu}|\vec{g}(y, s)| d y d s \\
& \leq c M^{(1)}\left[\frac{1}{\nu}\left|\left(I-P_{\gamma \nu}\right) A(\cdot, t)\right|\left[\int_{\mathbb{R}}\left|\psi_{\nu}^{(2)}(t-s)\right| P_{\nu}|\vec{g}(\cdot, s)| d s\right]\right](x) \\
& +c M_{n}\left[M^{(1)}\left[\frac{1}{\nu} \mid\left(I-P_{\gamma \nu}\right) A(\cdot, *) P_{\nu} \vec{g}(\cdot, *)\right](x)\right](t):=M_{121}+M_{122} .
\end{aligned}
$$

But

$$
\left(\frac{1}{\nu}\left|\left(I-P_{\gamma \nu}\right) A(x, t)\right|\right)^{2} d x d t \frac{d \nu}{\nu}
$$

is a Carleson measure with norm no greater that $c \gamma^{2}\|A\|_{\text {comm }}$. This is statement (iv) of Lemma 4.2. Therefore by continuity of maximal functions we have the desired estimate for $M_{122}$. Left to estimate is

$$
\int_{\mathbb{R}} \int_{\mathbb{R}^{n}} M_{121}^{2} d x d t \frac{d \nu}{\nu}
$$

But

$$
\begin{aligned}
\int_{\mathbb{R}^{n}} M_{121}^{2} d x d t & \leq C \int_{\mathbb{R}^{n}}\left|\frac{1}{\nu}\right|\left(I-P_{\gamma \nu}\right) A(x, t)\left|\int_{\mathbb{R}}\right| \psi_{\nu}^{(2)}(t-s)\left|P_{\nu}\right| \vec{g}(x, s)|d s|^{2} d x d t \\
& \leq\left. C \int_{\mathbb{R}^{n}}\left|\psi_{\nu}^{(2)}\right| *\left|\frac{1}{\nu}\right|\left(I-P_{\gamma \nu}\right) A\right|^{2}\left|P_{\nu}\right| \vec{g}||^{2} d x d t .
\end{aligned}
$$

Again, as is easily seen, the measure

$$
\left.\left|\psi_{\nu}^{(2)}\right| *\left|\frac{1}{\nu}\right|\left(I-P_{\gamma \nu}\right) A\right|^{2}(x, t) d x d t \frac{d \nu}{\nu}
$$

is a Carleson measure with norm $\leq c \gamma^{2}\|A\|_{\text {comm }}$. Using this and the fact that the non-tangential maximal function of $P_{\nu}|\vec{g}|$ is good we may complete the proof. I.e., the proof of Lemma 6.2 is complete modulo Lemma 6.4.

Proof of Lemma 6.4. Set

$$
\begin{aligned}
& V_{\nu}(x, y, t, s)=\psi_{\nu}^{(2)}(t-s) \tilde{K}(\nu, x, y, t, s) \\
& =\psi_{\nu}^{(2)}(t-s)\left[\nabla \Gamma\left(\nu+P_{\gamma \nu} A(x, t)-A(y, t), x-y\right)-\nabla \Gamma\left(\nu+P_{\gamma \nu} A(x, s)-A(y, s), x-y\right)\right] .
\end{aligned}
$$

Using that if $\|(y, s)-(\tilde{y}, \tilde{s})\| \leq \mu$ then,

$$
V_{\nu}(x, y, t, s)-V_{\nu}(x, \tilde{y}, t, \tilde{s}) \leq c_{\beta} \frac{\mu}{\nu}\left[\frac{1}{\nu^{2}} \chi_{\left\{|t-s|<\nu^{2}\right\}}(t) \cdot \frac{\nu}{(\nu+|x-y|)^{n}}\right],
$$

we may conclude that

$$
\left|A_{\nu, \mu}(x, t)\right|:=\left|\int_{\mathbb{R}} \int_{\mathbb{R}^{n-1}} \psi_{\nu}^{(2)}(t-s) \tilde{K}(\nu, x, y, t, s) Q_{\mu}^{2} \vec{g}(y, s) d y d s\right|
$$




$$
\begin{aligned}
& =\left|\int_{\mathbb{R}} \int_{\mathbb{R}^{n-1}} Q_{\mu} V_{\nu}(x, y, t, s) Q_{\mu} \vec{g}(y, s) d y d s\right| \\
& \leq C_{\beta} \frac{\mu}{\nu} M_{n}\left(M^{(1)}\left(Q_{\mu} \vec{g}(\cdot, *)(x)\right)\right)(t) .
\end{aligned}
$$

Continuity of maximal functions now completes the proof.

Proof of Lemma 6.3. Again the kernel of $H D_{1 / 2}^{t}$ is $k(t)=\operatorname{csgn}(t)|t|^{-3 / 2}$. Let

$$
k_{1}(t)=k(t) \chi_{\left\{s:|s| \leq R^{2} \nu \lambda\right\}}(t), \quad k_{2}(t)=k(t) \chi_{\left\{s:|s|>R^{2} \nu \lambda\right\}}(t),
$$

where $R, \nu, \lambda>0$ and let $D_{1}$ and $D_{2}$ be the associated operators. Using part (i) of Lemma 4.1 we have with $R$ replaced with $\tilde{R}$ such that $\tilde{R}^{2} \lambda^{2}=R^{2} \nu \lambda$,

$$
\begin{aligned}
\left|\lambda\left[D_{1}, \frac{\partial P_{\gamma \lambda} A}{\partial x_{r}}\right] g(x, t)\right| & \leq c_{\beta} \tilde{R} \gamma^{-2}\left\|\mathbb{D}_{n} A\right\|_{*} M_{n}(g(x, \cdot))(t) \\
& \leq c_{\beta} R \gamma^{-2}\left\|\mathbb{D}_{n} A\right\|_{*}\left(\frac{\nu}{\lambda}\right)^{1 / 2} M_{n}(g(x, \cdot))(t) .
\end{aligned}
$$

Again $M_{n}$ is the Hardy-Littlewood maximal function in the t-variable. As

$$
\left|\left[Q_{\nu}^{(2)}\left[D_{1}, \frac{\partial P_{\gamma \lambda} A}{\partial x_{r}}\right]\left(u^{r} \circ \rho\right)_{\lambda \lambda}\right](\lambda, x, t)\right| \leq C M_{n}\left[\left(\left[D_{1}, \frac{\partial P_{\gamma \lambda} A}{\partial x_{r}}\right]\left(u^{r} \circ \rho\right)_{\lambda \lambda}\right)(\lambda, x, \cdot)\right](t),
$$

we have,

$$
\begin{aligned}
\int_{0}^{\infty} \int_{\mathbb{R}^{n}} \int_{0}^{\lambda}\left|Q_{\nu}^{(2)}\left[D_{1}, \frac{\partial P_{\gamma \lambda} A}{\partial x_{r}}\right]\left(u^{r} \circ \rho\right)_{\lambda \lambda}\right|^{2}\left(\frac{\nu}{\lambda}\right)^{-\epsilon} d z \frac{d \nu}{\nu} \lambda^{3} d \lambda \\
\lesssim R^{2} \gamma^{-4}\left\|\mathbb{D}_{n} A\right\|_{*}^{2} \int_{0}^{\infty} \int_{\mathbb{R}^{n}} \int_{0}^{\lambda}\left|M_{n}\left(\left(u^{r} \circ \rho\right)_{\lambda \lambda}(x, \cdot)\right)(t)\right|^{2}\left(\frac{\nu}{\lambda}\right)^{1-\epsilon} d z \frac{d \nu}{\nu} \lambda d \lambda \lesssim \gamma\|\vec{f}\|_{2}^{2},
\end{aligned}
$$

with an appropriate choice of $R$ and an application of Lemma 1.1, Lemma 2.4 and Theorem 3.1. Next we consider the part of $B_{22}$ coming from the operator $D_{2}$.

$$
\begin{aligned}
Q_{\nu}^{(2)}\left[D_{2}, \frac{\partial P_{\gamma \lambda} A}{\partial x_{r}}\right] & =Q_{\nu}^{(2)} D_{2} \frac{\partial P_{\gamma \lambda} A}{\partial x_{r}}+\frac{\partial P_{\gamma \lambda} A}{\partial x_{r}} Q_{\nu}^{(2)} D_{2}+\left[Q_{\nu}^{(2)}, \frac{\partial P_{\gamma \lambda} A}{\partial x_{r}}\right] D_{2} \\
& =S_{1}+S_{2}+S_{3} .
\end{aligned}
$$

Let us consider an expression of the form $\lambda Q_{\nu}^{(2)} D_{2} g$. Standard deductions give,

$$
\left|\lambda Q_{\nu}^{(2)} D_{2} g(x, t)\right| \leq C R^{-3}\left(\frac{\nu}{\lambda}\right)^{1 / 2} M_{n}(g(x, \cdot)(t)
$$

I.e., the contribution from the operators $S_{1}$ and $S_{2}$ to expression under consideration can be handled. We now focus on the commutator part and we let in the following $\psi_{\nu}^{(2)}$ be the kernel of $Q_{\nu}^{(2)}$.

$$
\begin{aligned}
& \left|\left[Q_{\nu}^{(2)}, \frac{\partial P_{\gamma \lambda} A}{\partial x_{r}}\right] g\right| \\
= & \left|\int_{\mathbb{R}} \psi_{\nu}^{(2)}(t-s)\left(\frac{\partial P_{\gamma \lambda} A(x, s)}{\partial x_{r}}-\frac{\partial P_{\gamma \lambda} A(x, t)}{\partial x_{r}}\right) g(x, s) d s\right|
\end{aligned}
$$




$$
\begin{aligned}
& =\left|\int_{0}^{1} \int_{\left\{s:|s-t| \leq \nu^{2}\right\}} \psi_{\nu}^{(2)}(t-s)(t-s) \frac{\partial^{2} P_{\gamma \lambda} A(x, s+\theta(t-s))}{\partial z_{n} \partial x_{r}} g(x, s) d s d \theta\right| \\
& \leq c \nu^{2} \gamma^{-2}\left\|\mathbb{D}_{n} A\right\|_{*} \lambda^{-2} \int_{\left\{s:|s-t| \leq \nu^{2}\right\}}\left|\psi_{\nu}^{(2)}(t-s)\right||g(x, s)| d s \\
& \leq c\left(\frac{\nu}{\lambda}\right)^{1 / 2} \gamma^{2}\left\|\mathbb{D}_{n} A\right\|_{*} M_{n}(g(x, \cdot))(t) .
\end{aligned}
$$

Furthermore

$$
\left|\lambda D_{2} g\right| \leq R^{-1}\left(\frac{\lambda}{\nu}\right)^{1 / 2} \tilde{\phi}_{R \nu^{1 / 2} \lambda^{1 / 2}} * g \leq c R^{-1}\left(\frac{\lambda}{\nu}\right)^{1 / 2} M_{n}(g(x, \cdot)),
$$

where

$$
\phi_{R \nu^{1 / 2} \lambda^{1 / 2}}(t)=\frac{R \nu^{1 / 2} \lambda^{1 / 2}}{|t|^{3 / 2}} \chi_{\left\{|t|>R^{2} \nu \lambda\right\}}(t) .
$$

Combining these estimates and appealing to the continuity of $M_{n}$ we have completed the proof of the estimate stated in the lemma.

7. Estimates for the pressure. The main identity explored in this section and stated in Lemma 7.4 below was, as far as we know, first discovered and used in this context by Shen [32]. Let in the following,

$$
\begin{aligned}
& \Omega^{\epsilon}=\left\{\left(x_{0}, x, t\right): x_{0}>\epsilon+P_{\gamma \epsilon} A(x, t)\right\}, \\
& \Omega_{t_{0}}^{\epsilon}=\Omega^{\epsilon} \cap\left\{t=t_{0}\right\}=\left\{\left(x_{0}, x\right): x_{0}>\epsilon+P_{\gamma \epsilon} A\left(x, t_{0}\right)\right\} .
\end{aligned}
$$

Note that if $\epsilon_{2}<\epsilon_{1}$ then $\epsilon_{1}+P_{\gamma \epsilon_{1}} A\left(x, t_{0}\right)>\epsilon_{2}+P_{\gamma \epsilon_{2}} A\left(x, t_{0}\right)$. I.e., $\Omega_{t_{0}}^{\epsilon_{2}} \subset \Omega_{t_{0}}^{\epsilon_{1}}$ and $\Omega_{t_{0}}^{\epsilon} \nearrow \Omega_{t_{0}}$ for each $t_{0}$ as $\epsilon \rightarrow 0$. Obviously we also have that, $\Omega^{\epsilon} \nearrow \Omega$ as $\epsilon \rightarrow 0$. $N_{t}^{\epsilon}$ is the unit outer normal to $\partial \Omega_{t}^{\epsilon}$. $N_{t}^{\epsilon, j}$ is the $j$-te component of $N_{t}^{\epsilon}$. Let $S_{t}^{\epsilon} f$ be the singular layer potential associated to the Laplace operator in the domain $\Omega_{t}^{\epsilon}$. The first result is the following and is essentially due to Shen[30],

Lemma 7.1. Let $\vec{v} \in C^{2}\left(\mathbb{R}^{n+1} \backslash \partial \Omega\right)$ and div $\vec{v}=0$ in $\mathbb{R}^{n+1} \backslash \partial \Omega$. Then,

$$
\| S_{t}^{\epsilon}\left(\left\langle\left(\Delta \vec{v}, N_{t}^{\epsilon}\right\rangle\right)\left\|_{L^{2}\left(\partial \Omega_{t}^{\epsilon}\right)} \leq C\right\| \nabla \vec{v} \|_{L^{2}\left(\partial \Omega_{t}^{\epsilon}\right)} .\right.
$$

Here $C$ is independent of $t$ and $\epsilon$.

In order to formulate the other two results we need to introduce some more notation. Define for $\delta>0$,

$$
\begin{aligned}
\Omega^{\epsilon, \delta} & =\left\{\left(x_{0}, x, t\right): x_{0}>\delta+\epsilon+P_{\gamma \epsilon} A(x, t)\right\}, \\
\Omega^{\epsilon,-\delta} & =\left\{\left(x_{0}, x, t\right): x_{0}<-\delta+\epsilon+P_{\gamma \epsilon} A(x, t)\right\} .
\end{aligned}
$$

In the following we let $S_{t}^{\epsilon, \pm \delta}$ denote the singular layer potential associated to the Laplace operator on $\Omega_{t}^{\epsilon, \pm \delta}$. $N_{t}^{\epsilon, \pm \delta}$ is the unit outer normal to $\partial \Omega_{t}^{\epsilon \pm \delta}$. We also introduce the maps,

$$
\rho_{\epsilon, \delta}(\epsilon, x, t)=\left(\delta+\epsilon+P_{\gamma \epsilon} A(x, t), x, t\right) \quad \rho_{\epsilon,-\delta}(\epsilon, x, t)=\left(-\delta+\epsilon+P_{\gamma \epsilon} A(x, t), x, t\right) .
$$

We will prove the following two lemmas, 
Lemma 7.2. Let in the following $(\vec{u}, p)=\left(S^{\epsilon} \vec{f}, p^{\epsilon}\right)=(S \vec{f}, p)$ be the singular layer potential and pressure defined with $\Omega^{\epsilon}$ as the domain of reference and let $\vec{f} \in$ $C_{0}^{\infty}\left(\mathbb{R}^{n+1}\right)$. Then the following inequalities are valid with a constant $C$ independent of $\epsilon$ and $\vec{f}$,

(i) $\quad\left\|p_{+}\right\|_{L^{2}\left(\partial \Omega^{\epsilon}\right)}$

$$
\leq C\left[\left\|\nabla \vec{u}_{+}\right\|_{L^{2}\left(\partial \Omega^{\epsilon}\right)}+\left(\lim _{\delta \rightarrow 0^{+}} \int_{-\infty}^{\infty} \int_{\partial \Omega_{t}^{\epsilon, \delta}}\left|S_{t}^{\epsilon, \delta}\left(\left\langle\frac{\partial \vec{u}}{\partial t}, N_{t}^{\epsilon, \delta}\right\rangle\right)\right|^{2} d \sigma_{t}^{\epsilon, \delta} d t\right)^{1 / 2}\right],
$$

(ii) $\quad\left\|p_{-}\right\|_{L^{2}\left(\partial \Omega^{\epsilon}\right)}$

$$
\leq C\left[\left\|\nabla \vec{u}_{-}\right\|_{L^{2}\left(\partial \Omega^{\epsilon}\right)}+\left(\lim _{\delta \rightarrow 0^{+}} \int_{-\infty}^{\infty} \int_{\partial \Omega_{t}^{\epsilon,-\delta}}\left|S_{t}^{\epsilon,-\delta}\left(\left\langle\frac{\partial \vec{u}}{\partial t}, N_{t}^{\epsilon, \delta}\right\rangle\right)\right|^{2} d \sigma_{t}^{\epsilon,-\delta} d t\right)^{1 / 2}\right],
$$

(iii)

$$
\begin{aligned}
& \left(\lim _{\delta \rightarrow 0^{+}} \int_{-\infty}^{\infty} \int_{\Omega_{t}^{\epsilon, \pm \delta}}\left|S_{t}^{\epsilon, \pm \delta}\left(\left\langle\frac{\partial \vec{u}}{\partial t}, N_{t}^{\epsilon,-\delta}\right\rangle\right)\right|^{2} d \sigma_{t}^{\epsilon,-\delta} d t\right)^{1 / 2} \\
\leq & C\left[\left\|p_{ \pm}\right\|_{L^{2}\left(\partial \Omega^{\epsilon}\right)}+\left\|\nabla \vec{u}_{ \pm}\right\|_{L^{2}\left(\partial \Omega^{\epsilon}\right)}\right] .
\end{aligned}
$$

Lemma 7.3. Let in the following $(\vec{u}, p)=\left(S^{\epsilon} \vec{f}, p^{\epsilon}\right)=(S \vec{f}, p)$ be the singular layer potential and pressure defined with $\Omega^{\epsilon}$ as the domain of reference and let $\vec{f} \in$ $C_{0}^{\infty}\left(\mathbb{R}^{n+1}\right)$. Then,

$\lim _{\delta \rightarrow 0^{+}} \int_{-\infty}^{\infty} \int_{\partial \Omega_{t}^{\epsilon, \delta}}\left|S_{t}^{\epsilon, \delta}\left(\left\langle\frac{\partial \vec{u}}{\partial t}, N_{t}^{\epsilon, \delta}\right\rangle\right)\right|^{2} d \sigma_{t}^{\epsilon, \delta} d t=\lim _{\delta \rightarrow 0^{+}} \int_{-\infty}^{\infty} \int_{\partial \Omega_{t}^{\epsilon,-\delta}}\left|S_{t}^{\epsilon,-\delta}\left(\left\langle\frac{\partial \vec{u}}{\partial t}, N_{t}^{\epsilon, \delta}\right\rangle\right)\right|^{2} d \sigma_{t}^{\epsilon,-\delta} d t$ and

$$
\lim _{\delta \rightarrow 0^{+}} \int_{-\infty}^{\infty} \int_{\partial \Omega_{t}^{\epsilon, \delta}}\left|S_{t}^{\epsilon, \delta}\left(\left\langle\frac{\partial \vec{u}}{\partial t}, N_{t}^{\epsilon, \delta}\right\rangle\right)\right|^{2} d \sigma_{t}^{\epsilon, \delta} d t=\int_{-\infty}^{\infty} \int_{\partial \Omega_{t}^{\epsilon}}\left|S_{t}^{\epsilon}\left(\left\langle\frac{\partial \vec{u}}{\partial t}, N_{t}^{\epsilon}\right\rangle\right)\right|^{2} d \sigma_{t}^{\epsilon} d t
$$

Let in the following $(\vec{u}, p)=\left(S^{\epsilon} \vec{f}, p^{\epsilon}\right)=(S \vec{f}, p)$ be the singular layer potential and pressure defined with $\Omega^{\epsilon}$ as the domain of reference and let $\vec{f} \in L^{2}\left(\partial \Omega^{\epsilon}\right)$. Let $\overrightarrow{f_{j}} \in C_{0}^{\infty}\left(\mathbb{R}^{n+1}\right)$ be such that $\overrightarrow{f_{j}} \rightarrow \vec{f}$ in $L^{2}\left(\partial \Omega^{\epsilon}\right)$. Using Lemma 7.2 and 7.3 we define,

$$
S_{t}^{\epsilon}\left(\left\langle\frac{\partial S \vec{f}}{\partial t}, N_{t}^{\epsilon}\right\rangle\right)=\lim _{j \rightarrow \infty} S_{t}^{\epsilon}\left(\left\langle\frac{\partial S \vec{f}_{j}}{\partial t}, N_{t}^{\epsilon}\right\rangle\right)
$$

with convergence in $L^{2}\left(\partial \Omega^{\epsilon}\right)$. Obviously the definition is independent of the sequence $f_{j}$. The quantity $S_{t}^{\epsilon}\left(\left\langle\frac{\partial S \vec{f}}{\partial t}, N_{t}^{\epsilon}\right\rangle\right)$ is therefore well defined for every $\vec{f} \in L^{2}\left(\partial \Omega^{\epsilon}\right)$.

We end the section with the proof of Lemma 7.1-7.3.

Proof of Lemma 7.1. $\left\langle\Delta \vec{v}, N_{t}^{\epsilon}\right\rangle=\sum_{i=0}^{n-1} \Delta v^{i} N_{t}^{\epsilon, i}$. Here,

$$
N_{t}^{\epsilon, i}=\frac{\partial P_{\gamma \epsilon} A}{\partial x_{i}} \cdot \frac{1}{\left|N_{t}^{\epsilon}\right|} \text { for } i \in\{1, \ldots, n-1\}, \quad N_{t}^{\epsilon, 0}=-\frac{1}{\left|N_{t}^{\epsilon}\right|} .
$$


Note that on $\partial \Omega_{t}^{\epsilon}$ we have $x_{0}=\epsilon+P_{\gamma \epsilon} A\left(x, t_{0}\right)$. Let

$$
T_{j}:=\left(\frac{\partial P_{\gamma \epsilon} A}{\partial x_{j}}, 0,,,,, 0,1,0, \ldots 0\right) /\left|N_{t}^{\epsilon}\right|,
$$

where we have placed the number 1 at position $j$. Here $j \in\{1, \ldots, n-1\}$. Note that $T_{j}$ is tangent to $\partial \Omega_{t}^{\epsilon}$. A direct calculation using that div $\vec{u}=0$ shows that the following formula is true.

LEMMA 7.4.

$$
\begin{aligned}
\left\langle\Delta \vec{v}, N_{t}^{\epsilon}\right\rangle\left|N_{t}^{\epsilon}\right| & =\left[\sum_{j, k=1}^{n-1} \frac{\partial}{\partial T_{j}}\left(\frac{\partial v^{k}}{\partial x_{j}} \frac{\partial P_{\gamma \epsilon} A}{\partial x_{k}}\right)-\frac{\partial}{\partial T_{k}}\left(\frac{\partial v^{k}}{\partial x_{j}} \frac{\partial P_{\gamma \epsilon} A}{\partial x_{j}}\right)\right. \\
& \left.-\sum_{j=1}^{n-1} \frac{\partial}{\partial T_{j}}\left(\frac{\partial v^{0}}{\partial x_{j}}\right)+\sum_{k=1}^{n-1} \frac{\partial}{\partial T_{k}}\left(\frac{\partial v^{k}}{\partial x_{0}}\right)\right]\left|N_{t}^{\epsilon}\right| .
\end{aligned}
$$

Using Lemma 7.4 we now consider the singular integrals we want to estimate, i.e.,

$$
\begin{aligned}
& S_{t}^{\epsilon}\left(\left\langle\Delta \vec{v}, N_{t}^{\epsilon}\right\rangle\right)(P, t) \\
= & p \cdot v \int_{\partial \Omega_{t}^{\epsilon}} \frac{\left\langle\Delta \vec{v}, N_{t}^{\epsilon}\right\rangle(Q, t)}{w_{n}(2-n)|P-Q|^{n-2}} d \sigma_{t}^{\epsilon}(Q) \\
= & \sum_{j, k=1}^{n-1} \int_{\partial \Omega_{t}^{\epsilon}} \frac{1}{w_{n}(2-n)|P-Q|^{n-2}}\left[\frac{\partial}{\partial T_{j}}\left(\frac{\partial v^{k}}{\partial x_{j}} \frac{\partial P_{\gamma \epsilon} A}{\partial x_{k}}\right)-\frac{\partial}{\partial T_{k}}\left(\frac{\partial v^{k}}{\partial x_{j}} \frac{\partial P_{\gamma \epsilon} A}{\partial x_{j}}\right)\right] d \sigma_{t}^{\epsilon} \\
- & \sum_{j=1}^{n-1} \int_{\partial \Omega_{t}^{\epsilon}} \frac{1}{w_{n}(2-n)|P-Q|^{n-2}} \frac{\partial}{\partial T_{j}}\left(\frac{\partial v^{0}}{\partial x_{j}}\right) d \sigma_{t}^{\epsilon} \\
+ & \left.\sum_{k=1}^{n-1} \int_{\partial \Omega_{t}^{\epsilon}} \frac{\partial}{w_{n}(2-n)|P-Q|^{n-2}} \frac{\partial v_{k}^{k}}{\partial T_{k}}\right) d \sigma_{t}^{\epsilon} .
\end{aligned}
$$

Using integration by parts along $\partial \Omega_{t}^{\epsilon}$ and regularity estimates for the Dirichlet problem on Lipschitz domains, see e.g. [30], we may conclude that,

$$
\| S_{t}^{\epsilon}\left(\left\langle\Delta \vec{v}, N_{t}^{\epsilon}\right\rangle\left\|_{L^{2}\left(\partial \Omega_{t}^{\epsilon}\right)} \leq C\right\| \nabla \vec{v} \|_{L^{2}\left(\partial \Omega_{t}^{\epsilon}\right)}\right.
$$

with $C$ as in the statement of the Lemma.

Proof of Lemma 7.2. We start out by proving (i). As $p$ is harmonic and $\partial \Omega_{t}^{\epsilon}$ is smooth we have using the Gauss Green formula that

$$
\begin{aligned}
p(X, t) & =\int_{\partial \Omega_{t}^{\epsilon, \delta}} \frac{\left\langle Q-X, N_{t}^{\epsilon, \delta}(Q)\right\rangle(Q, t)}{|X-Q|^{n}} p(Q, t) d \sigma_{t}^{\epsilon, \delta}(Q) \\
& -\int_{\partial \Omega_{t}^{\epsilon, \delta}} \frac{\frac{\partial p}{\partial N_{t}^{\epsilon, \delta}}(Q, t)}{w_{n}(2-n)|X-Q|^{n-2}} d \sigma_{t}^{\epsilon, \delta}(Q) .
\end{aligned}
$$


Here $d \sigma_{t}^{\epsilon, \delta}, N_{t}^{\epsilon, \delta}$ are naturally defined. Let $S_{t}^{\epsilon, \delta}$ be the singular layer potential operators for the Laplace operator on $\Omega_{t}^{\epsilon, \delta}$. If we let $X \rightarrow P \in \partial \Omega_{t}^{\epsilon, \delta}$ we have the relation

$$
p(P, t)=\frac{1}{2} p(P, t)+K_{t}^{\epsilon, \delta}(p)(P, t)+S_{t}^{\epsilon, \delta}\left(\frac{\partial p}{\partial N_{t}^{\epsilon, \delta}}\right)(P, t) .
$$

Here,

$$
K_{t}^{\epsilon, \delta}(p)(P, t):=p . v \int_{\partial \Omega_{t}^{\epsilon, \delta}} \frac{\left\langle P-Q, N_{t}^{\epsilon, \delta}(Q)\right\rangle(Q, t)}{w_{n}|P-Q|^{n}} p(Q, t) d \sigma_{t}^{\epsilon, \delta}(Q) .
$$

I.e., $S_{t}^{\epsilon, \delta}\left(\frac{\partial p}{\partial N_{t}^{\epsilon, \delta}}\right)(P, t)=\left(\frac{I}{2}-K_{t}^{\epsilon, \delta}\right)(p)(P, t)$. Therefore,

$$
\begin{gathered}
\left\|\left(\frac{I}{2}-K_{t}^{\epsilon, \delta}\right)(p)\right\|_{L^{2}\left(\partial \Omega_{t}^{\epsilon, \delta}\right)}=\| S_{t}^{\epsilon, \delta}\left(\frac{\partial p}{\left.\partial N_{t}^{\epsilon, \delta}\right)} \|_{L^{2}\left(\partial \Omega_{t}^{\epsilon, \delta}\right)}\right. \\
\leq C\left(\left\|S_{t}^{\epsilon, \delta}\left(\left\langle\Delta u, N_{t}^{\epsilon, \delta}\right\rangle\right)\right\|_{L^{2}\left(\partial \Omega_{t}^{\epsilon, \delta}\right)}+\left\|S_{t}^{\epsilon, \delta}\left(\left\langle\frac{\partial \vec{u}}{\partial t}, N_{t}^{\epsilon, \delta}\right\rangle\right)\right\|_{L^{2}\left(\partial \Omega_{t}^{\epsilon, \delta}\right)} .\right.
\end{gathered}
$$

As $\Omega_{t}^{\epsilon, \delta}$ is an unbounded Lipschitz domain it follows from the invertibility of $\frac{I}{2}-K_{t}^{\epsilon, \delta}$ that,

$$
\|p\|_{L^{2}\left(\partial \Omega_{t}^{\epsilon, \delta}\right)} \leq C\left\|\left(\frac{I}{2}-K_{t}^{\epsilon, \delta}\right)(p)\right\|_{L^{2}\left(\partial \Omega_{t}^{\epsilon, \delta}\right)} .
$$

I.e. by continuity and Lemma 7.1,

$$
\|p\|_{L^{2}\left(\partial \Omega^{\epsilon}\right)} \leq C\left[\|\nabla \vec{u}\|_{L^{2}\left(\partial \Omega^{\epsilon}\right)}+\left(\lim _{\delta \rightarrow 0} \int_{-\infty}^{\infty}\left\|S_{t}^{\epsilon, \delta}\left(\left\langle\frac{\partial \vec{u}}{\partial t}, N_{t}^{\epsilon, \delta}\right\rangle\right)\right\|_{L^{2}\left(\partial \Omega_{t}^{\epsilon, \delta}\right)}^{2} d t\right)^{1 / 2}\right] .
$$

All of the constants in these inequalities are independent of $\epsilon$ and $\delta$.

The proof of (ii) follows the same lines as the proof of part (i) with the modification that we instead work with domains,

$$
\Omega^{\epsilon,-\delta}=\left\{\left(x_{0}, x, t\right): x_{0}<-\delta+\epsilon+P_{\gamma \epsilon} A(x, t)\right\} .
$$

We now prove (iii). We choose to consider the case of $\Omega^{\epsilon,-\delta}$. Then

$$
\begin{aligned}
& \int_{-\infty}^{\infty}\left\|S_{t}^{\epsilon,-\delta}\left(\left\langle\frac{\partial \vec{u}}{\partial t}, N_{t}^{\epsilon,-\delta}\right\rangle\right)\right\|_{L^{2}\left(\partial \Omega_{t}^{\epsilon,-\delta}\right)}^{2} d t \\
\leq & \int_{-\infty}^{\infty} \int_{\Omega_{t}^{\epsilon,-\delta}}\left|S_{t}^{\epsilon,-\delta}\left(\left\langle\Delta \vec{u}, N_{t}^{\epsilon,-\delta}\right\rangle\right)\right|^{2} d \sigma_{t}^{\epsilon,-\delta} d t+\int_{-\infty}^{\infty} \int_{\Omega_{t}^{\epsilon,-\delta}}\left|S_{t}^{\epsilon,-\delta}\left(\frac{\partial p}{\partial N_{t}^{\epsilon,-\delta}}\right)\right|^{2} d \sigma_{t}^{\epsilon,-\delta} d t \\
\leq & C\left(\left\|\nabla \vec{u}_{-}\right\|_{L^{2}\left(\partial \Omega^{\epsilon,-\delta}\right)}+\left\|\left(\frac{I}{2}-K_{t}^{\epsilon,-\delta}\right)(p)\right\|_{L^{2}\left(\partial \Omega^{\epsilon,-\delta}\right)}\right. \\
\leq & C\left(\left\|\nabla \vec{u}_{-}\right\|_{L^{2}\left(\partial \Omega^{\epsilon,-\delta}\right)}+\left\|p_{-}\right\|_{L^{2}\left(\partial \Omega^{\epsilon,-\delta}\right)}\right) .
\end{aligned}
$$

Arguing as above we may complete the proof. 
Proof of Lemma 7.3.

$$
\begin{aligned}
& S^{\epsilon} \vec{f}(X, t):=\int_{-\infty}^{t} \int_{\partial \Omega_{s}^{\epsilon}} \Gamma(X-Y, t-s) \vec{f}(Y, s) d \sigma_{s}^{\epsilon}(Y) d s \\
& =\int_{-\infty}^{t} \int_{\mathbb{R}^{n-1}} \Gamma\left(x_{0}-\left(\epsilon+P_{\gamma \epsilon} A(y, s)\right), x-y, t-s\right) \vec{f}\left(\epsilon+P_{\gamma \epsilon} A(y, s), y, s\right) \\
& \quad \times \sqrt{1+\left|\nabla P_{\gamma \epsilon} A(y, s)\right|^{2}} d y d s .
\end{aligned}
$$

I.e.,

$$
\frac{\partial S^{\epsilon}(\vec{f})}{\partial t}(X, t)=\int_{-\infty}^{t} \int_{\mathbb{R}^{n-1}} \frac{\partial \Gamma}{\partial t}\left(x_{0}-\left(\epsilon+P_{\gamma \epsilon} A(y, s)\right), x-y, t-s\right) \vec{g}(y, s) d y d s,
$$

where,

$$
\vec{g}(y, s)=\vec{f}\left(\epsilon+P_{\gamma \epsilon} A(y, s), y, s\right) \sqrt{1+\left|\nabla P_{\gamma \epsilon} A(y, s)\right|^{2}} .
$$

But,

$$
\begin{aligned}
& \frac{\partial}{\partial s} \Gamma\left(x_{0}-\left(\epsilon+P_{\gamma \epsilon} A(y, s)\right), x-y, t-s\right) \\
= & -\frac{\partial \Gamma}{\partial x_{0}}\left(x_{0}-\left(\epsilon+P_{\gamma \epsilon} A(y, s)\right), x-y, t-s\right) \frac{\partial P_{\gamma \epsilon} A(y, s)}{\partial s} \\
& -\frac{\partial \Gamma}{\partial t}\left(x_{0}-\left(\epsilon+P_{\gamma \epsilon} A(y, s)\right), x-y, t-s\right) .
\end{aligned}
$$

Therefore,

$$
\begin{aligned}
& \frac{\partial S^{\epsilon}(\vec{f})}{\partial t}(X, t) \\
= & -\int_{-\infty}^{t} \int_{\mathbb{R}^{n-1}} \frac{\partial \Gamma}{\partial x_{0}}\left(x_{0}-\left(\epsilon+P_{\gamma \epsilon} A(y, s)\right), x-y, t-s\right) \frac{\partial P_{\gamma \epsilon} A(y, s)}{\partial s} \vec{g}(y, s) d y d s \\
& -\int_{-\infty}^{t} \int_{\mathbb{R}^{n-1}} \frac{\partial}{\partial s} \Gamma\left(x_{0}-\left(\epsilon+P_{\gamma \epsilon} A(y, s)\right), x-y, t-s\right) \vec{g}(y, s) d y d s \\
= & -\int_{-\infty}^{t} \int_{\mathbb{R}^{n-1}} \frac{\partial \Gamma}{\partial x_{0}}\left(x_{0}-\left(\epsilon+P_{\gamma \epsilon} A(y, s)\right), x-y, t-s\right) \frac{\partial P_{\gamma \epsilon} A(y, s)}{\partial s} \vec{g}(y, s) d y d s \\
& +\int_{-\infty}^{t} \int_{\mathbb{R}^{n-1}} \Gamma\left(x_{0}-\left(\epsilon+P_{\gamma \epsilon} A(y, s)\right), x-y, t-s\right) \frac{\partial \vec{g}(y, s)}{\partial s} d y d s .
\end{aligned}
$$

We introduce the following notation,

$$
\vec{h}_{1}\left(\epsilon+P_{\gamma \epsilon} A(y, s), y, s\right) \sqrt{1+\left|\nabla P_{\gamma \epsilon} A(y, s)\right|^{2}}=\frac{\partial P_{\gamma \epsilon} A(y, s)}{\partial s} \vec{g}(y, s),
$$




$$
\vec{h}_{2}\left(\epsilon+P_{\gamma \epsilon} A(y, s), y, s\right) \sqrt{1+\left|\nabla P_{\gamma \epsilon} A(y, s)\right|^{2}}=\frac{\partial \vec{g}(y, s)}{\partial s} .
$$

We may therefore conclude that,

$$
\frac{\partial S^{\epsilon}(\vec{f})}{\partial t}\left(x_{0}, x, t\right)=-\frac{\partial}{\partial x_{0}} S^{\epsilon}\left(\vec{h}_{1}\right)\left(x_{0}, x, t\right)+S^{\epsilon}\left(\vec{h}_{2}\right)\left(x_{0}, x, t\right) .
$$

We are interested in

$$
S_{t}^{\epsilon, \pm \delta}\left(\left\langle\frac{\partial S^{\epsilon}(\vec{f})}{\partial t}, N_{t}^{\epsilon, \delta}\right\rangle\right)(P, t)=\int_{\partial \Omega_{t}^{\epsilon, \pm \delta}} \frac{\left\langle\frac{\partial S^{\epsilon}(\vec{f})}{\partial t}, N_{t}^{\epsilon, \delta}\right\rangle(Q, t)}{w_{n}(2-n)|P-Q|^{n-2}} d \sigma_{t}^{\epsilon, \pm \delta}(Q)
$$

for $P \in \partial \Omega_{t}^{\epsilon, \pm \delta}$. But,

$$
\left\langle\frac{\partial S^{\epsilon}(\vec{f})}{\partial t}, N_{t}^{\epsilon, \delta}\right\rangle=-\left\langle\frac{\partial}{\partial x_{0}} S^{\epsilon}\left(\vec{h}_{1}\right), N_{t}^{\epsilon, \delta}\right\rangle+\left\langle S^{\epsilon}\left(\vec{h}_{2}\right), N_{t}^{\epsilon, \delta}\right\rangle .
$$

We need to examine the first term on the right hand side a bit more closely. Using the trace result of Lemma 2.5 we have

$$
\begin{aligned}
& \lim _{\delta \rightarrow 0^{+}}\left(\frac{\partial}{\partial x_{0}} S^{\epsilon}\left(\vec{h}_{1}\right)^{j}\right) \circ \rho_{\epsilon, \pm \delta} N_{t}^{\epsilon, \delta, j} \\
= & \pm \frac{1}{2} N_{t}^{\epsilon, j}\left\{h_{1}^{j}-N_{t}^{\epsilon, j}\left\langle\vec{h}_{1}, N_{t}^{\epsilon}\right\rangle\right\} N_{t}^{\epsilon, j}+K_{j, 0}\left(\vec{h}_{1}\right) N_{t}^{\epsilon, j} .
\end{aligned}
$$

I.e.,

$$
\left.\lim _{\delta \rightarrow 0^{+}}\left\langle\frac{\partial}{\partial x_{0}} S^{\epsilon}\left(\vec{h}_{1}\right)\right) \circ \rho_{\epsilon, \pm \delta}, N_{t}^{\epsilon, \delta}\right\rangle=\sum_{j=0}^{n-1} K_{j, 0}\left(\vec{h}_{1}\right) N_{t, j}^{\epsilon}
$$

and we may therefore conclude that $\left\langle\frac{\partial S^{\epsilon}(\vec{f})}{\partial t}, N_{t}^{\epsilon, \delta}\right\rangle$ is a continuous function in all of $\mathbb{R}^{n+1}$. The conclusion in the statement of the lemma now follows from the smoothness of $\partial \Omega^{\epsilon}$ and the continuity of $S_{t}^{\epsilon}$.

8. Inequalities based on Rellich identities. In this section we focus on inequalities based on Rellich identities. Let $\Omega^{+}:=\Omega, \Omega^{-}:=\mathbb{R}^{n+1} \backslash \Omega$. We also define $\vec{u}_{+}:=\left.S \vec{f}\right|_{\Omega^{+}}, \vec{u}_{-}:=\left.S \vec{f}\right|_{\Omega^{-}}, p_{+}:=\left.p\right|_{\Omega_{+}}, p_{-}:=\left.p\right|_{\Omega_{-}}$. Then the following identities are valid,

$$
\begin{gathered}
\int_{-\infty}^{\infty} \int_{\partial \Omega_{s}}\left\langle\alpha, N_{s}\right\rangle\left|\nabla \vec{u}_{ \pm}\right|^{2} d \sigma_{s} d s=2 \int_{-\infty}^{\infty} \int_{\partial \Omega_{s}} \alpha^{j}\left(\frac{\partial u_{ \pm}}{\partial x_{j}}\right)^{r}\left(\frac{\partial u_{ \pm}}{\partial \nu}\right)^{r} d \sigma_{s} d s \\
\pm \int_{-\infty}^{\infty} \int_{\Omega_{s}^{ \pm}}(\operatorname{div} \vec{\alpha})|\nabla \vec{u}|^{2} d x d s \mp 2 \int_{-\infty}^{\infty} \int_{\Omega_{s}^{ \pm}}\left[\frac{\partial \alpha^{j}}{\partial x_{k}} \frac{\partial u^{r}}{\partial x_{j}} \frac{\partial u^{r}}{\partial x_{k}}-\frac{\partial \alpha^{j}}{\partial x_{r}} \frac{\partial u^{r}}{\partial x_{j}} p\right] d x d s \\
\mp 2 \int_{-\infty}^{\infty} \int_{\Omega_{s}^{ \pm}} \alpha^{j} \frac{\partial u^{r}}{\partial x_{j}} \frac{\partial u^{r}}{\partial s} d x d s
\end{gathered}
$$




$$
\begin{gathered}
\int_{-\infty}^{\infty} \int_{\partial \Omega_{s}}\left\langle\alpha, N_{s}\right\rangle\left|\nabla \vec{u}_{ \pm}\right|^{2} d \sigma_{s} d s=2 \int_{-\infty}^{\infty} \int_{\partial \Omega_{s}} \alpha^{j} \frac{\partial u_{ \pm}^{r}}{\partial x_{k}}\left(\frac{\partial u_{ \pm}^{r}}{\partial x_{k}} N_{s}^{j}-\frac{\partial u_{ \pm}^{r}}{\partial x_{j}} N_{s}^{k}\right) d \sigma_{s} d s \\
+2 \int_{-\infty}^{\infty} \int_{\partial \Omega_{s}} \alpha^{j} p_{ \pm}\left(\frac{\partial u_{ \pm}^{r}}{\partial x_{k}} N_{s}^{j}-\frac{\partial u_{ \pm}^{r}}{\partial x_{j}} N_{s}^{k}\right) d \sigma_{s} d s \\
\mp \int_{-\infty}^{\infty} \int_{\Omega_{s}^{ \pm}}(\operatorname{div} \vec{\alpha})|\nabla \vec{u}|^{2} d x d s \pm 2 \int_{-\infty}^{\infty} \int\left[\frac{\partial \alpha^{j}}{\partial x_{k}} \frac{\partial u^{r}}{\partial x_{j}} \frac{\partial u^{r}}{\partial x_{k}}-\frac{\partial \alpha^{j}}{\partial x_{r}} \frac{\partial u^{r}}{\partial x_{j}} p\right] d x d s \\
\pm 2 \int_{-\infty}^{\infty} \int_{\Omega_{s}^{ \pm}} \alpha^{j} \frac{\partial u^{r}}{\partial x_{j}} \frac{\partial u^{r}}{\partial s} d x d s .
\end{gathered}
$$

To simplify notation we introduce the following,

Definition. For each $f \in L^{2}\left(\partial \Omega^{\epsilon}\right)$ we let $\vec{u}=S \vec{f}$ be the singular layer potential with $\Omega^{\epsilon}$ as the domain of reference. We define

$$
A(\vec{u}):=\int_{-\infty}^{\infty} \int_{\partial \Omega_{t}^{\epsilon}}\left|S_{t}^{\epsilon}\left(\left\langle\frac{\partial \vec{u}}{\partial t}, N_{t}^{\epsilon}\right\rangle\right)\right|^{2} d \sigma_{t}^{\epsilon} d t .
$$

The main results are Lemma 8.1 and Lemma 8.2 below. $\nabla_{T}$ denotes the naturally defined tangential derivatives.

LEMMA 8.1. Let in the following $(\vec{u}, p)=(S \vec{f}, p)$ be the singular layer potential and pressure defined with $\Omega^{\epsilon}$ as the domain of reference and let $\vec{f} \in L^{2}\left(\partial \Omega^{\epsilon}\right)$. Then the following inequalities are valid with a constant $C$ independent of $\vec{f}$ and $\epsilon$.

(i) $\quad\left\|\nabla \vec{u}_{ \pm}\right\|_{L^{2}\left(\partial \Omega^{\epsilon}\right)}^{2}+\left\|p_{ \pm}\right\|_{L^{2}\left(\partial \Omega^{\epsilon}\right)}^{2}$

$$
\leq C\left[\left\|\nabla_{T} \vec{u}\right\|_{L^{2}\left(\partial \Omega^{\epsilon}\right)}^{2}+A(\vec{u})+\left|\sum_{r=0}^{n-1} \int_{\Omega^{\epsilon}, \pm} \frac{\partial u^{r}}{\partial x_{0}} \frac{\partial u^{r}}{\partial t} d X d t\right|\right]
$$

(ii) $\left\|\nabla_{T} \vec{u}\right\|_{L^{2}\left(\partial \Omega^{\epsilon}\right)}^{2}+A(\vec{u}) \leq C\left[\left\|\frac{\partial \vec{u}_{ \pm}}{\partial \nu}\right\|_{L^{2}\left(\partial \Omega^{\epsilon}\right)}^{2}+\left|\sum_{r=0}^{n-1} \int_{\Omega^{\epsilon, \pm}} \frac{\partial u^{r}}{\partial x_{0}} \frac{\partial u^{r}}{\partial t} d X d t\right|\right]$.

Proof. Using the second Rellich identity we have

$$
\begin{aligned}
\left\|\nabla \vec{u}_{ \pm}\right\|_{L^{2}\left(\partial \Omega^{\epsilon}\right)}^{2} \leq C & {\left[\left\|\nabla \vec{u}_{ \pm}\right\|_{L^{2}\left(\partial \Omega^{\epsilon}\right)}\left\|\nabla_{T} \vec{u}\right\|_{L^{2}\left(\partial \Omega^{\epsilon}\right)}\right.} \\
& \left.+\left\|p_{ \pm}\right\|_{L^{2}\left(\partial \Omega^{\epsilon}\right)}\left\|\nabla_{T} \vec{u}\right\|_{L^{2}\left(\partial \Omega^{\epsilon}\right)}+\left|\sum_{r=0}^{n-1} \int_{\Omega^{\epsilon, \pm}} \frac{\partial u^{r}}{\partial x_{0}} \frac{\partial u^{r}}{\partial t} d x d t\right|\right] .
\end{aligned}
$$


Using part (i),(ii) of Lemma 7.2 as well as Lemma 7.3 we have,

$$
\left\|\nabla \vec{u}_{ \pm}\right\|_{L^{2}\left(\partial \Omega^{\epsilon}\right)}^{2}+\left\|p_{ \pm}\right\|_{L^{2}\left(\partial \Omega^{\epsilon}\right)}^{2} \leq C\left[\left\|\nabla \vec{u}_{ \pm}\right\|_{L^{2}\left(\partial \Omega^{\epsilon}\right)}^{2}+A(\vec{u})\right] .
$$

Combining these estimates we get the conclusion in (i). To prove the second inequality we use the first Rellich identity. This gives us,

$$
\left\|\nabla \vec{u}_{ \pm}\right\|_{L^{2}\left(\partial \Omega^{\epsilon}\right)}^{2} \leq C\left[\left\|\nabla \vec{u}_{ \pm}\right\|_{L^{2}\left(\partial \Omega^{\epsilon}\right)}\left\|\frac{\partial \vec{u}_{ \pm}}{\partial \nu}\right\|_{L^{2}\left(\partial \Omega^{\epsilon}\right)}+\left|\sum_{r=0}^{n-1} \int_{\Omega^{\epsilon, \pm}} \frac{\partial u^{r}}{\partial x_{0}} \frac{\partial u^{r}}{\partial t} d x d t\right|\right]
$$

Using Lemma 7.2 part (iii) we have

$$
\left\|\nabla \vec{u}_{ \pm}\right\|_{L^{2}\left(\partial \Omega^{\epsilon}\right)}^{2}+A(\vec{u}) \leq C\left[\left\|p_{ \pm}\right\|_{L^{2}\left(\partial \Omega^{\epsilon}\right)}^{2}+\left\|\nabla \vec{u}_{ \pm}\right\|_{L^{2}\left(\partial \Omega^{\epsilon}\right)}^{2}\right] .
$$

As furthermore,

$$
\left\|p_{ \pm}\right\|_{L^{2}\left(\partial \Omega^{\epsilon}\right)}^{2} \leq\left\|\frac{\partial \vec{u}_{ \pm}}{\partial \nu}\right\|_{L^{2}\left(\partial \Omega^{\epsilon}\right)}^{2}+\left\|\nabla \vec{u}_{ \pm}\right\|_{L^{2}\left(\partial \Omega^{\epsilon}\right)}^{2}
$$

we get the following,

$$
\begin{gathered}
\left\|\nabla \vec{u}_{ \pm}\right\|_{L^{2}\left(\partial \Omega^{\epsilon}\right)}^{2}+A(\vec{u}) \leq C\left(\left\|\frac{\partial \vec{u}_{ \pm}}{\partial \nu}\right\|_{L^{2}\left(\partial \Omega^{\epsilon}\right)}^{2}+\left\|\nabla \vec{u}_{ \pm}\right\|_{L^{2}\left(\partial \Omega^{\epsilon}\right)}^{2}\right) \\
\leq C\left[\left\|\frac{\partial \vec{u}_{ \pm}}{\partial \nu}\right\|_{L^{2}\left(\partial \Omega^{\epsilon}\right)}\left\|\nabla \vec{u}_{ \pm}\right\|_{L^{2}\left(\partial \Omega^{\epsilon}\right)}+\left\|\frac{\partial \vec{u}_{ \pm}}{\partial \nu}\right\|_{L^{2}\left(\partial \Omega^{\epsilon}\right)}+\left|\sum_{r=0}^{n-1} \int_{\Omega^{\epsilon, \pm}} \frac{\partial u^{r}}{\partial x_{0}} \frac{\partial u^{r}}{\partial t} d x d t\right|\right] .
\end{gathered}
$$

From this we get (ii).

LEMMA 8.2. Let in the following $(\vec{u}, p)=(S \vec{f}, p)$ be the single layer potential and the pressure defined with $\Omega$ as the domain of reference and let $\vec{f} \in L^{2}(\partial \Omega)$. Then the following inequalities are valid with a constant $C$ independent of $\vec{f}$ and $\gamma$ if $\gamma<1 / 2$ and $\left\|\mathbb{D}_{n} A\right\|_{*} \leq \epsilon_{0} \leq \gamma^{8+d}$ is small enough.

$$
\begin{aligned}
& \left|\sum_{r=0}^{n-1} \int_{\Omega} \frac{\partial u^{r}}{\partial t} \frac{\partial u^{r}}{\partial x_{0}} d X d t\right| \leq C_{\beta}\left[\gamma\|\vec{f}\|_{2}^{2}+\|\vec{f}\|_{2}\left[\left\|S_{b} \vec{f}\right\|_{L_{1,1 / 2}^{2}(\partial \Omega)}\left\|\frac{\partial \vec{u}}{\partial \nu}\right\|_{2}\right]^{1 / 2}\right] \\
& \int_{\mathbb{R}^{n}}\left|D_{1 / 2}^{t}(\vec{u} \circ \rho)(0, z)\right|^{2} d z \leq C_{\beta}\left[\gamma\|\vec{f}\|_{2}^{2}+\|\vec{f}\|_{2}\left[\left\|S_{b} \vec{f}\right\|_{L_{1,1 / 2}^{2}(\partial \Omega)}\left\|\frac{\partial \vec{u}}{\partial \nu}\right\|_{2}\right]^{1 / 2}\right] .
\end{aligned}
$$

Proof.

$$
\int_{\Omega} \frac{\partial u^{r}}{\partial t} \frac{\partial u^{r}}{\partial x_{0}} d X d t=\int_{0}^{\infty} \int_{\mathbb{R}^{n}}\left(u_{x_{0}}^{r} \circ \rho\right)\left(u_{t}^{r} \circ \rho\right)\left(1+\frac{\partial P_{\gamma \lambda} A}{\partial \lambda}\right) d z d \lambda
$$

Using that

$$
\left(u^{r} \circ \rho\right)_{t}=\left(u_{x_{0}}^{r} \circ \rho\right) \frac{\partial P_{\gamma \lambda} A}{\partial t}+u_{t}^{r} \circ \rho
$$


we have,

$$
\begin{aligned}
& \int_{\Omega} \frac{\partial u^{r}}{\partial t} \frac{\partial u^{r}}{\partial x_{0}} d X d t=\int_{0}^{\infty} \int_{\mathbb{R}^{n}}\left(u_{x_{0}}^{r} \circ \rho\right)\left(u_{t}^{r} \circ \rho\right) \frac{\partial P_{\gamma \lambda} A}{\partial \lambda} d z d \lambda \\
- & \int_{0}^{\infty} \int_{\mathbb{R}^{n}}\left(u_{x_{0}}^{r} \circ \rho\right)^{2} \frac{\partial P_{\gamma \lambda} A}{\partial t} d z d \lambda+\int_{0}^{\infty} \int_{\mathbb{R}^{n}}\left(u_{x_{0}}^{r} \circ \rho\right)\left(u^{r} \circ \rho\right)_{t} d z d \lambda \\
= & I_{1}+I_{2}+I_{3}
\end{aligned}
$$

Integrating by parts w.r.t $\lambda$ in $I_{2}$ we have

$$
\begin{gathered}
I_{2}=\int_{0}^{\infty} \int_{\mathbb{R}^{n}} 2\left(u_{x_{0}}^{r} \circ \rho\right)\left(u_{x_{0}}^{r} \circ \rho\right)_{\lambda} \frac{\partial P_{\gamma \lambda} A}{\partial t} \lambda d z d \lambda+\int_{0}^{\infty} \int_{\mathbb{R}^{n}}\left(u_{x_{0}}^{r} \circ \rho\right)^{2} \frac{\partial^{2} P_{\gamma \lambda} A}{\partial \lambda \partial t} \lambda d z d \lambda \\
=\text { partial integration w.r.t t in the second term }= \\
\int_{0}^{\infty} \int_{\mathbb{R}^{n}} 2\left(u_{x_{0}}^{r} \circ \rho\right)\left(u_{x_{0} x_{0}}^{r} \circ \rho\right)\left(\frac{\partial P_{\gamma \lambda} A}{\partial t}\right) \lambda d z d \lambda-\int_{0}^{\infty} \int_{\mathbb{R}^{n}} 2\left(u_{x_{0}}^{r} \circ \rho\right)\left(u_{x_{0} t}^{r} \circ \rho\right) \frac{\partial P_{\gamma \lambda} A}{\partial \lambda} \lambda d z d \lambda
\end{gathered}
$$

Distributing the $t$-derivative in the term $I_{3}$ we have proved the following identity,

$$
\begin{aligned}
\int_{\Omega} \frac{\partial u^{r}}{\partial t} \frac{\partial u^{r}}{\partial x_{0}} d X d t & =\int_{0}^{\infty} \int_{\mathbb{R}^{n}}\left(u_{x_{0}}^{r} \circ \rho\right)\left(u_{t}^{r} \circ \rho\right) \frac{\partial P_{\gamma \lambda} A}{\partial \lambda} d z d \lambda \\
& +\int_{0}^{\infty} \int_{\mathbb{R}^{n}} 2\left(u_{x_{0}}^{r} \circ \rho\right)\left(u_{x_{0} x_{0}}^{r} \circ \rho\right)\left(\frac{\partial P_{\gamma \lambda} A}{\partial t}\right) \lambda d z d \lambda \\
& -\int_{0}^{\infty} \int_{\mathbb{R}^{n}}^{\infty} 2\left(u_{x_{0}}^{r} \circ \rho\right)\left(u_{x_{0} t}^{r} \circ \rho\right) \frac{\partial P_{\gamma \lambda} A}{\partial \lambda} \lambda d z d \lambda \\
& +c \int_{0}^{\infty} \int_{\mathbb{R}^{n}} H D_{3 / 4}^{t}\left(u^{r} \circ \rho\right) D_{1 / 4}^{t}\left(u_{x_{0}}^{r} \circ \rho\right) d z d \lambda .
\end{aligned}
$$

I.e,

$$
\left|\int_{\Omega} \frac{\partial u^{r}}{\partial t} \frac{\partial u^{r}}{\partial x_{0}} d X d t\right|
$$

can be estimated with the following sum of products.

$$
\begin{aligned}
& \left(\int_{0}^{\infty} \int_{\mathbb{R}^{n}}\left(u_{x_{0}}^{r} \circ \rho\right)^{2}\left(\frac{\partial P_{\gamma \lambda} A}{\partial \lambda}\right)^{2} \frac{d z d \lambda}{\lambda}\right)^{1 / 2} \times\left(\int_{0}^{\infty} \int_{\mathbb{R}^{n}}\left(\left(\Delta u^{r} \circ \rho\right)+\left(p_{x_{r}} \circ \rho\right)\right)^{2} \lambda d z d \lambda\right)^{1 / 2} \\
& \quad+\left(\int_{0}^{\infty} \int_{\mathbb{R}^{n}}\left(u_{x_{0}}^{r} \circ \rho\right)^{2}\left(\frac{\partial P_{\gamma \lambda} A}{\partial t}\right)^{2} \lambda d z d \lambda\right)^{1 / 2} \times\left(\int_{0}^{\infty} \int_{\mathbb{R}^{n}}\left(u_{x_{0} x_{0}}^{r} \circ \rho\right)^{2} \lambda d z d \lambda\right)^{1 / 2}
\end{aligned}
$$




$$
\begin{aligned}
& +\left(\int_{0}^{\infty} \int_{\mathbb{R}^{n}}\left(u_{x_{0}}^{r} \circ \rho\right)^{2}\left(\frac{\partial P_{\gamma \lambda} A}{\partial \lambda}\right)^{2} \frac{d z d \lambda}{\lambda}\right)^{1 / 2} \times\left(\int_{0}^{\infty} \int_{\mathbb{R}^{n}}\left(u_{x_{0} t}^{r} \circ \rho\right)^{2} \lambda^{3} d z d \lambda\right)^{1 / 2} \\
& +\left(\int_{0}^{\infty} \int_{\mathbb{R}^{n}}\left|D_{1 / 4}^{t}\left(u_{x_{j}}^{r} \circ \rho\right)\right|^{2} d z d \lambda\right)^{1 / 2} \times\left(\int_{0}^{\infty} \int_{\mathbb{R}^{n}}\left|D_{3 / 4}^{t}\left(u^{r} \circ \rho\right)\right|^{2} d z d \lambda\right)^{1 / 2} .
\end{aligned}
$$

Using Lemma 1.1, Lemma 2.3, Lemma 2.4, Theorem 3.1 in an by now familar manner we may conclude that,

$$
\begin{gathered}
\left|\int_{\Omega} \frac{\partial u^{r}}{\partial t} \frac{\partial u^{r}}{\partial x_{0}} d X d t\right| \lesssim \gamma\|\vec{f}\|_{2}^{2}+\left(\int_{0}^{\infty} \int_{\mathbb{R}^{n}}\left|D_{1 / 4}^{t}\left(u_{x_{j}}^{r} \circ \rho\right)\right|^{2} d z d \lambda\right)^{1 / 2} \\
\times\left(\int_{0}^{\infty} \int_{\mathbb{R}^{n}}\left|D_{3 / 4}^{t}\left(u^{r} \circ \rho\right)\right|^{2} d z d \lambda\right)^{1 / 2} .
\end{gathered}
$$

Applying Theorem 5.1 completes the proof.

We now manipulate the second quantity in the lemma.

$$
\begin{aligned}
\int_{\mathbb{R}^{n}}\left|D_{1 / 2}^{t}\left(u^{r} \circ \rho\right)(0, z)\right|^{2} d z= & -\int_{0}^{\infty} \int_{\mathbb{R}^{n}} \frac{\partial}{\partial \lambda}\left|D_{1 / 2}^{t}\left(u^{r} \circ \rho\right)(\lambda, z)\right|^{2} d z d \lambda \\
= & -2 \int_{0}^{\infty} \int_{\mathbb{R}^{n}} D_{1 / 2}^{t}\left(u^{r} \circ \rho\right) D_{1 / 2}^{t}\left(u_{x_{0}}^{r} \circ \rho\right) d z d \lambda \\
& -2 \int_{0}^{\infty} \int_{\mathbb{R}^{n}} D_{1 / 2}^{t}\left(u^{r} \circ \rho\right) D_{1 / 2}^{t}\left[\left(u_{x_{0}}^{r} \circ \rho\right) \frac{\partial P_{\gamma \lambda} A}{\partial \lambda}\right] d z d \lambda,
\end{aligned}
$$

where we have used that

$$
\left(u^{r} \circ \rho\right)_{\lambda}=\left(u_{x_{0}}^{r} \circ \rho\right)\left(1+\frac{\partial P_{\gamma \lambda} A}{\partial \lambda}\right) .
$$

Using the self-adjointness and the fact that $D_{1 / 2}^{t} * D_{1 / 2}^{t}=c H \frac{\partial}{\partial t}$ we have

$$
\begin{aligned}
\int_{\mathbb{R}^{n}}\left|D_{1 / 2}^{t}\left(u^{r} \circ \rho\right)(0, z)\right|^{2} d z= & -2 \int_{0}^{\infty} \int_{\mathbb{R}^{n}} D_{3 / 4}^{t}\left(u^{r} \circ \rho\right) D_{1 / 4}^{t}\left(u_{x_{0}}^{r} \circ \rho\right) d z d \lambda \\
& -2 c \int_{0}^{\infty} \int_{\mathbb{R}^{n}} H\left(u^{r} \circ \rho\right)_{t}\left(u_{x_{0}}^{r} \circ \rho\right) \frac{\partial P_{\gamma \lambda} A}{\partial \lambda} d z d \lambda .
\end{aligned}
$$

I.e,

$$
\begin{aligned}
& \int_{\mathbb{R}^{n}}\left|D_{1 / 2}^{t}\left(u^{r} \circ \rho\right)(0, z)\right|^{2} d z \\
\lesssim & \left(\int_{0}^{\infty} \int_{\mathbb{R}^{n}}\left|D_{1 / 4}^{t}\left(u_{x_{j}}^{r} \circ \rho\right)\right|^{2} d z d \lambda\right)^{1 / 2} \times\left(\int_{0}^{\infty} \int_{\mathbb{R}^{n}}\left|D_{3 / 4}^{t}\left(u^{r} \circ \rho\right)\right|^{2} d z d \lambda\right)^{1 / 2}
\end{aligned}
$$




$$
+\left(\int_{0}^{\infty} \int_{\mathbb{R}^{n}}\left(u_{x_{0}}^{r} \circ \rho\right)^{2}\left(\frac{\partial P_{\gamma \lambda} A}{\partial \lambda}\right)^{2} \frac{d z d \lambda}{\lambda}\right)^{1 / 2} \times\left(\int_{0}^{\infty} \int_{\mathbb{R}^{n}}\left|\left(u^{r} \circ \rho\right)_{t}\right|^{2} \lambda d z d \lambda\right)^{1 / 2} .
$$

But

$$
\left(u^{r} \circ \rho\right)_{t}=\left(u_{x_{0}}^{r} \circ \rho\right) \frac{\partial P_{\gamma \lambda} A}{\partial t}+u_{t}^{r} \circ \rho=\left(u_{x_{0}}^{r} \circ \rho\right) \frac{\partial P_{\gamma \lambda} A}{\partial t}+\left(\Delta u^{r}\right) \circ \rho+p_{x_{r}} \circ \rho .
$$

I.e,

$$
\begin{aligned}
& \int_{\mathbb{R}^{n}}\left|D_{1 / 2}^{t}\left(u^{r} \circ \rho\right)(0, z)\right|^{2} d z \\
\lesssim & \left(\int_{0}^{\infty} \int_{\mathbb{R}^{n}}\left|D_{1 / 4}^{t}\left(u_{x_{j}}^{r} \circ \rho\right)\right|^{2} d z d \lambda\right)^{1 / 2} \times\left(\int_{0}^{\infty} \int_{\mathbb{R}^{n}}\left|D_{3 / 4}^{t}\left(u^{r} \circ \rho\right)\right|^{2} d z d \lambda\right)^{1 / 2} \\
+ & \left(\int_{0}^{\infty} \int_{\mathbb{R}^{n}}\left(u_{x_{0}}^{r} \circ \rho\right)^{2}\left(\frac{\partial P_{\gamma \lambda} A}{\partial \lambda}\right)^{2} \frac{d z d \lambda}{\lambda}\right)^{1 / 2} \times\left(\int_{0}^{\infty} \int_{\mathbb{R}^{n}}\left|\left(\left(\Delta u^{r}\right) \circ \rho\right)+\left(p_{x_{r}} \circ \rho\right)\right|^{2} \lambda d z d \lambda\right)^{1 / 2} \\
+ & \int_{0}^{\infty} \int_{\mathbb{R}^{n}}\left(u_{x_{0}}^{r} \circ \rho\right)^{2}\left(\frac{\partial P_{\gamma \lambda} A}{\partial \lambda}\right)^{2} \frac{d z d \lambda}{\lambda} .
\end{aligned}
$$

The estimates stated in the corollary now follow from Lemma 1.1,Lemma 2.3, Lemma 2.4, Theorem 3.1 and Theorem 5.1.

LEMMA 8.3. Let in the following $(\vec{u}, p)=(S \vec{f}, p)$ be the single layer potential and the pressure defined with $\Omega^{\epsilon}$ as the domain of reference and let $\vec{f} \in L^{2}\left(\partial \Omega^{\epsilon}\right)$. Then the following inequalities are valid with a constant $C$ independent of $\epsilon, \vec{f}$ and $\gamma$ if $\gamma<1 / 2$ and if $\left\|\mathbb{D}_{n} A\right\|_{*} \leq \epsilon_{0} \leq \gamma^{8+d}$ is small enough.

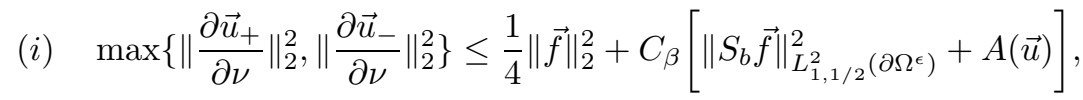

$$
\begin{aligned}
& \text { (ii) } \quad\left\|S_{b} \vec{f}\right\|_{L_{1,1 / 2}^{2}\left(\partial \Omega^{\epsilon}\right)}^{2}+A(\vec{u}) \leq \eta\|\vec{f}\|_{2}^{2}+C_{\beta} \min \left\{\left\|\frac{\partial \vec{u}_{+}}{\partial \nu}\right\|_{2}^{2},\left\|\frac{\partial \vec{u}_{-}}{\partial \nu}\right\|_{2}^{2}\right\},
\end{aligned}
$$

where $\eta \rightarrow 0$ as $\left\|\mathbb{D}_{n} A\right\|_{*} \rightarrow 0$.

Proof. Using Lemma 8.1 and 8.2 we have,

$$
\begin{gathered}
\left\|\frac{\partial \vec{u}_{ \pm}}{\partial \nu}\right\|_{2}^{2} \leq C\left[\left\|S_{b} \vec{f}\right\|_{L_{1,1 / 2}^{2}\left(\partial \Omega^{\epsilon}\right)}^{2}+A(\vec{u})+\left|\sum_{r=0}^{n-1} \int_{\Omega^{\epsilon, \pm}} \frac{\partial u^{r}}{\partial x_{0}} \frac{\partial u^{r}}{\partial t} d x d t\right|\right] \\
\leq C\left[\left\|S_{b} \vec{f}\right\|_{L_{1,1 / 2}^{2}\left(\partial \Omega^{\epsilon}\right)}^{2}+A(\vec{u})+\gamma\|\vec{f}\|_{2}^{2}+\left[\left\|S_{b} \vec{f}\right\|_{L_{1,1 / 2}^{2}\left(\partial \Omega^{\epsilon}\right)}\left\|\frac{\partial \vec{u}_{ \pm}}{\partial \nu}\right\|_{2}\right]^{1 / 2}\|\vec{f}\|_{2}\right]
\end{gathered}
$$

This gives the first inequality after some simple manipulations.

Again using Lemma 8.1 and Lemma 8.2 we have

$$
\begin{aligned}
& \left\|S_{b} \vec{f}\right\|_{L_{1,1 / 2}^{2}\left(\partial \Omega^{\epsilon}\right)}^{2}+A(\vec{u}) \\
\leq & C\left[\min \left\{\left\|\frac{\partial \vec{u}_{+}}{\partial \nu}\right\|_{2},\left\|\frac{\partial \vec{u}}{\partial \nu}\right\|_{2}\right\}+\gamma\|\vec{f}\|_{2}^{2}+\|\vec{f}\|_{2}\left[\left\|S_{b} \vec{f}\right\|_{L_{1,1 / 2}^{2}(\partial \Omega)}\left\|\frac{\partial \vec{u}_{ \pm}}{\partial \nu}\right\|_{2}\right]^{1 / 2}\right] .
\end{aligned}
$$


Again this gives the inequality after some simple manipulations.

9. Invertability of the single layer potential and existence results. We will now prove the existence part of Theorem A-C. First we will state an appropriate definition of weak solutions for the Dirichlet problem. Let as usual $\Omega^{\epsilon}=\left\{\left(x_{0}, x, t\right)\right.$ : $\left.x_{0}>\epsilon+P_{\gamma \epsilon} A(x, t)\right\}$. Recall the definition of the regularity space $R\left(\partial \Omega^{\epsilon}\right)$.

$$
R\left(\partial \Omega^{\epsilon}\right)=\left\{\vec{g} \in L_{1,1 / 2}^{2}\left(\partial \Omega^{\epsilon}\right) \cap L_{N}^{2}\left(\partial \Omega^{\epsilon}\right),\left\langle\frac{\partial \vec{g}}{\partial t}, N_{t}^{\epsilon}\right\rangle \in L^{2}\left(-\infty, \infty,\left[L_{1}^{2}\left(\partial \Omega_{t}^{\epsilon}\right)\right]^{*}\right)\right\},
$$

where $\left[L_{1}^{2}\left(\partial \Omega_{t}^{\epsilon}\right)\right]^{*}$ is the dual space of $L_{1}^{2}\left(\partial \Omega_{t}^{\epsilon}\right)$ and

$$
L_{N}^{2}\left(\partial \Omega^{\epsilon}\right)=\left\{\vec{f} \in L^{2}\left(\partial \Omega^{\epsilon}\right): \int_{\partial \Omega_{t}^{\epsilon}}\left\langle\vec{f}, N_{t}\right\rangle d \sigma_{t}^{\epsilon}=0 \quad \text { for almost all } \mathrm{t}\right\} .
$$

Recall that for each $t, L_{1}^{2}\left(\partial \Omega_{t}^{\epsilon}\right)$ consists of functions with distributional derivatives in $x$ satisfying $\|f\|_{L_{1}^{2}\left(\partial \Omega_{t}^{\epsilon}\right)}<\infty$, where $\|f\|_{L_{1}^{2}\left(\partial \Omega_{t}^{\epsilon}\right)}=\left\|\nabla_{x} \tilde{f}\right\|_{2}$ and $\tilde{f}=f \circ \pi^{-1}$, $\pi(A(x, t), x, t)=(x, t)$. The norm of an element $\vec{g} \in R\left(\partial \Omega^{\epsilon}\right)$ is defined as

$$
\|\vec{g}\|_{L_{1,1 / 2}^{2}\left(\partial \Omega^{\epsilon}\right)}+\left[\int_{-\infty}^{\infty}\left\|\left\langle\frac{\partial \vec{g}}{\partial t}, N_{t}^{\epsilon}\right\rangle\right\|_{\left[L_{1}^{2}\left(\partial \Omega_{t}^{\epsilon}\right)\right]^{*}}^{2} d t\right]^{1 / 2}
$$

Definition 9.1. We call $(\vec{\phi}, q)$ a pair of admissible test functions in $\Omega^{\epsilon}$ if the following is true,

(i) $\vec{\phi} \in C^{\infty}\left(\overline{\Omega^{\epsilon}}\right), \quad q \in C^{\infty}\left(\overline{\Omega^{\epsilon}}\right)$

(ii) $\operatorname{div} \vec{\phi}=0$ in $\Omega^{\epsilon}$

(iii) $\vec{\phi}=\overrightarrow{0}$ on $\partial \Omega^{\epsilon} \quad$ in $R\left(\partial \Omega^{\epsilon}\right)$

(iv) For all $\alpha \in \mathbb{N}$ and all multiindices $\beta, \vec{\phi}$ satisfies the following decay estimate for $\left(|X|+|t|^{1 / 2}\right)$ sufficiently large : $\left|\partial_{t}^{\alpha} \partial_{X}^{\beta} \vec{\phi}(X, t)\right| \leq C(\alpha, \beta, \vec{\phi})\left(|X|+|t|^{1 / 2}\right)^{-n-2 \alpha-|\beta|}$

$(v)$ For almost every $t$ and every multiindex $\alpha$ there exists a constant $C$ such that for $|X|$ sufficiently large: $\quad\left|\partial_{x}^{\alpha} q(X, t)\right| \leq C(\alpha, q)|X|^{1-n-|\alpha|}$.

Definition 9.2. We say that $\vec{u}$ is a weak solution to the problem

$$
\begin{aligned}
& \frac{\partial \vec{u}}{\partial t}=\Delta \vec{u}-\nabla p \quad \text { in } \quad \Omega=\left\{\left(x_{0}, x, t\right): x_{0}>A(x, t)\right\}, \\
& \operatorname{div} \vec{u}=0 \text { in } \Omega \\
& \vec{u}=\vec{f} \in L_{N}^{2}(\partial \Omega) \quad \text { a.e on } \partial \Omega
\end{aligned}
$$

if and only if it is true that for for almost every $t, \tilde{N}_{*, e}(\vec{u}) \in L^{2}\left(\partial \Omega_{t}\right)$, and for almost every $\left(x_{0}, x\right) \in \partial \Omega_{t}$ w.r.t $d \sigma_{t}$

$$
\lim _{\left(y_{0}, y, t\right) \rightarrow\left(x_{0}, x, t\right)} \vec{u}\left(y_{0}, y, t\right)=\vec{f}\left(x_{0}, x, t\right) .
$$


Furthermore, for every $\epsilon>0$ and every pair of admissible test functions $(\vec{\phi}, q)$ in $\Omega^{\epsilon}$ the following should be true,

$$
\int_{\Omega^{\epsilon}}\left\langle\vec{u}, \vec{\phi}_{s}+\Delta \vec{\phi}+\nabla q\right\rangle d Y d s=\int_{\partial \Omega^{\epsilon}}\left\langle\vec{u}, \frac{\partial \vec{\phi}}{\partial N_{s}^{\epsilon}}\right\rangle d \sigma_{s}^{\epsilon} d s+\int_{\partial \Omega^{\epsilon}}\left\langle\vec{u}, N_{s}^{\epsilon}\right\rangle q d \sigma_{s}^{\epsilon} d s .
$$

Let us for clarity prove that the double layer potential $\vec{u}=D \vec{f}$ is a weak solution. We want to prove that the difference

$$
\int_{\Omega^{\epsilon}}\left\langle\vec{u}, \vec{\phi}_{s}+\Delta \vec{\phi}+\nabla q\right\rangle d Y d s-\int_{\partial \Omega^{\epsilon}}\left\langle\vec{u}, \frac{\partial \vec{\phi}}{\partial N_{s}^{\epsilon}}\right\rangle d \sigma_{s}^{\epsilon} d s-\int_{\partial \Omega^{\epsilon}}\left\langle\vec{u}, N_{s}^{\epsilon}\right\rangle q d \sigma_{s}^{\epsilon} d s
$$

is zero. As $\langle\vec{u}, \nabla q\rangle=\operatorname{div}(\vec{u} q)$ we have using decay properties of $\vec{u}$ and $q$ that

$$
\int_{\Omega^{\epsilon}}\langle\vec{u}, \nabla q\rangle d Y d s-\int_{\partial \Omega^{\epsilon}}\left\langle\vec{u}, N_{s}^{\epsilon}\right\rangle q d \sigma_{s}^{\epsilon} d s=0 .
$$

Therefore we have to focus on the difference,

$$
\int_{\Omega^{\epsilon}}\left\langle\vec{u}, \vec{\phi}_{s}+\Delta \vec{\phi}\right\rangle d Y d s-\int_{\partial \Omega^{\epsilon}}\left\langle\vec{u}, \frac{\partial \vec{\phi}}{\partial N_{s}^{\epsilon}}\right\rangle d \sigma_{s}^{\epsilon} d s
$$

which by integration by parts equals

$$
\int_{\Omega^{\epsilon}}\left\langle\vec{u}, \vec{\phi}_{s}\right\rangle+\langle\Delta \vec{u}, \vec{\phi}\rangle d Y d s
$$

Recall that the double layer by definition is,

$$
\begin{aligned}
\vec{u}(X, t)=D \vec{f}(X, t) & :=\int_{-\infty}^{t} \int_{\partial \Omega_{s}} \frac{\partial \Gamma}{\partial N_{s}(Q)}(X-Q, t-s) \vec{f}(Q, s) d \sigma_{s}(Q) d s \\
& -\int_{\partial \Omega_{t}} \frac{Q-X}{w_{n}|X-Q|^{n}}\left\langle\vec{f}(Q, t), N_{t}(Q)\right\rangle d \sigma_{t}(Q) .
\end{aligned}
$$

I.e., $\vec{u}=\vec{u}_{1}+\vec{u}_{2}$ with $\vec{u}_{2}$ harmonic. Therefore,

$$
\begin{aligned}
& \int_{\Omega^{\epsilon}}\left\langle\vec{u}, \vec{\phi}_{s}\right\rangle+\langle\Delta \vec{u}, \vec{\phi}\rangle d Y d s \\
= & \int_{\Omega^{\epsilon}}\left\langle\vec{u}_{1}, \vec{\phi}_{s}\right\rangle+\left\langle\Delta \vec{u}_{1}, \vec{\phi}\right\rangle+\left\langle\vec{u}_{2}, \vec{\phi}_{s}\right\rangle d Y d s \\
= & -\int_{\Omega^{\epsilon}}\left\langle\frac{\partial \vec{u}_{1}}{\partial s}-\Delta \vec{u}_{1}, \vec{\phi}_{s}\right\rangle+\int_{\Omega^{\epsilon}}\left\langle\vec{u}_{2}, \vec{\phi}_{s}\right\rangle d Y d s .
\end{aligned}
$$

As $\vec{u}_{1}$ essentially can be written as a sum of derivatives of single layer potentials we essentially have $\frac{\partial \vec{u}_{1}}{\partial s}-\Delta \vec{u}_{1}=\nabla p_{x_{k}}$ for some harmonic pressure $p$. Again using the 
divergence theorem and the fact that our test function $\vec{\phi}$ is zero on the boundary we have

$$
\int_{\Omega^{\epsilon}}\left\langle\frac{\partial \vec{u}_{1}}{\partial s}-\Delta \vec{u}_{1}, \vec{\phi}_{s}\right\rangle=0
$$

Left to prove is that,

$$
\int_{\Omega^{\epsilon}}\left\langle\vec{u}_{2}, \vec{\phi}_{s}\right\rangle d Y d s=0
$$

But $\vec{u}_{2}=\nabla S g$ for an appropriate $g$. Here $S g$ is the single layer potential associated to the Laplace operator. Therefore as $\vec{\phi}=\overrightarrow{0}$ in $R\left(\partial \Omega^{\epsilon}\right)$ we have,

$$
\int_{\Omega^{\epsilon}}\left\langle\vec{u}_{2}, \vec{\phi}_{s}\right\rangle d Y d s=\int_{\Omega^{\epsilon}}\left\langle\nabla S g, \vec{\phi}_{s}\right\rangle d Y d s=\int_{\partial \Omega^{\epsilon}}\left\langle\vec{\phi}_{s}, N_{s}\right\rangle S g d \sigma_{s} d s=0 .
$$

One also easily verifies that the double layer potential $D \vec{f}, \vec{f} \in L^{2}(\partial \Omega)$, has boundary values $(\vec{f} / 2+\tilde{K} \vec{f})$.

We will now prove the relevant invertibility results on the approximating domains $\Omega^{\epsilon}$ with $\epsilon>0$ small. In the case of the Dirichlet and Neumann problems we can then easily pass to the limiting domain ( see the remark after Theorem 9.2). Combining the inequalities of Lemma 8.3 we have that there exists a constant $C$ independent of $\vec{f} \in L^{2}\left(\partial \Omega^{\epsilon}\right)$ and $\epsilon$ such that if $\vec{u}=S \vec{f}=S^{\epsilon} \vec{f}$ then,

$$
\begin{gathered}
\|\vec{f}\|_{L^{2}\left(\partial \Omega^{\epsilon}\right)} \leq C \min \left\{\left\|\frac{\partial \vec{u}_{+}}{\partial \nu}\right\|_{L^{2}\left(\partial \Omega^{\epsilon}\right)},\left\|\frac{\partial \vec{u}_{-}}{\partial \nu}\right\|_{L^{2}\left(\partial \Omega^{\epsilon}\right)}\right\} \\
\|\vec{f}\|_{L^{2}\left(\partial \Omega^{\epsilon}\right)} \leq C\left[\|\vec{u}\|_{L_{1,1 / 2}^{2}\left(\partial \Omega^{\epsilon}\right)}+\left(\int_{-\infty}^{\infty} \int_{\partial \Omega_{t}^{\epsilon}}\left|S_{t}^{\epsilon}\left(\left\langle\frac{\partial \vec{u}}{\partial t}, N_{t}^{\epsilon}\right\rangle\right)\right|^{2} d \sigma_{t}^{\epsilon} d t\right)^{1 / 2}\right]
\end{gathered}
$$

Now assume $\vec{f} \in L^{2}\left(\partial \Omega^{\epsilon}\right)$. Then $\partial \vec{u}_{ \pm} / \partial \nu=\left( \pm I / 2+K_{\nu}\right) \vec{f}=\left( \pm I / 2+K_{\epsilon, \nu}\right) \vec{f}$ a.e on $\partial \Omega^{\epsilon}$ by Lemma 2.6 and 2.7 .

TheOREM 9.1. Let $\Omega^{\epsilon}=\left\{\left(x_{0}, x, t\right) \in \mathbb{R} \times \mathbb{R}^{n-1} \times \mathbb{R}: x_{0}>\epsilon+P_{\gamma \epsilon} A(x, t)\right\}$ where $\|A\|_{\text {comm }} \leq \beta<\infty$ and $\left\|D_{1 / 2}^{t} A\right\|_{*} \leq \epsilon_{0}<\infty$. If $\epsilon_{0}=\epsilon_{0}\left(\left\|\nabla_{x} A\right\|_{\infty}\right)$ is small enough then $\left( \pm \frac{1}{2} I+K_{\nu}\right)=\left( \pm \frac{1}{2} I+K_{\epsilon, \nu}\right)$ is an invertible operator from $L^{2}\left(\partial \Omega^{\epsilon}\right)$ to $L^{2}\left(\partial \Omega^{\epsilon}\right)$.

Proof. We want to prove that $\vec{f} \rightarrow\left(I / 2+K_{\nu}\right) \vec{f}$ is $1-1$ and onto $L^{2}\left(\partial \Omega^{\epsilon}\right)$. We know that the map is into $L^{2}\left(\partial \Omega^{\epsilon}\right)$. Let $T_{\alpha}(x, t)=\left(\left(I / 2+K_{\nu}^{\alpha}\right) \vec{f}\right) \circ \rho(0, x, t)$ where $K_{\nu}^{\alpha}$ is the operator we get if $A(x, t)$ is replaced with $\alpha A(x, t), \alpha \in[0,1]$. Then obviously $T_{\alpha}: L^{2}\left(\mathbb{R}^{n}\right) \rightarrow L^{2}\left(\mathbb{R}^{n}\right)$ with norm independent of $\alpha$ and $\|\vec{f} \circ \rho\|_{L^{2}\left(\mathbb{R}^{n}\right)} \leq C\left\|T_{\alpha} \vec{f}\right\|_{L^{2}\left(\mathbb{R}^{n}\right)}$, where $C$ is independent of $\alpha$. The last inequality is the inequality stated in (1). Using arguments similar to the ones needed in the proof of Theorem 2.1, i.e. the results on singular integrals of Hofmann[18], one can prove that $\frac{d T_{\alpha}}{d \alpha}: L^{2}\left(\mathbb{R}^{n}\right) \rightarrow L^{2}\left(\mathbb{R}^{n}\right)$. Invertibility now follows from the method of continuity as described in [23,p.150].

Theorem 9.2. Let $\Omega^{\epsilon}=\left\{\left(x_{0}, x, t\right) \in \mathbb{R} \times \mathbb{R}^{n-1} \times \mathbb{R}: x_{0}>\epsilon+P_{\gamma \epsilon} A(x, t)\right\}$ where $\|A\|_{\text {comm }} \leq \beta<\infty$ and $\left\|D_{1 / 2}^{t} A\right\|_{*} \leq \epsilon_{0}<\infty$. If $\epsilon_{0}=\epsilon_{0}\left(\left\|\nabla_{x} A\right\|_{\infty}\right)$ is small enough then $\left( \pm \frac{1}{2} I+\tilde{K}_{\epsilon, v}\right)=\left( \pm \frac{1}{2} I+\tilde{K}_{v}\right)$ is an invertible operator from $L^{2}\left(\partial \Omega^{\epsilon}\right)$ to $L_{N}^{2}\left(\partial \Omega^{\epsilon}\right)$. 
Proof. Note that if $(\vec{u}(X, t), p(X, t))$ solves the system of linearized Navier-Stokes in $\left\{(X, t): x_{0}>\epsilon+P_{\gamma \epsilon} A(x, t)\right\}$ then $(\vec{u}(X,-t), p(X,-t))$ solves the adjoint system in $\left\{(X, t): x_{0}>\epsilon+P_{\gamma \epsilon} A(x,-t)\right\}$. Obviously, $A(x,-t)$ has the same properties as $A(x, t)$. Let $\tilde{K}_{\nu}^{*}$ be the boundary operator associated to the adjoint single layer potential on $\left\{(X, t): x_{0}>\epsilon+P_{\gamma \epsilon} A(x,-t)\right\}$. Invertibility of $\pm \frac{1}{2} I+\tilde{K}_{\nu}^{*}$ then follows from Theorem 9.1. The invertibility of $\pm \frac{1}{2} I+\tilde{K}_{\nu}$ now follows by duality.

REMARK. That Theorem 9.1 and 9.2 remain valid with $\Omega^{\epsilon}$ replaced with $\Omega$ follows from a simple approximation argument.

TheOREM 9.3. Let $\Omega^{\epsilon}=\left\{\left(x_{0}, x, t\right) \in \mathbb{R} \times \mathbb{R}^{n-1} \times \mathbb{R}: x_{0}>\epsilon+P_{\gamma \epsilon} A(x, t)\right\}$ where $\|A\|_{\text {comm }} \leq \beta<\infty$ and $\left\|D_{1 / 2}^{t} A\right\|_{*} \leq \epsilon_{0}<\infty$. If $\epsilon_{0}=\epsilon_{0}\left(\left\|\nabla_{x} A\right\|_{\infty}\right)$ is small enough then the map $\vec{f} \rightarrow S^{\epsilon} \vec{f}=S \vec{f}$ from $L^{2}\left(\partial \Omega^{\epsilon}\right)$ to $R\left(\partial \Omega^{\epsilon}\right)$ is one-to-one and onto, i.e. invertible.

Proof. Let $\vec{f} \in L^{2}\left(\partial \Omega^{\epsilon}\right)$ and let $\vec{u}=S \vec{f}$. Consider $S_{t}$, the single layer potential operator associated to the Laplace operator on $\Omega_{t}^{\epsilon}$. Using elliptic theory we know that $S_{t}^{-1}$ exists and is continuous from $L_{1}^{2}\left(\partial \Omega_{t}^{\epsilon}\right)$ to $L^{2}\left(\partial \Omega_{t}^{\epsilon}\right)$. For $\psi \in L_{1}^{2}\left(\partial \Omega_{t}^{\epsilon}\right)$ we define the functional,

$$
\left\langle\frac{\partial \vec{u}}{\partial t}, N_{t}^{\epsilon}\right\rangle(\psi)=\int_{-\infty}^{\infty} \int_{\partial \Omega_{t}^{\epsilon}} S_{t}^{\epsilon}\left(\left\langle\frac{\partial \vec{u}}{\partial t}, N_{t}^{\epsilon}\right\rangle\right) S_{t}^{-1}(\psi) d \sigma_{t}^{\epsilon} d t .
$$

Using Lemma 7.2 and Lemma 7.3 it then follows that $\vec{u} \in R\left(\partial \Omega^{\epsilon}\right)$ and

$$
\|\vec{u}\|_{R\left(\partial \Omega^{\epsilon}\right)} \leq C\|\vec{f}\|_{L^{2}\left(\partial \Omega^{\epsilon}\right)} .
$$

Therefore the map $\vec{f} \rightarrow S \vec{f}$ is into $R\left(\partial \Omega^{\epsilon}\right)$. Using inequality (2) above we also have the opposite inequality. Furthermore, using the results of Section 7 we have,

$$
\begin{gathered}
\|\vec{u}\|_{L_{1,1 / 2}^{2}\left(\partial \Omega^{\epsilon}\right)}^{2}+\int_{-\infty}^{\infty}\left\|\left\langle\frac{\partial \vec{u}}{\partial t}, N_{t}^{\epsilon}\right\rangle\right\|_{\left[L_{1}^{2}\left(\partial \Omega_{t}^{\epsilon}\right)\right]^{*}}^{2} d t \sim \\
\|\vec{u}\|_{L_{1,1 / 2}^{2}\left(\partial \Omega^{\epsilon}\right)}^{2}+\int_{-\infty}^{\infty} \int_{\partial \Omega_{t}^{\epsilon}}\left|S_{t}^{\epsilon}\left(\left\langle\frac{\partial \vec{u}}{\partial t}, N_{t}^{\epsilon}\right\rangle\right)\right|^{2} d \sigma_{t}^{\epsilon} d t .
\end{gathered}
$$

I.e we may consider the latter an equivalent norm.

We will now use a continuity type argument to prove invertibility. We have chosen to present our argument as it is, because of the complexity of the regularity norm, nontrivial.

In the following we will not as before all the time indicate that our quantities depend on $\epsilon$. Let $\alpha \in[0,1]$. Define,

$$
\begin{aligned}
& \rho_{\alpha}(x, t)=\left(\epsilon+\alpha P_{\gamma \epsilon} A(x, t), x, t\right) \\
& \pi_{\alpha}\left(\epsilon+\alpha P_{\gamma \epsilon} A(x, t), x, t\right)=(x, t) \\
& \partial \Omega^{\alpha}=\left\{\left(\epsilon+\alpha P_{\gamma \epsilon} A(x, t), x, t\right): \quad(x, t) \in \mathbb{R}^{n}\right\} .
\end{aligned}
$$

Let the regularity space, $R_{\alpha}\left(\partial \Omega^{\alpha}\right)$, be defined with the equivalent norm

$$
R_{\alpha}\left(\partial \Omega^{\alpha}\right)
$$




$$
=\left\{\vec{u} \in L_{N}^{2}\left(\partial \Omega^{\alpha}\right): \quad\|\vec{u}\|_{L_{1,1 / 2}^{2}\left(\partial \Omega^{\alpha}\right)}+\left(\int_{-\infty}^{\infty} \int_{\partial \Omega_{t}^{\alpha}}\left|S_{t}^{\alpha}\left(\left\langle\frac{\partial \vec{u}}{\partial t}, N_{t}^{\alpha}\right\rangle\right)\right|^{2} d \sigma_{t}^{\alpha} d t\right)^{1 / 2}<\infty\right\} .
$$

In the following we will assume that $S_{\beta}^{-1}$ exists for sufficiently small values of $\beta$. This can be proved using an argument similar to the one presented below and the fact that we have invertibility in the halfplane $\left\{\left(x_{0}, x, t\right): x_{0}>0\right\}$. Recall that if $\vec{u}_{0} \in R_{\alpha}\left(\partial \Omega^{\alpha}\right)$ then by definition $\int_{\partial \Omega_{t}^{\alpha}} \vec{u}_{0} \cdot N_{t}^{\alpha} d \sigma_{t}^{\alpha}=0$ for almost every $t$. All the $\alpha$ 's indicate that things are defined w.r.t $\partial \Omega^{\alpha}$. Given $\vec{u}_{0} \in R_{\alpha}\left(\partial \Omega^{\alpha}\right)$ we define a vector $\vec{v}_{0}$ on $\partial \Omega^{\beta}$ in the following way. For $j \in\{1, \ldots, n-1\}, v_{0}^{j}=\frac{\alpha}{\beta}\left(u_{0}^{j} \circ \rho_{\alpha} \circ \pi_{\beta}\right)$, and $v_{0}^{0}=\left(u_{0} \circ \rho_{\alpha} \circ \pi_{\beta}\right)$. Then for almost every $t, \int_{\partial \Omega_{t}^{\beta}} \vec{v}_{0} \cdot N_{t}^{\beta} d \sigma_{t}^{\beta}=0$.

Suppose that $\vec{u}_{0} \in R_{\alpha}\left(\partial \Omega^{\alpha}\right)$ and suppose that $S_{\beta}^{-1}$ exists. Construct $\vec{v}_{0}$ as above. Then $\vec{v}_{0} \in R_{\beta}\left(\partial \Omega^{\beta}\right)$. Therefore there exists $\vec{g}_{0} \in L^{2}\left(\partial \Omega^{\beta}\right)$ such that $S_{\beta} \vec{g}_{0}=\vec{v}_{0}$. I.e., $\vec{g}_{0}=S_{\beta}^{-1} \vec{v}_{0}$. Define $\vec{f}_{0} \in L^{2}\left(\partial \Omega^{\alpha}\right)$ in the following way,

$$
\overrightarrow{f_{0}}=\left(\vec{g}_{0} \circ \rho_{\beta} \circ \pi_{\alpha}\right) .
$$

Let $A_{\alpha, \beta}$ denote an $n \times n$ matrix having all off diagonal elements equal to zero and $\left(A_{\alpha, \beta}\right)_{11}=1,\left(A_{\alpha, \beta}\right)_{j j}=\frac{\alpha}{\beta}$ for $j \in\{1, \ldots, n-1\}$. Then,

$$
\vec{g}_{0}=S_{\beta}^{-1} \vec{v}_{0}=S_{\beta}^{-1}\left(A_{\alpha, \beta} \vec{u}_{0} \circ \rho_{\alpha} \circ \pi_{\beta}\right) .
$$

I.e.,

$$
A_{\alpha, \beta} \vec{u}_{0}=S_{\beta}\left(\vec{f}_{0} \circ \rho_{\alpha} \circ \pi_{\beta}\right) \circ \rho_{\beta} \circ \pi_{\alpha}
$$

We hope that $S_{\alpha} \vec{f}_{0}$ is a good first approximation of $\vec{u}_{0}$. Define $\vec{u}_{1}=\vec{u}_{0}-S_{\alpha} \vec{f}_{0}$. Repeating the argument above in an iterative fashion we can construct two sequences of vectors $\vec{u}_{j}$ and $\vec{f}_{j}$ in such a way that

$$
\vec{u}_{j+1}=\vec{u}_{j}-S_{\alpha} \vec{f}_{j}, \quad \vec{u}_{0}=\sum_{k=1}^{\infty} S_{\alpha} \vec{f}_{k}
$$

We want to prove that $\sum_{k=0}^{\infty} \vec{f}_{k}$ converges in $L^{2}\left(\partial \Omega^{\alpha}\right)$ if $\alpha-\beta$ is sufficiently small. As above,

$$
\begin{aligned}
\vec{f}_{1} & =\left(\vec{g}_{1} \circ \rho_{\beta} \circ \pi_{\alpha}\right), \\
\vec{g}_{1} & =S_{\beta}^{-1} \vec{v}_{1}=S_{\beta}^{-1}\left(A_{\alpha, \beta} \vec{u}_{1} \circ \rho_{\alpha} \circ \pi_{\beta}\right), \\
A_{\alpha, \beta} \vec{u}_{1} & =A_{\alpha, \beta} \vec{u}_{0}-A_{\alpha, \beta} S_{\alpha} \vec{f}_{0}=S_{\beta}\left(\vec{f}_{0} \circ \rho_{\alpha} \circ \pi_{\beta}\right) \circ \rho_{\beta} \circ \pi_{\alpha}-A_{\alpha, \beta} S_{\alpha} \vec{f}_{0} .
\end{aligned}
$$

Using our Rellich inequality in (2),

$$
\begin{aligned}
& \left\|\vec{f}_{1}\right\|_{L^{2}\left(\partial \Omega^{\alpha}\right)} \sim\left\|\vec{g}_{1}\right\|_{L^{2}\left(\partial \Omega^{\beta}\right)} \sim\left\|A_{\alpha, \beta} \vec{u}_{1} \circ \rho_{\alpha} \circ \pi_{\beta}\right\|_{R_{\beta}\left(\partial \Omega^{\beta}\right)} \\
= & \left\|S_{\beta}\left(\overrightarrow{f_{0}} \circ \rho_{\alpha} \circ \pi_{\beta}\right)-A_{\alpha, \beta} S_{\alpha} \vec{f}_{0} \circ \rho_{\alpha} \circ \pi_{\beta}\right\|_{R_{\beta}\left(\partial \Omega^{\beta}\right)} .
\end{aligned}
$$

Define, $T_{\alpha, \beta} \overrightarrow{f_{0}}=S_{\beta}\left(\overrightarrow{f_{0}} \circ \rho_{\alpha} \circ \pi_{\beta}\right)-A_{\alpha, \beta} S_{\alpha} \overrightarrow{f_{0}} \circ \rho_{\alpha} \circ \pi_{\beta}$. 
We want to estimate the operator norm of this operator in $R_{\beta}\left(\partial \Omega^{\beta}\right)$. For simplicity we surpress the index of $\vec{f}_{0}$. We consider $T_{\alpha, \beta} \vec{f}$. Then

$$
\left\|T_{\alpha, \beta} \vec{f}\right\|_{L_{1,1 / 2}^{2}\left(\partial \Omega^{\beta}\right)}^{2}=\left\|\mathbb{D}\left(T_{\alpha, \beta} \vec{f} \circ \rho_{\beta}\right)\right\|_{L^{2}\left(\mathbb{R}^{n}\right)}^{2}
$$

and

$$
\begin{aligned}
T_{\alpha, \beta} \vec{f} \circ \rho_{\beta} & =S_{\beta}\left(\vec{f} \circ \rho_{\alpha} \circ \pi_{\beta}\right) \circ \rho_{\beta}-A_{\alpha, \beta} S_{\alpha} \vec{f} \circ \rho_{\alpha} \\
& =\int_{-\infty}^{\infty} \int_{\mathbb{R}^{n-1}} \Gamma\left(\rho_{\beta}(x, t)-\rho_{\beta}(y, s)\right) \vec{f} \circ \rho_{\alpha}(y, s) \sqrt{1+\beta^{2}\left|\nabla P_{\gamma \epsilon} A(y, s)\right|^{2}} d y d s \\
& -\int_{-\infty}^{\infty} \int_{\mathbb{R}^{n-1}} \Gamma\left(\rho_{\alpha}(x, t)-\rho_{\alpha}(y, s)\right) \vec{f} \circ \rho_{\alpha}(y, s) \sqrt{1+\alpha^{2}\left|\nabla P_{\gamma \epsilon} A(y, s)\right|^{2}} d y d s .
\end{aligned}
$$

Define a kernel in the following way,

$$
\begin{aligned}
& K_{\alpha, \beta}(x, t, y, s) \\
:= & \Gamma\left(\rho_{\beta}(x, t)-\rho_{\beta}(y, s)\right) \sqrt{1+\beta^{2}\left|\nabla P_{\gamma \epsilon} A(y, s)\right|^{2}} \\
- & \Gamma\left(\rho_{\alpha}(x, t)-\rho_{\alpha}(y, s)\right) \sqrt{1+\alpha^{2}\left|\nabla P_{\gamma \epsilon} A(y, s)\right|^{2}} \\
= & {\left[\Gamma\left(\rho_{\beta}(x, t)-\rho_{\beta}(y, s)\right)-\Gamma\left(\rho_{\alpha}(x, t)-\rho_{\alpha}(y, s)\right)\right] \times \sqrt{1+\beta^{2}\left|\nabla P_{\gamma \epsilon} A(y, s)\right|^{2}} } \\
+ & \Gamma\left(\rho_{\alpha}(x, t)-\rho_{\alpha}(y, s)\right)\left[\sqrt{1+\beta^{2}\left|\nabla P_{\gamma \epsilon} A(y, s)\right|^{2}}-\sqrt{1+\alpha^{2}\left|\nabla P_{\gamma \epsilon} A(y, s)\right|^{2}}\right] .
\end{aligned}
$$

Obviously,

$$
\begin{gathered}
{\left[\sqrt{1+\beta^{2}\left|\nabla P_{\gamma \epsilon} A(y, s)\right|^{2}}-\sqrt{1+\alpha^{2}\left|\nabla P_{\gamma \epsilon} A(y, s)\right|^{2}}\right]=} \\
{\left[\sqrt{1+\beta^{2}\left|\nabla P_{\gamma \epsilon} A(y, s)\right|^{2}}+\sqrt{1+\alpha^{2}\left|\nabla P_{\gamma \epsilon} A(y, s)\right|^{2}}\right]}
\end{gathered}
$$

Define,

$$
\begin{aligned}
K_{\alpha, \beta}^{1}(x, t, y, s) & =\left[\Gamma\left(\rho_{\beta}(x, t)-\rho_{\beta}(y, s)\right)-\Gamma\left(\rho_{\alpha}(x, t)-\rho_{\alpha}(y, s)\right)\right] \\
K_{\alpha, \beta}^{2}(x, t, y, s) & =\Gamma\left(\rho_{\alpha}(x, t)-\rho_{\alpha}(y, s)\right), \\
\vec{F}(y, s) & =\vec{f} \circ \rho_{\alpha}(y, s) \sqrt{1+\beta^{2}\left|\nabla P_{\gamma \epsilon} A(y, s)\right|^{2}} \\
\vec{G}(y, s) & =\vec{f} \circ \rho_{\alpha}(y, s) \frac{(\alpha+\beta)\left|\nabla P_{\gamma \epsilon} A(y, s)\right|^{2}}{\left[\sqrt{1+\beta^{2}\left|\nabla P_{\gamma \epsilon} A(y, s)\right|^{2}}+\sqrt{1+\alpha^{2}\left|\nabla P_{\gamma \epsilon} A(y, s)\right|^{2}}\right]} .
\end{aligned}
$$

Let $T_{\alpha, \beta}^{i}$ be the operator corresponding to the kernel $K_{\alpha, \beta}^{i}(x, t, y, s)$. Then,

$$
T_{\alpha, \beta} \vec{f} \circ \rho_{\beta}(x, t)=T_{\alpha, \beta}^{1} \vec{F}(x, t)-(\alpha-\beta) T_{\alpha, \beta}^{2} \vec{G}(x, t) .
$$


Using the results of Hofmann[18] we immediately get,

$$
\left\|\mathbb{D}\left(T_{\alpha, \beta}^{2} \vec{G}\right)\right\|_{L^{2}\left(\mathbb{R}^{n}\right)}^{2} \lesssim\|\vec{f}\|_{L^{2}\left(\partial \Omega^{\alpha}\right)} .
$$

The fundamental theorem of calculus gives,

$$
\begin{aligned}
& K_{\alpha, \beta}^{1}(x, t, y, s) \\
= & -\int_{\beta}^{\alpha} \frac{\partial}{\partial \sigma} \Gamma\left(\sigma P_{\gamma \epsilon} A(x, t)-\sigma P_{\gamma \epsilon} A(y, s), x-y, t-s\right) d \sigma \\
= & -\int_{\beta}^{\alpha} \Gamma_{x_{0}}\left(\sigma\left(P_{\gamma \epsilon} A(x, t)-P_{\gamma \epsilon} A(y, s)\right), x-y, t-s\right)\left(P_{\gamma \epsilon} A(x, t)-P_{\gamma \epsilon} A(y, s)\right) d \sigma .
\end{aligned}
$$

Again the continuity of the operator $T_{\alpha, \beta}^{1}$ follows from the result of Hofmann[18] and we may conclude that,

$$
\left\|T_{\alpha, \beta} \vec{f}\right\|_{L_{1,1 / 2}^{2}\left(\partial \Omega^{\beta}\right)}^{2} \leq C(\alpha-\beta)\|\vec{f}\|_{L^{2}\left(\partial \Omega^{\alpha}\right)}
$$

where $C$ is a constant independent of $\alpha$ and $\beta$.

We now have to consider the rest of the norm, i.e.,

$$
\int_{-\infty}^{\infty} \int_{\partial \Omega_{t}^{\beta}}\left|S_{t}^{\beta}\left(\left\langle\frac{\partial T_{\alpha, \beta} \vec{f}}{\partial t}, N_{t}^{\beta}\right\rangle\right)\right|^{2} d \sigma_{t}^{\beta} d t
$$

Recall that

$$
T_{\alpha, \beta} \vec{f} \circ \rho_{\beta}=S_{\beta}\left(\vec{f} \circ \rho_{\alpha} \circ \pi_{\beta}\right) \circ \rho_{\beta}-A_{\alpha, \beta} S_{\alpha} \vec{f} \circ \rho_{\alpha} .
$$

Let $p^{\beta}$ and $p^{\alpha}$ be the pressures associated to $S_{\beta}\left(\vec{f} \circ \rho_{\alpha} \circ \pi_{\beta}\right)$ and $S_{\alpha} \vec{f}$ respectively. By definition if $(P, t) \in \partial \Omega^{\beta}$

$$
\begin{aligned}
S_{t}^{\beta}\left(\left\langle\frac{\partial T_{\alpha, \beta} \vec{f}}{\partial t}, N_{t}^{\beta}\right\rangle\right)(P, t) & =p \cdot v \int_{\partial \Omega_{t}^{\beta}} \frac{\left\langle\frac{\partial T_{\alpha, \beta} \vec{f}}{\partial t}, N_{t}^{\beta}\right\rangle(Q, t)}{w_{n}(2-n)|P-Q|^{n-2}} d \sigma_{t}^{\beta}(Q) \\
& =p \cdot v \int_{\partial \Omega_{t}^{\beta}} \frac{\left\langle\frac{\partial}{\partial t} S_{\beta}\left(\vec{f} \circ \rho_{\alpha} \circ \pi_{\beta}\right), N_{t}^{\beta}\right\rangle(Q, t)}{w_{n}(2-n)|P-Q|^{n-2}} d \sigma_{t}^{\beta}(Q) \\
& -p \cdot v \int_{\partial \Omega_{t}^{\beta}} \frac{\left\langle\frac{\partial}{\partial t}\left(A_{\alpha, \beta} S_{\alpha} \vec{f} \circ \rho_{\alpha} \circ \pi_{\beta}\right), N_{t}^{\beta}\right\rangle(Q, t)}{w_{n}(2-n)|P-Q|^{n-2}} d \sigma_{t}^{\beta}(Q) \\
& :=I_{1}(P, t)+I_{2}(P, t) .
\end{aligned}
$$

We now want to spell out these expression in graph coordinates on $\partial \Omega^{\beta}$. Let for the moment $\vec{v}=S_{\beta}\left(\vec{f} \circ \rho_{\alpha} \circ \pi_{\beta}\right)$ and consider $S_{t}^{\beta}\left(\left\langle\Delta \vec{v}, N_{t}^{\beta}\right\rangle(P, t) . \quad\left\langle\Delta \vec{v}, N_{t}^{\beta}\right\rangle=\right.$ $\sum_{i=0}^{n-1} \Delta v^{i} N_{t}^{\beta, i}$. Here,

$$
N_{t}^{\beta, i}=\beta \frac{\partial P_{\gamma \epsilon} A}{\partial x_{i}} \cdot \frac{1}{\left|N_{t}^{\beta}\right|} \text { for } i \in\{1, \ldots, n-1\}, \quad N_{t}^{\epsilon, 0}=-\frac{1}{\left|N_{t}^{\beta}\right|} .
$$


Note that on $\partial \Omega_{t}^{\beta}$ we have $x_{0}=\epsilon+\beta P_{\gamma \epsilon} A(x, t)$. Let

$$
T_{j}^{\beta}:=\left(\beta \frac{\partial P_{\gamma \epsilon} A}{\partial x_{j}}, 0,,,,, 0,1,0, \ldots 0\right) /\left|N_{t}^{\beta}\right|,
$$

where we have placed the number 1 at position $j$. Here $j \in\{1, \ldots, n-1\}$. Note that $T_{j}$ is tangent to $\partial \Omega_{t}^{\beta}$. Using the formula of Shen stated in Lemma 7.4 and partial integration,

$$
\begin{aligned}
& S_{t}^{\beta}\left(\left\langle\Delta \vec{v}, N_{t}^{\beta}\right\rangle(P, t)\right. \\
& =p \cdot v \int_{\partial \Omega_{t}^{\beta}} \frac{\left\langle\left(\Delta \vec{v}, N_{t}^{\beta}\right\rangle\right)(Q, t)}{w_{n}(2-n)|P-Q|^{n-2}} d \sigma_{t}^{\beta}(Q) \\
& =\sum_{j, k=1}^{n-1} \int_{\partial \Omega_{t}^{\beta}} \frac{\beta}{w_{n}(2-n)|P-Q|^{n-2}}\left[\frac{\partial}{\partial T_{j}^{\beta}}\left(\frac{\partial v^{k}}{\partial x_{j}} \frac{\partial P_{\gamma \epsilon} A}{\partial x_{k}}\right)-\frac{\partial}{\partial T_{k}^{\beta}}\left(\frac{\partial v^{k}}{\partial x_{j}} \frac{\partial P_{\gamma \epsilon} A}{\partial x_{j}}\right)\right] d \sigma_{t}^{\beta} \\
& -\sum_{j=1}^{n-1} \int_{\partial \Omega_{t}^{\beta}} \frac{1}{w_{n}(2-n)|P-Q|^{n-2}} \frac{\partial}{\partial T_{j}^{\beta}}\left(\frac{\partial v^{0}}{\partial x_{j}}\right) d \sigma_{t}^{\beta} \\
& +\sum_{k=1}^{n-1} \int_{\partial \Omega_{t}^{\beta}} \frac{1}{w_{n}(2-n)|P-Q|^{n-2}} \frac{\partial}{\partial T_{k}^{\beta}}\left(\frac{\partial v^{k}}{\partial x_{0}}\right) d \sigma_{t}^{\beta} \\
& =-\sum_{j, k=1}^{n-1} \int_{\partial \Omega_{t}^{\beta}} \frac{\partial}{\partial T_{j}^{\beta}}\left(\frac{\beta}{w_{n}(2-n)|P-Q|^{n-2}}\right)\left(\frac{\partial v^{k}}{\partial x_{j}} \frac{\partial P_{\gamma \epsilon} A}{\partial x_{k}}\right) d \sigma_{t}^{\beta} \\
& +\sum_{j, k=1}^{n-1} \int_{\partial \Omega_{t}^{\beta}} \frac{\partial}{\partial T_{k}^{\beta}}\left(\frac{\beta}{w_{n}(2-n)|P-Q|^{n-2}}\right)\left(\frac{\partial v^{k}}{\partial x_{j}} \frac{\partial P_{\gamma \epsilon} A}{\partial x_{j}}\right) d \sigma_{t}^{\beta} \\
& +\sum_{j=1}^{n-1} \int_{\partial \Omega_{t}^{\beta}} \frac{\partial}{\partial T_{j}^{\beta}}\left(\frac{1}{w_{n}(2-n)|P-Q|^{n-2}}\right)\left(\frac{\partial v^{0}}{\partial x_{j}}\right) d \sigma_{t}^{\beta} \\
& -\sum_{k=1}^{n-1} \int_{\partial \Omega_{t}^{\beta}} \frac{\partial}{\partial T_{k}^{\beta}}\left(\frac{1}{w_{n}(2-n)|P-Q|^{n-2}}\right)\left(\frac{\partial v^{k}}{\partial x_{0}}\right) d \sigma_{t}^{\beta} .
\end{aligned}
$$

Changing to graph coordinates and introducing,

$$
K(\beta, x, y, t)=\left(\beta\left(P_{\gamma \epsilon} A(x, t)-P_{\gamma \epsilon} A(y, t)\right), x-y\right)
$$

$$
\begin{aligned}
& S_{t}^{\beta}\left(\left\langle\Delta \vec{v}, N_{t}^{\beta}\right\rangle \circ \rho_{\beta}(y, t)=\right. \\
- & \sum_{j, k=1}^{n-1} \int_{\mathbb{R}^{n-1}} \beta\left[\beta^{2} \frac{\partial P_{\gamma \epsilon} A}{\partial x_{j}} \frac{P_{\gamma \epsilon} A(y, t)-P_{\gamma \epsilon} A(z, t)}{w_{n}|K(\beta, y, z, t)|^{n}}+\frac{y_{j}-z_{j}}{w_{n}|K(\beta, y, z, t)|^{n}}\right] \frac{\partial P_{\gamma \epsilon} A}{\partial x_{k}} \frac{\partial v^{k}}{\partial x_{j}} \circ \rho_{\beta}(z, t) d z
\end{aligned}
$$




$$
\begin{aligned}
& +\sum_{j, k=1_{\mathbb{R}^{n-1}}}^{n-1} \beta\left[\beta^{2} \frac{\partial P_{\gamma \epsilon} A}{\partial x_{k}} \frac{P_{\gamma \epsilon} A(y, t)-P_{\gamma \epsilon} A(z, t)}{w_{n}|K(\beta, y, z, t)|^{n}}+\frac{y_{k}-z_{k}}{w_{n}|K(\beta, y, z, t)|^{n}}\right] \frac{\partial P_{\gamma \epsilon} A}{\partial x_{j}} \frac{\partial v^{k}}{\partial x_{j}} \circ \rho_{\beta}(z, t) d z \\
& +\sum_{j=1}^{n-1} \int_{\mathbb{R}^{n-1}}\left[\beta^{2} \frac{\partial P_{\gamma \epsilon} A}{\partial x_{j}} \frac{P_{\gamma \epsilon} A(y, t)-P_{\gamma \epsilon} A(z, t)}{w_{n}|K(\beta, y, z, t)|^{n}}+\frac{y_{j}-z_{j}}{w_{n}|K(\beta, y, z, t)|^{n}}\right]\left(\frac{\partial v^{0}}{\partial x_{j}}\right) \circ \rho_{\beta}(z, t) d z \\
& -\sum_{k=1}^{n-1} \int_{\mathbb{R}^{n-1}}\left[\beta^{2} \frac{\partial P_{\gamma \epsilon} A}{\partial x_{k}} \frac{P_{\gamma \epsilon} A(y, t)-P_{\gamma \epsilon} A(z, t)}{w_{n}|K(\beta, y, z, t)|^{n}}+\frac{y_{k}-z_{k}}{w_{n}|K(\beta, y, z, t)|^{n}}\right]\left(\frac{\partial v^{k}}{\partial x_{0}}\right) \circ \rho_{\beta}(z, t) d z .
\end{aligned}
$$

We also recall that $S_{t}^{\beta}\left(\frac{\partial p^{\beta}}{\partial N_{t}^{\beta}}\right)(P, t)=\left(\frac{I}{2}-K_{t}^{\beta}\right)\left(p^{\beta}\right)(P, t)$ where

$$
K_{t}^{\beta}\left(p^{\beta}\right)(P, t):=p . v \int_{\partial \Omega_{t}^{\beta}} \frac{\left\langle P-Q, N_{t}^{\beta}(Q)\right\rangle(Q, t)}{w_{n}|P-Q|^{n}} p^{\beta}(Q, t) d \sigma_{t}^{\beta}(Q) .
$$

Using the formulas just developed we will write down a formula for $I_{1} \circ \rho_{\beta}(y, t)$ below. But first we focus on $I_{2}$. If $\left(p_{0}, p, t\right) \in \partial \Omega_{t}^{\beta}$ then $\pi_{\beta}\left(p_{0}, p, t\right)=(p, t)$. Consider

$$
-I_{2} \circ \rho_{\beta}(x, t)=\int_{\mathbb{R}^{n-1}} \frac{\left\langle\frac{\partial}{\partial t}\left(A_{\alpha, \beta} S_{\alpha} \vec{f} \circ \rho_{\alpha} \circ \pi_{\beta}\right) \circ \rho_{\beta}, N_{t}^{\beta}\right\rangle}{w_{n}(2-n)\left|\rho_{\beta}(x, t)-\rho_{\beta}(y, t)\right|^{n-2}} \sqrt{1+\beta^{2}\left|\nabla P_{\gamma \epsilon} A(y, t)\right|^{2}} d y .
$$

Let $H:=A_{\alpha, \beta} S_{\alpha} \vec{f} \circ \rho_{\alpha} \circ \pi_{\beta}$ be defined on $\partial \Omega^{\beta}$. Then $H \circ \rho_{\beta}=A_{\alpha, \beta} S_{\alpha} \vec{f} \circ \rho_{\alpha}$ and,

$$
\frac{\partial}{\partial t}\left(H \circ \rho_{\beta}\right)=H_{t} \circ \rho_{\beta}+\beta H_{x_{0}} \circ \rho_{\beta} \frac{\partial P_{\gamma \epsilon} A}{\partial t} .
$$

I.e.,

$$
\begin{aligned}
\frac{\partial}{\partial t}\left(A_{\alpha, \beta} S_{\alpha} \vec{f} \circ \rho_{\alpha} \circ \pi_{\beta}\right) \circ \rho_{\beta} & =\frac{\partial}{\partial t}\left(A_{\alpha, \beta} S_{\alpha} \vec{f} \circ \rho_{\alpha}\right) \\
& -\beta \frac{\partial}{\partial x_{0}}\left(A_{\alpha, \beta} S_{\alpha} \vec{f} \circ \rho_{\alpha} \circ \pi_{\beta}\right) \circ \rho_{\beta} \frac{\partial P_{\gamma \epsilon} A}{\partial t} \\
& =A_{\alpha, \beta} \frac{\partial S_{\alpha} \vec{f}}{\partial t} \circ \rho_{\alpha}+\alpha A_{\alpha, \beta}\left(\frac{\partial S_{\alpha} \vec{f}}{\partial x_{0}} \circ \rho_{\alpha}\right) \frac{\partial P_{\gamma \epsilon} A}{\partial t} \\
& -\frac{\beta \frac{\partial P_{\gamma \epsilon} A}{\partial t}}{1+\beta \frac{\partial P_{\gamma \epsilon} A}{\partial \epsilon}} \frac{\partial}{\partial \epsilon}\left(A_{\alpha, \beta} S_{\alpha} \vec{f} \circ \rho_{\alpha}\right) .
\end{aligned}
$$

But

$$
\frac{\partial}{\partial \epsilon}\left(A_{\alpha, \beta} S_{\alpha} \vec{f} \circ \rho_{\alpha}\right)=\left(A_{\alpha, \beta} \frac{\partial S_{\alpha} \vec{f}}{\partial x_{0}} \circ \rho_{\alpha}\right)\left(1+\alpha \frac{\partial P_{\gamma \epsilon} A}{\partial \epsilon}\right) .
$$

We may therefore conclude that,

$$
\begin{aligned}
\frac{\partial}{\partial t}\left(A_{\alpha, \beta} S_{\alpha} \vec{f} \circ \rho_{\alpha} \circ \pi_{\beta}\right) \circ \rho_{\beta} & =A_{\alpha, \beta} \frac{\partial S_{\alpha} \vec{f}}{\partial t} \circ \rho_{\alpha}+\alpha A_{\alpha, \beta}\left(\frac{\partial S_{\alpha} \vec{f}}{\partial x_{0}} \circ \rho_{\alpha}\right) \frac{\partial P_{\gamma \epsilon} A}{\partial t} \\
& -\beta \frac{\partial P_{\gamma \epsilon} A}{\partial t} \frac{\left(1+\alpha \frac{\partial P_{\gamma \epsilon} A}{\partial \epsilon}\right)}{\left(1+\beta \frac{\partial P_{\gamma \epsilon} A}{\partial \epsilon}\right)}\left(A_{\alpha, \beta} \frac{\partial S_{\alpha} \vec{f}}{\partial x_{0}} \circ \rho_{\alpha}\right) .
\end{aligned}
$$


Using these calculations we see that the expression,

$$
\int_{\mathbb{R}^{n-1}} \frac{\left\langle\left(\frac{\partial}{\partial t}\left(A_{\alpha, \beta} S_{\alpha} \vec{f} \circ \rho_{\alpha} \circ \pi_{\beta}\right) \circ \rho_{\beta}, N_{t}^{\beta}\right\rangle\right)}{w_{n}(2-n)\left|\rho_{\beta}(x, t)-\rho_{\beta}(y, t)\right|^{n-2}} \sqrt{1+\beta^{2}\left|\nabla P_{\gamma \epsilon} A(y, t)\right|^{2}} d y
$$

equals

$$
\begin{aligned}
& \int_{\mathbb{R}^{n-1}} \frac{\left\langle A_{\alpha, \beta} \frac{\partial S_{\alpha} \vec{f}}{\partial t} \circ \rho_{\alpha}, N_{t}^{\beta}\right\rangle}{w_{n}(2-n)\left|\rho_{\beta}(x, t)-\rho_{\beta}(y, t)\right|^{n-2}} \sqrt{1+\beta^{2}\left|\nabla P_{\gamma \epsilon} A(y, t)\right|^{2}} d y \\
& +\int_{\mathbb{R}^{n-1}} \frac{\left(\alpha-\beta \frac{\left(1+\alpha \frac{\partial P_{\gamma \epsilon} A}{\partial \epsilon}\right)}{\left(1+\beta \frac{\partial P_{\gamma \epsilon} A}{\partial \epsilon}\right)}\right)\left\langle A_{\alpha, \beta} \frac{\partial S_{\alpha} \vec{f}}{\partial x_{0}} \circ \rho_{\alpha}, N_{t}^{\beta}\right\rangle}{w_{n}(2-n)\left|\rho_{\beta}(x, t)-\rho_{\beta}(y, t)\right|^{n-2}} \frac{\partial P_{\gamma \epsilon} A}{\partial t} \sqrt{1+\beta^{2}\left|\nabla P_{\gamma \epsilon} A(y, t)\right|^{2}} d y \\
& :=\left(I_{21}+I_{22}\right) \circ \rho_{\beta}(x, t)
\end{aligned}
$$

Note that,

$$
\left(\alpha-\beta \frac{1+\alpha \frac{\partial P_{\gamma \epsilon} A}{\partial \epsilon}}{1+\beta \frac{\partial P_{\gamma \epsilon} A}{\partial \epsilon}}\right)=\frac{\alpha-\beta}{1+\beta \frac{\partial P_{\gamma \epsilon} A}{\partial \epsilon}} .
$$

Therefore,

$$
\int_{-\infty}^{\infty} \int_{\mathbb{R}^{n-1}}\left(I_{22} \circ \rho_{\beta}(x, t)\right)^{2} d x d t \leq C(\alpha-\beta)\left\|\nabla S_{\alpha} \vec{f}\right\|_{L^{2}\left(\partial \Omega^{\alpha}\right)}^{2} \leq C(\alpha-\beta)\|\vec{f}\|_{L^{2}\left(\partial \Omega^{\alpha}\right)}^{2} .
$$

We now focus on $I_{21}$. Retreating to ordinary coordinates and again using the formula of Shen we have

$$
\begin{aligned}
& I_{21}\left(p_{0}, p, t\right) \\
= & p \cdot v \int_{\partial \Omega_{t}^{\alpha}} \frac{\left\langle\frac{\partial S_{\alpha} \vec{f}}{\partial t}, N_{t}^{\alpha}\right\rangle}{w_{n}(2-n)\left|\left(\left(p_{0}-\frac{\beta}{\alpha} q_{0}+\left(\frac{\beta}{\alpha}-1\right) \epsilon\right), p-q\right)\right|^{n-2}} d \sigma_{t}^{\alpha}(Q) \\
= & \sum_{j, k=1}^{n-1} \int_{\partial \Omega_{t}^{\alpha}} \frac{\alpha}{w_{n}(2-n)\left|\left(\left(p_{0}-\frac{\beta}{\alpha} q_{0}+\left(\frac{\beta}{\alpha}-1\right) \epsilon\right), p-q\right)\right|^{n-2}} \frac{\partial}{\partial T_{j}^{\alpha}}\left(\frac{\partial\left(S_{\alpha} \vec{f}\right)^{k}}{\partial x_{j}} \frac{\partial P_{\gamma \epsilon} A}{\partial x_{k}}\right) d \sigma_{t}^{\alpha} \\
- & \sum_{j, k=1}^{n-1} \int_{\partial \Omega_{t}^{\alpha}} \frac{\alpha}{w_{n}(2-n)\left|\left(\left(p_{0}-\frac{\beta}{\alpha} q_{0}+\left(\frac{\beta}{\alpha}-1\right) \epsilon\right), p-q\right)\right|^{n-2}} \frac{\partial}{\partial T_{k}^{\alpha}}\left(\frac{\partial\left(S_{\alpha} \vec{f}\right)^{k}}{\partial x_{j}} \frac{\partial P_{\gamma \epsilon} A}{\partial x_{j}}\right) d \sigma_{t}^{\alpha} \\
- & \sum_{j=1}^{n-1} \int_{\partial \Omega_{t}^{\alpha}} \frac{1}{w_{n}(2-n)\left|\left(\left(p_{0}-\frac{\beta}{\alpha} q_{0}+\left(\frac{\beta}{\alpha}-1\right) \epsilon\right), p-q\right)\right|^{n-2}} \frac{\partial}{\partial T_{j}^{\alpha}}\left(\frac{\partial\left(S_{\alpha} \vec{f}\right)^{0}}{\partial x_{j}}\right) d \sigma_{t}^{\alpha} \\
+ & \left.\sum_{k=1}^{n-1} \int_{\partial \Omega_{t}^{\alpha}} \frac{\partial}{w_{n}(2-n)\left|\left(\left(p_{0}-\frac{\beta}{\alpha} q_{0}+\left(\frac{\beta}{\alpha}-1\right) \epsilon\right), p-q\right)\right|^{n-2}} \frac{\partial\left(S_{\alpha} \vec{f}\right)^{k}}{\partial T_{k}^{\alpha}}\right) d \sigma_{t}^{\alpha} \\
- & p \cdot v \int_{\partial \Omega_{t}^{\alpha}} \frac{\left\langle\nabla p^{\alpha}, N_{t}^{\alpha}\right\rangle}{w_{n}(2-n)\left|\left(\left(p_{0}-\frac{\beta}{\alpha} q_{0}+\left(\frac{\beta}{\alpha}-1\right) \epsilon\right), p-q\right)\right|^{n-2}} d \sigma_{t}^{\alpha}(Q)
\end{aligned}
$$




$$
\begin{aligned}
& =-\sum_{j, k=1}^{n-1} \int_{\partial \Omega_{t}^{\alpha}} \frac{\partial}{\partial T_{j}^{\alpha}}\left(\frac{\alpha}{\left.w_{n}(2-n) \mid\left(\left(p_{0}-\frac{\beta}{\alpha} q_{0}+\left(\frac{\beta}{\alpha}-1\right) \epsilon\right), p-q\right)\right)\left.\right|^{n-2}}\right)\left(\frac{\partial\left(S_{\alpha} \vec{f}\right)^{k}}{\partial x_{j}} \frac{\partial P_{\gamma \epsilon} A}{\partial x_{k}}\right) d \sigma_{t}^{\alpha} \\
& +\sum_{j, k=1}^{n-1} \int_{\partial \Omega_{t}^{\alpha}} \frac{\partial}{\partial T_{k}^{\alpha}}\left(\frac{\alpha}{\left.w_{n}(2-n) \mid\left(\left(p_{0}-\frac{\beta}{\alpha} q_{0}+\left(\frac{\beta}{\alpha}-1\right) \epsilon\right), p-q\right)\right)\left.\right|^{n-2}}\right)\left(\frac{\partial\left(S_{\alpha} \vec{f}\right)^{k}}{\partial x_{j}} \frac{\partial P_{\gamma \epsilon} A}{\partial x_{j}}\right) d \sigma_{t}^{\alpha} \\
& +\sum_{j=1}^{n-1} \int_{\partial \Omega_{t}^{\alpha}} \frac{\partial}{\partial T_{j}^{\alpha}}\left(\frac{1}{\left.w_{n}(2-n) \mid\left(\left(p_{0}-\frac{\beta}{\alpha} q_{0}+\left(\frac{\beta}{\alpha}-1\right) \epsilon\right), p-q\right)\right)\left.\right|^{n-2}}\right)\left(\frac{\partial\left(S_{\alpha} \vec{f}\right)^{0}}{\partial x_{j}}\right) d \sigma_{t}^{\alpha} \\
& -\sum_{k=1}^{n-1} \int_{\partial \Omega_{t}^{\alpha}} \frac{\partial}{\partial T_{k}^{\alpha}}\left(\frac{1}{\left.w_{n}(2-n) \mid\left(\left(p_{0}-\frac{\beta}{\alpha} q_{0}+\left(\frac{\beta}{\alpha}-1\right) \epsilon\right), p-q\right)\right)\left.\right|^{n-2}}\right)\left(\frac{\partial\left(S_{\alpha} \vec{f}\right)^{k}}{\partial x_{0}}\right) d \sigma_{t}^{\alpha} \\
& -p . v \int_{\partial \Omega_{t}^{\alpha}} \frac{\left\langle\nabla p^{\alpha}, N_{t}^{\alpha}\right\rangle}{w_{n}(2-n)\left|\left(\left(p_{0}-\frac{\beta}{\alpha} q_{0}+\left(\frac{\beta}{\alpha}-1\right) \epsilon\right), p-q\right)\right|^{n-2}} d \sigma_{t}^{\alpha}(Q) .
\end{aligned}
$$

By arguments similar to those used in the proof of Lemma 7.2 we have

$$
\begin{gathered}
\int_{\partial \Omega_{t}^{\alpha}} \frac{\left\langle\nabla p^{\alpha}, N_{t}^{\alpha}\right\rangle}{w_{n}(2-n)\left|\left(\left(p_{0}-\frac{\beta}{\alpha} q_{0}+\left(\frac{\beta}{\alpha}-1\right) \epsilon\right), p-q\right)\right|^{n-2}} d \sigma_{t}^{\alpha}(Q)= \\
\frac{1}{2} p^{\alpha}(P, t)-p \cdot v \int_{\partial \Omega_{t}^{\alpha}} \frac{\left\langle\left(\left(p_{0}-\frac{\beta}{\alpha} q_{0}+\left(\frac{\beta}{\alpha}-1\right) \epsilon\right), p-q\right), N_{t}^{\beta}(Q)\right\rangle}{w_{n}\left|\left(\left(p_{0}-\frac{\beta}{\alpha} q_{0}+\left(\frac{\beta}{\alpha}-1\right) \epsilon\right), p-q\right)\right|^{n}} p^{\alpha}(Q, t) d \sigma_{t}^{\beta}(Q) .
\end{gathered}
$$

Changing to graph coordinates

$$
\begin{aligned}
& I_{21} \circ \rho_{\beta}(y, t)= \\
& -\sum_{j, k=1}^{n-1} \int_{\mathbb{R}^{n-1}} \alpha\left[\beta^{2} \frac{\partial P_{\gamma \epsilon} A}{\partial x_{j}} \frac{P_{\gamma \epsilon} A(y, t)-P_{\gamma \epsilon} A(z, t)}{w_{n}|K(\beta, y, z, t)|^{n}}+\frac{y_{j}-z_{j}}{w_{n}|K(\beta, y, z, t)|^{n}}\right] \\
& \times \frac{\partial P_{\gamma \epsilon} A}{\partial x_{k}} \frac{\partial\left(S_{\alpha} \vec{f}\right)^{k}}{\partial x_{j}} \circ \rho_{\alpha}(z, t) d z \\
& +\sum_{j, k=1}^{n-1} \int_{\mathbb{R}^{n-1}} \alpha\left[\beta^{2} \frac{\partial P_{\gamma \epsilon} A}{\partial x_{k}} \frac{P_{\gamma \epsilon} A(y, t)-P_{\gamma \epsilon} A(z, t)}{w_{n}|K(\beta, y, z, t)|^{n}}+\frac{y_{k}-z_{k}}{w_{n}|K(\beta, y, z, t)|^{n}}\right] \\
& \times \frac{\partial P_{\gamma \epsilon} A}{\partial x_{j}} \frac{\partial\left(S_{\alpha} \vec{f}\right)^{k}}{\partial x_{j}} \circ \rho_{\alpha}(z, t) d z \\
& +\sum_{j=1}^{n-1} \int_{\mathbb{R}^{n-1}}\left[\beta^{2} \frac{\partial P_{\gamma \epsilon} A}{\partial x_{j}} \frac{P_{\gamma \epsilon} A(y, t)-P_{\gamma \epsilon} A(z, t)}{w_{n}|K(\beta, y, z, t)|^{n}}+\frac{y_{j}-z_{j}}{w_{n}|K(\beta, y, z, t)|^{n}}\right] \\
& \times\left(\frac{\partial\left(S_{\alpha} \vec{f}\right)^{0}}{\partial x_{j}}\right) \circ \rho_{\alpha}(z, t) d z \\
& -\sum_{k=1}^{n-1} \int_{\mathbb{R}^{n-1}}\left[\beta^{2} \frac{\partial P_{\gamma \epsilon} A}{\partial x_{k}} \frac{P_{\gamma \epsilon} A(y, t)-P_{\gamma \epsilon} A(z, t)}{w_{n}|K(\beta, y, z, t)|^{n}}+\frac{y_{k}-z_{k}}{w_{n}|K(\beta, y, z, t)|^{n}}\right]
\end{aligned}
$$




$$
\begin{gathered}
\times\left(\frac{\partial\left(S_{\alpha} \vec{f}\right)^{k}}{\partial x_{0}}\right) \circ \rho_{\alpha}(z, t) d z \\
-\frac{1}{2} p^{\alpha}(P, t)+p . v \int_{\partial \Omega_{t}^{\alpha}} \frac{\left\langle\left(\left(p_{0}-\frac{\beta}{\alpha} q_{0}+\left(\frac{\beta}{\alpha}-1\right) \epsilon\right), p-q\right), N_{t}^{\beta}(Q)\right\rangle}{w_{n}\left|\left(\left(p_{0}-\frac{\beta}{\alpha} q_{0}+\left(\frac{\beta}{\alpha}-1\right) \epsilon\right), p-q\right)\right|^{n}} p^{\alpha}(Q, t) d \sigma_{t}^{\beta}(Q) .
\end{gathered}
$$

Before continuing we have to summarize what we have achieved so far. Recall that

$$
T_{\alpha, \beta} \vec{f}=S_{\beta}\left(\vec{f} \circ \rho_{\alpha} \circ \pi_{\beta}\right)-A_{\alpha, \beta} S_{\alpha} \vec{f} \circ \rho_{\alpha} \circ \pi_{\beta}
$$

and that our main objective is to prove the estimate

$$
\left\|T_{\alpha, \beta} \vec{f}\right\|_{R_{\beta}\left(\partial \Omega^{\beta}\right)} \leq C(\alpha-\beta)\|\vec{f}\|_{L^{2}\left(\partial \Omega^{\alpha}\right)} .
$$

But

$$
\left\|T_{\alpha, \beta} \vec{f}\right\|_{R_{\beta}\left(\partial \Omega^{\beta}\right)}^{2} \lesssim\left\|T_{\alpha, \beta} \vec{f}\right\|_{L_{1,1 / 2}^{2}\left(\partial \Omega^{\beta}\right)}^{2}+\int_{-\infty}^{\infty} \int_{\partial \Omega_{t}^{\beta}}\left|S_{t}^{\beta}\left(\left\langle\frac{\partial T_{\alpha, \beta} \vec{f}}{\partial t}, N_{t}^{\beta}\right\rangle\right)\right|^{2} d \sigma_{t}^{\beta} d t .
$$

Above we have proved that,

$$
\left\|T_{\alpha, \beta} \vec{f}\right\|_{L_{1,1 / 2}^{2}\left(\partial \Omega^{\beta}\right)}^{2} \leq C(\alpha-\beta)\|\vec{f}\|_{L^{2}\left(\partial \Omega^{\alpha}\right)},
$$

where $C$ is a constant independent of $\alpha$ and $\beta$. We have also derived a decomposition

$$
S_{t}^{\beta}\left(\left\langle\frac{\partial T_{\alpha, \beta} \vec{f}}{\partial t}, N_{t}^{\beta}\right\rangle\right)=I_{1}-I_{21}-I_{22},
$$

and we have proved that

$$
\int_{-\infty}^{\infty} \int_{\mathbb{R}^{n-1}}\left(I_{22} \circ \rho_{\beta}(x, t)\right)^{2} d x d t \leq C(\alpha-\beta)\|\vec{f}\|_{L^{2}\left(\partial \Omega^{\alpha}\right)}^{2} .
$$

We therefore just have to focus on $I_{1}-I_{21}$. Let $\vec{v}=S_{\beta}\left(\vec{f} \circ \rho_{\alpha} \circ \pi_{\beta}\right) . p^{\beta}$ and $p^{\alpha}$ be the pressures associated to $S_{\beta}\left(\vec{f} \circ \rho_{\alpha} \circ \pi_{\beta}\right)$ and $S_{\alpha} \vec{f}$ respectively. We have derived the following formula for this difference.

$$
\begin{aligned}
& I_{1} \circ \rho_{\beta}(y, t)-I_{21} \circ \rho_{\beta}(y, t)= \\
& -\sum_{j, k=1}^{n-1} \int_{\mathbb{R}^{n-1}} \beta\left[\beta^{2} \frac{\partial P_{\gamma \epsilon} A}{\partial x_{j}} \frac{P_{\gamma \epsilon} A(y, t)-P_{\gamma \epsilon} A(z, t)}{w_{n}|K(\beta, y, z, t)|^{n}}+\frac{y_{j}-z_{j}}{w_{n}|K(\beta, y, z, t)|^{n}}\right] \\
& \times \frac{\partial P_{\gamma \epsilon} A}{\partial x_{k}} \frac{\partial v^{k}}{\partial x_{j}} \circ \rho_{\beta}(z, t) d z \\
& +\sum_{j, k=1_{\mathbb{R}^{n-1}}}^{n-1} \alpha\left[\beta^{2} \frac{\partial P_{\gamma \epsilon} A}{\partial x_{j}} \frac{P_{\gamma \epsilon} A(y, t)-P_{\gamma \epsilon} A(z, t)}{w_{n}|K(\beta, y, z, t)|^{n}}+\frac{y_{j}-z_{j}}{w_{n}|K(\beta, y, z, t)|^{n}}\right] \\
& \times \frac{\partial P_{\gamma \epsilon} A}{\partial x_{k}} \frac{\partial\left(S_{\alpha} \vec{f}\right)^{k}}{\partial x_{j}} \circ \rho_{\alpha}(z, t) d z
\end{aligned}
$$




$$
\begin{aligned}
& +\sum_{j, k=1}^{n-1} \int_{\mathbb{R}^{n-1}} \beta\left[\beta^{2} \frac{\partial P_{\gamma \epsilon} A}{\partial x_{k}} \frac{P_{\gamma \epsilon} A(y, t)-P_{\gamma \epsilon} A(z, t)}{w_{n}|K(\beta, y, z, t)|^{n}}+\frac{y_{k}-z_{k}}{w_{n}|K(\beta, y, z, t)|^{n}}\right] \\
& \times \frac{\partial P_{\gamma \epsilon} A}{\partial x_{j}} \frac{\partial v^{k}}{\partial x_{j}} \circ \rho_{\beta}(z, t) d z \\
& -\sum_{j, k=1}^{n-1} \int_{\mathbb{R}^{n-1}} \alpha\left[\beta^{2} \frac{\partial P_{\gamma \epsilon} A}{\partial x_{k}} \frac{P_{\gamma \epsilon} A(y, t)-P_{\gamma \epsilon} A(z, t)}{w_{n}|K(\beta, y, z, t)|^{n}}+\frac{y_{k}-z_{k}}{w_{n}|K(\beta, y, z, t)|^{n}}\right] \\
& \times \frac{\partial P_{\gamma \epsilon} A}{\partial x_{j}} \frac{\partial\left(S_{\alpha} \vec{f}\right)^{k}}{\partial x_{j}} \circ \rho_{\alpha}(z, t) d z \\
& +\sum_{j=1}^{n-1} \int_{\mathbb{R}^{n-1}}\left[\beta^{2} \frac{\partial P_{\gamma \epsilon} A}{\partial x_{j}} \frac{P_{\gamma \epsilon} A(y, t)-P_{\gamma \epsilon} A(z, t)}{w_{n}|K(\beta, y, z, t)|^{n}}+\frac{y_{j}-z_{j}}{w_{n}|K(\beta, y, z, t)|^{n}}\right] \\
& \times\left(\frac{\partial v^{0}}{\partial x_{j}}\right) \circ \rho_{\beta}(z, t) d z \\
& -\sum_{j=1}^{n-1} \int_{\mathbb{R}^{n-1}}\left[\beta^{2} \frac{\partial P_{\gamma \epsilon} A}{\partial x_{j}} \frac{P_{\gamma \epsilon} A(y, t)-P_{\gamma \epsilon} A(z, t)}{w_{n}|K(\beta, y, z, t)|^{n}}+\frac{y_{j}-z_{j}}{w_{n}|K(\beta, y, z, t)|^{n}}\right] \\
& \times\left(\frac{\partial\left(S_{\alpha} \vec{f}\right)^{0}}{\partial x_{j}}\right) \circ \rho_{\alpha}(z, t) d z \\
& -\sum_{k=1}^{n-1} \int_{\mathbb{R}^{n-1}}\left[\beta^{2} \frac{\partial P_{\gamma \epsilon} A}{\partial x_{k}} \frac{P_{\gamma \epsilon} A(y, t)-P_{\gamma \epsilon} A(z, t)}{w_{n}|K(\beta, y, z, t)|^{n}}+\frac{y_{k}-z_{k}}{w_{n}|K(\beta, y, z, t)|^{n}}\right] \\
& \times\left(\frac{\partial v^{k}}{\partial x_{0}}\right) \circ \rho_{\beta}(z, t) d z \\
& +\sum_{k=1}^{n-1} \int_{\mathbb{R}^{n-1}}\left[\beta^{2} \frac{\partial P_{\gamma \epsilon} A}{\partial x_{k}} \frac{P_{\gamma \epsilon} A(y, t)-P_{\gamma \epsilon} A(z, t)}{w_{n}|K(\beta, y, z, t)|^{n}}+\frac{y_{k}-z_{k}}{w_{n}|K(\beta, y, z, t)|^{n}}\right] \\
& \times\left(\frac{\partial\left(S_{\alpha} \vec{f}\right)^{k}}{\partial x_{0}}\right) \circ \rho_{\alpha}(z, t) d z \\
& -\frac{1}{2} p^{\beta} \circ \rho_{\beta}(y, t) \\
& +\int_{\mathbb{R}^{n-1}} \frac{\left[\beta \nabla P_{\gamma \epsilon} A \cdot(y-z)-\beta\left(P_{\gamma \epsilon} A(y, t)-P_{\gamma \epsilon} A(z, t)\right)\right]}{w_{n}|K(\beta, y, z, t)|^{n}} p^{\beta} \circ \rho_{\beta}(z, t) d z \\
& +\frac{1}{2} p^{\alpha} \circ \rho_{\beta}(y, t) \\
& -\int_{\mathbb{R}^{n-1}} \frac{\left[\alpha \nabla P_{\gamma \epsilon} A \cdot(y-z)-\beta\left(P_{\gamma \epsilon} A(y, t)-P_{\gamma \epsilon} A(z, t)\right)\right]}{w_{n} \mid\left(\left.K(\beta, y, z, t)\right|^{n}\right.} p^{\alpha} \circ \rho_{\alpha}(z, t) d z .
\end{aligned}
$$


Introduce the following notation,

$$
A_{j}(\beta, y, z, t)=\left[\beta^{2} \frac{\partial P_{\gamma \epsilon} A}{\partial x_{j}} \frac{P_{\gamma \epsilon} A(y, t)-P_{\gamma \epsilon} A(z, t)}{w_{n}|K(\beta, y, z, t)|^{n}}+\frac{y_{j}-z_{j}}{w_{n}|K(\beta, y, z, t)|^{n}}\right] .
$$

Using this and summation convention we can rewrite the formula above in a shorter form,

$$
\begin{aligned}
& I_{1} \circ \rho_{\beta}(y, t)-I_{21} \circ \rho_{\beta}(y, t)= \\
& \int_{\mathbb{R}^{n-1}} \alpha A_{j}(\beta, y, z, t) \frac{\partial P_{\gamma \epsilon} A}{\partial x_{k}} \frac{\partial\left(S_{\alpha} \vec{f}\right)^{k}}{\partial x_{j}} \circ \rho_{\alpha}(z, t)-\beta A_{j}(\beta, y, z, t) \frac{\partial P_{\gamma \epsilon} A}{\partial x_{k}} \frac{\partial v^{k}}{\partial x_{j}} \circ \rho_{\beta}(z, t) d z \\
+ & \int_{\mathbb{R}^{n-1}} \beta A_{k}(\beta, y, z, t) \frac{\partial P_{\gamma \epsilon} A}{\partial x_{j}} \frac{\partial v^{k}}{\partial x_{j}} \circ \rho_{\beta}(z, t)-\alpha A_{k}(\beta, y, z, t) \frac{\partial P_{\gamma \epsilon} A}{\partial x_{j}} \frac{\partial\left(S_{\alpha} \vec{f}\right)^{k}}{\partial x_{j}} \circ \rho_{\alpha}(z, t) d z \\
+ & \int_{\mathbb{R}^{n-1}} A_{j}(\beta, y, z, t)\left(\frac{\partial v^{0}}{\partial x_{j}}\right) \circ \rho_{\beta}(z, t)-A_{j}(\beta, y, z, t)\left(\frac{\partial\left(S_{\alpha} \vec{f}\right)^{0}}{\partial x_{j}}\right) \circ \rho_{\alpha}(z, t) d z \\
+ & \int_{\mathbb{R}^{n-1}} A_{k}(\beta, y, z, t)\left(\frac{\partial\left(S_{\alpha} \vec{f}\right)^{k}}{\partial x_{0}}\right) \circ \rho_{\alpha}(z, t)-A A_{k}(\beta, y, z, t)\left(\frac{\partial v^{k}}{\partial x_{0}}\right) \circ \rho_{\beta}(z, t) d z \\
- & \frac{1}{2} p^{\beta} \circ \rho_{\beta}(y, t)+\int_{\mathbb{R}^{n-1}} \frac{\left[\beta \nabla P_{\gamma \epsilon} A \cdot(y-z)-\beta\left(P_{\gamma \epsilon} A(y, t)-P_{\gamma \epsilon} A(z, t)\right)\right]}{w_{n}|K(\beta, y, z, t)|^{n}} p^{\beta} \circ \rho_{\beta}(z, t) d z \\
+ & \frac{1}{2} p^{\alpha} \circ \rho_{\beta}(y, t)-\int_{\mathbb{R}^{n-1}} \frac{\left[\alpha \nabla P_{\gamma \epsilon} A \cdot(y-z)-\beta\left(P_{\gamma \epsilon} A(y, t)-P_{\gamma \epsilon} A(z, t)\right)\right]}{\alpha, z, t)\left.\right|^{n} \circ \rho_{\alpha}(z, t) d z .}
\end{aligned}
$$

We need to estimate $I_{1} \circ \rho_{\beta}(y, t)-I_{21} \circ \rho_{\beta}(y, t)$ in $L^{2}\left(\mathbb{R}^{n}, d y d t\right)$. Obviously in order to do so we only have to consider the following operators for relevant indicies.

$$
\begin{aligned}
T_{1} \vec{f}(y, t) & =\int_{\mathbb{R}^{n-1}}\left[\alpha \frac{\partial\left(S_{\alpha} \vec{f}\right)^{k}}{\partial x_{j}} \circ \rho_{\alpha}(z, t)-\beta \frac{\partial v^{k}}{\partial x_{j}} \circ \rho_{\beta}(z, t)\right] A_{j}(\beta, y, z, t) \frac{\partial P_{\gamma \epsilon} A}{\partial x_{k}} d z \\
T_{2} \vec{f}(y, t) & =\int_{\mathbb{R}^{n-1}} A_{k}(\beta, y, z, t)\left[\left(\frac{\partial v^{m}}{\partial x_{j}}\right) \circ \rho_{\beta}(z, t)-\left(\frac{\partial\left(S_{\alpha} \vec{f}\right)^{m}}{\partial x_{j}}\right) \circ \rho_{\alpha}(z, t)\right] d z \\
T_{3} \vec{f}(y, t) & =\frac{1}{2}\left[p^{\alpha} \circ \rho_{\beta}(y, t)-p^{\beta} \circ \rho_{\beta}(y, t)\right] \\
T_{4} \vec{f}(y, t) & =\int_{\mathbb{R}^{n-1}} \frac{\left[\beta \nabla P_{\gamma \epsilon} A \cdot(y-z)-\beta\left(P_{\gamma \epsilon} A(y, t)-P_{\gamma \epsilon} A(z, t)\right)\right]}{w_{n}|K(\beta, y, z, t)|^{n}} p^{\beta} \circ \rho_{\beta}(z, t) d z \\
& -\int_{\mathbb{R}^{n-1}} \frac{\left[\alpha \nabla P_{\gamma \epsilon} A \cdot(y-z)-\beta\left(P_{\gamma \epsilon} A(y, t)-P_{\gamma \epsilon} A(z, t)\right)\right]}{w_{n} \mid\left(\left.K(\beta, y, z, t)\right|^{n}\right.} p^{\alpha} \circ \rho_{\alpha}(z, t) d z .
\end{aligned}
$$


We will now treat each of these operators. Rewriting $T_{1} \vec{f}$ we get,

$$
\begin{aligned}
T_{1} \vec{f}(y, t) & =\int_{\mathbb{R}^{n-1}}\left[(\alpha-\beta) A_{j}(\beta, y, z, t) \frac{\partial\left(S_{\alpha} \vec{f}\right)^{k}}{\partial x_{j}} \circ \rho_{\alpha}(z, t)\right] \frac{\partial P_{\gamma \epsilon} A}{\partial x_{k}} d z \\
& +\int_{\mathbb{R}^{n-1}} \beta A_{j}(\beta, y, z, t)\left[\frac{\partial\left(S_{\alpha} \vec{f}\right)^{k}}{\partial x_{j}} \circ \rho_{\alpha}(z, t)-\frac{\partial v^{k}}{\partial x_{j}} \circ \rho_{\beta}(z, t)\right] \frac{\partial P_{\gamma \epsilon} A}{\partial x_{k}} d z \\
& :=T_{11} \vec{f}(y, t)+T_{12} \vec{f}(y, t) .
\end{aligned}
$$

As $T_{11}$ essentially is an elliptic singular integral operator of the type considered by Coifman-Macintosh and Meyer[5] we deduce using the usual nontangential estimate that

$$
\left\|T_{11} \vec{f}\right\|_{L^{2}\left(\mathbb{R}^{n}, d y d t\right)} \leq C(\alpha-\beta)\|\vec{f}\|_{L^{2}\left(\partial \Omega^{\alpha}\right)} .
$$

In the same way,

$$
\left\|T_{12} \vec{f}\right\|_{L^{2}\left(\mathbb{R}^{n}, d y d t\right)}^{2} \leq C \int_{-\infty}^{\infty} \int_{\mathbb{R}^{n-1}}\left|\frac{\partial\left(S_{\alpha} \vec{f}\right)^{k}}{\partial x_{j}} \circ \rho_{\alpha}(z, t)-\frac{\partial v^{k}}{\partial x_{j}} \circ \rho_{\beta}(z, t)\right|^{2} d z d t .
$$

But,

$$
\begin{aligned}
\frac{\partial\left(S_{\alpha} \vec{f}\right)^{k}}{\partial x_{j}} \circ \rho_{\alpha}(z, t) & =\sum_{i=0}^{n-1} \int_{-\infty}^{\infty} \int_{\mathbb{R}^{n-1}} \Gamma_{x_{j}}^{i k}\left(\alpha\left(P_{\gamma \epsilon} A(z, t)-P_{\gamma \epsilon} A(w, s)\right), z-w, t-s\right) \\
& \times f_{i} \circ \rho_{\alpha}(w, s) \sqrt{1+\alpha^{2}\left|\nabla P_{\gamma \epsilon} A(w, s)\right|^{2}} d w d s \\
\frac{\partial v^{k}}{\partial x_{j}} \circ \rho_{\beta}(z, t) & =\sum_{i=0}^{n-1} \int_{-\infty}^{\infty} \int_{\mathbb{R}^{n-1}} \Gamma_{x_{j}}^{i k}\left(\beta\left(P_{\gamma \epsilon} A(z, t)-P_{\gamma \epsilon} A(w, s)\right), z-w, t-s\right) \\
& \times f_{i} \circ \rho_{\alpha}(w, s) \sqrt{1+\beta^{2}\left|\nabla P_{\gamma \epsilon} A(w, s)\right|^{2}} d w d s
\end{aligned}
$$

Using the fundamental theorem of calculus we have,

$$
\begin{aligned}
& \Gamma_{x_{j}}^{i k}\left(\alpha\left(P_{\gamma \epsilon} A(z, t)-P_{\gamma \epsilon} A(w, s)\right), z-w, t-s\right) \\
- & \Gamma_{x_{j}}^{i k}\left(\beta\left(P_{\gamma \epsilon} A(z, t)-P_{\gamma \epsilon} A(w, s)\right), z-w, t-s\right) \\
= & \int_{\beta}^{\alpha} \Gamma_{x_{j} x_{0}}^{i k}\left(\sigma\left(P_{\gamma \epsilon} A(z, t)-P_{\gamma \epsilon} A(w, s)\right), z-w, t-s\right)\left(P_{\gamma \epsilon} A(z, t)-P_{\gamma \epsilon} A(w, s)\right) d \sigma
\end{aligned}
$$

Again using the results of Hofmann[18] on parabolic singular integrals we may conclude that,

$$
\int_{-\infty}^{\infty} \int_{\mathbb{R}^{n-1}}\left|\frac{\partial\left(S_{\alpha} \vec{f}\right)^{k}}{\partial x_{j}} \circ \rho_{\alpha}(z, t)-\frac{\partial v^{k}}{\partial x_{j}} \circ \rho_{\beta}(z, t)\right|^{2} d z d t \leq C(\alpha-\beta)\|\vec{f}\|_{L^{2}\left(\partial \Omega^{\alpha}\right)} .
$$

This completes the estimate of $T_{1} \vec{f}$. By a similar argument we see that $\left\|T_{2} \vec{f}\right\|_{L^{2}\left(\mathbb{R}^{n}, d y d t\right)} \leq C(\alpha-\beta)\|\vec{f}\|_{L^{2}\left(\Omega^{\alpha}\right)}$. Introducing, $\tilde{K}(\beta, \alpha, y, z, t)=\left(\beta P_{\gamma \epsilon} A(y, t)-\right.$ 

$\left.\alpha P_{\gamma \epsilon} A(z, t), y-z\right)$ and rewriting $T_{3} \vec{f}$ we get,

$$
\begin{aligned}
T_{3} \vec{f}(y, t)= & \frac{1}{2} \int_{\mathbb{R}^{n-1}} \frac{\left\langle\rho_{\beta}(y, t)-\rho_{\alpha}(z, t), \vec{f} \circ \rho_{\alpha}\right\rangle}{w_{n}|\tilde{K}(\beta, \alpha, y, z, t)|^{n}} \sqrt{1+\alpha^{2}\left|\nabla P_{\gamma \epsilon} A(z, t)\right|^{2}} d z \\
- & \frac{1}{2} \int_{\mathbb{R}^{n-1}} \frac{\left\langle\rho_{\beta}(y, t)-\rho_{\beta}(z, t), \vec{f} \circ \rho_{\alpha}\right\rangle}{w_{n}|K(\beta, y, z, t)|^{n}} \sqrt{1+\beta^{2}\left|\nabla P_{\gamma \epsilon} A(z, t)\right|^{2}} d z \\
= & \frac{1}{2} \int_{\mathbb{R}^{n-1}} \frac{\left\langle\rho_{\beta}(y, t)-\rho_{\alpha}(z, t), \vec{f} \circ \rho_{\alpha}\right\rangle}{w_{n}|\tilde{K}(\beta, \alpha, y, z, t)|^{n}} \\
& \times \frac{(\alpha-\beta)(\alpha+\beta)\left|\nabla P_{\gamma \epsilon} A(y, s)\right|^{2}}{\left[\sqrt{1+\beta^{2}\left|\nabla P_{\gamma \epsilon} A(y, s)\right|^{2}}+\sqrt{1+\alpha^{2}\left|\nabla P_{\gamma \epsilon} A(y, s)\right|^{2}}\right]} d z \\
+ & \frac{1}{2} \int_{\mathbb{R}^{n-1}} \frac{\left\langle\rho_{\beta}(z, t)-\rho_{\alpha}(z, t), \vec{f} \circ \rho_{\alpha}\right\rangle}{w_{n}|\tilde{K}(\beta, \alpha, y, z, t)|^{n}} \sqrt{1+\beta^{2}\left|\nabla P_{\gamma \epsilon} A(z, t)\right|^{2}} d z \\
+ & \frac{1}{2 w_{n}} \int_{\mathbb{R}^{n-1}}\left[\frac{1}{|\tilde{K}(\beta, \alpha, y, z, t)|^{n}}-\frac{1}{|K(\beta, y, z, t)|^{n}}\right] \\
& \quad\left\langle\rho_{\beta}(y, t)-\rho_{\beta}(z, t), \vec{f} \circ \rho_{\alpha}\right\rangle \sqrt{1+\beta^{2}\left|\nabla P_{\gamma \epsilon} A(z, t)\right|^{2}} d z \\
:= & T_{31} \vec{f}(y, t)+T_{32} \vec{f}(y, t)+T_{33} \vec{f}(y, t)
\end{aligned}
$$

Using the theory of elliptic singular integrals we immediately see that,

$$
\left\|T_{31} \vec{f}\right\|_{L^{2}\left(\mathbb{R}^{n}, d y d t\right)}+\left\|T_{32} \vec{f}\right\|_{L^{2}\left(\mathbb{R}^{n}, d y d t\right)} \leq C(\alpha-\beta)\|\vec{f}\|_{L^{2}\left(\Omega^{\alpha}\right)} .
$$

After some thought we realize that the same is true for the integral of $T_{33} \vec{f}$. Rewriting $T_{4} \vec{f}$ we get,

$$
\begin{aligned}
& T_{4} \vec{f}(y, t) \\
= & \int_{\mathbb{R}^{n-1}} \frac{\left[(\beta-\alpha) \nabla P_{\gamma \epsilon} A \cdot(y-z)\right]}{w_{n}|K(\beta, y, z, t)|^{n}} p^{\beta} \circ \rho_{\beta}(z, t) d z \\
- & \int_{\mathbb{R}^{n-1}} \frac{\left[\alpha \nabla P_{\gamma \epsilon} A \cdot(y-z)-\beta\left(P_{\gamma \epsilon} A(y, t)-P_{\gamma \epsilon} A(z, t)\right)\right]}{w_{n} \mid\left(\left.K(\beta, y, z, t)\right|^{n}\right.}\left[p^{\beta} \circ \rho_{\beta}-p^{\alpha} \circ \rho_{\alpha}\right](z, t) d z \\
:= & T_{41} \vec{f}(y, t)+T_{42} \vec{f}(y, t)
\end{aligned}
$$

Again by elliptic theory, $\left\|T_{41} \vec{f}\right\|_{L^{2}\left(\mathbb{R}^{n}, d y d t\right)} \leq C(\alpha-\beta)\|\vec{f}\|_{L^{2}\left(\partial \Omega^{\alpha}\right)}$. Also by elliptic singular integrals we get,

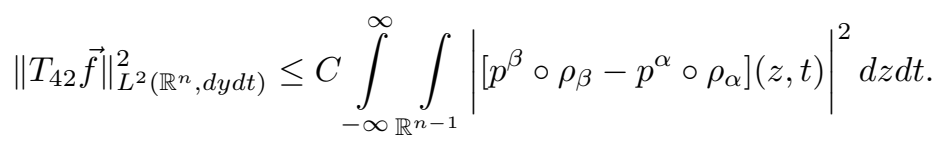


Now,

$$
\begin{aligned}
& {\left[p^{\beta} \circ \rho_{\beta}-p^{\alpha} \circ \rho_{\alpha}\right](z, t) } \\
= & \int_{\mathbb{R}^{n-1}} \frac{\left\langle\rho_{\beta}(y, t)-\rho_{\beta}(z, t), \vec{f} \circ \rho_{\alpha}\right\rangle}{w_{n} \mid\left(\left.K(\beta, y, z, t)\right|^{n}\right.} \sqrt{1+\beta^{2}\left|\nabla P_{\gamma \epsilon} A(z, t)\right|^{2}} d z \\
- & \int_{\mathbb{R}^{n-1}} \frac{\left\langle\rho_{\alpha}(y, t)-\rho_{\alpha}(z, t), \vec{f} \circ \rho_{\alpha}\right\rangle}{w_{n} \mid\left(\left.K(\alpha, y, z, t)\right|^{n}\right.} \sqrt{1+\alpha^{2}\left|\nabla P_{\gamma \epsilon} A(z, t)\right|^{2}} d z \\
= & \int_{\mathbb{R}^{n-1}} \frac{\left\langle\rho_{\beta}(y, t)-\rho_{\beta}(z, t), \vec{f} \circ \rho_{\alpha}\right\rangle}{w_{n} \mid\left(\left.K(\beta, y, z, t)\right|^{n}\right.} \frac{(\beta-\alpha)(\alpha+\beta)\left|\nabla P_{\gamma \epsilon} A(y, s)\right|^{2}}{\left[\sqrt{1+\beta^{2}\left|\nabla P_{\gamma \epsilon} A(y, s)\right|^{2}}+\sqrt{1+\alpha^{2}\left|\nabla P_{\gamma \epsilon} A(y, s)\right|^{2}}\right]} d z \\
+ & \left.\int_{\mathbb{R}^{n-1}} \frac{\left\langle\left((\alpha-\beta)\left(P_{\gamma \epsilon}(y, t)-P_{\gamma \epsilon}(z, t)\right), 0\right), \vec{f} \circ \rho_{\alpha}\right\rangle}{w_{n} \mid\left(\left.K(\beta, y, z, t)\right|^{n}\right.} \sqrt{1+\alpha^{2}\left|\nabla P_{\gamma \epsilon} A(z, t)\right|^{2}} d z\right] \\
+ & \frac{1}{w_{n}} \int_{\mathbb{R}^{n-1}}\left[\frac{1}{\mid\left(\left.K(\beta, y, z, t)\right|^{n}\right.}-\frac{1\left(\left.K(\alpha, y, z, t)\right|^{n}\right.}{\mid(K)} d z\right. \\
& \times\left\langle\rho_{\alpha}(y, t)-\rho_{\alpha}(z, t), \vec{f} \circ \rho_{\alpha}\right\rangle \sqrt{1+\alpha^{2}\left|\nabla P_{\gamma \epsilon} A(z, t)\right|^{2}} d z \vec{f} .
\end{aligned}
$$

Obviously

$$
\left\|T_{421} \vec{f}\right\|_{L^{2}\left(\mathbb{R}^{n}, d y d t\right)}+\left\|T_{422} \vec{f}\right\|_{L^{2}\left(\mathbb{R}^{n}, d y d t\right)} \leq C(\alpha-\beta)\|\vec{f}\|_{L^{2}\left(\partial \Omega^{\alpha}\right)} .
$$

That the same estimate is true for the term determined by $T_{423}$ follows from elliptic theory. Adding our estimates we may conclude that

$$
\int_{-\infty}^{\infty} \int_{\mathbb{R}^{n-1}}\left[I_{1} \circ \rho_{\beta}(y, t)-I_{21} \circ \rho_{\beta}(y, t)\right]^{2} d y d t \leq C(\alpha-\beta)\|\vec{f}\|_{L^{2}\left(\partial \Omega^{\alpha}\right)}^{2} .
$$

Looking back and adding estimates we have proved that,

$$
\left\|T_{\alpha, \beta} \vec{f}\right\|_{R_{\beta}\left(\partial \Omega^{\beta}\right)} \leq C(\alpha-\beta)\|\vec{f}\|_{L^{2}\left(\partial \Omega^{\alpha}\right)}
$$

where $C$ is a constant independent of $\alpha$ and $\beta$ and

$$
T_{\alpha, \beta} \vec{f}=S_{\beta}\left(\vec{f} \circ \rho_{\alpha} \circ \pi_{\beta}\right)-A_{\alpha, \beta} S_{\alpha} \vec{f} \circ \rho_{\alpha} \circ \pi_{\beta} .
$$

By construction we formally have $\vec{u}_{0}=\sum_{k=1}^{\infty} S_{\alpha} \vec{f}_{k}$ and our main objective was to prove that $\sum_{k=1}^{\infty} \overrightarrow{f_{k}}$ converges in $L^{2}\left(\partial \Omega^{\alpha}\right)$. But the estimate we have proved implies that,

$$
\left\|\vec{f}_{k}\right\|_{L^{2}\left(\partial \Omega^{\alpha}\right)} \leq C^{k}(\alpha-\beta)^{k}\left\|\vec{f}_{0}\right\|_{L^{2}\left(\partial \Omega^{\alpha}\right)}
$$

If $(\alpha-\beta) \leq C^{-1} / 2$ we may therefore conclude that we have the desired convergence.

As mentioned in the beginning of the proof one may prove, in an argument similar to the one presented above, that $S_{\beta}^{-1}$ exists if $\beta$ is small enough by considering $\partial \Omega^{\beta}$ as a perturbation of the halfplane $\left\{\left(x_{0}, x, t\right): x_{0}>0\right\}$. We have therefore completed our continuity argument and therefore the proof of Theorem 9.3. 
10. Proof of uniqueness. In this section we prove the uniqueness statements of Theorem A, B and C.

Proof of uniqueness in the Dirichlet Problem. Assume that $\vec{u}$ is a weak solution of the linearized Navier-Stokes equations and assume that

(i) $\quad \tilde{N}_{*, e}(\vec{u}) \in L^{2}(\partial \Omega)$

(ii) $\lim _{\left(y_{0}, y, t\right) \rightarrow\left(x_{0}, x, t\right)} \vec{u}\left(y_{0}, y, t\right)=\overrightarrow{0} \quad$ non-tangentially for almost every $\mathrm{t}$ and for $\operatorname{almost} \operatorname{every}\left(x_{0}, x, t\right) \in \partial \Omega_{t}$.

We want to prove that $\vec{u} \equiv \overrightarrow{0}$. As $\left\|\mathbb{D}_{n} P_{\gamma \epsilon} A\right\|_{*} \leq c\left\|\mathbb{D}_{n} A\right\|_{*}$ and $\left\|P_{\gamma \epsilon} A\right\|_{\text {comm }} \leq$ $c\|A\|_{\text {comm }}$ where $c$ is a constant independent of $\epsilon$ the existence results of the previous section are valid on $\Omega^{\epsilon}$. Furthermore the inequalities of the previous sections are valid on $\Omega^{\epsilon}$ with constants independent of $\epsilon$. In addition to this $\Omega^{\epsilon}$ is a $C^{\infty}$-smooth domain.

Let $\vec{u}_{\epsilon}$ be the weak solution(given by the existence proof) to the Dirichlet problem in $\Omega^{\epsilon}$ with data on $\partial \Omega^{\epsilon}$ given by the restriction of $\vec{u}$ to $\partial \Omega^{\epsilon}$. As $\tilde{N}_{*, e}(\vec{u}) \in L^{2}(\partial \Omega)$ this restriction is well defined. Then $\vec{u}_{\epsilon}=D_{\epsilon} \vec{f}_{\epsilon}$ for some $\vec{f}_{\epsilon} \in L^{2}\left(\partial \Omega^{\epsilon}\right)$ where $D_{\epsilon}$ is the double layer potential operator on $\Omega^{\epsilon}$. This follows from the existence results of the previous section as $\tilde{N}_{*, e}(\vec{u}) \in L^{2}(\partial \Omega)$.

We now claim that $\vec{u}_{\epsilon} \equiv \vec{u}$ in $\Omega^{\epsilon}$ for all $\epsilon>0$. Let us assume the claim for now and let $\tilde{N}_{*}^{\epsilon}$ be the non tangential maximal function operator on $\Omega^{\epsilon}$. Similarly define $\tilde{N}_{*, e}^{\epsilon}$ Then trivially, $\tilde{N}_{*, e}^{\epsilon}(\vec{u}) \leq \tilde{N}_{*, e}(\vec{u})$. By the existence results in $\Omega^{\epsilon}$ we have that the following inequality is valid with a constant $c$ independent of $\epsilon$ :

$$
\left\|\tilde{N}_{*, e}^{\epsilon}(\vec{u})\right\|_{L^{2}\left(\partial \Omega^{\epsilon}\right)} \leq c\|\vec{u}\|_{L^{2}\left(\partial \Omega^{\epsilon}\right)} .
$$

As $\tilde{N}_{*, e}(\vec{u}) \in L^{2}\left(\partial \Omega_{t}\right)$ for almost every $t$ we get by dominated convergence that $\tilde{N}_{*, e}(\vec{u})=0$ a.e on $\partial \Omega$. I.e. $\tilde{N}_{*, e}^{\epsilon}(\vec{u}) \equiv 0$ for every $\epsilon$ and almost every $t$. I.e., $\vec{u} \equiv 0$.

Left is now to prove the claim. Let $\vec{w}_{\epsilon}:=\vec{u}_{\epsilon}-\vec{u}$ in the closure of $\Omega^{\epsilon}$. Then $\vec{w}_{\epsilon}$ is a weak solution in $\Omega^{\epsilon}$ and $\vec{w}_{\epsilon}$ is zero on the boundary of $\Omega^{\epsilon}$. We want to prove that $\vec{w}_{\epsilon} \equiv \overrightarrow{0}$. To prove this it is enough to prove that for all $\vec{F} \in C_{0}^{\infty}\left(\Omega^{\epsilon}\right)$ the following is true,

$$
\int_{\Omega^{\epsilon}}\left\langle\vec{w}_{\epsilon}, \vec{F}\right\rangle d Y d s=0 .
$$

I.e., given $\vec{F} \in C_{0}^{\infty}\left(\Omega^{\epsilon}\right)$ we want to prove that there exist an admissible pair of test functions $(\vec{\phi}, q)$ in $\Omega^{\epsilon}$ such that

$$
\vec{\phi}_{s}+\Delta \vec{\phi}+\nabla q=\vec{F} .
$$

Let $\left(\vec{\phi}^{1}, q_{1}\right)$ solve the problem $\vec{\phi}_{s}^{1}+\Delta \vec{\phi}^{1}+\nabla q_{1}=\vec{F}, \quad \operatorname{div} \vec{\phi}^{1}=0$ in the whole of $\mathbb{R}^{n}$. We postpone the proof of the following lemma for now.

LEMMA 10.1. The restriction of $\vec{\phi}^{1}$ to $\partial \Omega^{\epsilon}$ is in the space $R\left(\partial \Omega^{\epsilon}\right)$.

We now consider the problem, $\vec{\phi}_{s}^{2}+\Delta \vec{\phi}^{2}+\nabla q_{2}=\vec{F}, \quad \operatorname{div} \vec{\phi}^{2}=0$ in $\Omega^{\epsilon}$ with boundary data $\vec{\phi}^{2}=\vec{\phi}^{1}$. Using lemma 10.1 we notice that we are solving the Dirichlet problem with data in our regularity space $R\left(\partial \Omega^{\epsilon}\right)$. Using our existence result from 
the previous section we may conclude that $\vec{\phi}^{2}$ can be represented using the single layer potential. Define $\vec{\psi}=\vec{\phi}^{1}-\vec{\phi}^{2}, q=q_{1}-q_{2}$. Then $(\vec{\psi}, q)$ is an admissible pair of test functions in $\Omega^{\epsilon}$ reproducing $\vec{F} \in C_{0}^{\infty}\left(\Omega^{\epsilon}\right)$.

This completes the proof of uniqueness in the Dirichlet problem modulo the lemma.

Proof Lemma 10.1. We have that

$$
\begin{aligned}
& \vec{\phi}^{1}(X, t)=\int_{-\infty}^{t} \int_{\mathbb{R}^{n}} \Gamma(X-Y, t-s) \vec{F}(Y, s) d Y d s, \\
& q_{1}(X, t)=\int_{\mathbb{R}^{n}} \frac{\langle X-Q, \vec{F}(Y, t)\rangle}{w_{n}|X-Y|^{n}} d Y .
\end{aligned}
$$

We want to verify that the restriction of $\vec{\phi}^{1}$ to $\partial \Omega^{\epsilon}$ is in $R\left(\partial \Omega^{\epsilon}\right)$. Let $\Theta_{\epsilon}(\lambda, y, s)=$ $\left(\lambda+\epsilon+P_{\gamma \epsilon} A(y, s), y, s\right)$. Then as the support of $\vec{F}$ is contained in $\Omega^{\epsilon}$,

$$
\begin{aligned}
\vec{\phi}^{1}(X, t) & =\int_{-\infty}^{\infty} \int_{-\infty}^{t} \int_{\mathbb{R}^{n-1}} \Gamma\left((X, t)-\Theta_{\epsilon}(\lambda, y, s)\right) \vec{F} \circ \Theta_{\epsilon}(\lambda, y, s) d y d s d \lambda \\
& =\int_{0}^{\lambda(\vec{F})} \int_{-\infty}^{t} \int_{\mathbb{R}^{n-1}} \Gamma\left((X, t)-\Theta_{\epsilon}(\lambda, y, s)\right) \vec{F} \circ \Theta_{\epsilon}(\lambda, y, s) d y d s d \lambda \\
& =\int_{0}^{\lambda(\vec{F})} S_{\lambda, \epsilon} \vec{F}(X, t) d \lambda .
\end{aligned}
$$

Here $S_{\lambda, \epsilon} \vec{F}$ is the single layer potential with $\left\{\left(x_{0}, x, t\right): x_{0}=\epsilon+\lambda+P_{\gamma \epsilon} A(x, t)\right\}$ as the underlying boundary. Now using the estimates of maximal functions outlined in Section 2 as well as the arguments of Section 7 we may conclude that indeed the restriction of $\vec{\phi}^{1}$ to $\partial \Omega^{\epsilon}$ is in $R\left(\partial \Omega^{\epsilon}\right)$.

Proof of Uniqueness in the regularity problem. We assume that $\vec{u}=\overrightarrow{0}$ in $R\left(\partial \Omega^{\epsilon_{0}}\right)$, $\tilde{N}_{*}(\nabla \vec{u}), \tilde{N}_{*, e}(p) \in L^{2}\left(\partial \Omega^{\epsilon_{0}}\right)$ and that $(\vec{u}, p)$ solves the equation under consideration. We want to prove that $\vec{u} \equiv \overrightarrow{0}$. Fix $\left(X^{\prime}, t^{\prime}\right) \in \partial \Omega^{\epsilon_{0}}$ and $(X, t) \in \Omega^{\epsilon_{0}}$. Choose $r>0$ so large and $\epsilon>\epsilon_{0}$ so small that $(X, t) \in \Omega^{\epsilon} \cap Q_{r}\left(X^{\prime}, t^{\prime}\right)$ with

$$
Q_{r}\left(X^{\prime}, t^{\prime}\right):=\left\{\left(y_{0}, y, s\right):\left|t^{\prime}-s\right|<r^{2},\left|x_{0}^{\prime}-y_{0}\right|+\left|x^{\prime}-y\right|<r\right\} .
$$

Let $\vec{w}=\vec{u} \phi$ where $\phi \in C_{0}^{\infty}\left(Q_{2 r}\left(X^{\prime}, t^{\prime}\right)\right)$ is such that $\phi \equiv 1$ on the closure of $Q_{3 r / 2}\left(X^{\prime}, t^{\prime}\right)$. We furthermore assume that

$$
\left\|\phi_{t}\right\|_{\infty}+\sum_{i=0}^{n-1}\left\|\phi_{x_{i}}\right\|_{\infty}+\sum_{i, j=0}^{n-1}\left\|\phi_{x_{i} x_{j}}\right\|_{\infty} \leq c r^{-2} .
$$

Let $\delta \in(0,1)$ and let $s \in(-\infty, t-\delta)$. Let $\Gamma(Y, s)=\Gamma(X-Y, t-s)$ be the matrix of fundamental solutions. Let $\Gamma_{i}$ be the $i$-te row in that matrix. The vector $\Gamma_{i}$ solves the adjoint system of linearized Navier-Stokes equations with pressure $p=q^{i}$ according 
to Section 2. Using integration by parts we have,

$$
\begin{aligned}
I_{\epsilon, \delta}^{k}:= & \int_{-\infty}^{t-\delta} \int_{\Omega_{s}^{\epsilon}}\left(w^{k} \Gamma_{i k}\right)_{s} d Y d s \\
= & \int_{-\infty}^{t-\delta} \int_{\Omega_{s}^{\epsilon}} \frac{\partial \phi}{\partial s} u^{k} \Gamma_{i k} d Y d s+\int_{-\infty}^{t-\delta} \int_{\Omega_{s}^{\epsilon}}\left(\frac{\partial u^{k}}{\partial s} \phi \Gamma_{i k}+\frac{\partial \Gamma_{i k}}{\partial s} u^{k} \phi\right) d Y d s \\
= & \int_{-\infty}^{t-\delta} \int_{\Omega_{s}^{\epsilon}} \frac{\partial \phi}{\partial s} u^{k} \Gamma_{i k} d Y d s+\int_{-\infty}^{t-\delta} \int_{\Omega_{s}^{\epsilon}}\left(\Delta u^{k} \phi \Gamma_{i k}-\frac{\partial p}{\partial x_{k}} \phi \Gamma_{i k}\right) d Y d s \\
& -\int_{-\infty}^{t-\delta} \int_{\Omega_{s}^{\epsilon}}\left(\Delta \Gamma_{i k} u^{k} \phi-\frac{\partial q^{i}}{\partial x_{k}} \phi u^{k}\right) d Y d s \\
= & \int_{-\infty}^{t-\delta} \int_{\Omega_{s}^{\epsilon}} \frac{\partial \phi}{\partial s} u^{k} \Gamma_{i k} d Y d s+\int_{-\infty}^{t-\delta} \int_{\partial \Omega_{s}^{\epsilon}}\left(\frac{\partial u^{k}}{\partial N_{s}^{\epsilon}} \phi \Gamma_{i k}-\frac{\partial \Gamma_{i k}}{\partial N_{s}^{\epsilon}} u^{k} \phi\right) d \sigma_{s}^{\epsilon} d s \\
+ & \int_{-\infty}^{t} \int_{\Omega_{s}^{\epsilon}}\left(\nabla u^{k} \nabla\left(\phi \Gamma_{i k}\right)-\nabla \Gamma_{i k} \nabla\left(u^{k} \phi\right)\right) d Y d s \\
+ & \int_{t-\infty} \int_{\Omega_{s}^{\epsilon}}\left(\frac{\partial q^{i}}{\partial x_{k}}\left(\phi u^{k}\right)-\frac{\partial p}{\partial x_{k}}\left(\phi \Gamma_{i k}\right)\right) d Y d s .
\end{aligned}
$$

But a simple calculation shows that,

$$
\nabla u^{k} \nabla\left(\phi \Gamma_{i k}\right)-\nabla \Gamma_{i k} \nabla\left(u^{k} \phi\right)=\nabla u^{k} \nabla \phi \Gamma_{i k}-\nabla \Gamma_{i k} \nabla \phi u^{k} .
$$

Furthermore,

$$
\operatorname{div}\left(q^{i} \phi \vec{u}\right)-\operatorname{div}\left(p \phi \Gamma_{i}\right)=\left\langle\nabla q^{i}, \phi \vec{u}\right\rangle+q^{i}\langle\nabla \phi, \vec{u}\rangle-\left\langle\nabla p, \phi \Gamma_{i}\right\rangle-p\left\langle\nabla \phi, \Gamma_{i}\right\rangle
$$

Noting that terms containing $q^{i}$ make no contribution to $I_{\epsilon, \delta}^{k}$ we may conclude that,

$$
\begin{aligned}
\sum_{k=0}^{n-1} I_{\epsilon, \delta}^{k} & :=\sum_{k=0}^{n-1} \int_{-\infty}^{t-\delta} \int_{\Omega_{s}^{\epsilon}} \frac{\partial \phi}{\partial s} u^{k} \Gamma_{i k} d Y d s \\
& +\sum_{k=0}^{n-1} \int_{-\infty}^{t-\delta} \int_{\partial \Omega_{s}^{\epsilon}}\left(\frac{\partial u^{k}}{\partial N_{s}^{\epsilon}} \phi \Gamma_{i k}-\frac{\partial \Gamma_{i k}}{\partial N_{s}^{\epsilon}} u^{k} \phi\right) d \sigma_{s}^{\epsilon} d s \\
& +\sum_{k=0}^{n-1} \int_{-\infty}^{t-\delta} \int_{\Omega_{s}^{\epsilon}}\left(\nabla u^{k} \nabla \phi \Gamma_{i k}-\nabla \Gamma_{i k} \nabla \phi u^{k}\right) d Y d s
\end{aligned}
$$




$$
\begin{aligned}
& -\sum_{k=0}^{n-1} \int_{-\infty}^{t-\delta} \int_{\partial \Omega_{s}^{\epsilon}} p \phi \Gamma_{i k} N_{s}^{\epsilon, k} d \sigma_{s}^{\epsilon} d s \\
& +\sum_{k=0}^{n-1} \int_{-\infty}^{t-\delta} \int_{\Omega_{s}^{\epsilon}} p \frac{\partial \phi}{\partial x_{k}} \Gamma_{i k} d Y d s \\
& :=\sum_{k=0}^{n-1} A_{k}+B_{k}+C_{k}+D_{k}+E_{k} .
\end{aligned}
$$

By standard arguments we get,

$$
\sum_{k=0}^{n-1}\left|A_{k}\right|+\left|C_{k}\right|+\left|E_{k}\right| \leq C r^{(1-n) / 2}\left(\left\|\tilde{N}_{*}(|\nabla \vec{u}|)\right\|_{2}+\left\|\tilde{N}_{*, e}(p)\right\|_{2}\right) \rightarrow 0
$$

as $r \rightarrow \infty$. Now consider the second term in the expression $B_{k}$, i.e.

$$
\int_{-\infty}^{t-\delta} \int_{\partial \Omega_{s}^{\epsilon}} \frac{\partial \Gamma_{i k}}{\partial N_{s}^{\epsilon}} u^{k} \phi d \sigma_{s}^{\epsilon} d s
$$

As $\|A\|_{\text {comm }} \leq \beta$ and $\vec{u}\left(\epsilon_{0}+P_{\gamma \epsilon_{0}} A(y, s), y, s\right)=\overrightarrow{0}$ we have

$$
|\vec{u}|\left(\epsilon+P_{\gamma \epsilon} A(y, s), y, s\right) \leq c_{\beta}\left(\epsilon_{0}-\epsilon\right) \tilde{N}_{*}(|\nabla \vec{u}|)\left(\epsilon_{0}+P_{\gamma \epsilon_{0}} A(y, s), y, s\right) .
$$

Using this and dominated convergence we find that the term under consideration tends to zero as $\epsilon-\epsilon_{0}, \delta \rightarrow 0$. We have therefore proved that the absolute value of,

$$
\sum_{k=0}^{n-1} \tilde{I}_{\epsilon, \delta}^{k}:=\sum_{k=0}^{n-1} I_{\epsilon, \delta}^{k}-\sum_{k=0}^{n-1} \int_{-\infty}^{t-\delta} \int_{\partial \Omega_{s}^{\epsilon}}\left(\frac{\partial u^{k}}{\partial N_{s}^{\epsilon}}-p N_{s}^{\epsilon, k}\right) \phi \Gamma_{i k} d \sigma_{s}^{\epsilon} d s
$$

tends to zero if $r \rightarrow \infty$ and $\epsilon-\epsilon_{0}, \delta \rightarrow 0$ in an appropriate manner. Now,

$$
\left(\frac{\partial u^{k}}{\partial N_{s}^{\epsilon}}-p N_{s}^{\epsilon, k}\right) \phi=\frac{\partial w^{k}}{\partial N_{s}^{\epsilon}}-p N_{s}^{\epsilon, k} \phi-u^{k} \frac{\partial \phi}{\partial N_{s}^{\epsilon}} .
$$

Arguing as above the integral over the third term in this expression will disappear as we pass to the limit with respect to $r$.

Let $F^{k}(Y, s)=w^{k} \Gamma_{i k}$ and $\Theta_{\epsilon}(\lambda, y, s)=\left(\lambda+\epsilon+P_{\gamma \epsilon} A(y, s), y, s\right)$. Then,

$$
\begin{aligned}
I_{\epsilon, \delta}^{k} & :=\int_{-\infty}^{t-\delta} \int_{\Omega_{s}^{\epsilon}} F_{s}^{k} d Y d s=\int_{-\infty}^{t-\delta} \int_{\mathbb{R}^{n-1}} \int_{0}^{\infty} F_{s}^{k} \circ \Theta_{\epsilon} d \lambda d y d s \\
& =\int_{-\infty}^{t-\delta} \int_{\mathbb{R}^{n-1}}^{\infty} \int_{0}^{\infty}\left(\left(F^{k} \circ \Theta_{\epsilon}\right)_{s}-\left(F^{k} \circ \Theta_{\epsilon}\right)_{\lambda} \frac{\partial P_{\gamma \epsilon} A}{\partial s}\right) d \lambda d y d s \\
& =\int_{\mathbb{R}^{n-1}}^{\infty} \int_{0}^{\infty} F^{k} \circ \Theta_{\epsilon}(\lambda, y, t-\delta) d \lambda d y
\end{aligned}
$$




$$
\begin{aligned}
& +\int_{-\infty}^{t-\delta} \int_{\mathbb{R}^{n-1}} F^{k} \circ \Theta_{\epsilon}(0, y, t-s) \frac{\partial P_{\gamma \epsilon} A}{\partial s} d y d s \\
& :=I_{1}+I_{2} \text {. }
\end{aligned}
$$

By standard arguments we get $\sum_{k} I_{1}^{k} \rightarrow u^{i}(X, t)$ as $\delta \rightarrow 0$. Using dominated convergence and Lemma 1.1 we have

$$
\left|I_{2}\right| \leq c \epsilon \int_{-\infty}^{t-\delta} \int_{\partial \Omega_{s}^{\epsilon}} \tilde{N}_{*}(|\nabla \vec{u}|)|\phi| \frac{\partial P_{\gamma \epsilon} A}{\partial s} d \sigma_{s}^{\epsilon} d s \rightarrow 0
$$

as $\epsilon-\epsilon_{0}$ and $\delta$ tends to 0 . Letting $r \rightarrow \infty$ and $\epsilon-\epsilon_{0}, \delta \rightarrow 0$ in an appropriate manner we may therefore conclude that

$$
\vec{u}(x, t)=S\left(\frac{\partial \vec{u}}{\partial \nu}\right)(x, t) .
$$

Using inequality (2) of Section 9 we may therefore conclude that $\frac{\partial \vec{u}}{\partial \nu}=\overrightarrow{0}$ a.e on $\partial \Omega^{\epsilon_{0}}$. This completes the proof of uniqueness in the regularity problem.

Uniqueness in the Neumann type problem. We assume that $\vec{u}$ solves the linearized system of Navier-Stokes in $\Omega$ with associated pressure $p$ and that $\frac{\partial \vec{u}}{\partial \nu}=\overrightarrow{0}$ a.e on $\partial \Omega$ in the sense of non tangential convergence. We furthermore assume that $\tilde{N}_{*}(|\nabla \vec{u}|)$, $\tilde{N}_{*, e}(p) \in L^{2}(\partial \Omega)$. We want to prove that $\vec{u} \equiv \overrightarrow{0}$ if $\vec{u}(X, t)=0$ for some point $(X, t) \in \Omega$. Following the argument used in [19] we will prove that

$$
\|\vec{u}\|_{R\left(\partial \Omega^{\epsilon}\right)}<\infty
$$

where $\Omega^{\epsilon}$ is defined as above and $\epsilon>0$. Assuming this we may use the uniqueness in the regularity problem to conclude that $\vec{u}=S \vec{q}_{\epsilon}$ for some $\vec{q}_{\epsilon} \in L^{2}\left(\partial \Omega^{\epsilon}\right)$. Using inequality (1) of Section 9,

$$
\left\|\vec{q}_{\epsilon}\right\|_{L^{2}\left(\partial \Omega^{\epsilon}\right)} \leq c\left\|\frac{\partial \vec{u}}{\partial \nu}\right\|_{L^{2}\left(\partial \Omega^{\epsilon}\right)} \rightarrow 0
$$

by dominated convergence as $\epsilon \rightarrow 0$. It therefore follows that $\vec{u} \equiv \overrightarrow{0}$ in $\Omega$ if $\vec{u}(X, t)=0$ for some point $(X, t) \in \Omega$ and the proof is finished.

We therefore have to prove $(*)$. Using Lemma 7.2 we may conclude that

$$
\int_{-\infty}^{\infty}\left\|\left\langle\frac{\partial \vec{u}}{\partial t}, N_{t}^{\epsilon}\right\rangle\right\|_{\left[L_{1}^{2}\left(\partial \Omega_{t}^{\epsilon}\right)\right]^{*}} d t \leq C\left(\left\|\tilde{N}_{*}(|\nabla \vec{u}|)\right\|+\left\|\tilde{N}_{*, e}(p)\right\|\right)^{2},
$$

and in order to prove $(*)$ all we have to prove is that

$$
\|\vec{u}\|_{L_{1,1 / 2}^{2}\left(\partial \Omega^{\epsilon}\right)}<\infty
$$

Define $\rho_{\epsilon}: \mathbb{R}_{+} \times \mathbb{R}^{n} \rightarrow \Omega^{\epsilon}$ in the following way:

$$
\rho_{\epsilon}(\lambda, x, t)=\left(\lambda+\epsilon+P_{\gamma(\lambda+\epsilon)} A(x, t), x, t\right)=\left(x_{0}, x, t\right) .
$$

For fixed $\epsilon$ and $\tilde{a}$ we put $\vec{w}=\vec{u} \circ \rho_{\epsilon}, \tilde{p}=p \circ \rho_{\epsilon}$ and let $N_{*}(\vec{w})(x, t)$ be the non tangential maximal function of $\vec{w}$ defined relative to $\Gamma_{\tilde{a}}(x, t)$ where $\tilde{a}$ is chosen so small that $N_{*}(\vec{w}) \leq \tilde{N}_{*}^{\epsilon}(\vec{u})$ at points corresponding under the transformation $(x, t) \rightarrow \rho_{\epsilon}(0, x, t)$. 
$N_{*, e}$ is similarly adjusted to the situation at hand. By the definition of the space $L_{1,1 / 2}^{2}\left(\partial \Omega^{\epsilon}\right)$ and since $\tilde{N}_{*}^{\epsilon}(|\nabla \vec{u}|) \in L^{2}\left(\partial \Omega^{\epsilon}\right)$ all we have to prove, in order to prove (**) is to prove that the restriction of $D_{1 / 2}^{t} \vec{w}$ to $\mathbb{R}^{n}$ has finite $L^{2}$-norm. We now intend to demonstrate the latter.

For a given $R>0$ we set

$$
\begin{gathered}
Q_{R}:=\left\{(\lambda, x, t) \in \mathbb{R}_{+} \times \mathbb{R}^{n}, \lambda<2 R,(x, t) \in B_{2 R}(0,0)\right\}, \\
m_{Q_{R}} \vec{w}:=\frac{1}{\left|Q_{R}\right|} \int_{Q_{R}} \vec{w}(x, t) d X d t .
\end{gathered}
$$

Adjusting the proof of Lemma 6.12 in [19], using the appropriate interior regularity result stated in Lemma 2.3, one may prove the following.

LEMma 10.2 .

$$
R^{-3} \int_{Q_{R}}\left|\vec{w}-m_{Q_{R}} \vec{w}\right|^{2} d X d t \leq c_{\beta}\left(\left\|N_{*}(|\nabla \vec{w}|)\right\|_{2}^{2}+\left\|N_{*, e}(\tilde{p})\right\|_{2}^{2}\right) .
$$

Let $\varphi \in C_{0}^{\infty}\left((-2 R, 2 R) \times B_{2 R}(0,0)\right)$ with $\varphi \equiv 1$ in $(-R, R) \times B_{R}(0,0)$ and

$$
\left\|\varphi_{t}\right\|_{\infty}+\left\|\nabla^{2} \varphi\right\|_{\infty} \leq c R^{-2}
$$

where

$$
\left|\nabla^{2} \varphi\right|^{2}:=\sum_{i, j=1}^{n-1} \varphi_{x_{i} x_{j}}^{2}+\sum_{i=1}^{n-1} \varphi_{x_{i} \lambda}^{2}+\varphi_{\lambda \lambda}^{2} .
$$

We will need the following lemma,

Lemma 10.3. Let $\vec{k}:=\left(\vec{w}-m_{Q_{R}} \vec{w}\right) \varphi$. If $z=(x, t)$ then,

$$
\int_{0}^{\infty} \int_{\mathbb{R}^{n}}\left|\nabla^{2} \vec{k}\right|^{2} \lambda d z d \lambda \leq c_{\beta}\left(\left\|\tilde{N}_{*}^{\epsilon}(|\nabla \vec{u}|)\right\|_{L^{2}\left(\partial \Omega^{\epsilon}\right)}^{2}+\left\|\tilde{N}_{*, e}^{\epsilon}(p)\right\|_{L^{2}\left(\partial \Omega^{\epsilon}\right)}^{2}\right),
$$

(ii) $\int_{0}^{\infty} \int_{\mathbb{R}^{n}}\left|\vec{k}_{t}\right|^{2} \lambda d z d \lambda \leq c_{\beta}\left(\left\|\tilde{N}_{*}^{\epsilon}(|\nabla \vec{u}|)\right\|_{L^{2}\left(\partial \Omega^{\epsilon}\right)}^{2}+\left\|\tilde{N}_{*, e}^{\epsilon}(p)\right\|_{L^{2}\left(\partial \Omega^{\epsilon}\right)}^{2}\right)$,

(iii) $\int_{0}^{\infty} \int_{\mathbb{R}^{n}}\left|\nabla D_{1 / 2}^{t} \vec{k}\right|^{2} \lambda d z d \lambda C_{\beta}\left(\left\|\tilde{N}_{*}^{\epsilon}(|\nabla \vec{u}|)\right\|_{L^{2}\left(\partial \Omega^{\epsilon}\right)}^{2}+\left\|\tilde{N}_{*, e}^{\epsilon}(p)\right\|_{L^{2}\left(\partial \Omega^{\epsilon}\right)}^{2}\right)$.

Proof of Lemma 10.3. We first consider (i). Let $\vec{k}:=\left(\vec{w}-m_{Q_{R}} \vec{w}\right) \varphi, \vec{k}=$ $\left(k^{0}, \ldots, k^{n-1}\right)$. For each $i \in\{0, \ldots, n-1\}$ recall that $w^{i}=u^{i} \circ \rho_{\epsilon}$. At the point $(\lambda, x, t)$ we have

$\left|\nabla^{2} k^{i}\right|^{2} \lesssim\left|\nabla^{2} u^{i}\right|^{2} \circ \rho_{\epsilon}+\left(u_{x_{0}}^{i} \circ \rho_{\epsilon}\right)^{2}\left|\nabla^{2} P_{\gamma(\lambda+\epsilon)} A\right|^{2}+c\left|\nabla w^{i}\right|^{2}|\nabla \varphi|^{2}+c\left|w^{i}-m_{Q_{R}} w^{i}\right|^{2}\left|\nabla^{2} \varphi\right|^{2}$

where we also have used Lemma 1.1. In order to prove the main estimates we need to prove one auxillary estimate. As $\tilde{N}_{*}^{\epsilon}(|\nabla \vec{u}|) \in L^{2}\left(\partial \Omega^{\epsilon}\right)$ we may for $i, j \in\{0, \ldots, n-1\}$ represent $\partial u^{i} / \partial x_{j}$ as a double layer potential $D \vec{f}=D_{\epsilon} \vec{f}_{\epsilon}$ with $\vec{f}=\vec{f}_{\epsilon} \in L^{2}\left(\partial \Omega^{\epsilon}\right)$. We 
may assume that $\vec{f}$ and $\partial u^{i} / \partial x_{j}$ have comparable $L^{2}\left(\partial \Omega^{\epsilon}\right)$-norms. This conclusion follows from the existence results of Section 9. Using Corollary 3.2 we therefore have

$$
\int_{0}^{\infty} \int_{\mathbb{R}^{n}}\left|u_{x_{j} x_{k}}^{i} \circ \rho_{\epsilon}(\lambda, z)\right|^{2} \lambda d z d \lambda \leq c\left\|\tilde{N}_{*}^{\epsilon}(|\nabla \vec{u}|)\right\|_{L^{2}\left(\partial \Omega^{\epsilon}\right)}^{2} .
$$

Using this estimate and familar arguments one may therefore easily prove that

$$
\begin{gathered}
\int_{0}^{\infty} \int_{\mathbb{R}^{n}}\left|\nabla^{2} k^{i}\right|^{2} \lambda d z d \lambda \leq C\left[\left\|\tilde{N}_{*}^{\epsilon}(|\nabla \vec{u}|)\right\|_{L^{2}\left(\partial \Omega^{\epsilon}\right)}\right. \\
\left.+\int_{0}^{\infty} \int_{\mathbb{R}^{n}}\left(\left|\nabla w^{i}\right|^{2}|\nabla \varphi|^{2}+\left|w^{i}-m_{Q_{R}} w^{i}\right|^{2}\left|\nabla^{2} \varphi\right|^{2}\right) \lambda d z d \lambda\right] .
\end{gathered}
$$

But

$$
\int_{0}^{\infty} \int_{\mathbb{R}^{n}}\left|\nabla w^{i}\right|^{2}|\nabla \varphi|^{2} \lambda d z d \lambda \leq c\left\|\tilde{N}_{*}^{\epsilon}(|\nabla \vec{u}|)\right\|_{L^{2}\left(\partial \Omega^{\epsilon}\right)}^{2},
$$

by familar arguments. As $\varphi \equiv 0$ on the complement of $Q_{R}$ we may use the inequality stated in Lemma 10.2 to conclude that

$$
\begin{aligned}
\int_{0}^{\infty} \int_{\mathbb{R}^{n}}\left|w^{i}-m_{Q_{R}} w^{i}\right|^{2}\left|\nabla^{2} \varphi\right|^{2} \lambda d z d \lambda & \leq c\left(\left\|N_{*}(|\nabla \vec{w}|)\right\|_{L^{2}\left(\partial \Omega^{\epsilon}\right)}^{2}+\left\|N_{*, e}(\tilde{p})\right\|_{L^{2}\left(\partial \Omega^{\epsilon}\right)}^{2}\right) \\
& \leq c_{\beta}\left(\left\|\tilde{N}_{*}^{\epsilon}(|\nabla \vec{u}|)\right\|_{L^{2}\left(\partial \Omega^{\epsilon}\right)}^{2}+\left\|\tilde{N}_{*, e}^{\epsilon}(p)\right\|_{L^{2}\left(\partial \Omega^{\epsilon}\right)}^{2}\right) .
\end{aligned}
$$

We now treat part (ii) of the lemma. At the point $(\lambda, x, t)$ we have

$$
\left|k_{t}^{i}\right|^{2} \lesssim\left|u_{t}^{i}\right|^{2} \circ \rho_{\epsilon}+\left(u_{x_{0}}^{i} \circ \rho_{\epsilon}\right)^{2}\left|\frac{\partial P_{\gamma(\lambda+\epsilon)} A}{\partial t}\right|^{2}+\left|w^{i}-m_{Q_{R}} w^{i}\right|^{2}\left|\varphi_{t}\right|^{2} .
$$

As

$$
\begin{aligned}
\int_{0}^{\infty} \int_{\mathbb{R}^{n}}\left|u_{t}^{i} \circ \rho_{\epsilon}\right|^{2} \lambda d z d \lambda & \leq \int_{0}^{\infty} \int_{\mathbb{R}^{n}}\left|\Delta u^{i} \circ \rho_{\epsilon}\right|^{2} \lambda d z d \lambda+\int_{0}^{\infty} \int_{\mathbb{R}^{n}}\left|\frac{\partial p}{\partial x_{i}} \circ \rho_{\epsilon}\right|^{2} \lambda d z d \lambda \\
& \leq c_{\beta}\left(\left\|\tilde{N}_{*}^{\epsilon}(|\nabla \vec{u}|)\right\|_{L^{2}\left(\partial \Omega^{\epsilon}\right)}^{2}+\left\|\tilde{N}_{*, e}^{\epsilon}(p)\right\|_{L^{2}\left(\partial \Omega^{\epsilon}\right)}^{2}\right),
\end{aligned}
$$

by case (i) and elliptic theory, case (ii) can be handled by the same argument as in case (i).

To consider (iii) note that

$$
\begin{aligned}
\int_{0}^{\infty} \int_{\mathbb{R}^{n}}\left|\nabla D_{1 / 2}^{t} k^{i}\right|^{2} \lambda d z d \lambda & =\int_{0}^{\infty} \int_{\mathbb{R}^{n}}\left\langle\nabla D_{1 / 2}^{t} k_{\lambda}^{i}, \nabla D_{1 / 2}^{t} k^{i}\right\rangle \lambda^{2} d z d \lambda \\
& \leq\left(\int_{0}^{\infty} \int_{\mathbb{R}^{n}}\left|\nabla k_{\lambda}^{i}\right|^{2} \lambda d z d \lambda\right)^{1 / 2} \times\left(\int_{0}^{\infty} \int_{\mathbb{R}^{n}}\left|\nabla k_{t}^{i}\right|^{2} \lambda^{3} d z d \lambda\right)^{1 / 2} .
\end{aligned}
$$


The first factor in this product is handled using part (i) of the lemma. We are left with the second factor. But at the point $(\lambda, x, t)$ we have

$$
\begin{gathered}
\left|\nabla k_{t}^{i}\right|^{2} \lesssim\left|\nabla u_{t}^{i}\right|^{2} \circ \rho_{\epsilon}+\left|\nabla u_{x_{0}}^{i} \circ \rho_{\epsilon}\right|^{2}\left|\frac{\partial P_{\gamma(\lambda+\epsilon)} A}{\partial t}\right|^{2} \\
+\left|u_{x_{0}}^{i} \circ \rho_{\epsilon}\right|^{2}\left|\nabla \frac{\partial P_{\gamma(\lambda+\epsilon)} A}{\partial t}\right|^{2}+\left|\nabla w^{i}\right|^{2}\left|\varphi_{t}\right|^{2}+\left|w_{t}^{i}\right|^{2}|\nabla \varphi|^{2}\left|w^{i}-m_{Q_{R}} w^{i}\right|^{2}\left|\nabla \varphi_{t}\right|^{2} .
\end{gathered}
$$

Using part (i), (ii) of the lemma as well as the argument in the proof of part (i) and (ii) we have,

$$
\int_{0}^{\infty} \int_{\mathbb{R}^{n}}\left|\nabla k_{t}^{i}\right|^{2} \lambda^{3} d z d \lambda \lesssim \int_{0}^{\infty} \int_{\mathbb{R}^{n}}\left|\nabla u_{t}^{i}\right|^{2} \lambda^{3} d z d \lambda+\left\|\tilde{N}_{*}^{\epsilon}(|\nabla \vec{u}|)\right\|_{L^{2}\left(\partial \Omega^{\epsilon}\right)}^{2}+\left\|\tilde{N}_{*, e}^{\epsilon}(p)\right\|_{L^{2}\left(\partial \Omega^{\epsilon}\right)}^{2} .
$$

But,

$$
\int_{0}^{\infty} \int_{\mathbb{R}^{n}}\left|\nabla u_{t}^{i}\right|^{2} \lambda^{3} d z d \lambda \leq C_{\beta}\left(\left\|\tilde{N}_{*}^{\epsilon}(|\nabla \vec{u}|)\right\|_{L^{2}\left(\partial \Omega^{\epsilon}\right)}^{2}+\left\|\tilde{N}_{*, e}^{\epsilon}(p)\right\|_{L^{2}\left(\partial \Omega^{\epsilon}\right)}^{2}\right)
$$

using interior regularity and elliptic estimates.

Using the same deductions as in $[19, p .403]$ one may prove that

$$
\begin{aligned}
& \left\|\left.D_{1 / 2}^{t} \vec{k}\right|_{\mathbb{R}^{n}}\right\|_{2}^{8} \\
\leq & c\left(\int_{0}^{\infty} \int_{\mathbb{R}^{n}}\left|\vec{k}_{\lambda \lambda}\right|^{2} \lambda d \lambda d z\right) \times\left(\int_{0}^{\infty} \int_{\mathbb{R}^{n}}\left|D_{1 / 2}^{t} \vec{k}_{\lambda}\right|^{2} \lambda d \lambda d z\right)^{2} \times\left(\int_{0}^{\infty} \int_{\mathbb{R}^{n}}\left|\vec{k}_{t}\right|^{2} \lambda d \lambda d z\right) .
\end{aligned}
$$

Plugging the estimates of Lemma 10.3 into this inequality we may conclude that,

$$
\left\|\left.D_{1 / 2}^{t} \vec{k}\right|_{\mathbb{R}^{n}}\right\|_{2} \leq C\left(\left\|\tilde{N}_{*}^{\epsilon}(|\nabla \vec{u}|)\right\|_{L^{2}\left(\partial \Omega^{\epsilon}\right)}+\left\|\tilde{N}_{*, e}^{\epsilon}(p)\right\|_{L^{2}\left(\partial \Omega^{\epsilon}\right)}\right)<\infty .
$$

To prove the same statement for $\vec{w}$ one proceeds as follows. Note that

$$
\left\|\left.D_{1 / 2}^{t} \vec{w}\right|_{\mathbb{R}^{n}}\right\|_{2}^{2} \sim \int_{\mathbb{R}} \int_{\mathbb{R}} \int_{\mathbb{R}^{n-1}} \frac{(\vec{w}(x, s)-\vec{w}(x, t))^{2}}{|s-t|^{2}} d x d s d t .
$$

But as, by definition, $\vec{k}=\vec{w}-\vec{c}$ on $(-R, R) \times B_{R}(0,0)$ for some constant $\vec{c}$,

$$
\begin{aligned}
& \int_{-R}^{R} \int_{-R}^{R} \int_{\mathbb{R}^{n-1}} \frac{(\vec{w}(x, s)-\vec{w}(x, t))^{2}}{|s-t|^{2}} d x d s d t=\int_{-R}^{R} \int_{-R \mathbb{R}^{n-1}}^{R} \int_{\mathbb{R}_{\mathbb{R}}} \frac{(\vec{k}(x, s)-\vec{k}(x, t))^{2}}{|s-t|^{2}} d x d s d t \\
& \leq \int_{\mathbb{R}^{n-1}} \frac{(\vec{k}(x, s)-\vec{k}(x, t))^{2}}{|s-t|^{2}} d x d s d t \\
& \sim\left\|\left.D_{1 / 2}^{t} \vec{k}\right|_{\mathbb{R}^{n}}\right\|_{2}^{2} \leq C\left(\left\|\tilde{N}_{*}^{\epsilon}(|\nabla \vec{u}|)\right\|_{L^{2}\left(\partial \Omega^{\epsilon}\right)}+\left\|\tilde{N}_{*, e}^{\epsilon}(p)\right\|_{L^{2}\left(\partial \Omega^{\epsilon}\right)}\right)<\infty .
\end{aligned}
$$

Letting $R \rightarrow \infty$ we get the estimate we wanted. This completes the proof of uniqueness in the Neumann problem. 


\section{REFERENCES}

[1] R.M. Brown, The method of layer potentials for the heat equation in Lipschitz cylinders, Amer. J. Math., 111 (1989), pp. 359-379.

[2] R.M. Brown, The initial-Neumann problem for the heat equation in Lipschitz cylinders, Trans. A.M.S., 320 (1990), pp. 1-52.

[3] R.M. Brown, P.Perry, Z. Shen, On the dimension of the attractor for the non-homogeneous Navier-Stokes equations in non-smooth domains, Indiana Univ. Math. J., 49, no. 1 (2000), pp. 81-112.

[4] R.R. Coifman, G. David, Y. Meyer, La solution des conjecture de Calderón (French) [Solution of Calderon's conjectures], Adv. in Math., 480, no. 2 (1983), pp. 144-148.

[5] R.R. Coifman, G. David, Y. Meyer, Lintégral de Cauchy définit un opérateaur borné sur $L_{2}$ pour les courbes lipschitziennes, Annals of Math., 116 (1982), pp. 361-387.

[6] P. Constantin, C. Foias, Navier-Stokes Equations, The University of Chicago press (1988).

[7] B.E.J. Dahlberg, Poisson semigroups and singular integrals, Proc. Amer. Math. Soc., 97, no. 1 (1986), pp. 41-48.

[8] B.E.J. DAHLBERG, Weighted norm inequalities for the Lusin area integral and the nontangential maximal functions for functions harmonic in a Lipschitz domain, Studia Math., 67 no. 3 (1980), pp. 297-314.

[9] B.E.J. Dahlberg, C.E. Kenig, J. Pipher, G.C. Verchota, Area integral estimates for higher order elliptic equations and systems, Annales de l'Inst. Fourier, 47 (1998), pp. 1425-1461.

[10] B.E.J Dahlberg, C.E Kenig, G.C Verchota, Boundary value problems for the systems of elastostatics in Lipschitz domains, Duke. Math. J., 57 (1988), pp. 795-818.

[11] P. Deuring, Paul, W. von Wahl, Strong solutions of the Navier-Stokes system in Lipschitz bounded domains, Math. Nachr, 171 (1995), pp. 111-148.

[12] E.B. Fabes, J.E. Lewis, N.M.Riviere, Boundary value problems for the Navier-Stokes equations, Amer. J. of Math., 99 (1977), pp. 626-668.

[13] E.B. Fabes, M. Jodeit, $L^{p}$ boundary value problems for parabolic equations, Bull. A.M.S, 74 (1968), pp. 1098-1102.

[14] E.B. Fabes, C.E Kenig, G.C Verchota, The Dirichlet problem for the Stokes system on Lipschitz domains, Duke. Math. J., 57 (1988), pp. 769-793.

[15] E.B. Fabes, N.M.Riviere, Symbolic calculus of kernels with mixed homogeneity, in Singular Integrals, A.P. Calderon, Ed., Proc. Symp. Pure. Math., A.M.S., Providence, 10 (1967), pp. $106-127$.

[16] E.B. FABes, N.M.Riviere, Singular integrals with mixed homogeneity, Studia Math., 27 (1966), pp. 19-38.

[17] W. GAO, Layer potentials and boundary value problems for elliptic systems in Lipschitz domains, J. Func. Anal., 95 (1991), pp. 377-399.

[18] S. Hofmann, Parabolic singular integrals of Calderon-type, rough operators and caloric layer potentials, Duke Math. J., 90 (2) ((1997)), pp. 209-259.

[19] S. Hofmann, J.L Lewis, $L^{2}$ solvability and representation by caloric layer potentials in timevarying domains, Annals of Math, 144 (2) (1996), pp. 349-420.

[20] S. Hofmann, J.L Lewis, The $L^{p}$-regularity problem for the heat equation in non-cylindrical domains, preprint.

[21] S. Hofmann, J.L Lewis, The $L^{p}$-Neumann problem for the heat equation in non-cylindrical domains, preprint.

[22] R. Kauffman, J.M Wu, Parabolic measure on domains of class Lip ${ }_{1 / 2}$, Comp. Math., 65 (1988), pp. 201-207.

[23] C.E. Kenig, Elliptic boundary value problems in Lipschitz domains, In Beijing Lectures in Harmonic Analysis, E.M.Stein, ed., Ann. of Math. Studies, 112 (1986), pp. 131-183.

[24] C.E. Kenig, Harmonic Analysis Techniques for Second Order Elliptic Boundary Value Problems, CBMS series in Mathematics, no. 83.

[25] J.L. Lewis,M. Murray, The method of Layer potentials for the heat equation in time-varying domains, Memoir of the A.M.S, no. 545.

[26] J.L. Lewis, J. Silver, Parabolic measure and the Dirichlet problem for the heat equation in two dimensions, Indiana U. Math. J., 37 (1988), pp. 801-839.

[27] K. Nyström, Boundary value problems for parabolic Lamé systems in time-varying domains, 
Research report, Department of Mathematics, Umeå University and to appear in Indiana University Math. Journal, 18 (1998).

[28] E.M. Stein, Singular integrals and differentiability properties of functions, Princeton Univ. Press (1970).

[29] E.M. Stein, Harmonic Analysis, Princeton. Univ. Press (1993).

[30] G.C. Verchota, Layer potentials and regularity for the Dirichlet problem for the Laplace's equation in Lipschitz domains, J. of Func. Anal., 59 (1984), pp. 572-611.

[31] Z. Shen, A note on the Dirichlet problem for the Stokes system in Lipschitz domains, Proc. Amer. Math. Soc., 123, no. 3 (1995), pp. 801-811.

[32] Z. SHEN, Boundary value problems for parabolic Lame systems and a nonstationary linearized system of Navier-Stokes equations in Lipschitz cylinders, Amer. J. of Math., 113 (2) (1991), pp. 293-374. 